

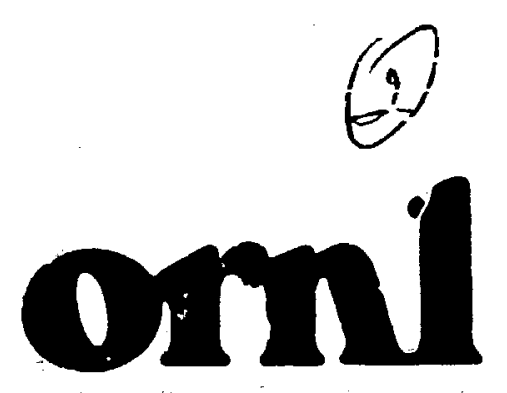

OAK

RIDGE

NATIONAL

LABORATORY
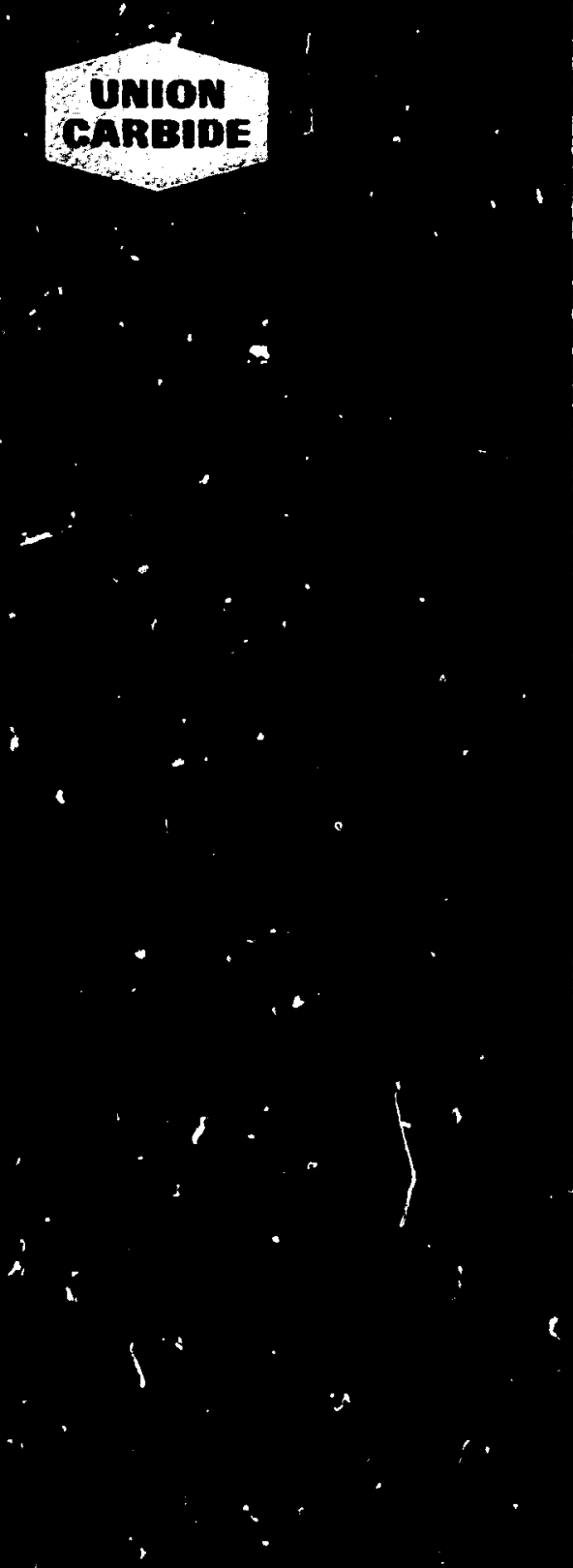

IJPERATEU BY

- HNion cargade corpopation FDH THF UNITED SIATES ULPARIMFNT OF FNFHCYY

$$
2-1^{89}
$$

\section{MASTER}

\section{A Users Manual for the FORSS Sensitivity and Uncertainty Analysis Code System}
J. L. Lucius
C. R. Weisbin
J. H. Marable
J.D. Drischler
R. Q. Wright
J.E. White 
ORNL-5316

Distribution rategory UC-79d

(ENDF-291)

Contract No. H-7405-eng-26

- Computer Sciences division and

Engineering Physics Division

A USERS MANUAL FOR THE FORSS SENSITIVITY

AND UNCERTAINTY ANALYSIS CODE SYSTEM
J. L. Lucius ${ }^{\star}$,
C. R. Heisbint+
J. H. Marable +
J. D. Drischler
R. Q. Wright ${ }^{\star}$
J. E. White ${ }^{\star}$

\footnotetext{
* Computer Sciencrs Divisior,

tEngineering Physics Division
}

Date Published: January 1981
OAK RIIGE NATIONAL LABORATORY
Oak Ridge, Tennessee 37830
operated by
UNION CARBIDE CORPURATION
for the
U. S. Department of Energy


Chapter No.

Page No.

ABSTRACT ...................

ACKNOWLEDGEMENTS .................... vi

I. IRTRODUCTION .................. I-I

A. Cross Seccion Processing ............ I-1

B. ENDF/B Uncertzinty File Processing ........ I-2

C. Neutronics Calculations ............ I-2

D. The JULIET Mocinle ............... I-2

E. Two-Dimensionai Analysis With DOT or

VENTURE Neutronics.............. I

F. The SENPRO Service Mudule .......... I-3

G. Cross Section Difference Effects ........ I-3

H. COVERT ....................... I-4

I. The COVERX Service Module .......... I-4

J. Uncertainties Due Exclisively to Nciclear Data Uncertainties............ . $1-4$

K. Uncertainties With Integral Experiments Taken Into Account ............. I-4

L. COVERS ...................... I-5

M. ADJUST ...................... I.5

N. The Inverse Problem ............ I -5

0. System Structure............... . I-5

P. FORSS Input Preparation ............ . I.

11. THE IMPROVE.T FIDO INPUT SYSTEM . . . . . . . . . . II-I

III. THE ANISN, AMPX, ISOTOX TC MATXS TRANSLATORS . . . . . III-I 
Chapter No.

Page No.

IV. THE FORSS DATA REPOSITORY ............. IV I

V. INPUT INSTRUCTIONS FOR ANISN AND I.ANIIX ........ V-1

VI. INPUT INSTRUCTIONS FOR GENERALIZED CALCULATIONS

HITH FORSS ANISN .................. VI-1

vII. A USER'S GUIDE FOR THE JULIET MUDULE OF THE

FORSS SYSTEM .................. VII-I

VIII. THE SENPRO SERVICE MODULE OF THE FORSS SYSTEM . . . . VIII-I

IX. SENDIN AND SENTINEL: THO COMPUTER CODES TO

ASSESS THE EFFECTS OF NUCLEAR DATA CHANGES ..... . IX-1

$X$. COVERT AND CAVALIER: THO COMPUTER CODES FOR

ESTIMATING UNCERTAINTIES OF CALCULATED NEUTRONICS

PARAMETERS USING STANDARD INTERFACE FILES SENPRO

AND COVERX ................... $\mathrm{x}-1$

XI. THE COVERX SERVICE MODULE OF THE FORSS SYSTEM ..... XI-1

XII. THE IJRCOVER MOOULE OF THE FORSS SYSTEM. ........ XII-I

XIII. THE COVERS MODULE OF THE FORSS SYSTEM ......... XIII-1

XIV. THE ADJUST MOOULE OF THE FORSS SYSTEM . ....... XIV-1

XV. THE NUTCRACKER MODULE GF THE FORSS SYSTEM ....... XV-1 
ABSTRACT

FORSS is a code system used to studj relationships between nuclear reaction cross sections, integral experiments, reactor performance parameter predictions and associated uncertainties. This report describes the computing environment and the modules currently used to implement ForSS Sensitivity and Uncertainty Methodology. 


\section{ACKHOWLEDGMENTS}

The authors gratef:Illy acknowledge the significant contributions of the following people to the development of the FOnSS system:

- E. M. Oblow for guidarce throughout the design and development of FORSS,

- N. M. Greene, L. M. Petrie, and H. E. Ford, III for consultation on the development of driver-controlled, modular code systerrs, ard cross section processing methods,

- D. E. Bartine and J. V. Pace for detailed descriptions of tha SHANLAKE algorithms,

- J. J. Magschal for many informative discussions and reviews of cross section adjustment procedsres,

- R. L. Childs for consultation on implementing two-dimensional sensitivity analysis,

- R. A. Anderl, B. L. Broadhead, R. L. Childs, J. M. Ryskamp, Y. Seki, C. 0. Slater, J. J. Hagschal, C. C. Hebster, and Y. Yeivin for using the system aril contributing to its validation,

- R. W. Roussin for many helpful suggestions on the decumentation and distribution of the system,

- J. Ching for implementing a version of the system to execute on control data computers,

- M. B. Enmett for assistance in implemen:ing the Intrigue graphics display package,

- W. E. Ford, III and R. W. Roussin for reviewing this manuscript. 
vij

The combined efforts of the administrative support staffs of both the Eng', leering Physics Division and the Computer Sciences Division are sincereiy appreciated. The production of this repsrt through numerous drafts and revisions over a period of six years was accomplished by:

Barbara Beem

Patty Boit

Thelma Braidsford

Billie Ferguson

Virgiria Gi idewell

Jeanette Hamby

Si:ndi Henr:'

Ann Houston

Katie Lawhorn

Mancy Mauney

Brenda Neeley

Cathy 01dhan

Susan Rióer

Coralee Zeigler

The development of the FORSS s.rstem was made possible by the support and encouragement of F. C. Maienschein, F. R. Mynatt, and 6. E. Whitesides. 
CHAPTER !

Table of Contents

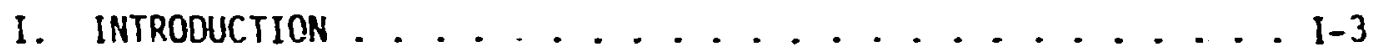

A. Cross Section Processing ............ I-3

8. ENDF/B Uncertainty File Proce ssing ......... I-4

C. Neutronics Calculations ............ I-4

. The Jül IE Module . . . . . . . . . . 1.4

E. Two Dimensic...s Analys is with DOT or VENTUPE

Neutronics ............... 1-5

F. The SENPRO Service Moduie .......... 1-5

G. Cross Section Difference Fffects ......... I-5

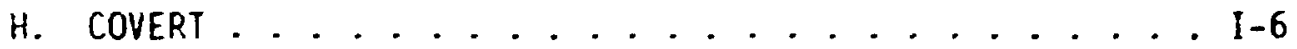

I. The CJVERX Selice Module ............. I-6

j. Urcertainties Due Exclusively tc Nuclear Data Uncertainties ...................

‥ Uncertainties with Integral Experiments taken into Account ............... I-6

L. COVERS ..................... I-7

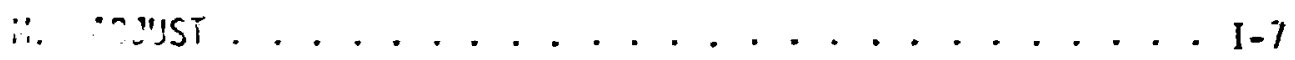

N. The Inverse Pronlem ............ I-7

0. System Structurs ............... I-7

P. FORSj Input Preparation .............. I-9

11. REFERENCES ....................... . I-12 


\section{INTROQCTION}

The purpose of this document is to provide guidance on the use of the computational methods that are germane to FORSS I Sensitivity and Uncer'ainty Methodolngy. In its totality FORSS is not a single code system but rather a collection of code systems and stand alone codes that contribute to the s)lution of a FORSS problem. FORSS includes multigroup cross section procissing, uncertainty file processing, neulronics calculations, source calculations. sensitivity profile generation, uncertainty analysis, and estimation of accuracy, requirements for nuclear data to meet pre-detemined design constraints and error margins. A modular code system under driver control has been develisped and is commonly called FuRSS; however, it does not at present place all the FORSS methodology under tre control of a single driver. This docunent will attempt to show the relationship cf all the codes and code systems that contribute to the solution of a FORSS problem. Some of the major code sustems lised are weli established and documented. Such documentation will not be duplicated here but:;ill be referencod as required. The scope of this report assumes familiarity with the discussion of FORSS methodology found in reference 1.

\section{A. Cross Section Frocesining}

Multigroup cross sections must be prepared for the neutronics calculation selected to model the assembly being investigated. The MINX-SPHINX-, and the AMPX ${ }^{4}$ cross section processing systems are examples of cross section processors that provide this capability. The AXMIX code is also useful in cross secticn preparation. However, in sensitivity and uncertainty analysis partial cross sections must be available. FORSS uses the proposed $\operatorname{cCCC}^{5}$ file MATXS 7 as the data base for partial cross sections. The aforementioned cross section processors do not now produce a MATXS file al though such capability is currently being tested. At present, the FOR:S system includes three translators for producing MATXS from the more mature ISOTXSE AMPX master, or ANISNE formats. 
B. ENDF/B Uncertainty File Processing

Uncertainty analysis withir the FORSS systen requires that multigroup covariance matrices be produced from ENDF/B ${ }^{9}$ formatted uncertainty files. Covariance matrices are produced by the PUFFlo code. The matrices are placed in a proposed CCCC file COVERX ${ }^{11}$. COVERX files must be available before uncertainty analysis can be initiated. If appropriate, available multigroup covariance matrices can be used directly thus bypassing the PUFF processing step.

\section{Neutronics Calculations}

The ANISN, DOT 12 , and VERTUPE ${ }^{13}$ neutronics codes are used in the FORSS methodology. ANISN is a module in the FORSS driver controlled system and its interaction with the other modules in the system is somewhat automated. Comments about the use of DOT and VENTURE in FCRSS will be made in Section $E$.

The number of neutronics calculations required for a study is a function of the number and type of responses under consideration. Sensitivity analysis for criticality requires a forward and adjoint neutronics calculation; reaction rate ratio analysis requires, in addition, the computation of a generalized adjoint. Few worth sensitivity, four neutronics calculations are required; these being a forward and adjoint, and a generalized forward and adjoint. The JULiET module uses tine fluxes produced by these neutronics calculations.

\section{The JULIET Mordule}

This moduie calculates sources, responses, normalization parameters and sensitivity coefficients. The JULIE? algorithms for generalized souries, responsas, and normzlization parameters use FORSS ANISN (FANISN) produced angular fluxes. The sources calculated by JULIET are returned to FANISH as input to generalized neutronics calculations. A more detailed description of the FANISN and JULIFT data interchange mecnanism is available to Chapter IV.

The sensitivity coefficients calculated by JULIET are placed in a proposed CCCC fiie SËNPROIl. 
JULIET is organized into =xecution paths, i.e., it has miltiple entry points. A source calculation may be performed and execution terminated without generating sensitivity coefficients. When sufficient results are available from prel iminary calculations, JULIET may be asked to generate sensitivity coefficient.s and bypass the source calculation.

\section{E. Two-Dimensional Ana?ys is With DOT or VENTURE Meutronics}

With an understanding of how ANISN and JLIET inceract to produce generalized t7uxes, sources, and nomalization parameters, the use of 2-D neutronics in FORSS can be clarified. Before sensitivity coefficients are generated, JULIET prepares a so-called $\langle\theta \star \theta\rangle$ file. This is symbolic sirce it could be $\langle\Gamma *\rangle$ or $\langle\theta * \Gamma\rangle$ (brackets inply integration over spatial regions and angles). This file is used in the generacion of sensitivity cuefficients. then 2-D neutrunics is used in FORSS, the selected 2-D code system, DOT or VENTURE, is used to produce the equiva!ent fiuxes as produced by ANISN arid the JULIET-SOURCE algorithms. The VIPI4 code is then used to prepare tree $\langle\emptyset \star \theta\rangle$ file. The selected $2-D$ code system must also produce the normalization parameters required by the JULIET sensitivity profile calculation. With appropriate $\langle\theta * \phi\rangle$ files and nomalization parameters available, the JULIET-SENSE execution path is entered and the problem solution path is tine same as when ANISN neutronics is used.

\section{F. The SENPRO Service Module! $\equiv$}

JULIET generates the SENPRO file containing sensitivity profiles. However, before using the file in uncertainty analysis, it may be desirable to examine the file(s) in detail. The SENPRO service module operates on the file proviaing a number of services including plotting and listing. A complate inventory of the modules capabilities is available in Chapter VIII.

\section{G. Cross Section Difference Effects}

When the calculation of sensitivity coefficients is complete and SENPRO file(s) are available, the effects of proposed cross section modifications may be investigated. The SENTINEL 16 module computes the perceritage 
(or fractional) change in the performance parameter of a given assembly due to specified percentage (or fracticnal) changes in desic... ' 20 reaction cross sertions over a number of energy rogions. An edited ist of the most significant individual contributions to the response char.ge is also provided.

\section{H. CONERT 17}

The modile COVERT copies a covariance file in COVÉRX format, and in the copying process changes the file representation from binary to card image furm or vice versa. In addition; a complete listing ór a partial listing of the file is printed to give the user infurmation about the contents of a particular file.

\section{The COVERX Service Modile}

As stated in Section B, COVERX files nust be avai?able before uncertaintv analysis can be initiated with the CAVALIER or UNCOVER modules.

The COVERX service module operates on the file providing a number of services such as i isting and merging. A coriplete irventory of the capabilities is available in Chapter XI.

J. Uncertainties Due Exclusively to Nuclear Data Uncertainties

When COVERX and SFNPRO files are available, uncertainty analysis may be initiated. The CFVALIER17 module folds sensitivity profiles (SENPRO) and covariance matrices (COVERX) to estimate reactor performance uncertainties wich result from uncertainties in nuclear data.

K. Uncertainties with Integral Experiments Taken Into Accoun:

The modvie UNCOVER ${ }^{18}$ finds the adjustments of both integral and differential data which are most consistent (in a general least squares sense) with the data and with the calculational model as represented by the sensitivity ccefticients. The input to INCOVER includas data from SENPRO 
sensitivity and COVERX (a priori) covariance files. The output includes a posteriori covarianc's for the adjusted data in addition to the adjustments themselves. The adjusted data and associated covariances can be applied to cesign models for improved predictions of performance parameter ano of their uticteránties.

\section{COVERS}

COVERS is a convenience module whose sole function is to process data from a SENFRO file and a COVERX file into informal files accepiable as input to the UNCOVER module.

\section{ADJUST}

The ADJUST module modifies the cross sections on an AMPX master file by the percent changes calculated by the UnCOVER module. This creates an adjusted cross section library for dissemination via the AMPX system.

N. The Inverse Problem

The NUTCRCKR " module is a first attempt at solution of the "Inverse Problem" of ieactor sensitivity. The objective is to provide quantitative guidance to data acquisition, data evaluation, and associated data processing code development programs by giving estimated accuracy requirements on various inu?tigroup nuclear data to meet predetermined design constraints and error margiris.

\section{Sy!, tem Structure}

The system is composed of modules which may be execsted under DRIVER cortmol or they may be executed as stand-alone programs. Under DRIVER control, a run is executed from a procedure which includes most of the IBM JCL needed. We are indebted to the AMPX project for the ORIVER and procedure technology. 
Cata interchange between modules is accomplished by well defined files which are iocated on external storage devices such aś magnetic tape or disks.: Proposed cccc files are used for partial c'jss sections (MATXS), sensitivity coeficicients (SENPRO), and covariance matrices (COVERX). In this version of the systen, FORSS ANISN uses a traditional ANISN formatted tape which is discussed in Chapter $v$. The COVERS module prepares from SENPRO and CrVIIPX files two other files which are suitably formatted for the flexible structurs of the UNCOVER module. FORSS ANISN and JULIET interchange, data via a repository mich is implemented with the FMANG nodule. FMAMG and the repository are discussed in Chapter IV.

FORSS is designed to permit multiple entry points into the system. A canprehensive calculation may be designed to generate fluxes, normalization parameters, sensitivity coefficients and perform an adjustment in a single-computer run. Conversely, many computer runs may te ased to obtain the same results. The latter appioach requires that the appropriate files (Repository, SENPRO, cOVERS files, etc.) must be saved cn semipermanent external storage devices. It is also good policy to do this for the comprehensive run u.iless the calculation is small enough to make little demand on computer resources. A correct calculational hierarchy ust be defined and executed for either the comprehensive or the segmented solution. A correct hierarchy is determined by the prerequisites of the modules needed to solve the problem of-interest. An example of a correct hierarchy is:

1. Processing of F.NDF/B files into multigroup neutron cross sections. Resulting files may be an AMPX master, ISOTXS, or ANISN crosssection library.

2. Processing of ENDF/B uncertainty files resulting in a COVERX file. NOTE: The FORSS DRIVER controlied program does not include the modules needed to implement sters 1 and 2; these are external preparatory steps for FORSS.

3. Generating forward and adjoint fluxes on the repository with FANISN.

4. Translating AMPX, ISOTXS, or ANISN cross sections to the MATXS file with the appropriate translation module. 
5. Generating normalization parameters and generalized sources on the repository with JULiET.

6. Generating generalized forward and adjoint fluxes on the repository with FANISN.

7. Calculating sensitivity coefficients and preparing a SENPRO file(s) with JULIET.

8. Operating en SEMPRO files with the SENPRO SERVICE moduie.

9. Performing perturbation onalysis with SENTINEL.

10. Operating on COVERX files with the COVERX SERUICE module.

11. Performing uncertainty andlysis with CAVALIER.

12. Preparing input for UNCOVER HITH COVERS.

13. Performing uncercainty analysis and adjustment with UNCOVER.

The mu?tiple entry feature of FORSS permits entry at the module level and in some situations a: the submodule level. Some modules perform more than one bask and some r.i these tasks may be selected for execution in a partirutar run and others excluded. This concept is referred to as execution paths.

FORSS has a very powerful ald flexible free field data input scheme called Improved FIDO which is discussed in Chapter I?.

\section{P. FORSS Input Preparation}

Detailed input requirements for each module are included in the chapters describing the moduies. The user creates an input stream by praparing = MOONAME cards and, where appropriate, execution path names. Inmediately following the = MODAAME and execution path names place the required iriput cards.

Example:

=FMANG - Repository manaser (input data, see Chupter IV).

=ITOM - ISOTXS to MRTXS translator (ro input data required;.

=ANTMX - ANISN to MATXS translator (input data, see Chapter III).

=AMPXM - AMPX to MATXS translator (input data, see Chapter III). 
=PNATXS

=FANISN

=FJULIE

MIX

FLUX

SOLRCE

SENEE

=SEIIPRO

LIST

PLOT

คอD

MERGE

SEND

SIZE

MORM

FIX

GRID
- Lists a MATXS file (no input data required).

- I-D $S_{n}$ transport calculation (input data, see Chapter V).

- iULIET for sources and sensitiviky coefficients

- execution path for operator definitir,il inpet data, see (napter VII).

- execition path for flux preparation and $\phi^{*} \phi$ generation. (Input datu, see Chapter VII).

- execution path for response and source calculation (input data, see Chapter viIi.

- execution path for gerieration of senstivity coefficients (Input vata, see Chapter VII!.

- Senpro service module.

- execution path of a file list (input data, see Chapter VIII).

- execution path for plotting a file (inpri data, see Chapter VIJI).

- execution path for adding, deleting, or ccpying. (Input data, see Chapter VIII).

- execution path for merging two SENPRO files. (Input data, see Chapter VIII).

- execution path for mode conversion unformatted to formatted. and reverse. (Input data, see Chapter VIII).

- execution path for listing profile titles in descending order of the absolute magnitude of $A=\sum_{i=1}^{N G} r_{i}$, where

$C_{\mathfrak{i}}$ is the sensitivity cuefficient for group $\mathfrak{i}$. (Input data, see Chapter VIII).

- execution path for normalizing the sensitivity coefficients in a file. (Input data, see Chapter VIII).

- execution path for modifying the MAT-MT control record. (Input data, see Chapter VIII).

- execution path for placing the sensitivity coefficients in a file on a user supplied group structure. (Input data, sec Chapter VIII). 
EUIT

SUM

$=$ SEITIAEL

=COIEKT

= COVEPX

LIST

ADD

DELETE

MERGE

CONYERT

FIX

EDIT

-CAVALIER

$=$ COVERS

=UNCOVER

=ADJUST

NUTCRCKR
- execution path for selectively editing or sopying a file. (Input data, see Chapter VIII).

- execution parh for suming all profiles in a file and protuci, a new file. (Input data, see Chapter VIIi):

- perturbation analysis. (Irput data, see chapter IX).

- format conversion of COVE $2 X$ files. (Input data; see Chapter $x$ ).

- Coverx Service Hodule.

- execution path for listing a fle. (Input data, see Chapter in ).

- execuicion path for addiig to a file. (Inplit data, see Cliapter XI).

- execution path for deleting from a fi?e. (Input data, see Chapter XI).

- execution path for merging two files. (Input data, see Chapter XI).

- execution path for format conversinn. (Iriput data, see Chapter XI).

- execution path for record modification. (Input datā, see Chapter XI).

- execution path for selectively editing or copying a file. (Input data, see Chapter XI).

- Nuclear Datã Uncertainty Analysis, differential data only. (Input data, see Chapter $X$ ).

- prepares input for UNCOVER. (II:ïit data, see Chapter XIII).

- uncertainty analysis and least squares adjustment taking into account results from integral experiments. (Input data, see Chapter XII).

- cross section modification (input data, see Chapter XIV).

- Nuclear data accuracy requirement determination. (Inpút data, see Chapter XV).

The modules are executed in the order the = MODNAME cards are encountered and the execution paths are executed in the order the path names are encounteres. 
1. C. R. Weisbin, J. H. Marable, J. L. Lucius, E. M. Oblow, F. R. Hynatt, R. H. Peelle, and F. 6. Peray, Application of FORSS Sensitivity and Uncertainty Methodology to Fast Reactor Benchmark Analysis," ORRL/TM5563 (1976); RSIC Code Collection CCE-334/FORSS.

2. C. R. Heisbin, P. D. Sorá, R. E. MacFarlane, D. R. Harris, R. J. LaBauve, J. S. Hendricks, and J. E. Hhite, "MIMX, A Multigroup Interpretation of Auclear Cross Sections from ENDF/B," Los Alanos Scientific Laboratory (LA-6.186-MS); see also, Trans. Am. Mucl. Soc. 16, 127 (1973).

3. H. J. Cavis, M. B. Yarbrough, and A. B. Bortz, "SPHIKX, A One-Dimensionat Diffusion and Transport Nuclear Cross Section Processing Code," UARD-XS-3045-17 (1977).

4. N. M. Greene, J. L. Lucius, L. M. Petrie, U. E. Ford, III, J. E. White, R. Q. Uright, "AwPX: A Modular Code System foe Cenerating Couplcd Multigroup Neutron-6ama Libraries from ENOF/B," OR/L/TH-3706 (Karch 1976); RSIC Code Collection PSR-063/ANPXII.

5. C. C. Haynes, -The AXMIX Program for Cross Section Mixing and Library Arrangement, ORNL-CF-74-12-2 (1974); RSIC Code Collection PSR-75/AXMIX.

6. B. M. Caimichaei, "Stanciara Interface Files arid Procedures for Reactor Physics Codes, Version III," LA-5486-MS (February 1974).

7. R. J. Barrett, R. E. Macfarlane, and R. M. Boicourt, Los Alamos Scientific Laboraotry (to be published).

8. H. H. Engle, Jr., "A Users Manual for ANISH, A One-Dimenstonal Discrete Ordinates Transport Code with Anisotropic Scattering," K-1693, Computing Technology Center, Oak Ridge Gaseous Diffusion Plant (1967); RSIC Code Collection CCC-254/ANISN-ORNL.

9. M. K. Drake, "Datd Formats and Procedures for the EMDF Neutron Cross Section Libary," Brookhaven National Laboratory report BNL-50274 April 1974 Revision).

10. C. R. Weisbin, E. M. Oblow, J. Ching, J. E. White, R. Q. Wright, and J. D. Drischler, "Cross Section and Method Uncertainties: The Application of Sensitivity Analysis to Study Their Relationship in Radiation Transport Benchmark Problems," ORNL/TM-4847 (ENDF-218) (August 1975): see also RSIC Code Collaction PSR-93/PUFF.

11. J. L. Luctus and $f$. R. Lieisbin, "Interface Specifications for Sensitivity Profiles (SEMPRO) and Covartance Fties (COVERS)," Presentation to the Comm ttee on Computer Code Coordination, Los Alamos Scientific Lasoratory (May 1976). 
1E. M. A. Rhoades and F. R. Mynatt, "The DOT III Two-Dimensional Discrete Ordinates Transport Code," ORRM/TH-4280 (September 1973); RSIC Code Collection CCC-209/00T III.

13. D. R. Vondy, T. B. Fouler, and G. H. Cunninghan, MenturE: A Code Block for Solving Mu?tisroup Neutronics Problems Applying the FiriteDifference Diffusion-Theory Approximation to Neutron Transport," ORML-5062 (Octciser 1975); RSIC Coki Collection MUC-052/VERTURE.

14. R. L. Childs, D. E. Bartine, and U. U. Engle, Jr., Trans. An. Mucl. Soc. 21, 542 (1975).

15. J. L. Lucius, "The SENPRO Service Module of the FORSS System," see Chapter VIII.

16. J. H. Marable, J. J. Drischler, and C. R. Weisbin, "SENDIN and SENTINEL: Twis Computer Codes to Assess the Effects of Nuclear Data Changes," ORML/Th-5946, ENDF-250 (July 1977), see Chapter IX.

17. J. D. Drischler, J. H. Maráble, and C. R. Meisbin, "COVERT and CAVALIER: Two Computer Codes for Reactor Performance Uncertainty Estimation lising SENPRO Sensitivity and COHERX Covariance Files," ORML/TK-6078, ENDF-256 (August 1978), see Chapter $X$.

i8. UNCOVER is a revision of the AMARA program permitting the solution of problems with a large number of energy groups. See also A. Gandini and M. Petilii, "AMARA: Code Using the Lagrange. Multiplier Method for Nuclear Data Adjustment, "private commiration, M. Salvatores (1975), see Chapter XII.

19. See reference 1, p. 102 . 
CHAPTER II. THE IMPROVED FIDO INPUT SYSTEM

J. H. Marble

$c$ 


\section{THE IHPROVED FIDO IMPUT SYSTEM}

The FIOD input method was cevised for entering data into large arrays, using patterns of repetition and symetry wherever possible. This method was designed by Ward Engle and Mayne Phoades using the input wethod of the Los Alanos F.OCO coding system as a me?. It was first applied to the DTF-II code, and since that time FIOO has been applied to other codes. For these a free-field option and other features were incorporated.

Recently extensive improvenents were made by James Marable. Miese improvements include the reading of for tted or unformatted pieces of arrays from various $1 / 0$ devices, reading hollerith characters, reading numbers to an arbitrary base (e.g., octal, binary, and hexidecimal), modifying (by mitiplication, etc.) numbers already in storage, entering double precision arrays, and other changes. It is important to note that these improvements have been incorporated without changing the previous definitions. Old FID input decks will still be read correctly!

Each FIDO cail for input allows data to be read into a "block" until a delimiter ' $T$ ' instruction operator appears in the input. Such a "block" is partitioned into consecutive arrays, each of which is numbered according to its order in the block. Input data is read into an array by first designating the array number and the data type - integer, floating point, or double precision. For each FIDO input call the order in which arrays are designated is of no importance, and an array can be designated an arbitrary number of times in a single FIDO input call.

For example, an array can be filled with zeroes using a special option, al,t chen a few scattered locations can be changed by designating the array again and reading in a partial set of new data for that array. If no entries to the arrays in a block are needed, the ' $T$ ' delimiter alone satisfies the FIDO input call.

Three major types of FIDO input are available: fixed-field input, 
free-field input, and user-format input. Only the free-field option is discussed hero. Free-field FIO0 reads data into an array by units called fields. To read data into an array, an array designator field must first appear. Data defined by successive fields are then entered until the required nuber of entries has been accounted for.

In entering daca, it is convenient to think of an "index" or "pointer" wich specifies the array location into which the next data entry is to go. The pointer is alwajs positioned it array location 1 by entering the array designator field. The pointer subsequently moves through the array according to the data operators chosen.

Each field is broken up into three subfields. However, it is not always necessary that all three subfields appear explicitly in the input. Frequently only one or two subfielós are given, and any wissing subfield is ioplied or is simply not required.

The three subfields wich make a field are $M, 0 p$, and $M 3$. The first subfield $M I$ is an integer and the second subfield $O p$ is a character specifying a typo of input operation. The third subfield $N 3$ is an integer, a floating point number, a double precision number, or a string of hollerith characters according to the array type and the input oneration. FIDO input fields must not extend beyond the first 72 characters of a card image, and all subfields of a field must be on the same card.

The first subfield is an integer $M 1$ wich has a preset default value of zero. (Operations denoted by $Z$ and $R$ are exceptions in that the default value of $M I$ is unity.) If the first subfield appears explicitly in an input field it must be followed immediately by the second subfield operation character without an intervening space. Negative integers are al lowed fr $N$ for some of the operations defined by the second subfield.

The third subfield N3 may have one of the following five forms:

(1) An integer (positive, negative, or zero) of any magnitude allowed by the computer.

(2) A decimal nuniter with an arbitrary number of digits. If no decimal is given explicitly it is assumed to follow the last digit.

(3) A floating Doint number wich consists of a decimal number (as defined above) followed by an exponent indicator E, D, 
t, or -. Any of these four exponent indicators may be followed by a single space before the exponent itself is given.

(4) A floating point nuber (with or without an exponent) io an arbitrary base $b(2$ through 36$)$. For this purpose digits are chosen from $0,1,2, \ldots . . .9, A, B, C, \ldots$ - $Z$ as required. If the base $b$ is greater than 12 , the character $D$ is no longer valid as an exponent indicator, and if the hase $b$ is greater than $13, E$ is no longer valid as an exponent indicator. Of course, + and - are sti?l available as exponent indicators. The subfield entry consists of the base b (using the base 10) followed by the single character " $X$ " and then the floating point numer itself. Ho blank may appear, between the base $b$ and the ' $X$ ', but any number of blanks may occur between the ' $X$ ' and the floating point entry. If an exponent appears, it is also to the base $b$ and gives powers of the base $b$. Each subfield is assumed to be the base 10 unless the base is given explicitly in that subfield.

(5) A string of alphanumeric or hollerith characters. This is used only with the operation sharacter ' $H$ ', and the first subfield $N 1$ is the number of such characters.

The second subfield consists of a single character, except for the three array-designator characters \$\$, **, and \$, which when entered in pairs indicate that data is to be entered using the free-field input option.

Tre characters and the corresponding operation instructions are listed in Table 1. Operator characters with superscript 'a' dcnote operations which ignore the first, subfield value N1. Operator characters with a superscript ' $b$ ' denote operations for which it is not possible to enter a third subfield (using free-ffeld input). These characteirs terminate the field, and a new field starts immediately reagardless of whether there is a space or not. Except for these operations a field is terminated by a space following the third subfield.

In general, no space is allowed between the first and second subfields, 
and spaces are allowed but are not required between the second ard third subfields. Setween fields spaces are allowed and one is required (except for above exceptions associated with superscript ' $b$ ').

Typical FIDO input is show in Figure 1. The resulting contents of the arrays is given by the output shown in Figure 2. 
Table 1. Cilaracters and Their Corresponding Operation Ins tructions

\section{Character Operation Instruction}

\begin{tabular}{|c|c|}
\hline & $\begin{array}{l}\text { Designate array } N 1 \text { to be an integer array and set the } \\
\text { pointer to the first location in array } M 1 \text {. When inter- } \\
\text { polated, etc., numbers are always rounded off to the } \\
\text { nearest integer value. }\end{array}$ \\
\hline & $\begin{array}{l}\text { Designate array } M I \text { to be a floating point array and set } \\
\text { the pointer to the first location in array } N \text {. }\end{array}$ \\
\hline & $\begin{array}{l}\text { Designa ie array } M \text { to be a double precision array and } \\
\text { set the pointer to the first location in i.ray } \mathrm{NI} \text {. }\end{array}$ \\
\hline & $\begin{array}{l}\text { Skip to the next card ignoring all comments foliowing } \\
\text { the slash. }\end{array}$ \\
\hline$(\text { blank })^{a}$ & $\begin{array}{l}\text { Enter the third and only nonblank subfield into the } \\
\text { location indicated by the pointer and then advance the } \\
\text { pointer by } 1 \text {. }\end{array}$ \\
\hline & $\begin{array}{l}\text { Enter } \mathrm{O}|\mathrm{N} 2| \text { times and advance the pointer by }|\mathrm{N}| \text {. If } \\
\text { zero or blank is entered for } \mathrm{Nl} \text { it is replaced by } 1 \text {. }\end{array}$ \\
\hline & $\begin{array}{l}\text { Enter the third subfield | } \mathrm{NL} \mid \text { times, and with al ternat- } \\
\text { ing sign if } \mathrm{NI} \text { is negative. Increase the pointer by } \\
\text { IMI. If a zero or blank is entered from } N \text {, it is } \\
\text { replaced by } 1 \text {. }\end{array}$ \\
\hline & $\begin{array}{l}\text { Determine } \mathrm{Nl}+2 \text { numbers by linear interpolation starting } \\
\text { with the third subfield of this field and ending with } \\
\text { the third surfield of the next field. Enter the first } \\
\mathrm{Nl}+\mathrm{l} \text { numbers. }\end{array}$ \\
\hline & $\begin{array}{l}\text { Terminate this call for FIDO input and roturn to the } \\
\text { calling program. }\end{array}$ \\
\hline
\end{tabular}


Table 1. (continued)

\section{Character : Operation Instruction}

$L \quad$ Determine $M+2$ numbers determined by logarithmic interpolation starting with the third subfield of this field and ending with the third subfield of the next field. Enter the first $N 1+1$ numbers. (The logarithms of the numbers entered are uniformly s,aced.)

Repeat the sequence of |N3| numbers immediately preced-
ing the puinter $|N|$ more times, multiplying on each
repetition each number of the sequence by 10 or 0.1
according as $N 3$ is positive or negative. If $N 1$ is nega-
tive the sign of the sequence changes on each repetition.
Repeat the sequence of |N3| numbers immediately preced-
ing the pointer $|N 1|$ more times. If $N 1$ is negative
change the sign of each number of the sequence on each
repeat. If $N 3$ is negative reverse the order of the
sequence for each repetition. The pointer is fir.ally
advanced by $|N 1| *|N 3|$.

$N$ This is equivalent to the operation $Q$ b! th a first subfield $\| 1$ and a third subfield - $|N 3|$.
$M$ This is equivalent to the operation $Q$ with a first sub- field - $|N 1|$ and a third subfield N3.

$c^{a, b} \quad$ Print the pointer value of the last array item entered.

This is one less than the pointer position.

If $N ?>0$ the print trigger is turned on.
If $N 1=0$ the crint trigger if ilipped.
If $N 1<0$ the print trigger if turned off. When the print
trigger is on, each card image is printed as it is read.


Table 1. (continued)

Character Operation Instruction

$\mathrm{s}^{b} \quad$ Add $\boldsymbol{N}$ to the pointer value. $M$ may be negative thereby
desc easing the pointer value.

\begin{tabular}{|c|c|}
\hline$A^{a}$ & Place the pointer at $\mathbf{N 3}$. \\
\hline $\mathbf{F}$ & $\begin{array}{l}\text { Fill the remining lo:ations of the array with the third } \\
\text { subfield entry. If } \mathrm{Nl} \text { is negative the entries alternate } \\
\text { in sign. Set the pointer after the last location. }\end{array}$ \\
\hline$E^{a, b}$ & $\begin{array}{l}\text { Skip over the remainder of the array by placing the } \\
\text { pointer after the last location. }\end{array}$ \\
\hline H & $\begin{array}{l}\text { Enter the N1 hollerith characters which are in the third } \\
\text { subfield. Advance the pointer by the number of words } \\
\text { requireo to store tilese } N 1 \text { characters. } \\
\text { (number of words = (N1+NCPW-1)/NCPW where NCPW is the } \\
\text { number of characters per word). }\end{array}$ \\
\hline $\mathbf{G}$ & $\begin{array}{l}\text { Read } N 3 \text { words from } 1 / O \text { device with data set reference } \\
\text { number } N 1 \text { according to the format to be specified in the } \\
\text { next field which is hollerith. If the next field is } D \\
\text { or holleriti blank the field is unformatted. Advance } \\
\text { the pointer by } N 3 \text {. }\end{array}$ \\
\hline
\end{tabular}

If $N I$ is positive, change the input unit so as to read the succeeding card images from unit $N I$ until a delimiting $T$ operation appears (or until a similar $Y$ instruction appears). If $\mathrm{N} 3>0$ change all FIDO edit to unit $\mathrm{N} 3$. If $N 3<0$ change card image listing (see ' $O$ ' operation) only to unit $|\mathrm{N}| \mid$. After a delimiter $T$ appears the next call to FIDO resets the input and output unit numbers to the original vaiue.

Ordinarily FIDO enteis data - interpolated, sequence
repeist, etc. - by entering each "raw entry" into the
profer location obliterating the previous "old number"


Table 1. (continued)

Character Operation Instruction:

in that location. By means of the operation denoted by charicter ' 6 ' F. DO changes its mode of entering data according tc the value of the first subfield NI preceding the operation character ' $\rho$ '. The various manipulations are performed on the "old number" in storage and the "raw entry" in order to obtain the final number which is stored. Let $A$ be the "old number" previously stored, let B be the "raw value" determined by the usual FIO0 entry. We have the following possibilities fer the number finally stored according to the value of $\boldsymbol{M}$.

$\begin{array}{cc}\frac{N}{0} & \frac{\text { Number Entered }}{B} \text { (the default mode) } \\ 1 & A+B \\ 2 & A-B \\ 3 & A \star B \\ 4 & A / B \\ 5 & B / A \\ 6 & B^{\star E X X(A)} \\ 7 & B^{\star} \operatorname{LN}(A) \\ 8 & \operatorname{EXP(B)} \\ 9 & \operatorname{LN}(B)\end{array}$

Each time an array is designated by an array designator field the default mode $(N T=0)$ is l'eactivated causing raw data to be.entered directly into array storage. 
Figure 1. Data for Three Calls for FID0 Input on Unit 5

C1GO,FTISFOOL Do

coso69718329999999999999

18

PTHIS IS THE FIRST CARD READ IN THE FiHST CALL FUH FIOU JPOUT

o THIS Flips THE DAINT TRIGGER.

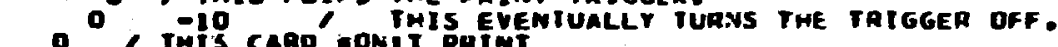

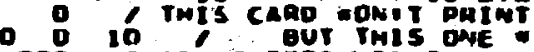

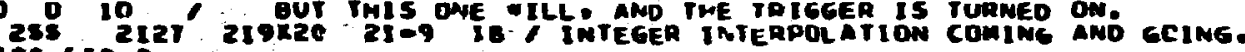

10.10108 201000-300.0001.0011111. 701.0-15501.0 602.0 00i. 902. E

360 IH THIS IS IT

SE Togio

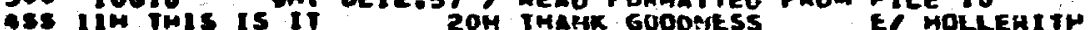

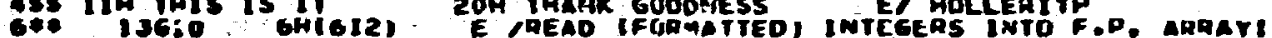

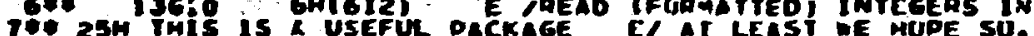

I2e日

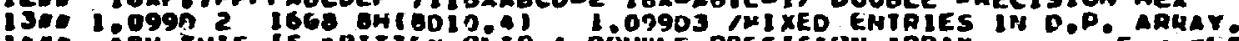

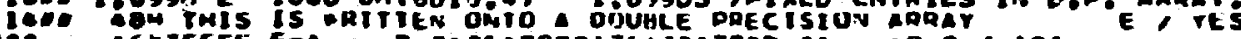

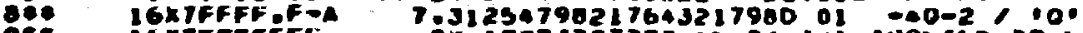

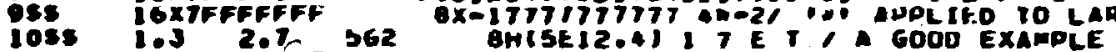

16.8 0.300 .

fOATA ON INE DAEYIOUS CAPD WEAE REAO INTO DOSITIONS SE OF THE IOSB ARAAY

fTHIS IS THE SECOND CAPO READ IN THE SECONC CALL FOR Fido INPUT

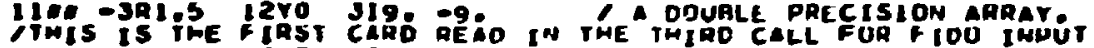

1. 999.9 9123.9 12349. A FE* SIMPLE F.D. MUVIAERS.

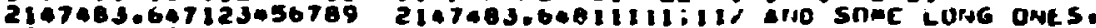

- $9990 \mathrm{C}^{2} \mathrm{~T}$ AND UNE MITH A SWACED EMPONENT

I6XF.TFFFFFF IGXABCDEFO-AF THESE AHE SOWE MEX NUMEERS

2A1011.0110011 F2X1001.10E-100I SONE GINARY NUWEES.

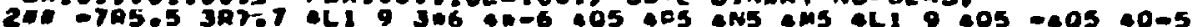

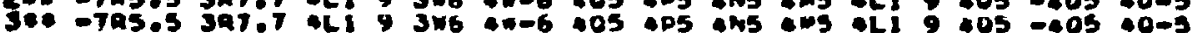

$4552127219 \times 2021-9$ 20

21474836472147403648

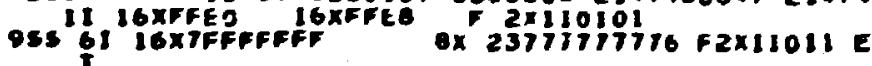

$1 \%$

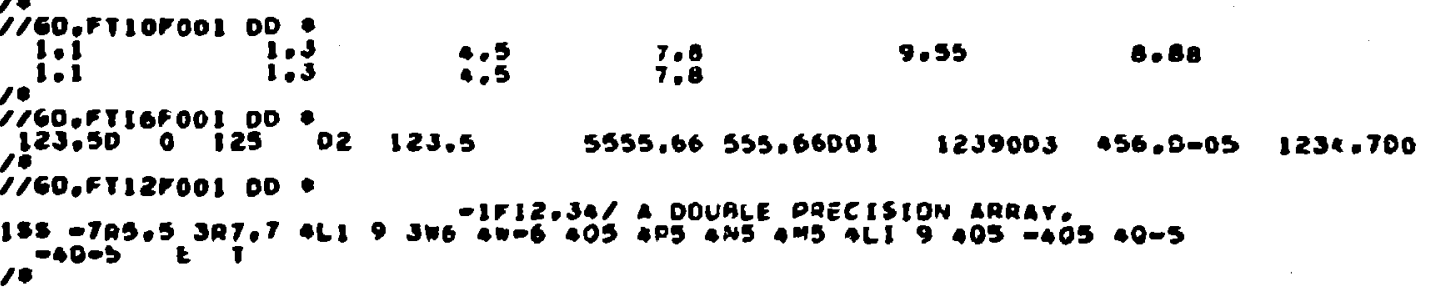


II -12
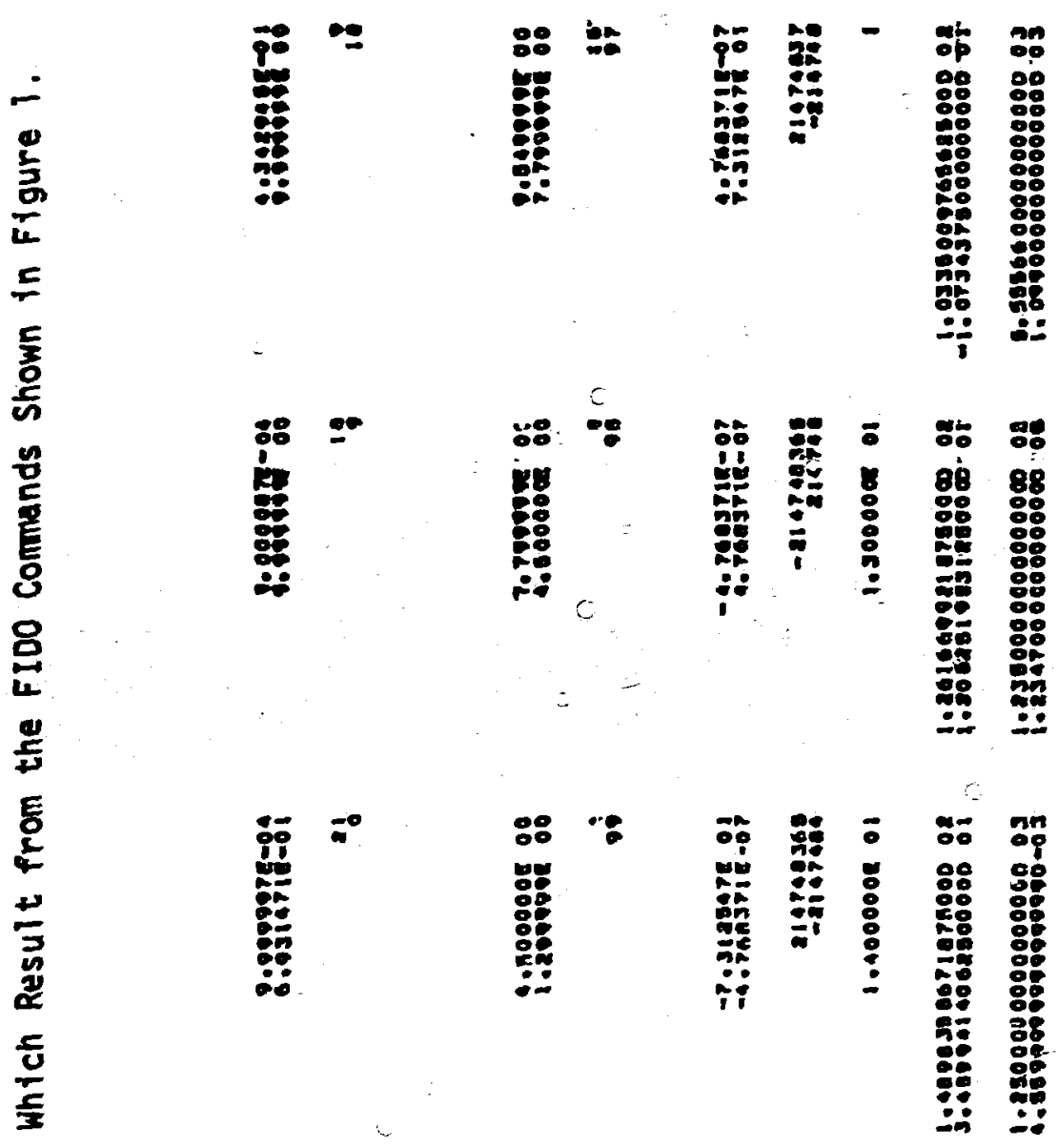

옹

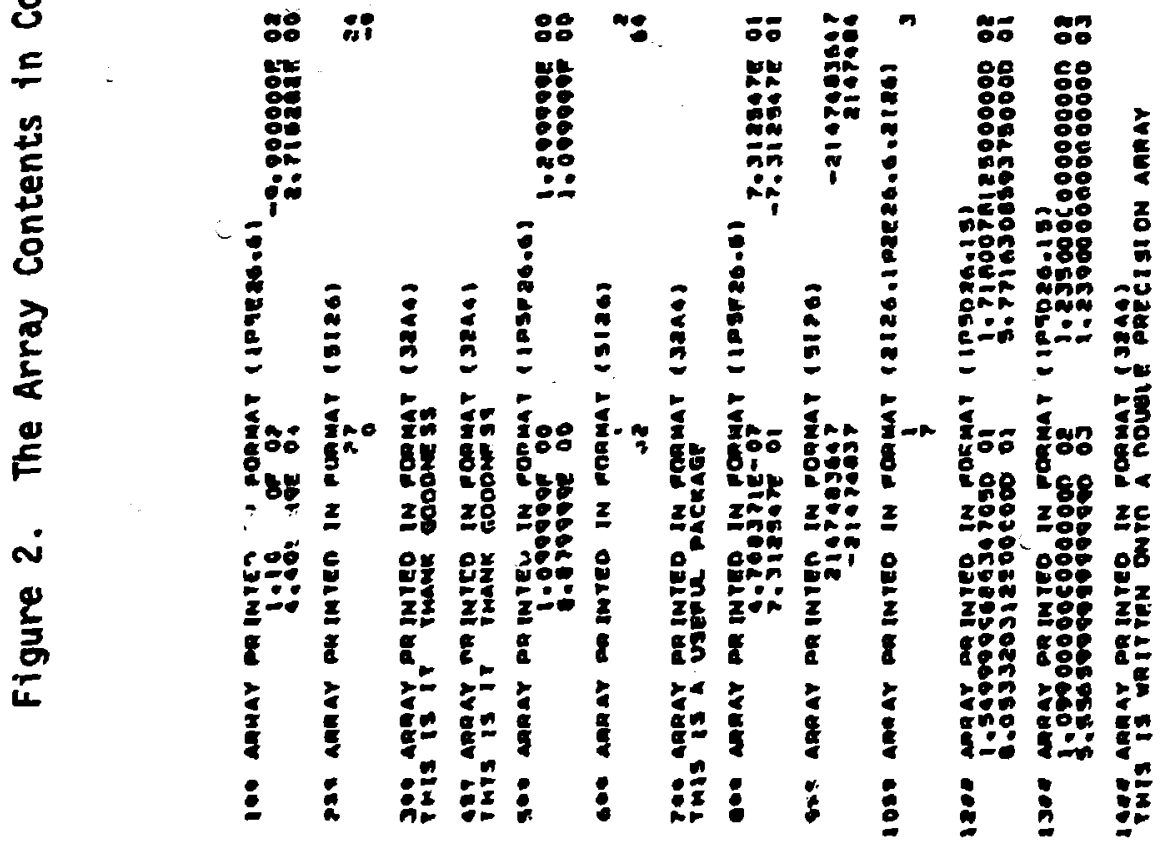




\section{II-13}
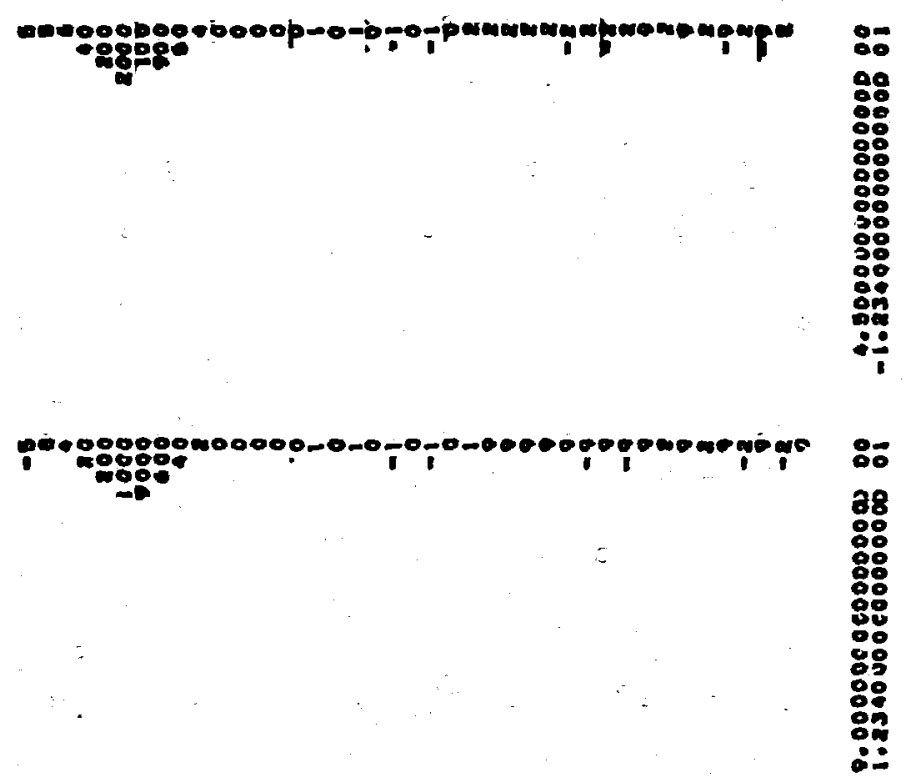

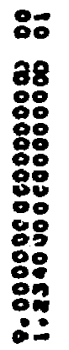

얼

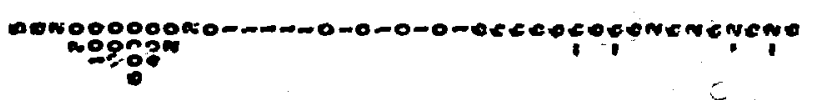

웅

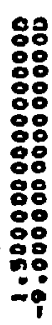

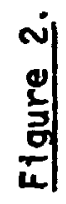

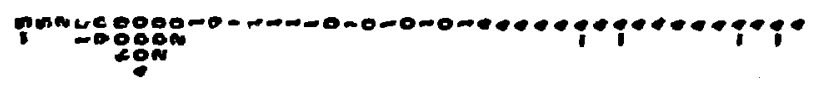

:ะ $c$

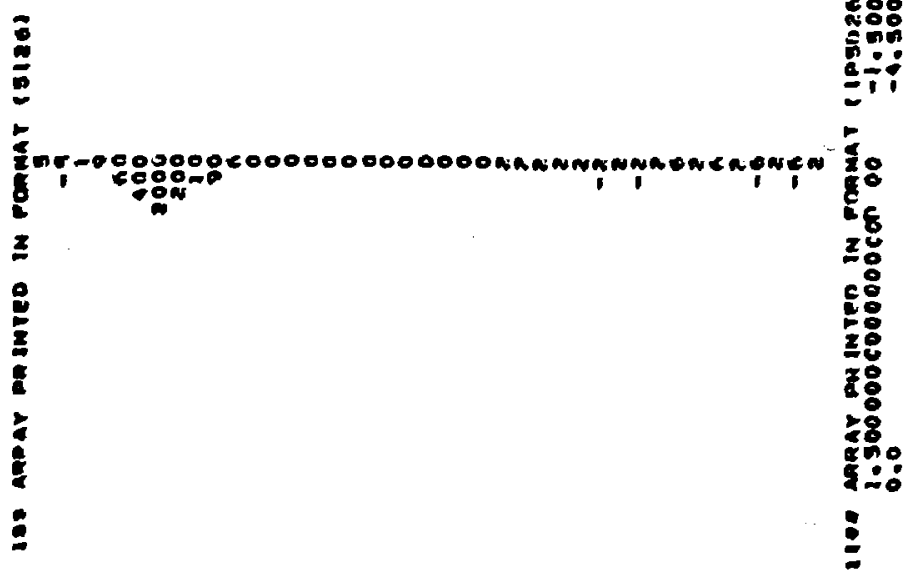




\section{II-14}

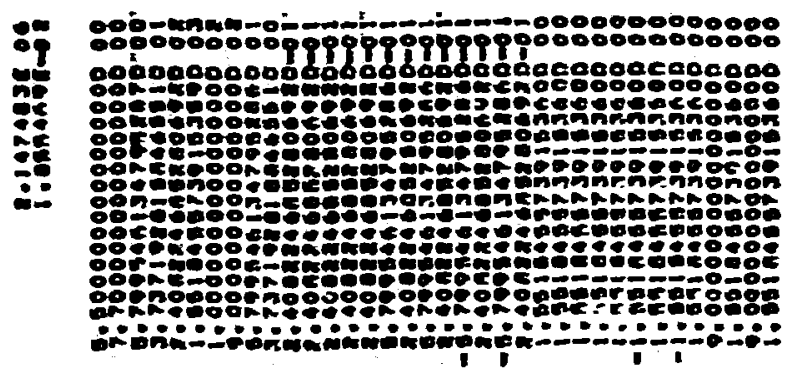

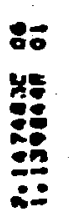

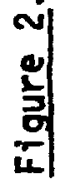

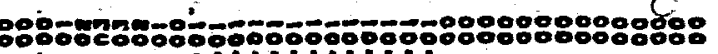

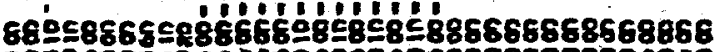
\%1 :80:0 :27n:- L \&.5.

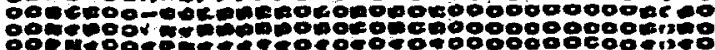
:07

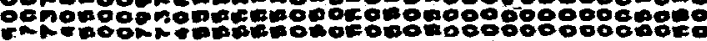

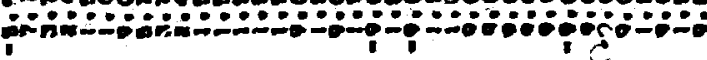
:08-xcun

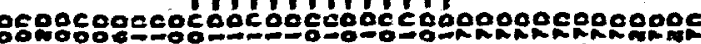
○ำำ

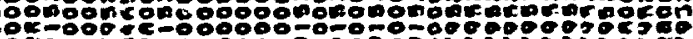

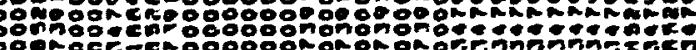
:ơำ

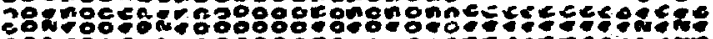

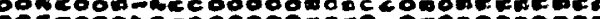

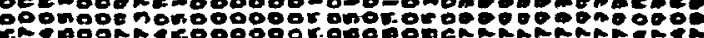

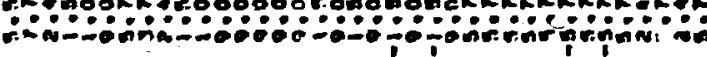

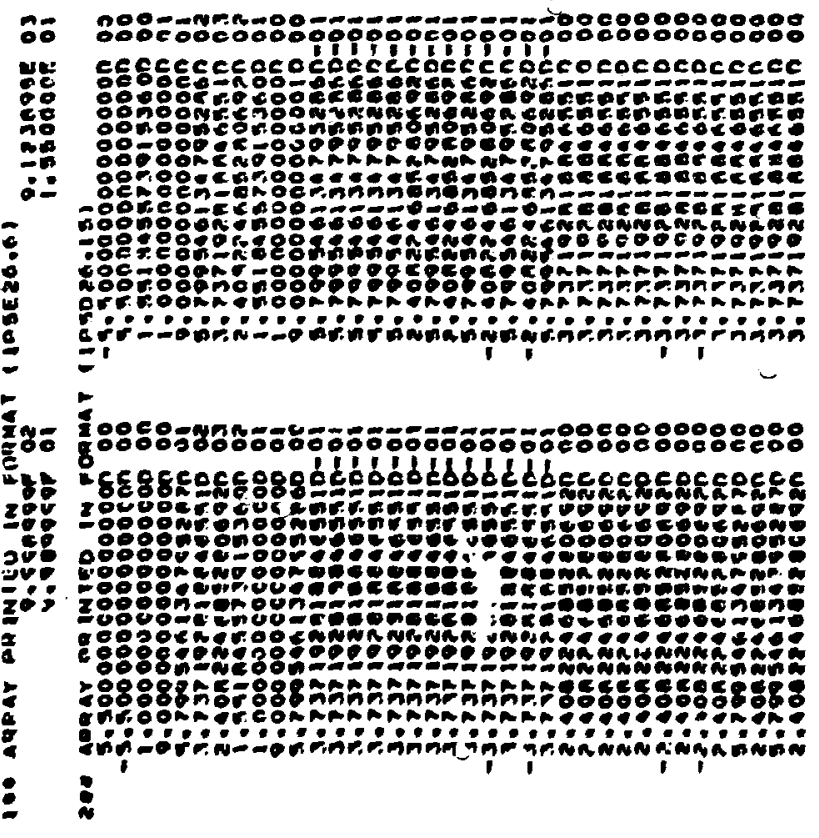




\section{II-15}

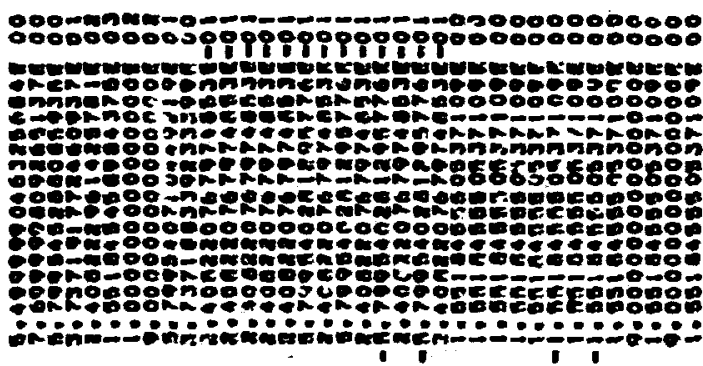

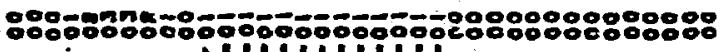
qEy

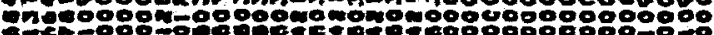

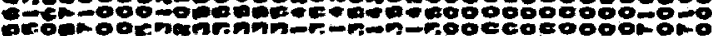

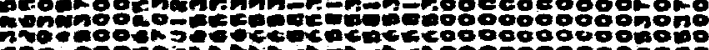

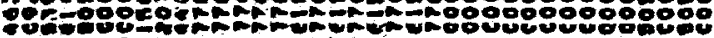

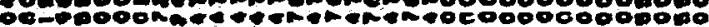

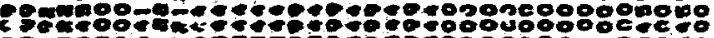
CE-FE8: 7 -

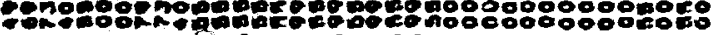

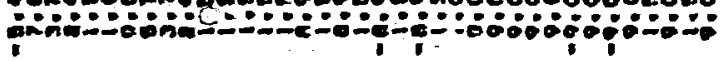

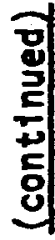

인

800-ตทวผ

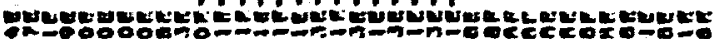
Eทㅁo8:

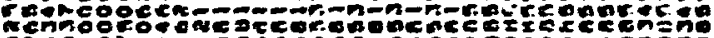
jecoo.

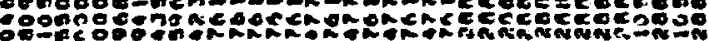

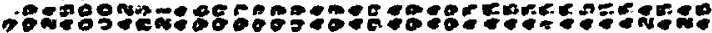

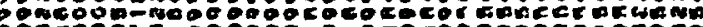
ใcyno:

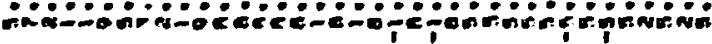

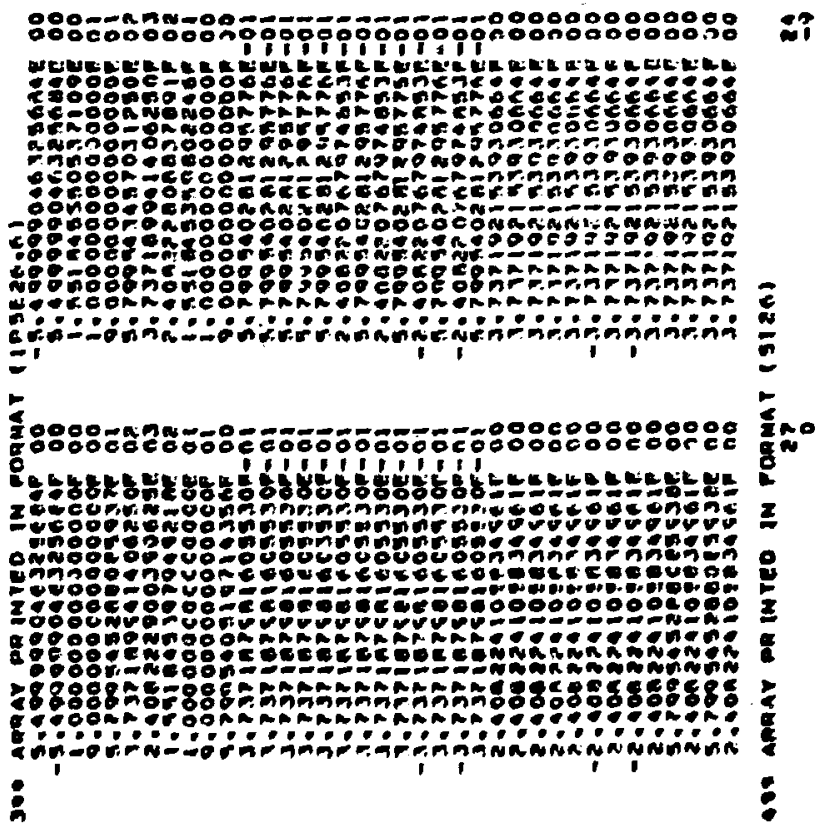

e.

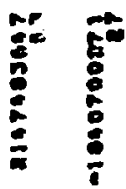

an

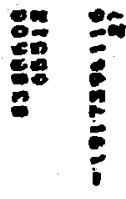

ai

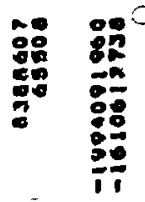

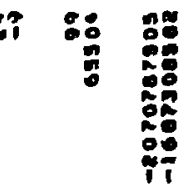

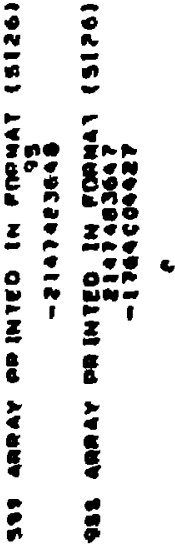


C.

CHAPTER III. THE ANISH, AMPX, ISOTXS TO MATXS TRANSLATORS

C J. L. Lucius

( 


\section{CHAPTER III}

Table of Contents

Page No.

A. Introducticn ................... III-3

B. ANISN to MATXS Cross Section Translator ........ III-3

1) Input [escription. .............. III-4

2) Sample Inpit for ANISN to MATXS Translator . . . . III-6

C. AMPX to MATXS Translator ............... III-6

1) Input Description ............... III-8

2) Sample Input for AMPX to MATXS Translator ..... III-8

D. ITOM and PHATXS ................. III-8 


\section{A. Intioduction}

To calculate sensitivity coefficients the JULIETi module requires access to partial cross section sets. This is achieved with the profosed CCCC $^{2}$ file HATXS? which is specified in Section ic of Chapter VII. However, many cross section sets of interest are available in other formats Surh as ANISN ${ }^{4}$, AMPX $^{5}$, and ISOTXS ${ }^{2}$. The translation capabilities described in this chapter make these cross section sets available in the MATXS format.

\section{B. ANISN to MATXS Cross Section Translator}

Many problems do not require the translation of every material on the ANISN file to MATXS format. The trinslator includes a selective copy capability which prodices a subset of the original ANISN file containing only the materials to be transiated.

The ANISN cross section format uses numerical material identifiers and the reaction iype is defined as a function of table position. The user must provide the translator enough information for materials and reaction types on the MATXS file to be identified by hollerith character strings (words). An input card is prepared for each material on the ANISN file containing the name to be assigned to the material on the MATXS file. The order in which these cards are input is crucial. Material $I$ on the ANISN file goes on the MATXS file with the name found on the first material name card input to the translatur, etc. This oneto-one correspondence must be correctly established or disaster is assured. Reaction type identification is built into the translator as a function of the ANISN table position (IHT) of the total cross section. 
When IHT $=3$, the translator assumes the following correspondence between table position and reaction tyce:

\begin{tabular}{|c|c|c|}
\hline $\begin{array}{c}\text { ANISN } \\
\text { TABLE POS:TION }\end{array}$ & REACTION & $\begin{array}{l}\text { PEACTION NAMAE } \\
\text { CII UATYS }\end{array}$ \\
\hline 1 & $z_{a}$ & ABSORP \\
\hline 2 & $v_{f}$ & NLEIS \\
\hline 7 & $s_{t}$ & TOTAL \\
\hline
\end{tabular}

When $I H T=5$, the following correspondence is assumed:

$\begin{array}{lll}1 & x & \text { CHI } \\ 2 & \sigma_{f} & \text { FIS } \\ 3 & \sigma_{a} & \text { ABSORP } \\ 4 & v \sigma_{f} & \text { NUFIS } \\ 5 & \sigma_{t} & \text { TOTAL }\end{array}$

The translator calculates $\sigma_{c}=\sigma_{a}-\sigma_{f}$ only when IHT $=5$ and identifies the reaction as MT101 on MATXS.

For those specialized cruss section files for sensitivity analysis where a single reaction type has been placed in the ANISN format, the identification of the reaction type as a function of table position cannot be accomplished and the user must expand the matirial identification card to also identify the reaction type.

1) Input Description

iss Integer Parameters [11]

i. NNG - Number of groups

2. IHT - Position of $\sigma_{t}$ in cross section table

3. ITL - Length of cross section table

4. IGG - Position of ${ }^{\mathrm{g} g g}$ (self scatter)

5. NL - Order of scatter, every material or the ANISN file must have the same order of scatter, enter 1 for PO, 4 for $P_{3}$, :LC.. 
6. NMAT - Number of materials an AISN file. Pn>0 are not counted ds miterials for this inpui. Set MMAT = -NMAT if ANISN cross sictions are on cards. See description of $2 £ 5$ card.

7. IVERS - MATXS Fiie version number. IUERS can be any number the user selects.

8. ISIG - Unit number of ANISN file (default = 4)

9. NN - Unit number of MATXS file (default = 8)

10. IOLT - Unit number of copy of ANISN fi?e (default = 35)

11. LECT - Set to 0 , reserved for future option

$25 \$$ Integer Parameters [100]

NL
NL

- ANISN material identifiers, an; material identitier (ID) on the ANISN file satisfying the test $N L \geq I D<N U$ will be copied from Unit ISIG to Unit IOUT. A maximum of 49 pairs of $N L$, NU may be entered. The 2SS card may be omitted if no copy is desired. If a copy is made, N,AT, see 155 vard item 6 : mus: be equal to the number of materials copied to Unit IOUT.

FO

$T$

4** Group Boundaries [NNG +1 ] . Enter the energy group boundaries, high energy to low exergy, in units of $\mathrm{eV}$.

T

NMAT

- Fixed field cards follcw, one for each of the NMAT materials.

Columns 1-6, Hollerith identification to be assigned to each material on the ANISN file. NOTE: $P_{n}>0$ not counted as a material for this input.

Columns 7-10, blank

Columns 11-i6, if a specialized file is being processed, enter a word that identifies the reaction type, otherwise leave blank

If $($ MMAT $<0)$, i.e., cross sections on cards, prepare $5^{\star *}$ arrays. 


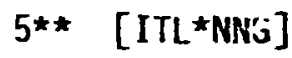

ANISN cross section cards

Repeat $5^{\star \star}$ data for each scattering order

Repeat $5^{\star \star}$ sequence for each material to te translated

End each $5^{\star \star}$ array with a $T$.

2) Sample Input for ANISN to MATXS Translito:

$=$ ANTMX

$\begin{array}{rrrrrrrr}1 \$ \$ & 100 & 5 & 105 & 6 & 4 & 7 & \text { F0 } \\ 2 \$ \$ & 9 & 36 & F 0 & T & & & \\ 4^{\star \star} & & & & & & & \end{array}$

(Group boundaries, 101 entries)

T

PU240

C PU241

U235C

U235B

$\mathrm{U} 238 \mathrm{C}$

U238B

PU239

The sample input takes seven, $P_{3}$ materials with material identifiers 9-36 from an ANISN file on Unit 4 and writes a new file on Unit 35 containing only the seven materials of interest. The seven materials are then translated from the ANISN format (Unit 35) to the MATXS format (Urit 8). Resource requirements are $270 \mathrm{~K}$ bytes of core storage, 1900 I/O operations, anci eight seconds of execution time on an IBM 360/91.

\section{AMPX to MATXS Translator}

This module will transiate AMPXS master files containing neutron data. Other arrangements must be mane for AMPX master files containing both neutron and gamma cross sections. An established option is NITAWL to ANISN then to MATXS. AMPX uses the ENDF/B numerical material (MAT) reaction type (MT) identification scheme. MATXS uses Hollerith character 
(word) identification schemes. The translator requires user input to establish a correspondence. Every material on the AMPX master is translated to MATXS. An input card is prepared for each material on the AMPX master containing the name to be assigned to the material or the MATXS file. The input order of these cards is crucial. A one-to-one correspondence between the name cards and the materials on the AMPX master must be correctly establisned. The translator establishes the following reaction type correspondence:

\begin{tabular}{cl} 
ENDF/B (MT) & MATXS \\
\hline 1 & TOTAL \\
2 & ELSCAT \\
3 & NONELS \\
4 & INSTOT \\
16 & N2N \\
17 & N3N \\
18 & FIS \\
19 & FCFIS \\
20 & SCFIS \\
21 & TCFIS \\
51 & INELOI \\
$\vdots$ & $\vdots$ \\
90 & INEL40 \\
91 & CTNUUM \\
102 & CAPT \\
452 & NUBAR \\
1452 & NUFIS
\end{tabular}

For other ENDF/B MT numbers the translator forms a MATXS six character string as follows. If the MT $=1234$ the MATXS identifier wiil be MT1234. 
1) Input Description for the AMPX to MATXS Translator. Formatted input is required rather than FIDO input.

Card 1: FORMAT (2I5)

NuW - Number of materials on AMPX file to be translated

MLD - Maximum order of scatter, enter 0 for Po, etc.

Cards 2 through NMA+1 FORMAT $(A 6,4 X, 16)$

HMATI - MATXS material description, $\$ 6$ Hollerith characters (left adjusted)

MATW - AMPX material identification number (right adjusted)

2) Sample Input for AMPX to MATXS Translator.

\begin{tabular}{lllll} 
Coluind & 1 & 5 & 10 & 12 \\
\hline $016-\mathrm{C}$ & & 3 & \\
$016-\mathrm{B}$ & & & 12761 \\
& & & 12762
\end{tabular}

The sample input translates two, $P_{3}$, AMPX materials from an AMPX master on Unit 3 to a MATXS master on Unit 8. Resource requirements are $270 \mathrm{~K}$ bytes of core storage, 6300 I 00 operations, and 40 seconds of execution time on an IBM $360 / 91$.

Unit numbers 3 and 8 are defined in the code. A more efficient and flexible AMPX to MATXS translator is under development.

D. ITOM ${ }^{3}$ and PMATXS ${ }^{3}$

ITOM translates an ISOTXS file to MATXS. PMATXS lists a MATYS file. No input data are required for either modu?e. 


\section{REFERENCES}

1. J. L. Lucius, E. M. Oblow and G. H. Cunningham, III, "A User's Guide for the JULIET Module of the FORSS Sensitivity and Uncertainty Analys is Code System," ORRL/TM-6594 (ENDF-275) (February 1979).

2. B. M. Carmichael, "Standard Interface Files and Procedures for Reactor Physics Codes, Version III," LA-5486-MS (February 1974).

3. R. J. Barrett, R. E. MacFarlane and R. M. Boicourt, Los Alamos Scientific Laboratory (to be published).

4. H. H. Engle, Jr., "A Users Manual for ANISN, A One-Dimensional Discrete Ordinates Transport Code with Anisotropic Scattering," K-169?, Computing Technology Center, Oak Ridge Gaseous Diffusion Plant (1967).

5. M. M. Greene, J. L. Lucius, L. M. Petrie, H. E. Ford, III, J. E. White, R. O. Wright, "AMPX: A Modular Code System for Generating Coupled Multigroup Neutrori-Gamma Libraries from ENDF/B," ORNL/TM-3706 (March 1976). 
CHAPTER IV. THE FORSS DATA REPOSITORY

J. L. Lucius 
Page Ho.

A. Data Interchange Between FANISK and JULIET . . . . . IV-3

1) Initial FANISH Runs . . . . . . . . . . . . IV-6

2) JULIET Generalized Source Runs ............ IV-6

3) Fl'sx Preparation for Sensitivity Calculations. : - - IV-7

4) Normalization and Response Parameters for JULIET Sensitivity Calculations ............ IV-7

B. FORSS Manager (FMANG) . . . . . . . . . . . IV-8

1) FANISN-JULIET Data Transfer ............ IV-8

2) Error Recovery ................. IV-9

3) Repository Copy ................ IV-10

4) Repository List ............... IV-10 


\section{IV-3}

A. Data Interchange Between FANISN and JULIET

The solution of a FORSS problem requires a significant interchange of information between FANISN and JULIET. Under DRIVER control this process has been somewhat automated. The following types of data have been assigned a numerical identification and placed on a sequential I/0 device which is referred to as a repository:

ID

1 FANISN forward angular ilux

3 FANISN calculated multiplication factor

4 Fission term in FANISN forward equation. Results labeled as FISSION DENSITY on FANISN output

5 FANISN adjoint angular flux

6 Fission term in FANISN atjoint equation. Results labeled as FISSION DENSITY on FANISN output

7 An eight word record containing responses and nomalization parameters

IS, W1, W2, W3, W4, W5, W6, 7

IS is a signal. If IS $=1$, the data on the record are for a bilinear response (see Chapter VII, Equations 19-23).

If IS $=0$, the data on the record are for a linear response (see Chapter VII, Equations 28-30).

When IS $=1$

$W 1=\frac{\left\langle q * \mathrm{H}_{1} \emptyset\right\rangle}{\lambda\left\langle\nabla * \mathrm{H}_{2} \emptyset\right\rangle}$ (see Chapter VII, Equation 19). WI is used as a normalization parameter in the calculation of a worth sensitivity profi?e 
$H 2=\frac{\left\langle\beta \star H_{1} \emptyset\right\rangle}{\left\langle\theta+H_{2} \emptyset\right\rangle}$ (see Chapter $\forall I I$, Equation 19). H2 is the bilinear response.

$H 3=\left\langle\|^{\prime} H_{1}\right\rangle$ numerator of bilinear response, used as a nomalization parameter in worth sensitivity (see Chapter VII, Equation 39).

W4 $=\left\langle\theta^{\star} \mathrm{H}_{2}\right\rangle$ denominator of bilinear response, used as a nomalization parameter in worth sensitivity (see Chapter VII, Equation 40).

$W E=\frac{\left.\left\langle\theta^{*} H_{1}\right\rangle\right\rangle}{\left\langle\theta^{*} H_{2} \phi\right\rangle}\left[\frac{.6023}{N}\right]$. differential worth

where $N$ is the number density (atoras/bn-cm) of the material of interest.

H6 $=\lambda\left\langle\theta^{\star} \mathrm{H}_{2} \phi\right\rangle$, the normalization parameter for $k$ sensitivity. $1 ! 7$ = IANISN calculated multiplication factor.

When IS $=0$

$W 1=\frac{\left\langle\mathrm{H}_{1}\right\rangle}{\left\langle\mathrm{H}_{2} \emptyset\right\rangle}$ the linear response (see Chapter VII, Equation 23). $\left.W_{2}=\left\langle H_{1}\right\rangle\right\rangle$ numerator of linear response, used as a normalization parameter in the calculation of a reaction rate ratio sensitivity (see Chapter VII, Equation 35).

$W 3=\left\langle\mathrm{H}_{2} \emptyset\right\rangle$ denominator of linear response, used as a normalization parameter in the calculation of a reaction ratio sensitivity (see Chapter VII, Equation 36).
W4
W5
W6
W7 
9 Generalized adjoint source calculated by JULIET. For bilinear source see Equation 25, for linear source see Equation 31 JULIET Users Guide Chapter VII.

11 Generalized source normalization calculated by FANISH = $\langle v(j) s(j, g)\rangle$ (brackets imply integration over space and energy of 0 th moment source).

12 Generalized forward source calculated by JULIET. See Equation 24 JULIET Users Guide Chapter VII.

The repository has been designated by default as unit 2 . This designation can be changed by user input to the FMANG module (see p: 6 ).

FMANG is a modu'e that manages the data interchange between ANISN and JULIET. The execution of FMANG is a prerequisite to the execution of either FANISN or JULIET. FMA.IG scans the repository which contairs the numerically identified data described above, and sets up a directory of the data on the repository. The directory is updated in FANISN and JULIET when any action involving the data is taken. Both modules know the position of the repository at all times to permit access to a particular data type with reasonable efficiency. Since the refository is a sequential device, it is structured to accommodate the typical proolem with a minimum of device scanning.

A labeled common /D3MGT/ and unit 2 are used to make the directory available to points of interest throughout the system.

The direztory includes a 10-word record for each ty'ne of data that has been written on unit 2. Currently six of these words nive an assigned function and four left open for expansion.

\begin{tabular}{cl} 
Word & \multicolumn{1}{c}{ Function } \\
1 & Assembly identification number \\
2 & Response related identification number \\
3 & Data type identification number \\
4 & Block number \\
5 & Number of records \\
6 & Record length
\end{tabular}




$\begin{array}{rr}7 & 0 \\ 8 & 0 \\ 9 & 0 \\ 10 & 0\end{array}$

Each data block on the repository is immediately preceded by its descriptive 10-word record.

The user must supply the assembly identification number and the response related identification number (IRESP). The IRESP is used throughout AKISH and JULIET to achieve correct identification and retrieval of information.

\section{1) Initiát FANISN Runs}

The initial FANISN forward and adjoint runs generate fluxes, fission densities and $k$ (if calculated). This data is stored in the repository for use by subsequent ANISN and JULIET runs. This data must be identified by setting IRESP $=1$. This identification is accomplished with the FMANG module which must be executed prior to each FANISN run. FMANG input is described in detail on pp. 6-9.

\section{2) JULIET Generalized Source Runs}

Generalized sources may be generated for several responses with a single pass through the JULIET-SOURCE execution path. Each source must be uniquely identified for correct deposit in and withdrawal from the repository. A detailed description of the JULIET-SOURCE input required to accomplish unique identification is found in chapter VII, Section B, Block 4, the 74\$s card, parameters IDA and IDF.

The user via input to the FMAVG module identifies the generalized source input to be used in the FANISN generalized flux calculation. The generalized $f 1$ ux set, $\Gamma$ or $\Gamma^{*}$, calculated by FANISN is identified by the same number used to identify the generalized source which was input to the ANISN calculation. The user must insure consistency between the response related identification numbers input to FANISN via FMANG (see $p .6$ ) and the identification numbers assigned to the generalized sources via JULIETSOURCE input (see Chapter VII, Section B, Block 4, the 74\$\$ card, parameters 
IDA and IDF).

3) Flux Preparation for Sensitivity Calculations

Any FORSS sensitivity calculation requires the preparation of at least. one of the following files.

1. $\langle\theta * \phi\rangle$ for $k$ sensitivity

2. $\left\langle r^{\star} \emptyset\right\rangle$ for rea:tion rate ratio sensitivity

3. $\left\langle r \theta^{*}\right\rangle$ and 1 and 2 for worth sensitivity

The files are prepared by the JULIET-FLUXP execution path. The user must identify the component fluxes for the sensitivity calculation. The identification of with IRESF $=1$ is assumed by the FORSS system. Any tampering with the identification of will destroy the calculation. Since is identified by default, for flux folds involving 9 , only the adjoint flux must be identified. This can be adequately accomplished with FMANG input (Section 2.A). The generation of $\langle\Gamma \emptyset *\rangle$ is more complex and correct identification requires the use of input parameters to the JULIETFuXP execution path. A detailed description of the input required may be found in Chapter VII, Section B, Block 3, the 60\$\$ card, parameters IRF and IRA.

4) Nomalization and Response Parameters for JULIET Sensitivity Calcs.

See $p$. 1 , data type 7 , for a review of normalization and response parameters. These parameters are used in the calculation of a sensitivity profile (see Chapter VII, Equations 32-37). The parameters were calculated by the JULIET-SOURCE module and placed in the repository identified with same number used to identify the adjoint generalized source (see Chapter VII, Section B, Block 4 , the $74 \$ \$$ card, the IOA parameter). Identification of these parameters to the JULIET-SENSE execution path may be accomplished with input to the rMANG module (see D. $\overline{0}$ ) which is executed immediately preceding JULIET. The identification provided by FMANG may be superseded by input to the JULIET-SENSE execution path (see Chapter VII, Section B, Block 5, 83S\$ card, IRESP parameter). 


\section{B. FORSS Manager (FMANG)}

\section{1) FANISN-JULIET Data Transfer}

FMANG manages the data transfer between FANISN and iULiET. An I/0 unit (Default $=2$ ) is used as a repository for such data. The data are categorized by three integers, assembly, response related, and type identification numbers. Normal input to FMANG is one free form card.

1\$\$ Integer Parameters [10]

1. IASB - Assembly identification number

2. IRESP - Response related identification number

3. ISIG - Status signal

0 - no useful data in repository

i - useful data in repository

4. IAC - Unit number of FANISN cross section file (Default $=4$ )

5. ITH - Enter 1 if FANISN case is adjoint

6. IQM - Number of source moments

7. 0

8. 0

9. IR2 - Unit number of the repository (Default $=2$ )

10. 0

If a zero is encountered on the $1 \$ \$$ card for a parameter that has a default value, the default value is used. Parameters 5 and 6 ITH and IQM are used in the revision of FANISN input. When zeroes are encountered on the $15 \$$ card for these parameters no data revision occurs. See the discussion of input for FANISN generalized cases in Chapter VI for more detail about ITH and IQM. When FMANG is signaled that useful data are in the repository, it scans the unit and constructs a directory of contents. This directory is used throughout FANISN and JULIET. A FMANG execution must immediately precede each FANISN and JULIET calculation in the job stream. 


\section{2) Error Recovery}

If an error is discovered which inval idates any type of data in the repository, the erroneous data mist be removed. FMAKG provides two options for describing the data to be removed.

Option I permits data description by assembly, response related, and type identification numbers.

\section{Input}

ISS Integer Parameters [10]

- NIS, the number of data types to be removed (axtered as $a$ negative number)

FO - Fill 0 option

$\mathbf{T}$

25s Integer Parameters [3*|NIS|]

IASB - Assembly ID

IRESP - Response related ID

ITYP - Data type IO

:

The 3 integer sequence is repeated NIS: times.

$\mathbf{T}$

Option 2 permits date description by block number. This option is useful if 2 or more sets of data are on the repository with identical IASB, IRESP, and ITYP identification numbers.

Input

iss Integer Parameters [10]

- NIB, the number of data blocks to be removed (entered as a negative number).

0

0

$-1$

FO

T

2\$S Integer Parameters [ $|\mathrm{NIB}|]$

IBN . . . , the block numbers to be removed 


\section{IV-10}

$?$

The following is input if an ANISN or JUL IET run is to be made after the data removal.

355 Integer Paramzters [10]

IASB - Issembly identification

IRESP - Response related identification

1 - Indicates useful data in repository

FO - Fill 0 option

$T$

3) Repository Copy

There are occasions when it is usefui to copy the repository.

Input

$1 \$ \$$ Integer Parameter [10]

82

IOUT - Unit number of new repository (default $=31$ )

IN - Unit number of existing repository (default $=2$ )

$T$

The repcsitory is copied without modification from unit IN to unit IOUT.

4) Repository List

A complete listing of the repository may be obtained or a selected

list is available with the data identified by IASB, IRESP, ITYP.

Input for complete list

$155-1100170 \mathrm{~T}$

Input for Selective List

lis Integer Parameters [10]

- NTS, the number of data types to be listed (entered as a negátive number)

1

FO 
[V-1]

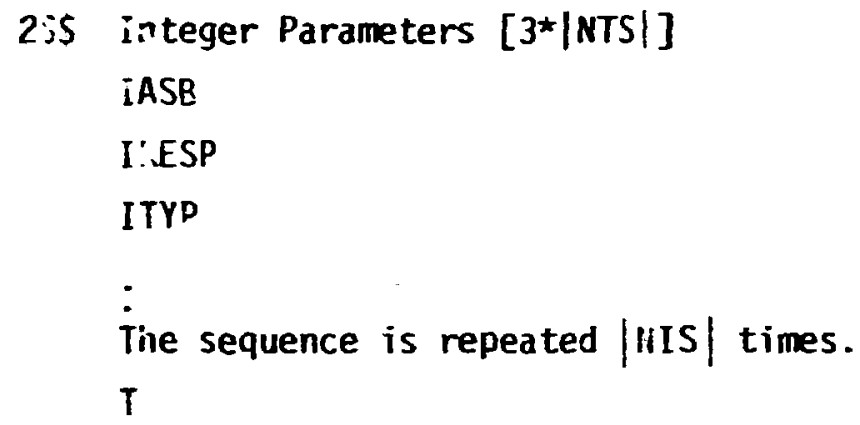


W. H. Engle Jr.

G. C. Haynes 


\section{CHAPTER V}

Table of Contents

Page No.

INPUT IRSTRUCTIORS FOR CCC-254/ANISN-ORNL ........ V-3

A. Data Description ............... v-3

B. Detailed Data Notes ........... V-. V

C. Problem Size .................. $v-18$

INPUT INSTRUCTIONS FOR PSR-75/AXMIX .......... 
INPUT INSTRUCTIONS FOR CCC-254/ARIISN-ORNL

H. H. Engle, Jr.

\section{A. Data Description}

This section is intended to be used as a guide in preparing problems for ANISN. The following section presents a more detailed description of the data. The quantity in.brackets is the array dimension and the expression in braces is the condition requiring that array or set of arrays. Arrays or sets of arrays which are not required should not be entered. If no condition is specified the array is required. Note that a $T$ must follow each of the five sets of arrays if that set is entered. (01d $A$ definition of LIMI no longer valid)

The title card may contain only 48 characters. Columns 61-72 of the title card are used to enter a meximum execution time (ignored if zero). FORMAT $(12 A 4,12 X, 112)$

Parameters

$15 \$$ Integer parameters [36]

1. ID problem ID number: if greater than $1,000,000$, disadvantage factors will be computed by group for each material which appears in the calculation

2. ITH 0 - forward solution 1 - adjoint solution

3. ISCT maximum order or scatter found in any zone ( $P$ value)

4. ISN order of angular quadrature (S value)

5. IGE 1 - slab; 2 - cylinder; 3 - sphere

6. $B \mathrm{~L}$ left boundary condition

0 - vacuum (no reflection)

1 - reflection

2 - periodic

3 - white/albedo 
7. IBR right boundary condition, same options as IBL

8. IZM number of zones or regions (same material)

9. IM number of mesh intervals

10. IEVT eigenvalue type

0 - fixed source

1 - $k$ calculation

2 - a calculation

3 - concentration search

4 - zone width search

5 - outer radius search

6 - buckling search

11. IGM number of energy groups

12. IHT position of $\sigma_{\text {total }}$ in cross section table

13. IHS position of $\sigma_{g g}$ (self-scatter) in cross section table

14. IHM length of cross section table

15. MS cross seccion mixing table length (10\$, 11\$, 12*)

16. MCR number of cross section sets to be read from cards $(14 *)$

17. MTP number of cross section sets to be read from tape (13\$)

18. MT total number of cross section sets (elements + mixtures)

19. IDFM 0 - density factors $\left(21^{*}\right)$ not used

1 - density factors used

20. IPVT 0 - no effect.

1 - enter $k_{0}$ as PV $\left(16^{*}\right)$

2 - enter $\alpha_{0}$ as PV

21. IQM 0 - no effect

1 - enter distributed source $\left(17^{*}\right)$

22. IPM 0 - no effect

1 - enter shell source by group and angle (18*)

IM - enter shell source by interval, group, and angle

23. IPP interval number which contains shell source if $I P M=1 ; 0$ otherwise

24. IIM inner iteration maximum 
25. IDI 0 - no effect

1 - print angular flux

2 - punch scalar flux

3 - both 1 and 2

26. ID2 0 - no effect

1 - use specially prepared group independent cross

section tape (contains MTP materials)

27. ID3 0 - no effect

C

$N$ - compute $N$ activities by zone where $N$ is any positive integer

28. $104 \quad 0$ - no effect

1 - compute $N$ activities by interval where $N$ refers to ID3

29. ICM outer iteration maximum

30. IDATI 0 - all data in core

1 - cross sections and fixed sources stored on tape

2 - fluxes and currents on tape also

$\checkmark 31$. IDAT2 0 - no effect

If IDAT2 is greater than zero, the first IDAT 2 outer iterations will be executed according to the specifications in the $24 \$$ array. In the $24 \$$ array 0 indicates an $S_{n}$ calculation, 1 indicates a diffusion calculation and 2 indicates an infinite homogeneous medium calculation. If convergence is not obtained after IDAT2 iterations, the problem continues using the $S_{n}$ calculation for all groups until convergence is obtained or ICM is reached.

$\checkmark$ 32. IFG -0 - no effect

1 - execute cross section weighting

2 - read cross sections weighted in previous ANISN cases

3 - write weighted cross sections on tape/disk/etc. at completion of ANISN case, or

4 - both 2 and 3

$\checkmark$ Updated 1973 
$\checkmark$ 33. IFLU 0 - step model used when linear extrapolation vio!ds negative fiux (mixed mode)

1 - use linear model only

2 - use step model only

3 - weighted difference model

4 - weighted model used as negative flux fixup for linear model

34. IFN

0 - enter fission guess (2*)

1 - enter flux guess $\left(3^{*}\right)$

2 - use fluxes from previous case

35. IPRT 0 -print cross sections

1 - do not print cross sections

$\checkmark$ 36. IXTR 0 -enter zero; not used

16* Floating point parameters [14]

1. EV first guess for eigenvalue (usually 0.0 )

2. EVM eigenvalue modifier

3. EPS epsilon - accuracy desired

4. BF buckling factor, normally 1.420892

5. DY cylinder or plane height for buckling correction

6. $0 Z$ plane depth for buckling correction

7. DFMI transverse dimension for void streaming correction

8. XNF normalization factur (issually 1)

9. PV $0.0, k_{0}$, or $a_{0}$ according to IPVT $=0,1$, or 2

10. RYF $\lambda_{2}$ relaxation factor, nomaliy 0.5

11. XLAL point flux convergence criterion if entered greater than zero

12. XLAH upper limit for $\left|? .0-\lambda_{1}\right|$ used in linear search

13. EOL eigenvalue change epsiton

14. XNPM new parameter modifier

NOTE: The above data are followed by a $T$.

$\checkmark$ Updated $19 ? 3$ 
Cross Sections $(I 02=0)$

135 Library ID numbers [MTP] (MTP >0) (Omit if using cards)

$14^{\star}$ Cross sections [MCR $\times$ IGM $\times$ IHM] (MCK $>0$ )

NOTE: If entered, the above data are followed by a $T$.

Fixed Source (IEVT $=0$ and $102<2$ )

17* Distributed source $[I G M \times I M] \quad(I Q M=1)$

18* Shell source $[I G M \times I P M \times N M]$ (IPM $>0$ )

NOTE: If entered, the above data are followed by a $T$.

Flux or Fission Guess (IFN $<2$ )

$\dot{Z}^{\star}$ Fission density [IM] $($ IFN $=0)$

$3^{*}$ Flux guess [IGM $\times$ IM] $\quad$ (IFN $=1$ )

NOTE: If entered, the above dat3 are followed by a $T$.

Rema inder of vata

1 * Fission spectrum [IGM]

$4 \dot{*} R_{i} d i i$ by interval boundary $[I M+1]$

5* :'elocities [IGM]

6* Angular quadrature weights [MM $]^{3}$

7 * Angular quadrature cosines [MM]

$8^{\star}$ Zone numbers by interval [IM]

$9 \$$ Material rumbers by zone [IZM]

los Mixture numbers in mixing table [MS] (MS > 0)

115 Component numbers in mixing table [MS] (MS >0)

$12^{\star}$ Number densities in mixing table [MS] (MS >0)

195 Order of scatter by zone [IZM] (ISCT >0)

20* Radius modifiers by zone [IZM] (IEVT $=4$ )

21 * Density factors by interval [IM] (IDFM = 1)

$22 \$$ Material numbers for activities [ID3] (ID3 >0)

235 Cross section table position for activities [I03] ( $\left.\mathrm{IO}_{3}>0\right)$

$3_{M M}=I S N+1$ for plane or sphere

$M M=(i S: ! \times(I S N+4)) / 4$ for $=y l i n d e r$ 
245 Calculation type markers [IGM] (IDAT2 = 1)

25* Albedo by group - right boundary [IBM] (IBR = 3)

26* Albedo by grojp = left boundary [I $\left.\mathrm{C}_{24}\right]$ ( IBL = 3)

275 Few group parameters [5] (IFG $=1)$.

1. ICON 0 - no effect

I - micro cross sections desired

2 - macro cross sections desired (minus impl ies cell weighting)

2. IHTF position of $\sigma_{\text {total }}$ in weighted cross sections

3. IHSF position of $\sigma_{g \rightarrow g}$ in weighted cross sections (minus implies upscatter removal)

4. IHMF table length of weighted cross sections

5. IPUN

0 - no effect

1 - punch weighted cross sections

28\$ Few group number for each multigrosp [IGM] (IFG = 1) NOTE: The above data are followed by a $T$.

"Double T"

Each ANISN/360 case must be followed by one extra card. This card may be blank or may contain the "double $T$ " if desired. If a case terminates normally, this extra card is read and ignored. If a case is terminated by an error, the "double $T$ " is searched for, and, if found, ANISN expects a title card and associated data to follow

SPECIAL NOTE: You should always put in a fission spectrum $(1 *)$ and a velocity $\left(5^{*}\right)$ array, even if they are just filled with zeros.

ANISN should always be run in a inodel which ignores floating point divide, overflow and underflow checks, and integer divide and overflow checks.

$\checkmark$ Updated 1973 


\section{B. Detailed Data Notes}

This section presents a more detailed definition of selected parameters and arrays. Some comments derived from experience are also included.

Boundary conditions - IBL, IBR, 25*, 26*

The white boundary condition causes the entering flux at the specified boundary to be isotropic. This is accomplished by summing the flux: leaving the system and returning an average flux in all directions. The albedo for each group specifies the fraction of the flux leaving to be returned. If the albedo is not specified it is assumed to be 1.0. The white boundary is recommended for the outer boundary of spherical and $c ;$ lindrical cells.

Cross section mixing table - MS, $10 \$, 11 \$, 12^{\star}$

The cross section mixing table is used to combine elements into macroscopic mixtures and to specify the method of the concentation search. Experience will reveal that only the imagination limits its flexibility. The following table illustrates the three types of operations performed by the mixing table.

$\begin{array}{cccc}\text { 1. } & \frac{105}{M} & \frac{115}{115} & \frac{12 *}{X} \\ \text { 2. } & M & 0 & X \\ \text { 3. } & M & N & 0.0\end{array}$

1. Multiply all cruss sections in material $M$ by $X$.

2. Hultiply all cross sections in material $N$ by $X$ and add to corresponding cross sections in material $M$.

3. Multiply all r.ross sections in material $M$ by EV, the eigenvalue. (concentrition search)

Cross sections - $13 \$, 14^{*}$

ANISN expects a table of cross sections for each group, 9 , of each material in the following format: 


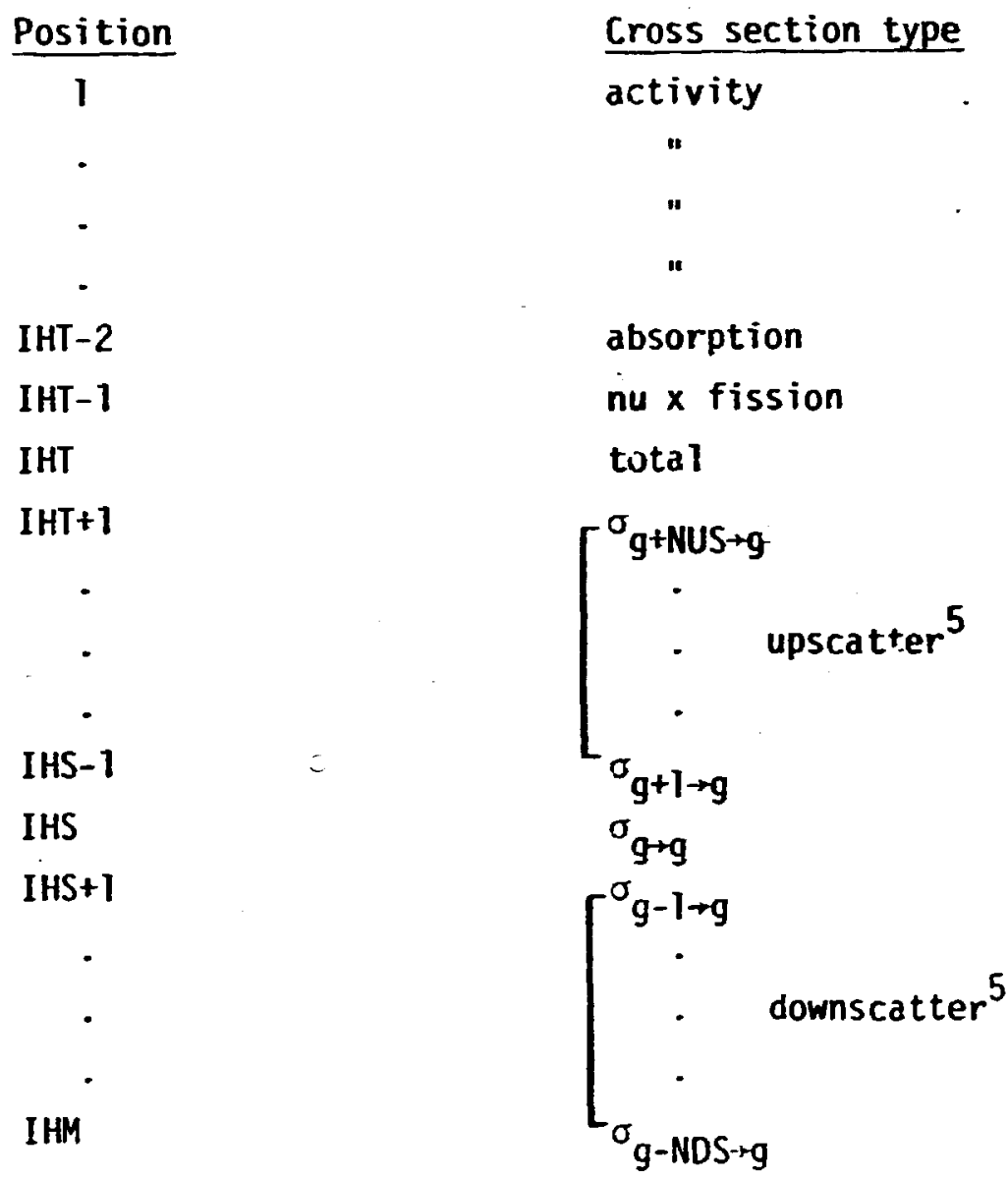

Thus, the parameters IHT, IHS, and IHM ccmpletely describe the format of the cross sections. If there are no activity cross sections, IHT $=3$. If there is no upscatter IHS $=$ IHT +1 . If there is no downscatter IHM = IHS ( $i . E .$, a one group proble... . If there is upscatter ANISN will compute a tctal upscatter cross section for each group of each material and place that cross section in position IHM +1 . The activity cross sections are used only for activities (225, 235).

The $P_{L}$ cross section tables must correspond in format to the $P_{0}$ tables even though the transfer coefficients are the only numbers used. Note that the $P_{L}$ cross sections must contain a $(2 L+1)$ term. Previous $S_{n}$ codes supplied this term internally (e.g., DTF-II multiplied by the

\footnotetext{
$5_{\text {NUS }}$ is the number of groups of upscatter NDS is the number of groups of downscatter
} 
$P_{1}$ cross sections by 3.0). This factor may be included externally or internally via the mixing table.

Material numbers - 10\$, 115, 95, 225

Al 1 cross section sets, whether elements or mixtures, are referred to by a continuous set of luterial numbers. In particular, the materials supplied in card form $\left(14^{\star}\right)$ become materials 1 through MCR, the materials read from a library tape become MCR + 1 through MCR + MTP, and any number greater than MCR + MTP but less than or equal to $M T$ refers to a mixture.

When the order of scatter for any zone (19\$) is greater than zero, ANISN expects the $P_{1}$ cross sections to be material $M+1$, the $P_{2}$ cross sections to be $M+2$, etc. where $M$ is the $P_{0}$ material number specified in the $9 \$$ array.

Density factors - IDFM, 21*

All cross sections appropriate to an interval are multiplied by the density factor for that interval. Thus, one may easily and efficientiy describe a void or a density variation by interval.

IPVT and PV - used in search

If IPVT $=1$, ANISN will search for the parameter which results in a multiplication factor of PV. If IPVT $=2$, ANISN will search for the parameter which results in a multiplication factor of 1.0 when $a=$ PV. If IPVT $=0$, ANISN will search for a multiplication factor of 1.0 with $\alpha=0.0$.

Distributed source $=1 Q M, 17^{*}$

The distributed source is entered by group and interval as follows: group 1, interval i through IM; group 2, etc.

Shell source - IPM, IPP, 18*

If IPM $=1$, the shell source is entered by group and angle for interval IPP as follows: group 1, angle 1 through angle MM; group 2, etc. 
If IPH = IM, the shell source is entered by group, interval and angle as follows: group 1 , interval 1, angle 1 through angle NM; interval 2 , etc.

Special cross section tape - $182=1$

A special purpose program is avaitable which will prepare a group independent cross section tape for ANISN. This tape is required if the complete input cross section matrix [(MCR + MTP) $\times$ IGM $\times$ IHM] is larger than the number of data locations available.

Activities - ID3, ID4, 22\$, 235

Activities may be computed by zone and interval as specified in 103 and ID4. The zone activity is a total reaction rate and the interval activity is per unit volume. The following table illustrates the use of activity specifications.

$\begin{array}{ccc}\text { 1. } & \frac{225}{1} & \frac{235}{3} \\ \text { 2. } & -5 & ? \\ \text { 3. } & 7 & -1 \\ \text { 4. } & -3 & -1\end{array}$

1. Compute activity for material 1, crcss sfiction position 3 in the intervals and/or zones in which material 1 appears.

2. Compute activity for material 5, cross section position 1 in all intervals and/or zones.

3. Compute activity for material 7, position 1 in appropriate intervals and/or zones and multiply interval activities by $1.0,2-r$, or $4-r^{2}$ for slab, cylinder, or sphere respectively.

4. Compute activity for material 3, position 1 in all intervals and/or zones and multiply interval activities by geometry factor.

Auxiliary tape storage - IDATI

If IDATI is specified as zero, ANISN will use the most efficient tape storage possible and modify [DAT] accordingly. IUATI may be specified as 1 or 2 if cross section and/or flux tapes are available from a previous problem or if ID2 $=1$. 
Diffusion theory solution or infinite homogeneous medium solution IDAT2, 245

If IDAT2 $=1$ the 245 array must be entered. A zero implies a transport solutior, a one implies a diffusion solution, and a two implies an infinite medium solution for the corresponding group. If IFN $=0$, ANISH will use diffusion theory on the first outer iteration.

Weighted cross sections - IFG, $275,285^{\star}$ (see bottom of page)

When microscopic weighted cross sections are requested (ICON $=1$ ), a set of cross sections is produced for each component of each material in each zone. When nacroscopic cross sections are requested (ICON $=2$ ), a set of cross sections is produced for each material in each zone. The cross sections are weighted by the flux or current in the zone in which the material appears. Since the mixing table is used to determine the components of a material, MS should not be zero when ICON $=1$.

If the cross section structure specified for the weighted cross sections will not accommodate the complete multigroup scattering matrix, the "extra" transfer coefficients are placed such trat they transfer as far down (or up) as possible.

If comriete removal of the upscatter is desired, IHSF should be minus. |IHSF! should be the position of the sel? scatter cross section before the upscatter is removed. IHMF should be the final table length. After the upscatter is remuved, IHSF will be IHTF +1 . The upscatter is removed by subtracting the reaction rate due to $\sigma_{j \rightarrow j}$ from the reaction rate due to $\sigma_{i \rightarrow j}$ where $j>i$. Thus, the net transfer rate between groups $j$ and $i$ is preserved.

"When IFG is 1,3 , or 4 the $27 \$$ and $28 \$$ arrays must be entered. When IFG is 2 or 4 , cross section order is as follows: 'materials 1 through $M$ weighted by previous case; materials $M+1$ through $M C R$ - read from cards; materials $M C R+1$ through $M T P+M C R$ - read from library; materials $M C R+M T P+1$ through MT - mixtures. 
Starting guess - IFN, 2*, $3^{\star}$

If IFN is specified as zero, ANISN will execute a diffusion solution for the first outer iteration. Since this is undesirable for fixed source calculations where one normally desires a zero flux suess one may set $I F N=1$ and enter no guess. Simply enter a card with a $T$ in column three for that section of data.

EV and EVM guesses

\begin{tabular}{crc} 
IEVT & EV & EVM \\
\hline 0 & 0.0 & 0.0 \\
1 & 0.0 & 0.9 \\
2 & best guess for $\alpha^{6}$ & 0.0 \\
3 & 1.0 & -0.1 \\
4 & 0.0 & -0.1 \\
5 & outer radius & $-\left(10^{\circ}:\right.$ of outer radius $)$ \\
6 & 1.0 & -0.1
\end{tabular}

When IEVT $=0$ there is no eigenvalue (EV).

When IEVT $=1$ the multiplication factor $(k)$ is the eigenvalie.

When IEVT $=2 \alpha$ is the eigenvalue.

When IEVT $=3$ the eigenvalue is defined by its use in the mixing table.

When IEVT $=4$ the eigenvalue is used as follows:

$\Delta R_{I}=\Delta R_{I}^{0}\left(1.0+E V \times R M_{Z}\right)$

where $\Delta R_{I}^{0}$ is the initial $\Delta R$

$\mathrm{RM}_{Z}$ is the radius modifier $\left(20^{*}\right)$

When $I E V T=5$ the suter radius is the eigenvalue.

When IEVT $=6, E V=D Y / D Y^{\circ}=D Z / D L^{\circ}$

where $D Y^{\circ}$ and $D Z^{\circ}$ are input.

Convergence - EPS, XLAL, RYF

The inner or flux iterations are considered converged when bcth

Zero is the best guess unless one is reasonably sire that his guess is close to the answer. 
the integral self-scatter error and the integral removal error are less thin EPG or when the maximum flux deviation is less than EPS. EFG is related to EPS by a normalization factor, the total source divices by IGM. Since the integral tests are sometimes easily satisfied, a point flux conve gence may be specified. If XLAL is greater than zero, the inner iterations are not considered converged until the maximum flux deviation is less than XLAL.

The outer or power iteration is considered converged when the total source ratio between successive iterations differs from 1.0 by less than EPS, the total scatter ratio differs from $\mathbf{i} .0$ by less than EPS/RYF and the upscatter ratio differs froin 1.0 by less than EPS/RYF.

Buckling correc ion - BF, DY, DZ

ANISN computes a correction factor of the $O B^{2}$ form for finite transverse dimensions. Tre correction is applicable only with "transport currected" $P_{0}$ cross sections where position IHT is occurjied by the transport cross section.

Void streaming correction - DFMI

Since the $D B^{2}$ term is not applicable to a void region, ANISN computes a simple correcticn ${ }^{7}$ which effectively removes the transverse component of eacn angular flux in the void region. This correction term is not included in the calculation of the absorption reaction rate as are the $D B^{2}$ losses. This omission causes the neutron balance to differ from 1.0. If DFMI is zero, no correction is computed for the void regions. Normalization

When IEVT is greater than zero the total fission source is normai ized to XNF. When IEVT is equal to zero the tucal fixed source is normalized to XNF and the fission source, if any, is unnormalized. If $X N F=0.0$ there is no normalization.

Olsen, T., "Void Streaming in $S_{n}$ Calculations," Nuc?. Sci. Fng. 21
271 (1965). 
Searches - XLAH, ZQL, XNPM

When the absolute value of the difference between two successive lambdas $\left(\lambda_{1}\right)$ is less than $E Q L$, the eigenvalue, EV, is changed. The first EV chans? is the result of adding or subtracting the eigenvalue modifier, EVM. The second EV change is the result of a linear extrapolation. To prevent large changes early in the calculation, the absolute value of the oifference between 1.0 and $\lambda_{1}$ is not allowed to exceed XLAH. To prevent oscillations when using the linear search, the extrapolation is 1 imited by XNPM. The third EV change is the result of the quairatic search. The quadratic search is used until the absolute value of $1.0-\lambda_{1}$ is less than $E Q L$. At this point, the linear search is used to complete the problem. XLAH is normally 0.05 and XNPM is normally 0.75 . EQL should be the larger of $r . \overline{0} 01$ and three times EPS. In cases where. EPS is quite small, EQL may be less.

\section{Multiple cases}

The ANISN data arrays are stored in core in the order in which they are numbered. For example the fission density $\left(2^{*}\right)$ follows tre fission spectrum $\left(1^{*}\right)$. No data are destroyed between cases. If prutiem dimensions change, the repositioned arrays are simply read into core over the previous data. The result is that all errays following and including the first array tr, be repositioned must be respecified. It should be noted that the $15 \$$ and $16^{*}$ parameter arrays are exceptions to the above discussion and are never destroyed. Multiple cases in which IOATl changes will not retain data properiy. If IDATl $=1$, multiple cases will retain data prcperly only if $152=2$ in all cases following case 1 . If IOATI = 2 , multiple cases will retain data properly only if ID2 $=2$ and IFN $=2$ in all cases following caje 1. If there is upscatter, multiple cases will retain data properly only if ID? = 2 or if the complete cross section matrix is read in all cases.

In cases where the data in a particular section remains the same in multiple cases, one may enter a card containing only a $T$ in any of the six appropriate columns for that section of data (e.g., the 155, 16* section). 
If any case is preceded by an adjoint solution, the following arrays must be respecified if they are required for the next case: $14^{\star}, 17^{\star}$, $18^{\star}, 3^{\star}, 1^{\star}, 5^{\star}, 24 \$, 25^{\star}, 26^{\star}$.

If multiple cases are completely independent of each other (i.e., all data are specified in each case) the data of each case may be terminated with a $T$ in the third column of two successive fields on the same card. This "double $T$ " is used in lieu of the normal single $T$. If ANISN terminates a case for any reason, the code will search for the "double T" and attempt to execute the following problem. This option may also be used for independent sets of multiple cases. 
c. Problem Size

\begin{tabular}{|c|c|c|c|}
\hline$(24)$ & {$[240]$} & $($ IM) (IDFM) & {$[0]$} \\
\hline$(I G M+1)(2)$ & [8] & $(I M)($ IGM $)$ if IDATI $<2$ & {$[105]$} \\
\hline$(\operatorname{IGM})(5)$ & {$[15]$} & (IM) if IDATI $=2$ & {$[0]$} \\
\hline$(I M ! ! I 1)$ & {$[385]$} & $(I H P)(I G M)(M T)^{8}$ if IOSTI $=0$ & {$[216]$} \\
\hline$(I M+1)(4)$ & {$[144]$} & $($ IHP $)(M T)$ if IDATI $>0$ & {$[0]$} \\
\hline$(\operatorname{MH})(5)$ & {$[25]$} & $(I M)(I G M)(I Q M)$ if IDATI $=0$ & {$[0]$} \\
\hline$(I Z M)(3)$ & {$[9]$} & $(I M)(I Q M)$ if IOATI $>0$ & {$[0]$} \\
\hline$(M S)(3)$ & {$[36]$} & $(I P M)(M M)(I G M)$ if IDATI $=0$ & {$[0]$} \\
\hline (MTP) & {$[0]$} & $(I P M)(M M)$ if IDATI $>0$ & {$[0]$} \\
\hline$(103)(2)$ & {$[12]$} & (IGM) if IBR $=3$ & {$[0]$} \\
\hline$(\mathrm{IFG})(5)$ & {$[5]$} & $(I G M)$ if $I B L=3$ & {$[0]$} \\
\hline$($ IFG $)($ IGM) & {$[3]$} & $(M M)(\mathrm{JT})$ if $\mathrm{ISCT}>0$ & {$[15]$} \\
\hline$(\mathrm{IM})(\mathrm{ISCT})$ & {$[105]$} & $\begin{array}{l}(\mathrm{IM})(\mathrm{JT})(\mathrm{IGM}) \text { if } \mathrm{ISCT}>0 \text { and } \\
\text { IDATI }<2\end{array}$ & {$[315]$} \\
\hline \multicolumn{4}{|c|}{$(I M)(M M) \quad(1973)$} \\
\hline$(I M+1)(N M)(5)$ & {$[900]$} & $(\mathrm{IM})(\mathrm{JT})$ if ISCT $>0$ and IDATI $=2$ & {$[0]$} \\
\hline$(\mathrm{IM})(\mathrm{JT})(2)$ & {$[210]$} & $(M M)(I G M)$ if IDATI $<2$ & {$[15]$} \\
\hline$(I M)(J T)+1$ & {$[106]$} & (MM) if IDAT! $=2$ & {$[0]$} \\
\hline (IGM) (IDAT2) & {$[0]$} & TOTAL & {$\left[2869^{\circ}\right.$} \\
\hline
\end{tabular}

To determine the number of data locations required for a given problem, each of the above expressions should be evaluated and sumed. The numbers in the brackets apply to the sample problem.

\footnotetext{
8 IHP $=$ IHM if there is no upscatter

$I H P=I H M+1$ if there is upscatter
} 


\section{INPUT INSTRUCTIONS FOR PSR-75/AXMIX}

G. C. Haynes

TITLE CARD -- First card of a problem, Format (20.44), identifying the problem and/or the output data set.

AXMIX Control Parameters (Array BTock 1)

IS Array -- Primary Control Parameters (12 entries)

1. IGM

Number of energy groups for this problem

2. ITLI

Input $x$-sect table length

3. ITLO

Output $x$-sect table length. If ITL $\theta \neq I T L I$, the difference is either truncated or padded with zeros. (At the end of the table if ITLD is positive, or at the beginning if ITLO is negative).

4. $M C R$ Number of materials inpst from cards $\left(30^{\star}\right)$. A "material" is one IGM*ITLI cross-section set.

5. MTP Number of materials input from nuclide libraries (135).

6. MGIP

Number of materials input from GIP data sets (15\$). $+M$ means entire GIP input is on KUl. -M means GIP input is specified in $15 \$$ array.

7. NOS13 Number of nuclide libraries specified by lagical unit number (negative ent $j^{\prime}$ ) in the $13 \$$ array. If NOSI $3=0$, MTP materials are input from unit KU3.

8. NOS 15 Number of GIP data sets specified by logical unit number (negative entry) in the $15 \$$ array. If NOS15+0, MGIP materials are input from unit KUI.

9. MS $x$-sect mixing table length $\left(10 \$, 11 \$, 12^{\star}\right)$.

10. $M T$

Total number of materials processed in this problem. $M T=M C R+M T P+M G I P+m i x$ tures. All materials are numbered consecutively from 1 through $M T$, and are defined in the order of the terms of the equation above. 
11. ITPOUT

Output nuclide library control (16\$), such that:

0 means no output 1 ibrary, omit $16 \$$ array.

1 means output materials according to $16 \$$ array onto unit KU4.

2 or $\mathbf{N}$ means combine materials according tc $16 \$$ with materials on unit KCMB and write all onto unit KU4. $N=$ (table length) $*$ (no. of groups) for largest material on KCMB if greater than IGM*ITLD.

(Check IDLST (3\$ array) when using this option)

12. IDUP4

0 means no effect.

$K$ means make extra copy of KLi4 onto unit $K$.

$-K$ means same as $K$, but prevents rewind at completion of this problem.

7 means copy KU4 onto unit 7 in fixed FIDO card image format.

-7 mearis copy KU4 onto unit 7 in free FIDO card image format.

When using unit 7 in this option be sure to specify PARM. GO=' $\mathrm{CK}=-7$ ' because unit 7 will be rewound at problem completion.

25 Array -- Control for GIP data sets, $P_{n}$ Adjustment

1. IGIPD

Output GIP data set control (17\$) such that:

0 means no output GIP data set, omit 175 array.

1 means wrice materials as specified in the

$17 \$$ array onto GIP unit KU2.

- 1 means same as 1 except output GIP data

set is adjoint. (If this option is used, ru input GIP data set is allowed, and all output will be adjoint.) 
Note: If a GIP tape is to be output, cross sections may not contain upscatter. This limitation does not apply to input GIP data sets. (See special section on upscatter)

2. IPNC

0 means no $P_{n}$ adjustment, omit 215 through 245 arrays.

$J$ means do $P_{n}$ adjustments on $J$ elements or mixtures as specified in the 215,225 , $23 \$$, and $24 \$$ arrays. (In these arrays refer to only the $P_{0}$ component of each element or material to be adjusted.)

3. IHT

4. IHS

5. ITRC

5. IHTSAV

7. IADI
Table position of total cross section.

( lot used if IGIPG $=I P N C=I T R C=0$ )

Table position of within-group scattering cross section.

(Not used if IGIPD $=I P N C=I T R C=0$ )

Note: If IHS > IHT +1 , upscattering is implied and any output GIP libary requested will be aborted.

Output logical unit for a transport-corrected cross section library to be written according to the 255 ID array. Negative entry prevents rewind at problem completion. Cross section table position to sove sigma total in the transport library if IiPr $\neq 0$, (zero means no effect) Upscatter GIP data set input type. 0 means input data sets contain no upscatter (be sure IHS=IHT+l). 1 means input data sets were for ANISN, and the total upscatter coefficients are in table position ITLI+1. 
2 means input data sets were for DOT, and the total upscatter coefficients are in table position IHT +1 .

Note: GIP data sets contain one extra table position for total upscatter coefficient. Disregard this extension of the table length when specifying ITLI.

8. LADO Not presently used. Enter zero.

This array defaults to all zero values if not entered as input. 35 Array -- Input/Output Logical Units (8 entries, default values in parenthesis)

1. KUI

2. KU2

3. KU3

4. KU4

j. KU5

6. KU6

7. $\mathrm{KCMB}$
11) GIP tape input logical unit number. This number is used if the 155 array is not entered. or if the first entry in the 155 array is positive. Negative entry prevents rewind at input completion.

(2) GIP tape output logical unit number. Negative entry prevents rewind at problem completion.

(3) AldISN/COT nuclide library input logical unit number. This number is used if the first entry in the 135 array is positive.

(4) ANISN/OOT nuclide library output logical unit number. Negative :ntry prevents rewind at probiem completion.

(5) Card input logical unit number.

(6) Printed output logical unit number.

(8) Logical unit number for old ANISN/DOT nuclide library to be combined with new library. The resulting library is written on unit kU4 and the library on $K C M B$ is unaltered if KCMB $\neq K \cup 4$ 
8. IDLST

$$
\begin{aligned}
0= & \text { (if ITPDUT<2) No effect. } \\
0= & \text { (if ITPDUT=2) Merge output with KCMB, ID } \\
& \text { numbers in ascending order (KCMB and KU4 } \\
& \text { must be different); KCMB is not altered. } \\
-I= & \text { Same as above = but drop I materials (145) } \\
& \text { from library on KCMB when transferring to } \\
& \text { KU4. } \\
I 0= & \text { Add output to KU4 following this ID on KCMB } \\
& \text { (KCMB may eaual KU4). }
\end{aligned}
$$

This array is not required if the default values enclosed in parentheses are satisfactory. Any units not required by a problem are irrelevant, no DD cards are required for them, and any integer will suffice for them.

Puncned output, if any, nust be output to logical unit number 7 .

Note: The 15, 25, and 35 arrays are the first array block for a probleri, and the block is terminated with a " $T$ ".

AXMIX Input Arrays (Array Block 2)

lOS Mixture numbers in mixing table. [MS entries] Negative entry causes division $\left(10 \$ /\left(115^{\circ} 12^{\star}\right)\right)$

IIs Component numbers in mixing table [MS entries] Negative entry causes multiplication $\left(105 \cdot 115 \cdot 12^{\star}\right)$

12* Number densities in mixing table [Ms entries]
[M

135 Input ANISN/DOT nuclide library ID numbers, and logical unit numbers if NUS13>0. Data set logical unit numbers, if any are negative entries which are followed by the 10 numbers of materials required from that data set. Duplicate ID's are permitted if they appear on different data sets. Entries may be up to 8 digits long. If the first entry is positive, it is assumed that KU3 is the data set logical unit number for all ID's before the first negative entry. [MTP+NDSI3 entries]

145. List of 10 numbers of materials to be dropped from library on KCMB when merging output with KCMB and [-IDLST entries] writing on KU4. $\quad$ \{omit if $10 L S T \geq 0$ \}

15\$ Input GIP tape control array. Data set logical unit numbers, if any, are negative entries which are followed by the pnsition 
numbers of materials required from each data set. Duplicate position numbers are permitted if they occur on different data sets. If the first entry is positive, it is assumed that IUI is the data set logical unit number for all entries before the first negative entry. Poisition numbers need not satisfy any fixed order.

[-MGIP+NDS15 entries] \{omit if MGIP>0\}

165 Outfut ANISN/DOT ruclide library ID numbers. These may be up to 8 digits long (o digit limit is recomended), and need not be arranged in order. One entry is required for each material of the problem, and a zero means that materia: is not output. If this library is being merged with one on KCMB, a new material may be substituted for one on KCMB by using the same ID number. Regardless of the array order here, all resulting output is witten in order of ascending IO number. [MT entries]

\{omit if ITPQUT $=0$ \}

175 Output GIP tape control array. One entry is required for each material of the problem. A zero entry means that material is net output. Any nonzero entry (usually a 1) means that material is output.

[MT entries] iomit if IGIPQ $=0$ ?

185 Output cross-section title control array, one entry for each material.

A zero entry means no title is to be assigned to that material. Any cositive entry means keep cld title if one exists (from ar. ANISN/DOT nuclide library, KU3, not KCME).

Any negative entry means a new title is to be read from a title card following the last data block of that problem.

[MT entries]

\{omit it all pos:

195 Output cross-section print control array, one entry for each material.

A zero entry means do not print that material. 
Any nonzero entry means print that material.

[RT entries]

comit if all zero\}

LOS Output cross-section punch control array, one entry for each material.

A zero entry means do lot punch that material.

A positive entry means punch in fixed FIDP format.

A negative entry means punch in free-form FID format.

215 Material numbers of the $P_{9}$ scattering components of elements or mixtures to be " $p_{n}$ adjusted". [IPNC entries]

225 Material number of $P_{0}$ component to be used for the $P_{n}$ adjustment.

[IPNC entries]

235 New (adjusted) scattering order of elements or mixtures

identified in the 215 array. [IPNC entries]

245 Energy groups to which the $P_{n}$ adjustment is to be applied, one entry for each group.

4 zero menas that group is not affected.

Any nonzero entry means that group is affected.

[IGM entries]

\{omit if IPNC=0\}

2555 Output ID numbers for $P_{0}$ materials to be "transport corrected". Zero entry for all other materials. Be sure-the $P_{1}$ material follows the $P_{0}$ material referenced here.

[MT entries]

\{omit if ITRC $=0$ \}

NOTE: End of array block 2, terminate with " $T$ "

Card Input Cross-Sections (Array Block 3)

$30^{*}$ and/or 30** and/or any number from 01 through 99

All cross sections to be input from cards go here, one material immediately following another. They may be fixed FIDO format and/or free FIDO format. If the format changes, however, a new $30^{*}$ or $30^{* *}$ must be entered to indicate the format of the data following it.

[MCR*(IGM*ITLI) entries]

NOTE: This is the final array block, and it must be terminated with a "T". 
(Caution: The " $T$ " must not be immediately preceded by a " $F "$

Option) Omit entire block, including the "T" if MCR=0

\section{Cross-Section Title Cards}

A title card must be entered, following the last array block, for each negative entry in the $18 \$$ array. Any desired title is punched in Columns 25-72 of each card, and all other columns are irrelevant.

\section{Multiple Problems}

Multiple problems may be run consecutively by following the input to a previous problem with a new problem title card and new input data. Data from the previous problem is not automatically saved, and all default options apply independently to each problem. Output data sets from a previous problem, however, may be referenced as input to a problem if they were rewound. Data sets from previous problems may be added onto if they were not rewound.

Job Termination

A job may be terminated by any of the following ways:

1. End of data set on unit 5.

2. "STOP" command by entering a final card with STOP in Colurins $1-4$.

3. Call to "EXIT" by entering a rinal card with EXIT in Columns $1-4$. 



\section{CHAPTER VI \\ Table of Contents}

Page No.

FORSS ANISN (FANISN) Generalized Flux .......... VI-3

FANISN Input for Generalized Calculations . . . . . . . VI-4

Sample Input for FANISN Generalized Case ......... VI-5 


\section{FORSS ANISN (FANISN) GENERALIZED FLUX}

The heoretical development for FANISN generalized flux calculations may be found in ORNL/TM-4437 by E. M. Oblow. The calculation of sensi-

vity coefficients for linear responses such as reaction rate ratios and bilinear responses such as worth resuire the use of generalized fluxiss. Tho linear response uses a generalized adjoint flux $\Gamma^{*}$ and the bilinear res conse uses $r^{\star}$ and in addition a generalized forward flux $r$. These generalized fluxes are calculated by FANISN as a function of generalized sources winich are calculated by the JULIET mudule (see rhapter vII). This dictates the following hierarchy of events which must precede the calculation of generalized fluxes.

1. The preparation of ANISN multigriup cross sections.

2. The calculation of ANISH forward Tlux, $\psi$.

3. The calculation of ANISN adjoint flux, $\phi^{*}$.

4. For li iear responses, the ralculation of a linear source for each response.

5. For bilinear responses, the calculation of a bilinear forward and adjoint source for each response.

The results of the calculations performed in steps 2 through 5 are placed in the repository (see Chapter IV) and the calculaiion of yeneralized fluxes can now proceei. :.j demonstrated by steps 4 and 5 , generalized fluxes are response dependent and the necessary care must be taken to insure that a gereralize: flux calculated for a particular response is not used in subsequent calculations for alij ucher response. The definition of the IRF.SP parameter in the FMANG input (see chapter IV) deserves carefül attention. The system cannot detect response identification errors and if such errors are made disaster is certain and often subtle. 


\section{$\ddot{v} \bar{I}-\dot{4}$}

The following modifications to the input data for the FANISN forward calculation which generated $\$$ results in the input required for an FANISN generalized cal:ulation.

\section{FANISN INPUT FOR GENERALIZED CALCULATIONS}

The input for the initial ANISN forward $k$ calculation is defined as the reference case.

= FMANG

$1 \$ \$$ IASB IRESP 1 FO T

IASB - Assembly identification number

IRESP - Is the response related identification number. It must be set to the appropriate source identification number specified in JULIET.

Changes to the $15 \$ \$$ card of the reference ANISN case.

Parameter 2 ITH $=1$ if adjoint

Parameter 10 IEV $=0$

Parameter 20 IPVT $=1$

Parameter $21 \mathrm{IQM}=1$ for 1 inear source

$$
I Q M=\text { number of moments of } P_{l} \text { source }
$$

Parameter 33 IFLU $=1$

Parameter 34 IFN $=1$

Changes to the $16^{\star \star}$ card of the reference ANISN case.

Parameter $1 \mathrm{EV}=0.0$

Parameter 2 EVM $=0.0$

Parameter $9 P V=k_{\text {eff }}$

Remove the $2 * \star$ fission density data, replace with $3^{\star \star}$ F0.0 T. 
FMANG (see Chapter IV) and FANISN can revise the input for a preceding FANISN forward case and automatically generate the input required for a generalized FANISN case. This is demonstrated in a comprehensive sample case at the end of Chapter VII.

C SAMPLE INPIT FOR FANISN GENERALIZED CASE

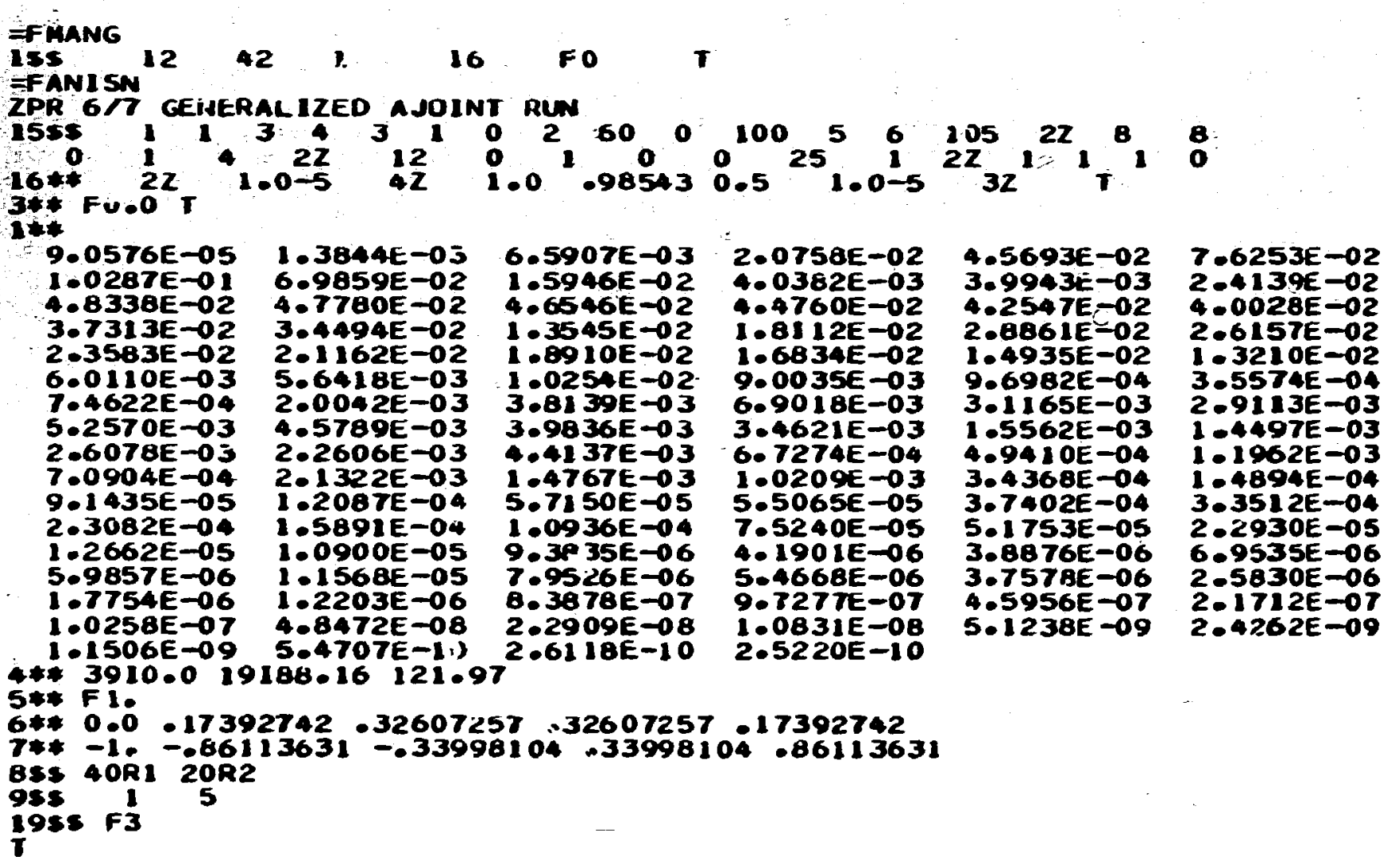




\title{
$c$ \\ CHAPTER VII. A USER'S GUIDE FOR THE JULIET MODULE OF THE FORSS SENSITIVITY AND UNCERTAINTY ANALYSIS CODE SYSTEM
}

\author{
J. L. Lucius \\ E. M. Oblow \\ G. W. Cunningham, III
}

* originally published as ORNL/TM-6594 


\title{
Contract :00.:-7405-eng-26 \\ Engineering Physics Division
}

A USER'S GUIDE FOP. THE JULIET MODJLE

OF : THE FORSS SENSITIVITY AND UNCERTAINTY ARALYSIS CODÉ SYSTEI

J. L. Lucius*

E. H. Obiow

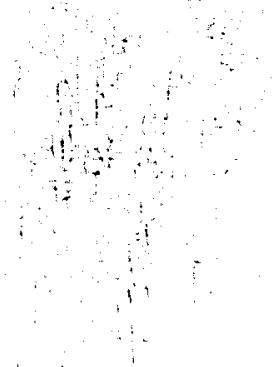

\author{
G. W. Cunningham, $I I I^{\star}$ \\ ate Published: February 1975
}

NOTICE This documenc contains information of prel iminary nature. It is subject to revision or correction and therefore does not represent. a final report.

*Computer Sciences Division

OAK RIDGE NATIONAL LABORATORY

Oak Ridge, Tennessee 37830

operated by

IINION CARBIOE CORPORATION

for the

DEPARTIIENT OF ENERGY 
TABLE OF CONTENTS

Page

ABSTRACT $\ldots . \ldots \ldots \ldots \ldots \ldots$

1 INTRODUCTION . . . . . . . . . . . . . . . VII-3

II EXECUTION PATHS . . . . . . . . . . . . . . . VII-5

A. Operator Definition .............. VII-5

= HiuxPreparation .............. VII-5

C. Source Response Calculation ........... VII-5

D. Sensitivity Coefficient Generation ......... VII-6

III. OPERATOR DEFINITION . . . . . . . . . . . . VII-6

IV. FlUX PREPARATION .................. VII-7

A. Moment Fluxes ................ VII-7

B. $\left\langle\phi^{\star} \phi\right\rangle$ Files .................. VII -8

y. SOURCE RESPONSE ALGORITHMS ............... VII-9

A. The $\left[\mathrm{H}_{1} \phi\right]$ and $\left[\mathrm{H}_{2} \phi\right]$ Components of a Bilinear Response VII-9

1. Total ................... VII-G

2. Scattering for Slab and Spherical Geometrics... VII-10

3. Scattering for Cylindrical Geometry ....... VII-10

4. Fission .................. VII-11

5. The $\left[\mathrm{H}_{1} \phi\right]$ Term ............. VII-1I

6. $\left[\mathrm{H}_{2} \phi\right]$ is a fission function with one term ... VII-11

B. The $\left[H_{1}^{\star} \phi^{\star}\right]$ and $\left[H_{2}^{\star} \phi^{\star}\right]$ Components of a Bilinear

Response................. VII-II

1. Total ....................... $\mid[\lfloor[-1]$

2. Scattering.............. VII-12

3. Fission .................... VII-12 
TABLE OF CONTENTS (Continued)

Page

4. The $\left[H_{1}^{\star} \phi^{\star}\right]$ Term $\ldots \ldots \ldots$ VII 12

5. The $\left[H_{1}^{*} \phi^{*}\right]$ function if very similar to the $\left[H_{1} \phi\right]$ function ................ VIll-12

c. The Bilinear Response ........... VII-12

D. The Bilinear Forward Source ......... VII-13

E. The Bilinear Adjoint Source ......... VII-13

F. The Worth in an Interval .......... VII-13

G. The Linear Response ............ VII-13

H. The Linear Source . . . . . . . . . VII-14

VI. GENERATION OF SENSITIVITY COEFFICIENTS ....... VII-14

A. General Representation of Terms in Sensitivity Coefficient Definitions .......... VIIL-14

B. K Sensitivity ................... VII-16

1. Absorption Reactions, Nonfissionalble, Nonscattering Reactions such as Capture ... VII-16

2. Fission Reactions ............ VII-16

3. $v$ Sensitivity .............. VII-16

4. Scattering Reactions ........... VII-16

5. Total Reaction of a Fissionable Material ... VII-17

6. Total Reaction of a Nonfissionable Material . . VII-17

c. Reaction Rate Ratios ............. VII-17

1. indirect Effect ............. VII-17

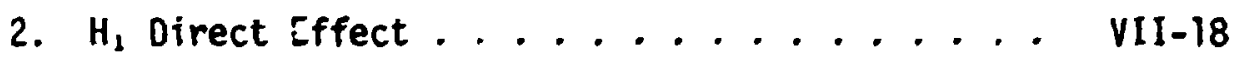

3. $\mathrm{H}_{2}$ Direct Effect ................. VII-18 
TABLE OF CONTENTS (Continued)

Page

D. Worth Sensitivities ............... VII-19

1. $H_{1}$ Direct Effect ............ VII-19

2. $\mathrm{H}_{2}$ Direct Effect ............. VII-19

3. Eigenvalue Indirect Effect ......... VII-19

4. Forward Flux Effect ............ VII-19

5. Adjoint Flux Effect ............. VII-19

VII. JULIET INPUT PREPARATION . . . . . . . . VII-2I

A. Execution Paths $\ldots \ldots \ldots \ldots \ldots$ yl1-21

1. Operator Definition, Selected by the Word, MIX . VII-22

2. Flux Preparation, Selected by the Hord, FLUX . . VII-22

3. Source Generation, Selected by the Word, SOURCE . VII-22

4. Sensitivity Coefficient Generation, Selected by the Word, SENSE .......... VII-23

B. Input Blocks .................. VII-23

1. Clock 1 (always provided) .......... VIl-23

2. Block 2 (aiways provided) Uperator Definition .. VII-25

3. Block 3 (Optional) .............. VII-28

4. Bloc': 4 (Optional) ........... VIII-28

5. Block 5 (Optional) ............ VII-30

VIII. RESOURCE IJTILIZATION ..................... VII-34

A. Eontainer Array ................ VII-34

1. Operator Dafinition ............. VII-35

2. Flux freparation ............. VII 35

3, Source Response . . . . . . . . . VII-35

4. Sensitivities . ........... VII-36 
TABLE OF CONTENTS (Continued)

Page

c

B. Overlay Structure ................ viII -36

C. I/0 Units .................. VII -38

IX. JULIET ERROR STOPS . . . . . . . . . . . VII -41

A. The Unnumbered STOP Statements . . . . . . . VII -4I

B. The Numbered STOP Statements ......... VIII-42

C. Subroutine ERRA ................... VIII-43

D. Subroutine CRASH $\ldots \ldots \ldots \ldots \ldots$ VII -44

X. THE MATS CROSS-SECTION FILE ........... VII-45

XI. SAMPLE PROBLEM ................ VII -48

ACKNOWLEDGMENTS $\ldots \ldots \ldots \ldots \ldots \ldots$ VII -54

REFERENCES $\ldots \ldots \ldots \ldots \ldots \ldots \ldots \ldots \ldots$ VII -55

vi t 


\section{VII -3}

A USER'S GUIDE FOR THE JULIET MODULE OF THE FORSS SENSITIVITY AND UNCERTAINTY ARALYSIS CODE SYSTEA

J. L. Lucius, E. M. Oblow, and G. W. Cunningham, III

\section{ABSTRACT}

JULIET is the FORSS module that calculates generalized sources, responses (e.g., criticality, reaction rate ratios, reactivity worths), normalization parameters and sensitivity coefficients. JULIET is organized into execution paths which are in effect submodules. This permits a problem to be segmented for solution at the user's discretion (i.e., multiple entry points). JULIET nomally operates with flixes generated by the FORSS version of ANISN; however, the execution path concept permits interaction with other neutronics codes such as DOT and VENTURE.

The proposed CCCC file MATXS is the cross-section data base for JULIET permitting the calculation of sensitivity coefficients with respect to partial cross sections. The sensitivity coefficients calculated by JULIET are placed in the proposed CCCC file SENPRO where they may be accessed by other modules in the FORSS system or transmitted to other installations.

\section{INTRODUCTION}

JULIET is the FORSS module that calculates sources, responses (e.g., criticality, reaction rate ratios, reactivity worths), normalization parameters, and sensitivity coefficients. As a user', guide, this document presents the aigorithms implemented in JULIET but does not include their theoretical development which is available in Ref. 2. JULIET optimizes and extends algorithms that were initially implemented in the SOURCE (see Ref. 2 for theoretical development) and SWANLAKL ${ }^{3}$ codes. JULIET is designed to execute as a module of the FORSS driver controlled system. It withdraws and deposits information in the FORSS repository which serves as an information exchange medium for FAliIS: i $^{14}$ (the FORSS 
version of ANISN) and JULIET. When FANISN has been selected as the neutronics code for a FORSS study, JULIET receives angular fluxes and computes generalized source data for subsequent steps in which FANISH is used to generate generalized ${ }^{2}$ angular fluxes. JULIET is organized into execution paths which are in effect subnodules. This permits a problem to be segmented for solution at the user's discretion (i.e., multiple points of entry into the system). The execution path concept also permits interaction with neutronics codes other than ANISN such as DOT ${ }^{5}$ and VENTURE. ${ }^{6}$ Additional information on this interaction is available in the FORSS user's guide.'

Sensitivity analysis requires access to partial cross sections. The proposed CCCC file MATXS ${ }^{B}$ is the cross-section data base for JULIET. The preparation of a MATXS file is discussed in the FORSS user's guide.

The end product of JULIET is sensitivity coefficients (profiles). They are placed in a proposed $C C C C^{9}$ file, SENPRO. 10 A SENPRO Service Module ${ }^{7}$ is available in the FORSS system which operates on a SENPRO file providing a number of functions such as listing, plotting, merging, etc. SENPRO files are input for sensitivity analysis modules such as SENTINEL, II CAVALIER, ${ }^{12}$ and UNCOVER. ${ }^{13}$ 


\section{EXECUTION PATHS}

JULIET has four execution paths and the combination selected is problem-dependent.

\section{A. Operator Definition}

The path is selected by the word, MIX. This path prepares the crosssection operators required for the probleis solution (these correspond to cross sections appearing in the response definition and/or cross sections in the terms of the Boltzmann equation). The MATXS cross-section file is the primary data base for operator definition. User input defines the operatjrs required for the problem solusion. An operator may be a single material-reaction type or it may be a mixture of many materials and include several reaction types.

\section{B. Flux Preparation}

The path is selected by the word FLUX. This path calculates moment fluxes from existing forward and adjoint angular fluxes for use in computing certain reactor parameters (e.g., worth). This path also prepares the $\left\langle\phi^{\star} \phi\right\rangle,\left\langle\Gamma^{\star} \phi\right\rangle$, or $\left\langle\Gamma^{\star}\right\rangle$ inner products needed for a sensitivity calculation.

\section{Source Response Calculation}

The path is selected $D$ the word SOURCE. The path has the capability to calculate bilinear sources, linear sources, respunses, and normalization parameters. 


\section{Sensitivity Coefficient Generation}

The path is selected by the word SENSE. This path calculates sensitivity coefficients using the SWANLAKE algorithm expanded to include direct effects.

\section{OPERATOR DEFIN ITION}

The FORSS system is an attempt to provide a sensitivity analys is capability with respect to a response defined as:

for bilinear $R=\frac{\left\langle\phi^{\star} H_{1} \phi\right\rangle}{\left\langle\phi^{\star} H_{2} \phi\right\rangle}$, for linear $R=\frac{\left\langle H_{1} \phi\right\rangle}{\left\langle H_{2} \phi\right\rangle}$ with sensitivity coefficients defined as: $\frac{\partial R / R}{\partial \Sigma_{x} / \Sigma_{x}}$ where $\Sigma_{x}$ is a cross section appearing in the Boltzmann equation or in the definition of $H_{1}$ or $H_{2}$. In the above equations $\phi$ and $\phi^{\star}$ represent the forward and adjoint fluxes, the brackets denote integration over phase space, and the operators $H_{1}$ and $H_{2}$ can in general de arbitràry. However, the physical cases of most practical interest occur when either $H_{1}$ or $H_{2}$ is a macroscopic cross section, in which case $R$ would represent a weighted reaction rate ratio. For example, the iinear response ${ }^{28} \delta$ would be defined as $R=\frac{\left\langle{ }^{238} \Sigma_{c} \phi\right\rangle}{\left\langle{ }^{235} \Sigma_{f} \phi\right\rangle}$; clearly $H_{I}$ is the ${ }^{238} \mathrm{U}$ capture reaction and $\mathrm{H}_{2}$ is the ${ }^{235} \mathrm{U}$ fission reaction. Operators may also be linear combinations of any or all terms appearing in the Boltzmann equation. For example, the bilinear response for Na worth would be defined as:

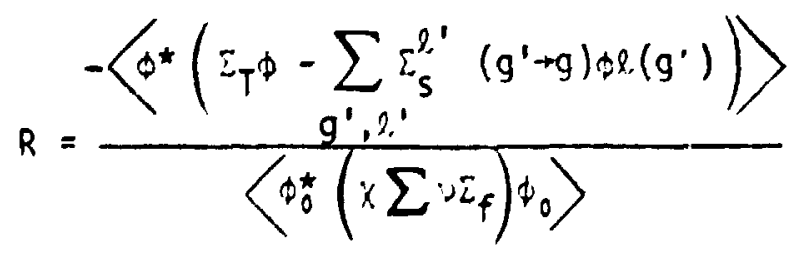




\section{VII-7}

Here $H_{:}$is the Boltzmann operator for $\mathrm{Na}$, and $H_{z}$ is the fission onerator for all fissionable materials in the system of interest.

IV. FLUX PREPARATION

\section{A. Moment Fluxes}

Angular fluxes are input to a JULIET calculation. Moment fluxes are prepared for use in source, response, and sensitivity caiculations according to the following equation:

$$
\phi\left(j, g, \ell^{\prime}\right)=\sum_{i=1}^{N O A} w_{i} \gamma_{\ell}^{m}\left(\mu_{i}\right) \hat{\psi}\left(j, g, L_{i}\right)
$$

where

ISCT - order of scattering,

NMOM - maximum of (ISCT, ISN),

NOA - number of angles,

for slab and spherical geometry $N O A=I S N+$ ?

for cylindrical geometry NOA $=(I S N *(I S H+4)) / 4$

ISN - quadrature order,

IMOM - number of moments,

$\ell^{\prime} \quad$ - moment index which assumes valles from 1 to LMOM. :"

is a function of $m$ and 2. Equations 4,6 , and 7 illustrate the relationship of $\ell^{\prime}, \ell, m$ as implemented in the code.

g - group index,

i - angle index,

$w_{i}$ - quadrature weight (infut, see Section VI, Eluck !),

$H_{i} \quad$ - discrete angle cosine (input, see Section :I, fiock 1),

$Y_{\ell}^{m} \quad$ - spherical harmonic, 
$\phi\left(j, g, \mu_{j}\right)$ - angular flux calculated by FANISM,

$\phi\left(j, g, \ell^{\prime}\right)$ - angular flux moment calculater by FANISN.

\section{B. $\left\langle\phi^{\star} \phi\right\rangle$ Files}

$\left\langle\phi^{\star} \phi\right\rangle$ files are prepared for use in the sensitivity equations. $\left\langle\phi^{\star} \phi\right\rangle$ is a syrbol ic term since the file may represent products of $\left\langle\Gamma^{\star} \phi\right\rangle$ or $\left\langle\Gamma \phi^{\star}\right\rangle$. $A\left\langle\phi^{\star} \phi\right\rangle$ file is required for $K$ and worth sensitivity analysis. औ $\left\langle\left[\Gamma_{\phi}\right\rangle\right.$ file is required for reaction rate ratio sensitivity analysis. $\left\langle\phi^{*} \phi\right\rangle$ files are prepared in the ANISN cross-section format. For the oth moment the total position of the $\left\langle\phi^{\star} \phi\right\rangle$ file is calculated as:

$$
\operatorname{PS}(I H T, g, Z, 1)=-\sum_{j \in Z} V(j) \sum_{i} \phi\left(j, g ; \mu_{j}\right) \phi^{\star}\left(j, g, i_{j}\right) W(i)
$$

where

IHT - position of total cross section in ANISN cross-sect:on table,

g - group index,

2 - zone index,

$R$ - the radi $i$ by interval boundary,

$V(j)$ - volume of spatial interval $j$,

$=R(j+1)-R(j)$ for slab,

$=\left(R^{2}(J+1)-R^{2}(j)\right)$ for cyl inder,

$=\frac{4}{3}\left(R^{3}(j+1)-R^{3}(j)\right)$ for sphere.

For slab and spherical geometry and the $P_{0}$ and $P_{1}$ scattering orders of cylindrical geometry the scattering positions are

$$
\operatorname{PS}\left(G, g, z, g_{-}\right)=\sum_{j \in \mathcal{E} Z} \phi\left(j, g^{\prime}, \ell^{\prime}\right) \phi^{*}\left(j, g, \ell^{\prime}\right) V(j)
$$


where

$\mathbf{G}=\mathrm{IHT}+\mathbf{g}^{\prime}+1$,

IHT is the total cross-section position,

$\ell=\ell$ assumes values of $l$ and 2 .

For cylindrical geometry and scattering order higher than $P_{2}$

$$
\operatorname{PS}(G, g, z, l)=\sum_{\ell^{\prime}=L L(\ell-1)}^{L L} \sum_{j \in Z} \phi\left(j, g^{\prime}, \ell^{\prime}\right) \Phi^{\star}\left(j, g, \ell^{\prime}\right) V(j)
$$

initially the code defines $L L(2)=2$ then $L L(\ell)=L L(\ell-1)+1+\frac{(2 \ell-1)}{4}$

where

$\ell$ is the scattering order index and assumes values from 3 to ISCT

(order of scattering) $\ell^{\prime}$ is the moment index.

v. SOURCE RESPONSE ALGORITHMS

A. The $\left[\mathrm{H}_{1} \phi\right]$ and $\left[\mathrm{H}_{2} \phi\right]$

Components of a $8 i l i n e a r$ Response

The $\left[H_{:} \Phi\right]$ function includes three terms, a total term, a scattering term, and a fission term.

1. Total

$$
T_{1}\left(j, g, \ell^{\prime}\right)=-\Sigma_{T}(j, g) \phi\left(j, g, \ell^{\prime}\right)
$$

where

$$
\begin{aligned}
& j \text { - spatial index, } \\
& g \text { - group irdex, } \\
& \ell^{\prime} \text { - moment index, } \\
& \Sigma_{T} \text { - total cross section, } \\
& \phi \text { - forward moment flux. }
\end{aligned}
$$


2. Scattering for Slab and Spherical Geometrics

then $\ell^{\prime} \leq$ ISCT, ISCT $=$ order of scattering

$$
T_{2}\left(j, g, x^{\prime}\right)=\sum_{g^{\prime}} \Sigma_{s}\left(j, g^{\prime}+g, \ell^{\prime}\right) \&\left(j, g^{\prime}, g^{\prime}\right)
$$

where

$\Sigma_{s}$ - scattering matrix.

\section{Scattering for Cylindrical Geometry}

For the $P_{0}$ and $P_{1}$ scattering orders Eq. (5) also applies for cyl indrical geometry.

For scattering orders greater than $P_{1}$ Eq. (6) and Eq. (7) establish the correspondence between the cross-section scattering order (index $\ell$ ) and the flux moment (index $\ell$ ').

$$
T\left(j, g, \ell^{\prime}\right)=\sum_{g^{\prime}} \Sigma_{s}\left(j, g^{\prime}+g, \ell\right)_{\phi}\left(j, g^{\prime}, \ell^{\prime}\right)
$$

To define the values of $\ell$ and $\xi^{\prime}$ in Eq. (6), initially the code defines $\ell=3$ and the indexing control parameter $\mathrm{LHI}=4$. Then as $\ell \cdot$ assumes values from 3 to LMOM (number of moments) the values of $\ell^{\prime}$ and LHI are compared. If $\ell^{\prime} \leq L H I$, the values of $L H I$ and $\ell$ are unchanged. When $\ell^{\prime}>L H I, \ell=\ell+1$, and LHI is redefined by Eq. (7) as follows:

$$
L H I=L H I+1+(2 l+1) / 4
$$

when $\ell>I S C T$

$$
T_{2}\left(j, g, \ell^{\prime}\right)=0
$$




\section{Fission}

$$
\text { For } \ell^{\prime}=1
$$

$$
T_{3}\left(j, g, \ell^{\prime}\right)=\lambda x(g) \sum_{g^{\prime}} v \tau_{f}\left(j, g^{\prime}\right) \phi\left(j, g^{\prime}, \ell^{\prime}\right)
$$

where

$$
\begin{aligned}
& \lambda=\frac{1}{k} \\
& x=\text { fission spectrum. }
\end{aligned}
$$

For $\ell^{\prime}>1$

$$
T_{3}\left(j, g, \ell^{\prime}\right)=0
$$

5. The $\left[H_{1} \phi\right]$ Term

$$
\left[H_{1} \phi\right]\left(j, g, \ell^{\prime}\right)=T_{1}\left(j, g, \ell^{\prime}\right)+T_{2}\left(j, g, \ell^{\prime}\right)+T_{3}\left(j, g, \ell^{\prime}\right) \text {. }
$$

6. $\left[\mathrm{H}_{2} Q\right]$ is a fission function with one term.

For $\ell^{\prime}=1$

$$
\left[H_{2^{\dagger}}\right]\left(j, g, l^{\prime}\right)=x(g) \sum_{g^{\prime}} \cup \Sigma_{f}\left(j, g^{\prime}, \hat{x}^{\prime}\right) ;\left(j, g^{\prime}, \ell^{\prime}\right) .
$$

For $\left[\mathrm{H}_{2} \phi\right], v \Sigma_{f}$ is a mixture of all fissionable materials in the assembly. For $\ell^{\prime}>1$

$$
\left[H_{2} \phi\right]\left(j, g, \ell^{\prime}\right)=0 .
$$

B. The $\left[H_{1}^{\star} \psi^{*}\right]$ and $\left[H_{2}^{*}+*\right]$

Components of a Bil inear Response

The $\left[H_{1}^{*} \phi^{\star}\right]\left(j, g, \ell^{\prime}\right)$ function has the same form as the $\left[H_{1}, j\right.$ function.

1. Total

$$
\left.T_{1}\left(j, g, \ell^{\prime}\right)=-i_{T}(j, g)\right\rangle^{\star}\left(j, g, \ell^{\prime}\right)
$$




\section{V!I-12}

\section{Scattering}

$$
T_{2}\left(j, g, \ell^{\prime}\right)=\sum_{g^{\prime}} \equiv_{s}\left(j, q \rightarrow g^{\prime}, i\right) g^{\star}\left(j, g^{\prime}, g^{\prime}\right)
$$

The discussion of geometry and monents for the $\left[\mathrm{H}_{2}+\right]$ function applies for the $\left[H_{1}^{\star} \phi^{\star}\right]$ function.

3. Fission

$$
T_{3}\left(j, g, \ell^{\prime}\right)=v \Sigma_{f}(j, g) \sum_{g^{\prime}}\left(g^{\prime}\right) \epsilon^{\star}\left(j, g^{\prime}, \Omega^{\prime}\right)
$$

4. The $\left[H_{i}^{*} \phi^{*}\right]$ Term

$$
\left[H_{1}^{*} \phi^{*}\right]\left(j, g, \ell^{\prime}\right)=T_{1}\left(j, g, \ell^{\prime}\right)+T_{2}\left(j, g, \hat{i}^{\prime}\right)+T_{3}\left(j, q, x^{\prime}\right)
$$

5. The $\left[\mathrm{H}_{2}^{*} 0^{*}\right]$ function is very similar to the $\left[\mathrm{H}_{2}+\frac{1}{2}\right]$ function.

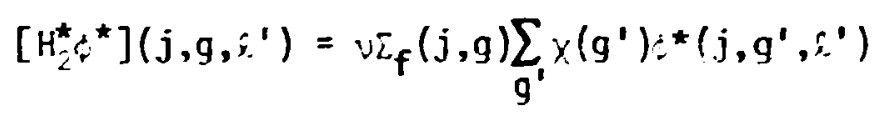

C. The Bilinear Response

A bilinear response is defined as:

$$
R \equiv \frac{\left\langle e^{\star} H_{1} \hat{\psi}\right\rangle}{\left.\left\langle\hat{t}^{\star} H_{2}\right\rangle\right\rangle}
$$

where

$$
\begin{aligned}
& \left\langle\dot{*}^{*} H_{1} \phi\right\rangle=\sum_{\ell^{\prime}} \sum_{g} \sum_{j} \phi^{\star}\left(j, g, \ell^{\prime}\right)\left[H_{j} \phi\right]\left(j, q, \ell^{\prime}\right) V(j), \\
& \left\langle\phi^{\star} H_{2} \phi\right\rangle=\sum_{g} \sum_{j} \phi^{\star}(j, g, l)\left[H_{2} \phi\right](j, \eta, l) V(j), \\
& \left\langle\phi H_{3} \star^{*}\right\rangle=\sum_{\chi^{\prime}} \sum_{g} \sum_{j} \phi\left(j, g, \ell^{\prime}\right)\left[H_{1}^{*^{*}} *^{*}\right]\left(j, g, \lambda^{\prime}\right) V(j), \\
& \left\langle\phi H_{2} \star^{*}\right\rangle=\sum_{q} \sum_{j} p(j, q, 1)\left[H_{?}^{*},-\right](j, q, I) V(j) .
\end{aligned}
$$




\section{13}

D. The Bilinear For saro Source

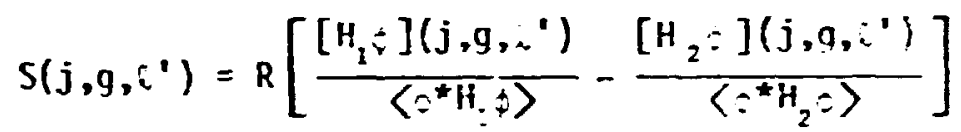

E. The Bilinear Adjoint Source

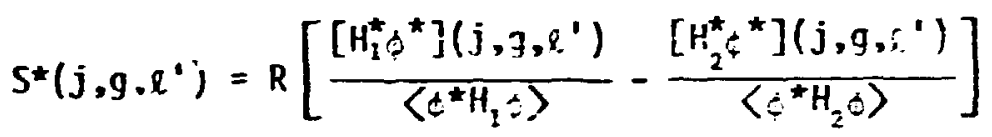

F. The Hurth in an Interval

Reactivity worth, $w$, is defined as:

$$
w(\text { per mole })=R\left[\frac{.6923}{N V}\right]
$$

$R$ - the bilinear response.

$V$ - volume of the interval.

$\mathbf{N}$ - density of the material.

$$
w(\text { per kilojram })=\frac{1700}{A} \quad[w(\text { per mole })]
$$

A - the atomic mass.

\section{G. The Linear Response}

A 1 inear response, $R$, is defined as:

$$
P \equiv \frac{\left\langle H_{1} \cdot \dot{*}\right\rangle}{\left\langle H_{2} \cdot \dot{ }\right\rangle}
$$

where

$$
\left\langle H_{i} ;\right\rangle=\sum_{y} \sum_{j} H_{i}(j, \tau) e(j, q, 1) V(j)
$$


VII -14

$$
\left\langle H_{2}, s\right\rangle=\sum_{g} \sum_{j} H_{i}(i, g):(j, g, l) V(j)
$$

$30 i$

where

$H_{1}$ and $H_{2}$ are simply reaction cross sections or other response functions,

$=(j, q, 1)$ - Ooh moment flux,

$g$ - group index,

j - spatial index.

H. The Linear Source

$$
S^{*}(j, g)=R\left[\frac{H_{1}(j, g)}{\left\langle H_{2}, \partial\right\rangle}-\frac{H_{2}(j, g)}{\left\langle H_{2} \hat{g}\right\rangle}\right]
$$

VI. GENERATION OF SENSITIVITY COEFFICIENTS

JULLET implements the sensitivity equations discussed in Section III of Or:BL/TM-5563.: The equation below is solved to yield the sensitivity of response to a cross section as defined by an operator. A common form of the operator is a single material--reaction type. However, the operator can take the form of a cross-section mixture. In the equation. the cross-section operator of interest is designated by the subscript $x$.

A. General Representation of Terms in Sensitivity Coefficient Definitions

$$
\begin{aligned}
& T_{1}(g, z)=-z_{x}(g, z) \sum_{j \in Z} V(j) \sum_{k=1}^{N O A} *(j, g, k) \phi(j, g, k) w(k) \\
& T_{z}(g, z)=\frac{1}{k} \sum_{j \in z} V(j)\left[v(g, z) \tau_{f}(g, z) \partial(j, g, 1) \sum_{q^{\prime}=1}^{I G M} e^{* \prime}, j, g^{\prime}, 1\right) ;\left(g^{\prime}, z\right)
\end{aligned}
$$




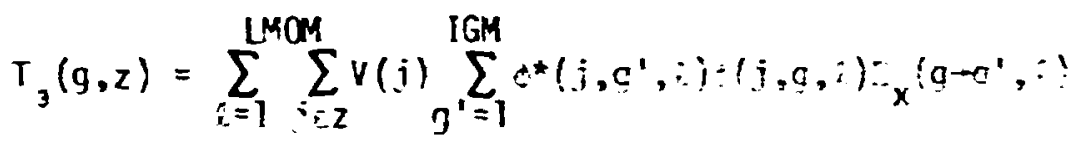

$$
\begin{aligned}
& \left.T_{4}(g, z)=\Sigma_{x}(g, d) \div(d, g, 1) /\left\langle H_{1},\right\rangle\right) \\
& T_{4}(g, z)=0 \text { unless } \Sigma_{x} \text { appears in the definition of } H \text { : } \\
& T_{5}(g, z)=-\Sigma_{x}(g, d):(d, g, 1) /\left\langle H_{z} \Leftrightarrow\right\rangle
\end{aligned}
$$

$$
T_{5}(g, 2)=0 \text { unless } \Sigma_{x} \text { appears ir the definition of } H_{2}
$$

The $d$ indicates the space increment where the direct effect $\neq 0$ i.e., where $H_{1}$ and $H_{2}$ are defined).

$$
\begin{gathered}
T_{i}(g, z)=T_{s}(a, z)=0 \text { jf } d \text { is not within zone } z \\
P(g, z)=\frac{1}{k}\left[T_{i}(9,2)+T_{2}(g, z)+T_{3}(g, z)\right]+T_{-}(0, z)+T_{5}(0, z)
\end{gathered}
$$

where

$$
\begin{aligned}
& \text { g - group index, } \\
& g^{\prime} \text { - group index, } \\
& z \text { - ane index, } \\
& \text { j - spatial interval index, } \\
& k \text { - angle index, } \\
& v(j) \text { - volume of interval, } \\
& { }^{2} x(9, z) \text { - cross section operator, } \\
& \phi(j, g, k) \text { - adjoint anquiar flux. } \\
& :(j, g, k) \text { - forward angular flux. } \\
& w\{k\} \text { - discrete ordinates weingt. }
\end{aligned}
$$




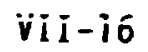

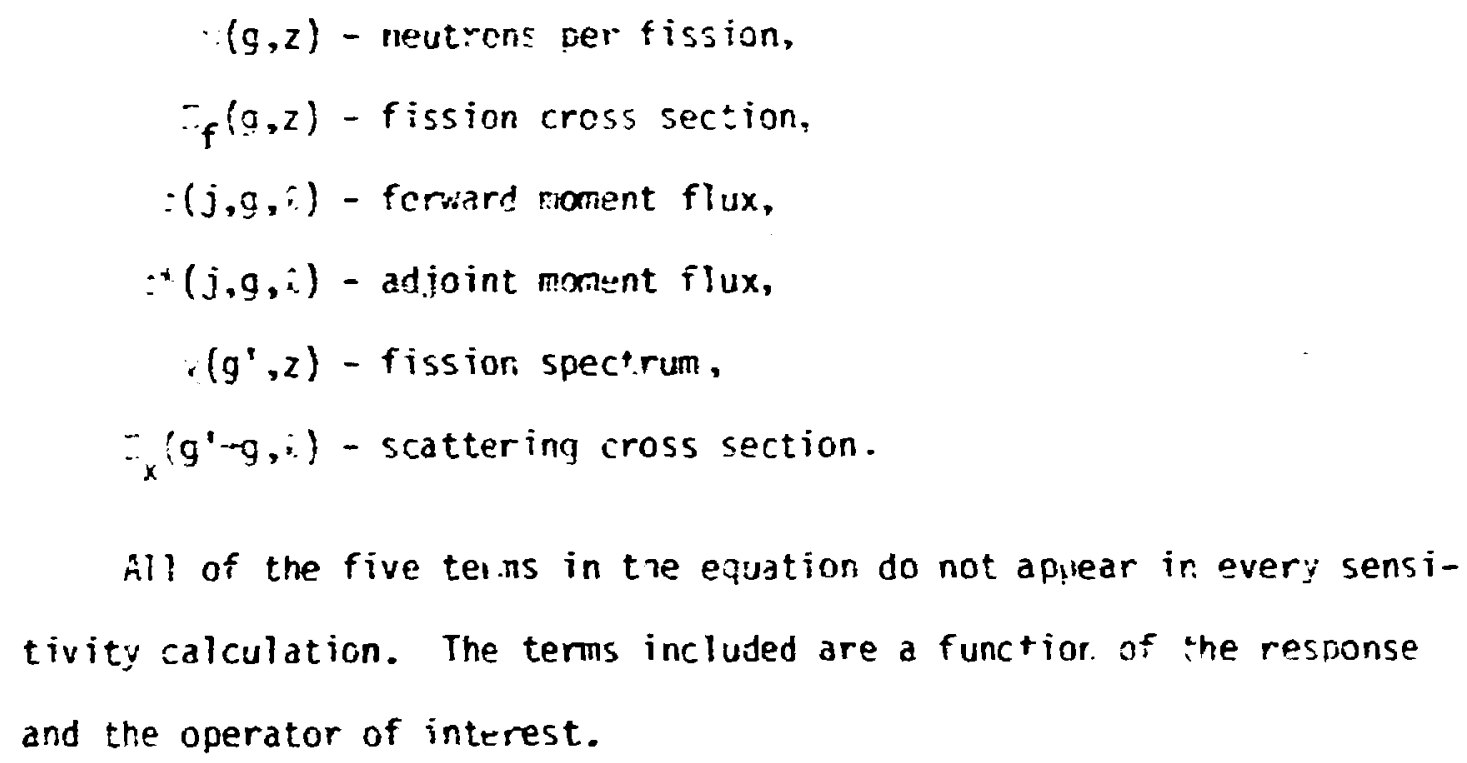

fill of the five teins in the equation do not apulear in every sensitivity calculation. The tenms included are a functior of the response and the operator of interest.

\section{B. K Sensitivity}

1. Assorption Reactions, Nonfi jsiotiab:e, ivonscattering Reactions such as Canture

The equation reduces to $T_{1}(g, z)$ where $\sum_{x}(g, z)$ is the ausorotion cros: sectic- of the naterial of interest.

\section{Fission Peactions}

The fission rea tion requires only $T_{:}(q, z)$ and $T_{:}(g, z)$. In $T_{:}$, $z_{x}(f, z)$ is the fiss:in cross section of the material of interest.

\section{Sensitivity}

The: reaction requires only $T_{2}(7, z)$.

\section{Scattering Reactions}

if scattering reaction such as elastic requires $T_{1}(q, ?)$ and $T_{1}(,, z)$ wnere $\tau_{x}(g, z)$ in $T_{1}$ is the elastic scattering cross section. $\bar{x}_{x}\left(q^{\prime} \rightarrow q, \therefore\right.$ ! in $T_{3}$ is the elastic group-to-group scattering matrix for moment: of the material of interest. 


\section{Total Reaction of a Fissiunable liaterial}

The total reaction of a fissicrable material requires the $::(c, z)$, $T_{2}(g, z)$ and $T_{3}(g, z)$ terms. $\bar{z}_{x}(g, z)$ in $T_{1}$ is the total cross section and $\Sigma_{x}\left(g^{\prime}+g, 8\right)$ is the total group-to-group scattering matrix.

\section{Total Reaction of a Nonfissionabie Haterial}

The total reaction of non-fissionable materials is the sane as (5) except the $T_{2}(g, z)$ term is 0 .

The $T_{4}$ and $T_{5}$ terms do not enter into a $k$ sensitivity since they are direct-effect expressions for reaction rate ratic responses. For $k$ sensitivity, the $R$ in Eq. (37) is to be interpreted as $P=:\left\langle i * !_{2}:\right\rangle$.

\section{Reaction Rate Ratios}

When the response is a reaction rate ratio such as : : $=0$ casture $:$ : fission, the ${ }^{2 E} \mathrm{c} /{ }^{25 f}$, both indirect and direct effects must be consigered. Direct effects occur only when the operator of interest is a material reaction type which is a constituent of the response defirition. iil operators have indirect effects.

\section{Indirect Effect}

The algorithm is identical to $k$ sensitivity with : reilacing :* in Eq5. (32). (33) and (34), and the $R$ appearing in Eq. (37) is to be interpreted as:

$$
R=\frac{1}{5}\left[\frac{\langle!: \vdots\rangle}{1+:\rangle]}\right]
$$

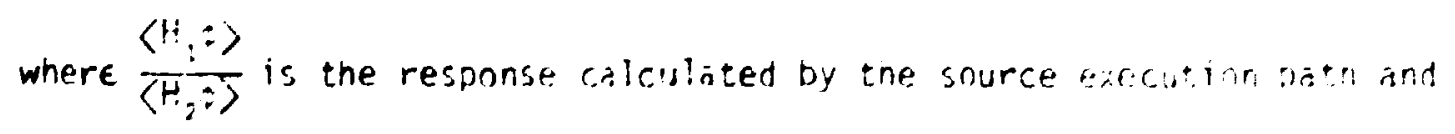

$S$ is the source nomalization from the AldSA generalized thioine calculation. 


\section{H. Oirect Esfect}

when the onerator of interest is the material-reaction iype which forms the numerator of the reaction rate ratio, the $H_{1}$ direct effect is calculated and sumiced with the indirect effect to conplete the profile. The $H_{i}$ direct effect is calculated by the $T_{-}(9,2)$ term in Eq. (37). For the example, $Z_{x}(g, z)$ in $T_{2}$ is the $23 e_{U}$ capture cross section. $\left\langle H_{1} A\right\rangle$ is available from the source execution path. The $T_{-}$term and $\left\langle H_{i} \Leftrightarrow\right\rangle$ must be calculated nver the same space; frequently this is the central interval. $i$ is zero in any zone that does not include the space increment used in the $\left\langle\mathrm{OH}_{1}\right\rangle$ calculation.

\section{3. $H_{2}$ Direct Effect}

When the overator of interest is the material-reaction type which forms the denominator of the reaction rate ratio, an $\mathrm{H}_{2}$ direct effect is calculated and surmed with the indirect effert to complete the profile. The $H_{2}$ dirert effect is calculated by the $T_{\subseteq}(g, z)$ term in Eq. (37): For the example, $\bar{z}_{x}(g, z)$ in $T_{\leq}$is the $: 3 y$ fission cross section. $\left(H_{2}, ?\right.$ is avsilable from the source execution path. The $T$, term and $\left\langle H_{2} \Rightarrow\right.$ m.jst be calculated over sta same space; frequently this is the central interval. T, is zero in any zone that does not include the space increment used in the $\left\langle\mathrm{H}_{2} \Rightarrow\right.$ calculation.

\section{Worth Sensitivities}

The following is based on a definition of the worth $R$ of a material : in region: of the form

$$
\begin{aligned}
R & =\frac{N}{k^{2}} \frac{\partial k}{\partial N} \\
& =-N \frac{\partial \lambda}{\partial N}
\end{aligned}
$$


where the eigenvalue $:$ is equal to $l, k$ and $N$ is the density of material in region $\rho$. On the basis of generalized perturbation theory, an equivalent expression for $R$ is

$$
R=\frac{\left(\omega * H_{1} \omega\right)}{\left(\hbar \star H_{2} \phi\right)}
$$

where operators $H_{1}$ and $H_{2}$ are given by

$$
H_{1}=-A_{N}+: B N
$$

and

$$
H_{2}=B
$$

The Boltzmanr operator $L i$ : of the form $A \cdots \lambda$ and 0perator $B$ is that part of the Boltzmann operator which depends on $\ddot{r}_{f} \cdot B_{l l}$ is that ce tribution to $B$ from material $;$ in region $\therefore A_{N}$ is the contribution to the operator $A$ from material $L$ in regiun o and consists of total and scattering crcss section terms. The "unperturbed" flux and adjoint flux are given by : and : respectively. Sensitivities of a worth response may be calculated with respect to any material-reaction type. Let this type be indicated by : . If the material of type: is the same as the material:. which defines the worth response, then these sensitivities have direct-effect contributions and indirect-effect contributions. In general, there are five contributions to a sensitivity: Two are direct-effect contributions, and three are indirect effects. The five contributions are:

1. $H_{1}$ direct effect

2. $\mathrm{H}_{2}$ direct effect

3. Eigenvalue indirect effect

4. Forward flux ind: rect effect

5. Adjoint flux indirect effect

If material of type $f$ is different from material $\ldots$, then only indirect 
effects are nonzero and contribute to the ensitivities. The $\mathrm{H}_{2}$ direct effect is nonzero only for fissionaole material-reaction types.

1. $H_{1}$ Direct Eifect

Th. $s$ effect is defined as $\frac{\left\langle\phi^{\star} H_{1} \phi^{\dagger}\right\rangle}{\left\langle\phi^{\star} H_{1} \phi\right\rangle}$ where $H_{1}$ is that part of the $H_{1}$ operator which consists of the cross sections of the matterial-reaction type $a$ in the region of interest. The sensitivity of every reaction type $c$ f the material $\mu$ appearing in the response definition will have an $H_{1}$ direct effect contribution.

2. $\mathrm{H}_{2}$ Direct Effect

This effect is defined as $-\frac{\left\langle\phi^{\star} H_{2 a} \phi\right\rangle}{\left\langle\phi^{\star} H_{2} \phi\right\rangle}$ where $H_{2 a}$ is that part of the $H_{2}$ operator which consist, of the fission operator for the materialreaction type $a$ with the spatial dependence of the material in the assembly. The sensitivities of the chi, nubar, and fission reactions of the material $\mu$ appearing in the iespun dese definition have an $\mathrm{H}_{2}$ direct effect contribution.

\section{Eigenvalue Indirect iffect}

This effect is defined as

$$
\left[-\frac{1}{R} \frac{\left\langle\phi^{\star} A_{N} \phi\right\rangle}{\left\langle\phi^{\star} H_{2} \phi\right\rangle}-1\right] S_{a}^{k}(g)
$$

$s_{7}^{k}(g)$ is the group dependend $k$ sel.sitivity of the material-reaction type of interest.

The sensitivity of every reaction tye will have an eigenvalue direct effect contribution.

4. Forward Fiux Effect

This effect is defined as $\frac{\left\langle\Gamma^{*} L a \phi\right\rangle}{\frac{R}{S}^{\star}}$ where $L a$ is that part of the Boltzmann operator due to cross sections for the material-reaction type \& to 
which sensitivities are being calculated. $L_{a}$ is defined over all spatial regions in which the material of type a occurs in the system. $S^{*}$ is the source normalization calculated jy FORSS ANISN for the case which generated $I^{*}$. Computationally JULIET solves the same equation used for $k$ sensitivity with the adjoint flux $\phi^{*}$ replaced by the generalized adjuint fi':x $\Gamma^{\star}$ and $\lambda<\xi^{\star} H_{2} \phi^{\prime}$ replaced by $R / S^{\star}$.

5. Adjoint Flux Effect

This effect is defined as $\frac{S \Phi^{\star} L a I>}{\frac{R}{S}}$ where $L a$ is, as above, that part of tne Boltzmann operator cue to cioss sections for the material-reaction. type $a$ to which sensitivities are being calculated. $L a$ is defined over all spatial regions in which the material occurs in the system. $S$ is the source normalization calculated by FORSS ANISN for the case which generated $\Gamma$. Computationally JULIET solves the same equation used for $k$ sensitivity with the forward flux $\phi$ replaced by the generalized forward $f l u x I$ and $\lambda\left\langle\phi^{\star} H_{2} \phi\right\rangle$ replaced by $R / S$.

\section{JULIET INPUT PREPARATION}

JUL LT performs two major functions, source generation anc the calculation of sensitivity coefficients. A single computer run may be set up to accomplish both functions or they may be done independently. Formally a source calcuiation must precede a sensitivity calculation since source generates normalization parameters used in the sensitivity calculation.

\section{A. Execution Paths}

JULIET is divided into four execution paths. Path selection is accomplished by the user to solve the problem of interest. The paths are: 


\section{Operator Uefinition, Selected by the Vord, MIX}

This path must always be executed because operators are required for any JULIET function. Operators are defined from crnss sections. They may have the form of a single material reaction cross-section type, a mixture of a single reaction from several materials or other combinations of materials and reaction cross-section types. A common operator is a mixture or material which includes $\Sigma_{c}, x, \Sigma_{f}, \Sigma_{a}, \Sigma_{f}, \Sigma_{T}$ and the total: :oup-to-group scattering matrix. This is referred to as an automatic operator in JULIET. It is used to form both the $H_{2}$ and $H_{2}$ bilinear operators in a SOURCE calculation. When an automatic operator is referenced in a sensitivity càlrulation, sensitivity coefficients are calculated for the capture, fission, $\bar{v}, x$, and total reactions.

2. Flux Preparation, Selected by the hord, FLUX

The path calcilates moment fluxes from angular fluxes and also prepares the so-called $\phi^{\star} \phi$ file used by the sensitivity calculation. Actually, the file may also be $\Gamma^{\star} \phi$ or $\Gamma \phi^{\star}$. The execution of this path is prerequisite to a SOURCE-RESPONSE calculation. The executio.l of the path may be omitted when going directly to the calculation of sensitivity coefficients and appropriate $\phi^{*} \phi$ i iles already exist.

\section{Source Generation, Selected by the Word, SOURCE}

This path is executed to calculate sources, responses and normalization parameters. The equations solved are outlined in Section IV. Do not execute this path in stud'es using two-dimensioral sensitivity analysis. 


\section{VII -23}

\section{Sensitivitv Coefficient Generation, Stlected by the Hord, SEN SE}

This path implements the sensitivity coefficient generation algorithms outlined in Section $V$.

\section{B. Input Blocks}

Since JULIET input is a function or the execution paths selected, the input, is divided into five distinct biocks. All of the blocks ds not have to appear in a run.

\section{Block 1 (always provided)}

The content of Block 1 is a function of the execution paths seiectes. If FLUX is a member of the execution path, Block 1 input is:

1555 Integer Paraneters [36]

1. Any integer

2. ITH -100 only downscatter cross rections; :100 upscatter

3. ISCT - maximum order of scatter found in any zone

4. ISN - order of angular quadrature

5. IGE - 1 - siab; 2 - cyl inder; 3 - sphere

6. Any integer

7. Any integer

8. IZH - number of zones

9. IM - number of mesh intervals

10. Any integer

11. IGM - number of energy groups

12. IHT - position of Jtotal in $\phi^{\star} \phi$ table

13. IHS - position of $\sigma_{g g}$ (self-scatter) in $\phi^{\star} \phi$ table

14. IHM - length of $q^{\star} \phi$ table 
15.

- Ary integer

$\cdot$

36.

$1 *$ Fission spectrum [IGH]

4 4* Radii by interval boundary $[I M+1]$

5*k Velocities [IGM], the array may be onitted

6** Arıgular quadrature weights [MM]

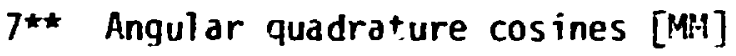

8SS Zone numbers by interval [IM]

$\mathrm{T}$

Note that Block 1 input is ANISN input with deletions and minor modifications. The $8 \$ \$$ caid describes the soatial dependence in the sensitivity calculation except for reaction rate ratio direct effects. The $35 \$$ card does account for the spatial dependence of worth direct effects and the card used in the AlISN calculation usuaily must be modified. All entries on the $15 \$ \$$ card are not used in JULIET, but the format is unchange: to permit the convenience of reproducing the card from existing tivisn input.

If FLUX is not a member of the execution rath, Block 1 input simplifies to:

1555 O ITH ISCT 42 IZM 22 IGM IHT IHS IHM FO $T$ 
VI I -25

where

ITH $<900_{i}^{\prime}>100$, no upscatter/upscatter,

ISCT maximum order of scatter,

IZM number of zones,

IGM number of energy groups,

IHT position of $\sigma_{\text {total }}$ in cross-sertion table.

IHS position of $c_{g q}$ (self-scatter) in cross-section table,

IHM length of cross-section table.

2. Block 2 (always provided) Operator Definition

This input defines the operators to be used in subseguent source and sensitivity calculations. A11 Block 2 input is in a fixed field format. Operators are defined as a function of cross sections and this input references the MATXS file.

MIX (columns 1-3) selects execution path.

Operator Definition Cards. Columns (1-5) (I5) Operator ID. This may be an arbitrary number assigned by the user. Ho. ever, if anplicable, an ENDF/B material identification number is suggested for this input. Columns (11-16) (A6) MATXS particle identifier, NEUT is normal input. Columns (21-26) (A6) MATXS type identifier, NSCAT is normal input. Columns (31-36) (26) MÁTXS material identifier. Columns (41-46) (A6) MATXS reaction type identifier/blank/ALLIML/ELSCAT or cisher reaction type name.

Columns $(51-60)(E 10.4)$ Density.

Columns $(61-65)(15)$ Order of $P_{n}\left(p_{0}-0\right)$.

Columns $(66-70)(15)$ if $\because n$, el iminates $\%$

When all operators are defined, insert 1 blank card. 


\section{VII -26}

Mixing. Operators are frequently defined as cross-section mixtures. A set of two or more operator definition cards with identical operator identifiers define a mixtuire which may be referenced in source and sensitivity calculations.

Comments on the Operator ID (Columns 1-5). Sensitivity coefficier.ts are placed in a proposed CCCC file SEIPRO. Part of the identification of a set of coefficients in SENPRO is the ENDF/B material identification. The operator ID is passed to SENPRO and used as the ENDF/B material identification number. If the operator is a mixture or somethirs eise that cannot be properly identified vitir an E:DF/: MAT number, the user must take approprizte action to insure proper identification and use of the data. For some applications, JULICT sabotages the SENFRO EHDF/B material identification scheme. Two or mors equal operztor ID numbers define a mixture. This can cause difficulty when several operators are defined for the same material but with different reaction types. Future code development will eliminate this problem. In the interim the ENDF!R material identification scheme must sometimes be abandoned and arivitrary unique operator IDs assigned. The SENPRO service module provides a cirick and easy method to redefine the number used as an ENDF/B material identification. This service may also aid the user with an operator that cannot be correct?y identified in ENDF/B terminology.

$$
\text { Comments on MATXS Reaction Type Identifier (Columns 41-46). Please }
$$

rote this is a six-character string, a word not an integer. The MATXS files currently in use were obtained by trans?ating either ANISN files or AMPX master files. In either case, the ENDF/B reaction type numbers (MT) influence the procedure. The translators establish the following relationship: 


\section{VII -27}

ENDF/B (HT)

1

2

3

4

16

17

18

19

20

21

51

:

90

91

102

452

1452
HATXS (Columns 41-46 entry)

TOTAL

ELSCAT

NONELS

INSTOT

N2N

N3N

FIS

FCF IS

SCFIS

TCF IS

INELOI

:

INEL40

CTNUIM

CAPT

NUBAR

NUF IS

For other ENDF/B MT numbers the translator forms a MATXS six-character string as follows: If the MT $=1234$, the MATXS identifier will be MT1234.

If columns (41-46) are left blank, an automatic operator is genericter.

If columns (41-46) are ALLINL, sensitivity ccefficients will be generated for all inelastic levels and the continuum.

Comments on Columns (66-70). If the operator is an automatic misture, this entry prevents a mixing operation on the fission spectrum. 
3. Black 3 (Optional)

FLUX Preparation

FLUX (Columns 1-4) selects execution path.

foss Integer Parameters [11]

KCI - 0/I, prepare $\delta \$ V$ for central interval/no effect. This parameter controls spatial dependence of the direct effect for reaction rate ratio calculations. Central interval direct effect is the nomal calculation. If a 1 is entered, then the direct effect spatial dependence is defined by the 855 card in Block 1 . If $K C I<0$, no $*_{*}^{*}$ file will be made.

IRF - identification number for forward flux.

IRA - identification number for adjoint flux.

LPATH - If <0 no adjoint flux will be read from repository.

KERT - Unit number of $\phi^{\star} \phi$ file, defadt $=9$.

F0 - reserved.

T

For both IRF and IRA, enter negative values wilen the adjoint flux component of a worth sensitivity is being calculated. This informs the code that the forward and adjoint fluxes are to be interchanged.' When IRF $=$ IRA $=0$, the adjoint flux used is identified by the FMANG identification number (second antry FMANG 155 card) and the forward flux is:.

4. Block 4 (Optional)

SOURCE Generation

SOURCE (Columns 1-6) selects exerution path.

70\$\$ Integer Parameter [1]

NRESP - number of resp unses. 


\section{VII-29}

$71 * *$ Floating point parameters [2]

$k$ - If $k=0$ is entered, the value is retrieved from the reposizor:. F0. 0

T

$\star \star \star$ Repeat the following title, 7255, 7355, 7455, 75**, T sequence of cards for each response

HOLLERITH TITLE of response (20

7255 Integer parameters [2+IM] where IM is the number of mesh intervals.

1. ILBL - operator type identifier.

0 - linear operator

1 - bilinear operator

2. ISCT - scattering order $\left(P_{0}=0\right)$.

3. $H_{1}$ operator identifier for each mesh interval. See B?ock 2 input for operator definition.

$:$

IM+2

7355 Integer Parameters [2+IM]

1. 0

2. 0

3. $\mathrm{H}_{2}$ operator identifier for each mesh interval.

:

:

$I M+2$

745s Integer Parameters [10]

1. LPRT - source print option.

$$
0 \text { - no print }
$$$$
1 \text { - print }
$$ 


\section{VII -30}

2. LSAVE - source save option ir. the reposicory.

$$
\begin{aligned}
& \text { 0 - no save } \\
& \text { I-save }
\end{aligned}
$$

3. IDA - adjoint source identification number.

4. IDF - forward source identification number.

5.

Fo, reserved for future options.

10.

$75^{\star \star}$ Omit if response is not a reactivity worth [2]

A - Atomic mass of material of interest.

B - Density of material of interest.

T

5. Block 5 (Optional)

Sensitivity Coefficient Ca! - ? etion

SEMSE (Columns 1-5) selects execution path.

$805 \$$ Integer Prameters [2]

NRESP - number of responses.

ILNG - last neutron group.

$T$

$81 * *$ Floxting point parameters [ILNG+i]

lieutron group structure.

82** Floating poirt parameters [NGG=1] where NGG is the number of $\because$ groups.

$\because$ group structure.

$T$ 


\section{$\mid I I-3 I$}

** Repeat the following sequence of cards enting : with o!s ? $5=0$ each response $* \star \star$

HOLLERITH TITLE FOR ASSEMBLY (20A4)

F.DLLERITH TITLE FOR RESFOWSE (20A4)

then the response is a reaction rate fror which direct effects will be calculated, extra care must be taker. with the response descriction. Colums (1-8) material ident; fier for the numerator, example $1 j-233$. Colums (9-16) reaction rat.e identifier for the numerator, example CAPT.

Column: (17-24) material identifier for the denominator.

Columns $(25-32)$ reaction rate identifier for the denominator.

NOTt: These material and reaction rate identifiers must be identic.l to their appearance in the operator definition cards, see data block 2 input. If + automatic operator was designated then the reaction type is blank on the operator definition cards and the identifiers used here must be identical to those assigned by the code, see page 24 .

\section{5s Integer Parameters [13]}

NE - number of operators tc be processed.

IRESF - response identifier for data retrieval fron resositor $\because$.

IDS - response identifier placed on SEAPRO.

NTRN - reaction rate ratio identifier Dlaced nr SE:IPP.O.

VERT - unit number of :*: file, default $=9$, default used trrer. is entered.

IPRO - unit number of SENPRO file. Default - 23, code will not allow a change under the response loop after the initial definition. 


\section{I -32}

Fo - reservec for iuture options.

$T$

86** Floating point parameters [6]

R - calculated value of response.

R1 - $\left\langle H_{1} \phi\right\rangle$, used only when direct effects are to be calculated; otherwise enter 0 .

R2 - $\left\langle\mathrm{H}_{2} \mathrm{O}\right\rangle$, used only when direct effects are to be calculated; otherwise enter 0 .

EK - $k$; if not applicable, enter 0.

EVR - measured value of response; if not applicable, enter ?.0.

FuT - enter 0; reserved for future option.

T

When $R, R 1, R 2$ and $E K$ are all entered as 0 , these parameters are retrieved from the repository.

****:-Repeat following sequence for NE operators

HOLLERITH TITLE for material-reaction (20A4)

87\$5 Integer Parameters [10]

1. LMAX1 - Order of $P_{n}$, enter 0 for $P_{0}$.

2. IFISS - Fission option.

0 - no fission

3 - total sensitivity of fissionable material

13 - sensitivity of fission cross section

23 - sensitivity of $\bar{v}$

3. IOP - Direct effect option.

0 - no direct effect

1 - material-reaction type in response numerator

2 - material-reaction type in response denominator 
4. NZP - number of entries on 9155 card.

5. JOFA(?! 9 - 0/I do not save/save indirect effect.

6. JOPA(2) 2 - 0/1 do not save/save direct effect.

7. JOPA(3) $)^{a}-0 / 1$ da not save/save indirect + direct effect.

8. INC $-0 / 1$ do nat include/include results in sumetion over all operators.

9. LCRW - 0 .

i?. LPRT - 0/1 normal output/limited output. Norma $i$ outplit

lists a sensitivity matrix which includes sensitivities by group and group-to-group transfers. Lirited output lists only total sensitivities by zone.

T

88\$S Integer Parameters [NZONE]

Operator identifiers by zone.

$89 * \star$ Floating point parameters [NZONE]

Zone densities.

9055 co $^{0}$ - reserved for future options.

$91 \$ \$$ Integer Parameters [NZP]

00 - loop limiters for zone sumations.

\footnotetext{
When rocessing responses that do not have direct effects, for example $k$, thon JOPA $(1)=1$, JOPA $(2)=J O P A(3)=0$ is sufficient to save the sensitivity coefficients on a SENPRO $f_{i}$ le. This is al so true when processing rEsponses that have direct effects but the material-reaction type being processed does not appear in the response definition, i.e. the direct effect sensitivity is 0 . In the above situations, avoid the conclusion that JOPA $(1)=$ JOPA(2) $0, J O P A(3)=1$ will sum the indirect effect and 0 and thus save the indirect effect sensitivities on a SENPRO file. It will not happen and the erergy dependent sensitivity coefficients will be lost.
} 
Example: $91 \$ \$ 112212$ will produce three sets of cuefficients: zone 1, zone 2, and a summation over zones 1 and 2 .

$T$

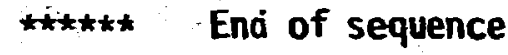

\section{RESOURCE UTILIZATION}

The amount of computer core required is highly problem-dependent. The parameters affecting core size requirements are listed in the discussion of the container array. The most important parameters are the number of groups and the lumber of spatial mesh intervals. The number if moments and ine scattering order have little impact on core requirements but are important factors in the amount of usage of exiernal storage devices such as disks and tapes. Computer CPU usage by JULIET is trivial relative to cross-section preparation and neutronics calcu? ations.

\section{A. Container Array}

Most data storage is in a single container array. The length of this array varies for each execution path. It is necessary to calculate the container array length for each execution path activated in a run. In the main program the length must be set to the maximum value calculated olus a common term IC. Parameters of inturest in determining contafner array length:

IGM - number of groups.

IM - number of space intervals.

NOA - number of angles. 


\section{VII -35}

NZ - number of zones.

ITL - ANISN cross-section table length.

IDP - precision parameter. IDP $=2$ for double-word precision. IDP $=1$ for single-word precision.

MKNT - number of materials in the bilinea $H_{1}$ operator.

M.LSA - length of the largest compressed scattering matrix in the problem.

ISCT - onder of scattering.

MS - total number of reactions available for sensitivity analysis.

NHI - number of materials in the linear $H_{1}$ operator.

NH2 - number of materials in the linear $\mathrm{H}_{2}$ operator.

\section{COMMON TERM}

$$
I C-132+C * I G M+3^{\star} I M+N Z
$$

\section{Operator Definition}

$$
\begin{aligned}
I C O= & M S^{\star}\left(2^{\star} I S C T+14\right)+2 \star 1 G M^{2}+I G M^{\star}(6+2 * I S C T)+M A X 0(7500, \\
& \left.I G M^{2}, 75^{\star} I G M\right)
\end{aligned}
$$

\section{Flux Preparation}

$$
\begin{aligned}
& I 1=2 \star(I M+1) \star N O A+I G M *(I M+N Z) \\
& I 2=I G M *(I T L+2 \star N Z+I T I) \\
& I 3=I G M *(I T L+2 \star N Z+2 * I M) \\
& I C F^{\prime}=\operatorname{MAXO}(I 1, I 2, I 3)
\end{aligned}
$$

\section{Source Response}

Bilinear

$$
14=I G M *(I M * I D P+M K N T+1)+M K N T+M Y S A+M S *(I S C T+2)
$$


VII -36

$$
\begin{aligned}
& I 5=I M * I G M *(2 * I D P+1) \\
& I C I=\operatorname{MAXO}(I 4, I 5) \\
& \text { Linear } \\
& I C 2=2^{\star} I G H *(N H I+N H 2)+M S+I M+I G M \\
& I C R=2^{\star} I M+4+M A X O(I C I, I C 2)
\end{aligned}
$$

\section{Sensitivities}

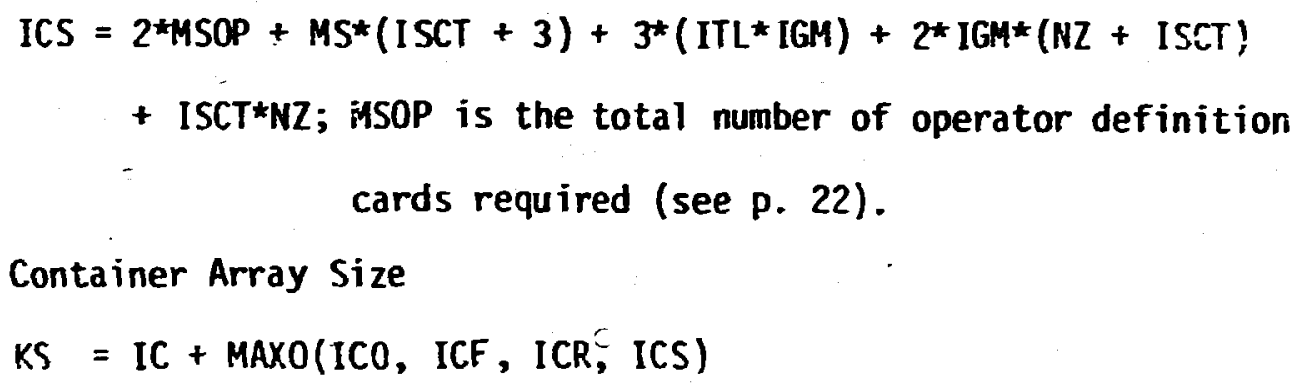

B. Overlay Structure

JULIET uses a simple overlay structure which could be made mere detailed if required. Basically each execution path is contained in an overlay segment. The sensitivity path user three overlay segments. The overlay structure is shown in Fig. 1. 


\section{VII-37}

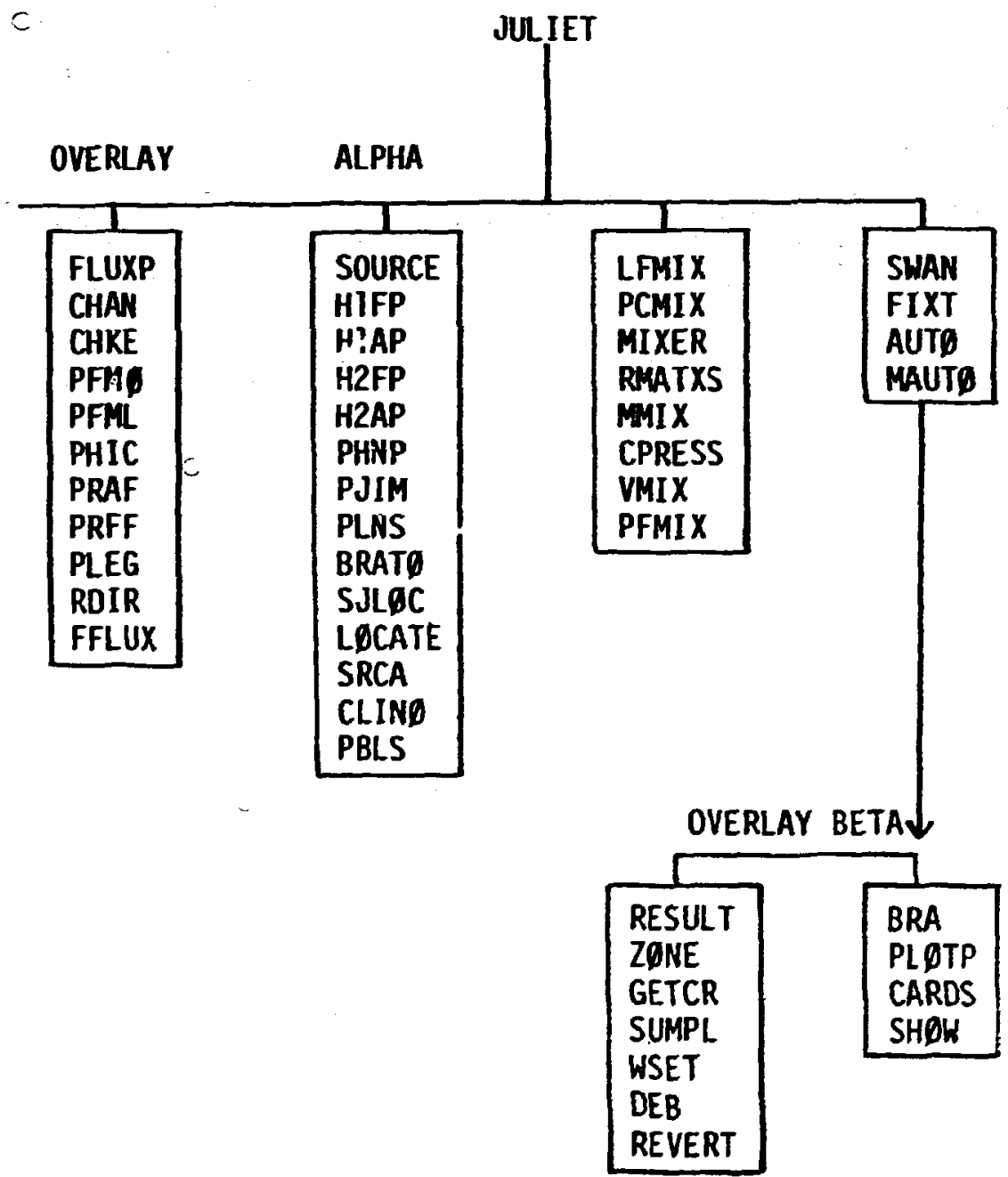

Fig. 1. Overlay Structure 
C. I/0 Units

\begin{tabular}{|c|c|c|c|}
\hline Unit & Use & Record Lenath & $\begin{array}{l}\text { Number of } \\
\text { Records }\end{array}$ \\
\hline $\mathbf{I}$ & PMANG Record & 623 & 1 \\
\hline 2 & Repository & a & \\
\hline 5 & Input & & \\
\hline 6 & Output & & \\
\hline 8 & MATXS Interface & b & \\
\hline 9 & $\phi^{\star} \phi$ File & ITL*IGH & $1+N Z * I S C T$ \\
\hline 10 & DA, Angular & $(N O A+1)(I M)$ & $2^{\star} I G M$ \\
\hline 11 & DA, Moment & (IGM*IM) & 2*LMOM1 \\
\hline 12 & DA, Bilinear Sources & IM & IGM*LMON] \\
\hline 13 & $D A, \phi^{\star} \phi$ Preparation & ITL*IGM & $H Z * L M O M I$ \\
\hline 14 & Scattering Blocks & c & NSR*ISCT \\
\hline 15 & I0 Cross Sections & IGM & NID \\
\hline 23 & SENPRO Interface & IGM & NP \\
\hline & In Source Ex & tion Path & \\
\hline 31 & Scratch (Unformatted) & $2 \star I M * I G M$ & LMOMI \\
\hline 32 & Scratch (Unformatted) & $2 \star I M \star I G M$ & LMOMI \\
\hline 33 & Scratch (Unformatted) & $2 \star I M \star I G M$ & LMORII \\
\hline 34 & Scratch (Unformatted) & $2 * I M * I G M$ & LAOA1 \\
\hline 35 & Scratch (Unformatted) & $2 \star I H \star I G M$ & $2 *$ LMOM1 \\
\hline & In Sensitivity & ecution Path & - \\
\hline 31 & Scratch (Unformatted) & $I T L * I G M$ & ISCT \\
\hline 32 & Scratch (Unformatted) & $I T L * I G M$ & ISCT \\
\hline 33 & Scratch (Unformatted) & $I T L * I G M$ & ISCT \\
\hline 34 & Scratch (Unformatted) & ITL*IGM & ISCT \\
\hline
\end{tabular}




\begin{tabular}{|c|c|c|c|}
\hline Unit & Use & Record Length & $\begin{array}{l}\text { iumber of } \\
\text { Records }\end{array}$ \\
\hline 35 & Scratch (Unformatted) & ITL* IGM & ISCT \\
\hline 41 & Scratch (Formatted) & 80 & NR \\
\hline 42 & Scratch (Formatted) & 80 & NR \\
\hline
\end{tabular}

a. Problem-Dependent,

Angular flux record length $=(\mathrm{NOA}+1)(\mathrm{IM}+1)$,

Number of records $=$ IGM,

Source record length $=$ IM* LMOMI,

Number of records $=$ IGM.

There will be fluxes and sources for each response in the study.

b. Use a tape; if it is desirable to set up disk space, study the problem and the MATXS fomat and estimate space requirements.

c. For upscatter problems, the length of a scattering block is IGM2.

For downscatter only problems, length $=\frac{I C M^{2}}{2}+I G r$.
NOA - number of angles. NSR - number of scattering reactions.
$\mathrm{NZ}$ - number of zones. NID - number of 10 reactions.
LMnMI - number of moments + 1. IM - number of space points.
IGM - number of groups. ISCT - scattering order +1 .
ITL - cross-section table NP - total number of profiles. length.
DD Card Preparation for Direct Access (DA) Units

DC cards for direct access units require the entry of a block length and the number of blocks. These should be calculared with sare since inadequate space will kill the run. Large block lengths use core storage; small block lengths increase 1/0's. The procedure is illustrated by example. 


\section{VI! -40}

Rule 1

Length $<32000$ bytes.

\section{Rule 2}

Length must be an even multiple of 16 .

\section{Example}

Determine record length and number of records for unit 13 when

$I T L=131, I G H=126, N Z=3$, MOMI $=4$.

$L=131 * 126=16506$ mords

$L=66024$ bytes

Rule 1 is violated since $66024>32000$.

Therefore,

$L l=L / N$ v.here $N$ is large enough for $L l<32000$.

Let $N=6$, then $L 1=66024 / 6=11004$.

Now for rule 2, 11004 is not an even multiple of 16 so set $L 1=1100 \%$.

$L 1=11008$ satisfies both rules 1 and 2 . If $N$ could hare been 1 , then

ihe number of records would $=: 12 * \operatorname{LMOM1}=3 * 4=12$. Since $N=6$, number

of records $=12 \star 6=72$. Increase number of records by $\approx 10 \%$ and on the

DD card for unit 13 enter 11008 for the block size and 82 for the number of blocks.

//G0.FT13F001 DD UNIT=SYSDA,

$/ / \quad \operatorname{SPACE}=(11008,(082)$, , CONTIG, ROUND $)$,

$/ / \quad D C B=(R E C F M=F T, B L F N O=1)$ 
IX. JULIET ERROP 5:OPS

Several error stops are built into JULIET shich abort the calculation when a condition is detected that will invalidate the results. In addition to numbered and unnumbered stop statements, the code also includes two error detecting subroutines (ERRA and CRASH). The unnumbered STOP statements always follow a printed statement indicating the nature of the difficulty.

A. The Unnumbered STOP Statements

Location: Subroutine PMATXS, format statement 93

Message: PMATXS is dimensioned for 1500 materials, this problem has, IIIIIIII

Action: The rixed dimension of IMATMM(1500) in RTATYS must be increased or the problem size reduced. The latter is recommended.

Location: Subroutine RMATXS, format statement 23

Message: Dimensioning problem in RMATXS with variahles associated with N2DB, take action in RMATXS, MIXER, PCHIX, N2D3 = IIIII, MSCAT $=$ JJJJJ.

Action: A material has been read from the MATXS file with a nigher scattering order than anticipated by the program writer. If the scattering order for the problem is less than $P_{21}$, check MATXS for errors; if a scattering order greater than $P_{20}$ is desired, program changes will be required in the routines cited in the message. 


\section{I -42}

Location: Subroutine PMATXS, format statement 76

Message: See PAATXS, increase dimensions of variables associated with NIDR frem 100 to IIIII.

Actio:1: Check MATXS for an error; if none, take the action indicated in the message.

Location: Subroutine RMATXS, format statement 78

Message: Dimension of D exceeded in RMATXS; available space IIIIIIII, required space JJJJJJJJ.

Action: Increase the container array as indicated in the message or reduce problem size.

B. The Numbered STDP Statements

Statement: STOP 11

Location: Subroutine LOCATE

Action: An undefined cross-section operator was requested in either a $725 \$$ or $735 \$$ card. Insure that operator definition input (see Section VI, Block 2 ) is compatible with SOURCE input (see Section VI, Block 4 ).

Statement: STOP 67, STOP 74, STOP 75, STOP 76, STOP 78

Location: Subroutine SOURCE

fction: Increase container array size; for quidance see Secticr. VII, A. (3).

Statement: STOP 111

Location: Subroutine ERRA

Action: See following discussion of ERRA. 
VII -43

C. Subroutine ERRA

Certain types of errors trigger a call to subroutine ERRF which prints a message containing an error STOP number and the subroutine name from which the call to ERRA originated. ERRA is misleading in that, when reference is made to subroutine MAIN, it should read subroutine SOURCE. Subroutine ERRA terminates with a STOP 111 statement.

Message: $\quad$ Error stop 2 in subroutine MAIK

Called from: Subroutine SOURCE

Action: The scattering order requested for the $H_{1}$ operator of a bilinear function exceeds the maximum scattering order allowed for the problem. Check the third entry on the iss card (see Section, VI, B, Block 1) and the second entry of a 7255 card (see Section "I, B. Block 4). The entry on a 7255 card cannot excess the entry on the 1555 card.

Message: $\quad$ Error stop 4 in subroutine MAIN

Called from: Subroutine SOURCE

Action: A nonzero scattering order was requested for the $\mathrm{H}_{2}$ operator of a bilinear function. Correct the third entry of any $735 s$ cards (see Section VI, B, Block 4) found in error.

Message: $\quad$ Error stop 6 in subroutine MAIN

Called from: Subroutine SOURCE

Action: A nonzero scattering order was requested for the numerator of a linear response. Locate and correct the $725 s$ cards in error. 
Hessage: $\quad$ Error stop $g$ in subroutins HAII:

Called from: Subroutine SOJRCE

Action: A mon-zero szatteriny order was requested for the denominator of a linear response. Locate and correct the 7355 cards in error.

Hessage: Error stop 1 in subroutine PLEG

Called fram: Subroutine PLEG

Action: Check 1555 card for input errors relating to the geonetry and quadrature order.

Hessage: $\quad$ Error stop 1 in subroutine P.DIR

Called from: Subroutine ROIR

Action: Check input weights and cosines (see Section VI, E., Block 1).

Message: Error stop 2 in subroutine RDIR

Called from: Subroutine ROIR

Action: Check inpu: weights and cosines (see Section : $1, \Xi .$, Block 1).

Message: $\quad$ Error stop 3 in subroutine ROIR

Called from: Subroutine RDIR

Action: Check all input rolating to the $S_{n}$ quadrature.

D. Subroutine CRASH

This routine is called several times throughout the code to insur. that the size of the container array is-adequate. When an insufficier. size is detected, a self-explanatory message is printed sidtinq: 


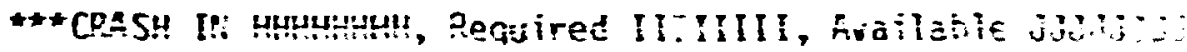

mitere

ب....H is the subroutine name,

I... I is the required container array size,

J...J is the available container array size.

\section{THE MATXS CROSS-SECTIOA FILE}
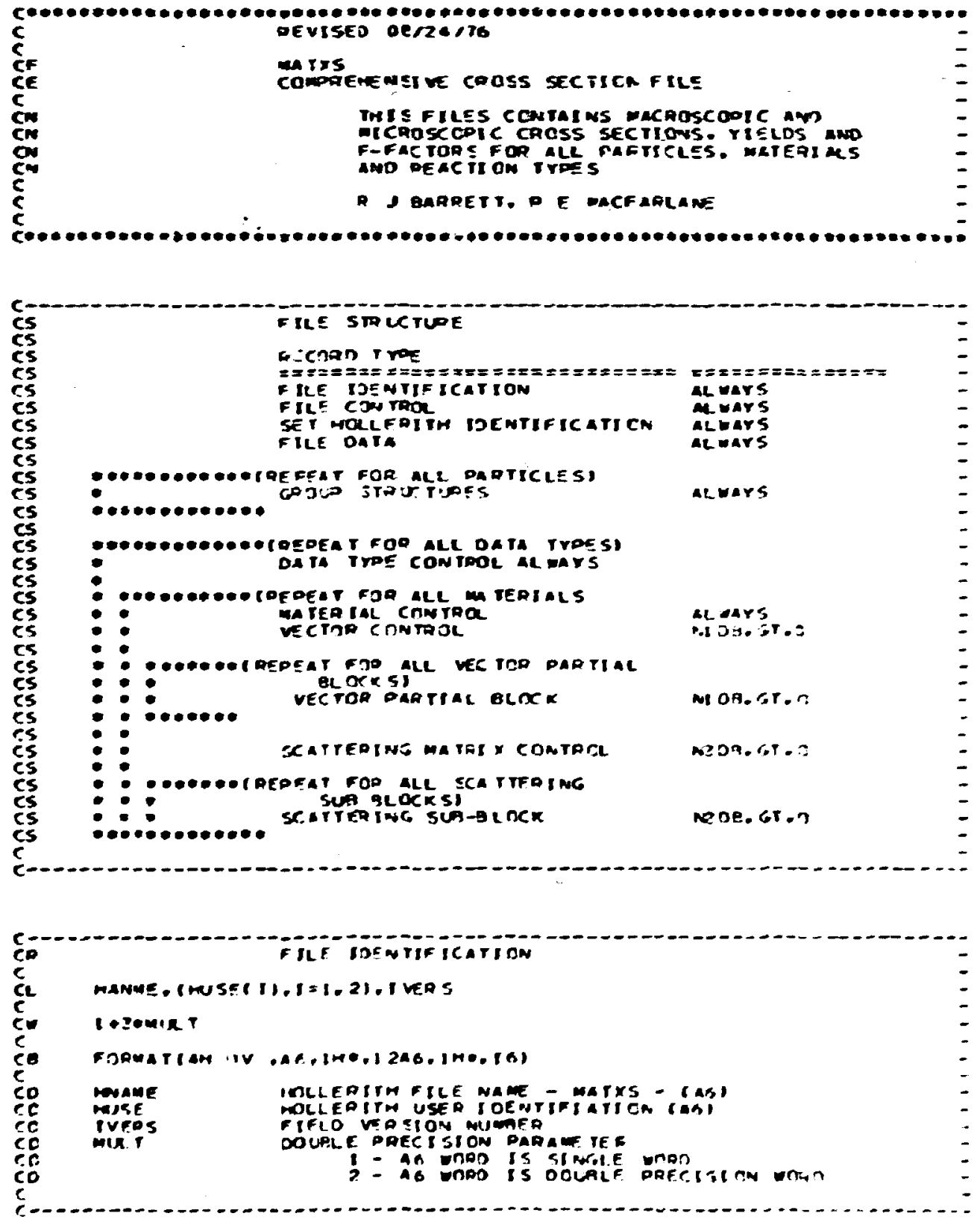

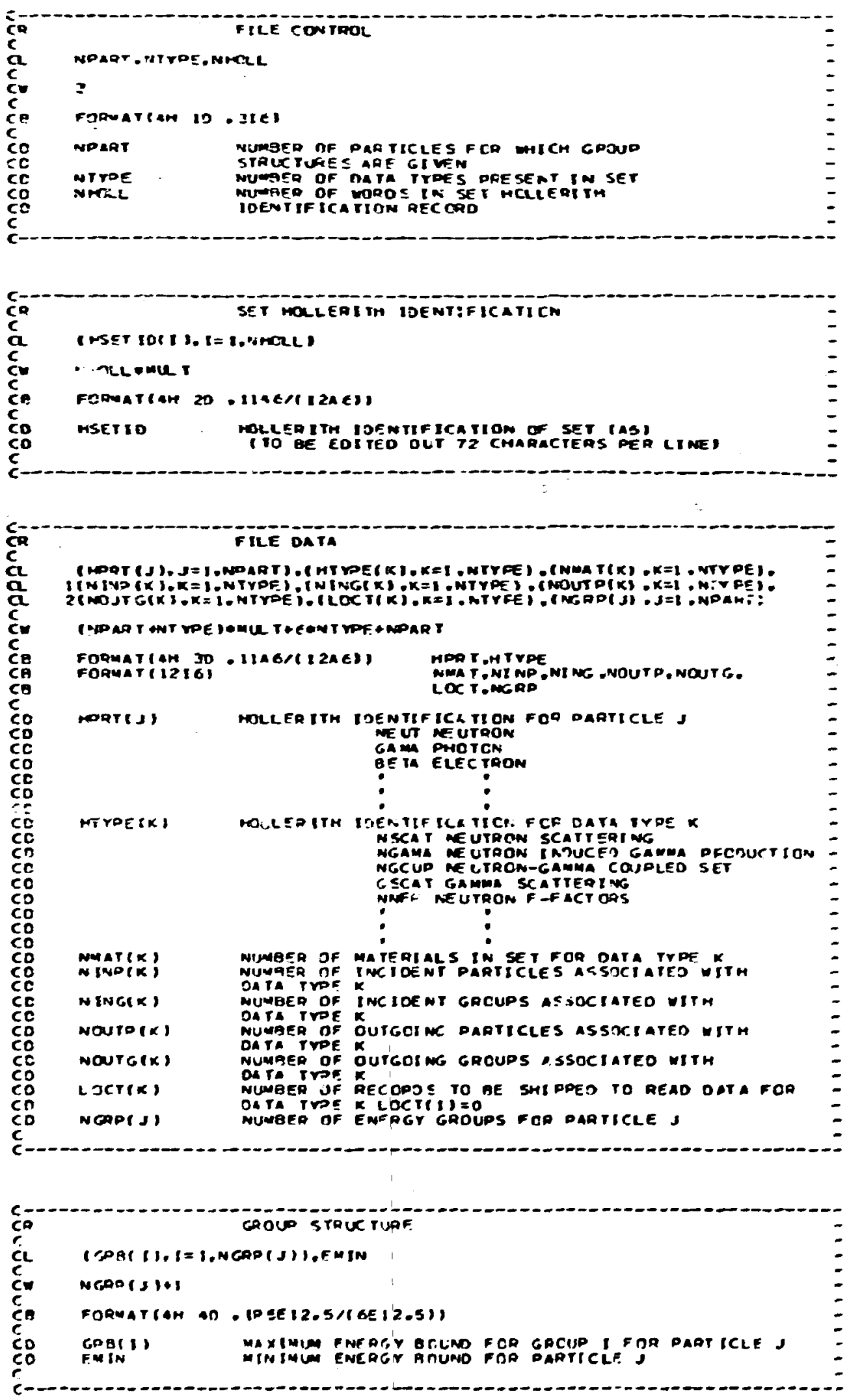

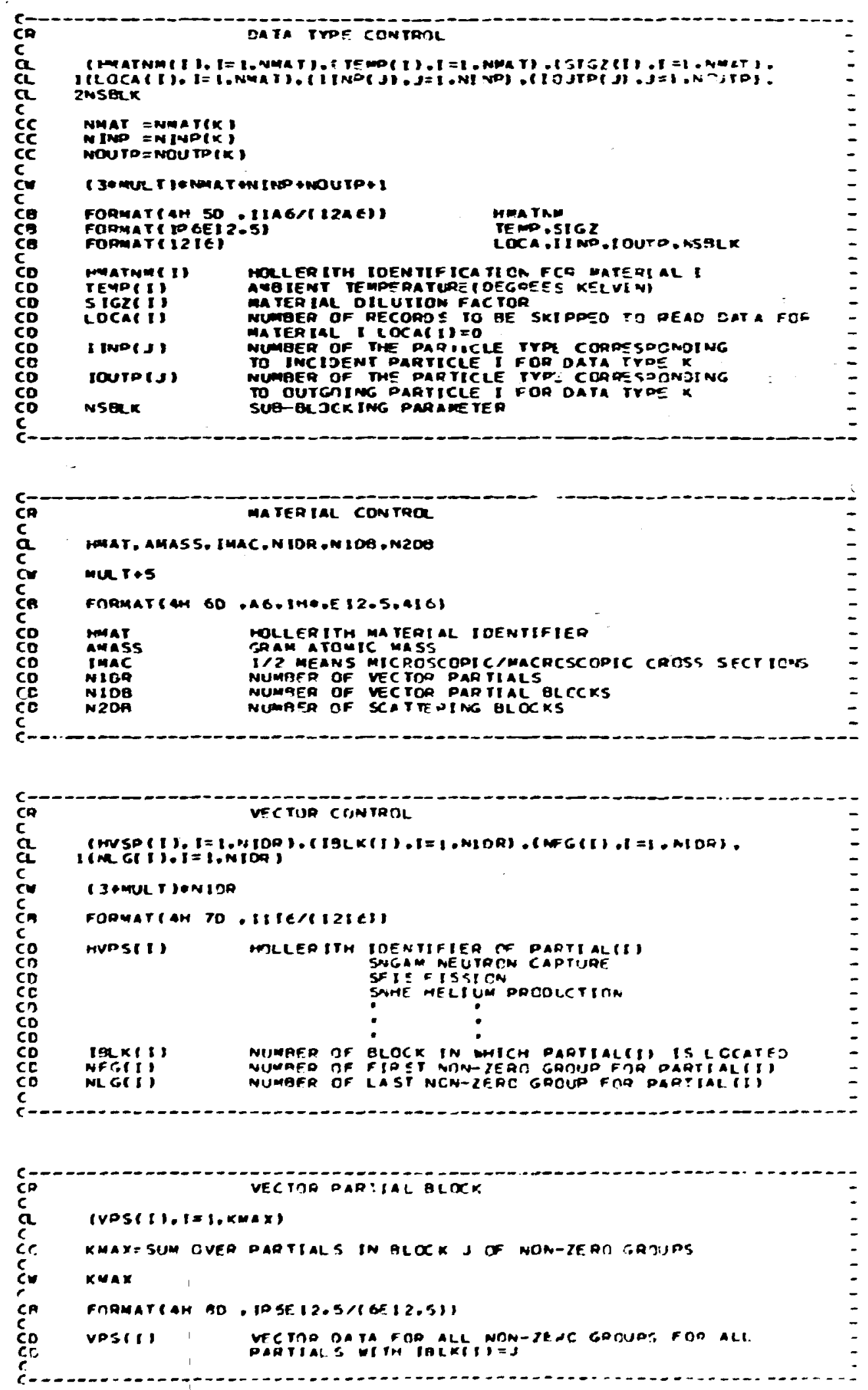

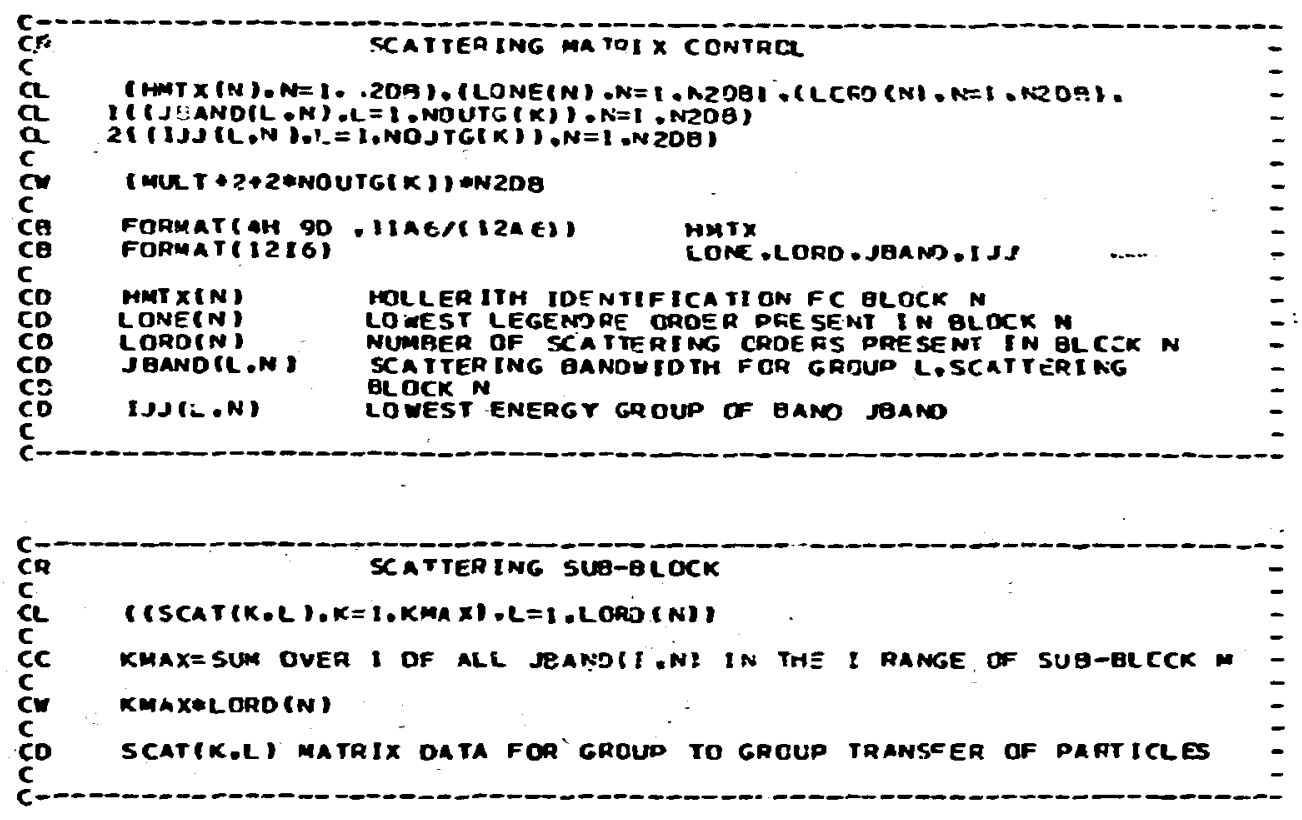

\section{SAMPLE PROBLEM}

This sample problem demonstrates the interaction of the FMANG, FANISN, ANTMX, and JULIET modules in caiculating sensitivity coefficients for the r. and $28 \mathrm{C} / 2.5 \mathrm{~F}$ responses of the $2 P R 6 / 7$ assembly. It al so demonstrates the calculation of generalized sources for a worth response. Cross section requirements, identification schemes, and unit assignments are discussed in tre following comments.

1. A shielded, microscopic, ANISN, cross section file is available on unit 4.

2. A shielded, macroscopic, forward, ANISN, cross section file generated by AXMIX is available on unit 16 .

3. A shielded, macroscopic, adjoint, ANISH cross section file generated by AXMIX is available on unit 17. 
4. The input for a forward FANISN core model of ZPR6/7 has been prepared and the card images placed on unit 38.

5. The following identifiers have been assigned:

IASB $=12$, assembly identifier on the Repository and the SENPRO file for $Z P R 6 / 7$.

IRESP $=21$, response related identifier on the repository for the bilinear, genaralized, adjoint, ?39Pu worth, source and flux.

IRESP $=22$, response related identifier on the Repository for the bilinear, generalized, forward, ${ }^{239} \mathrm{Pu}$ worth, source and flux.

IRESP $=31$, response reiated identifier on the Reporitory for the linear, generalized, adjoint $28 \mathrm{C} / 25 \mathrm{~F}$ ratio, source, flux, and normalization parameters.

IDS $=4$, response identifier on the SENPRO file for $28 \mathrm{C} / 25 \mathrm{~F}$ sensitivity profile.

NTRN $=3$, reaction rate ratio identifier on the SENPRO file for the $28 \mathrm{C} / 25 \mathrm{~F}$ sensitivity profile.

$O P I D=1$, the automatic operator identifier for the mixture of fissionable materials in the ZPR $6 / 7$ core.

OPID $=2$, the automatic operator identifier for

6. The $\phi^{*} \phi$ file for $k$ sensitivity will be placed on unit 9 .

7. The $k$ sensitivity profile will be placed on unit 23 .

8. The $\Gamma_{i}$ file $28 \mathrm{C} / 25 F$ sensitivity will be placed on unit 28 .

9. The $28 \mathrm{C} / 25 \mathrm{~F}$ sensitivity profile will be placed on unit 24 . 
VII -50

10. In the ANISN to MATXS input, note that the group boundaries are all one, 4** Fl.0 T. It is not essential for wis pi oblem that the Mix file contain a realistic group structure since the MATXS file is not being retained as a file of record.

11. In the input to calculate sensitivity profiles for $28 \mathrm{C} / 25 \mathrm{~F}$, note that the group boundaries are all one, $81 * \star \mathrm{Fl} .0 \mathrm{~T}$. However, the group boundaries are specified for the $k$ sensitivity calculation with the 810 (6E11.4) and following cards ending with the T. It is anticipated that the $k$ profiles (unit 23 ) and the $28 \mathrm{C} / 25 \mathrm{~F}$ profiles (unit 24) will be merged to create a single SENPRO file. The group boundaries from unit 23 will be placed on this new SENPRO file. Therefore, a repetition of the group boundaries is unnecessary. 


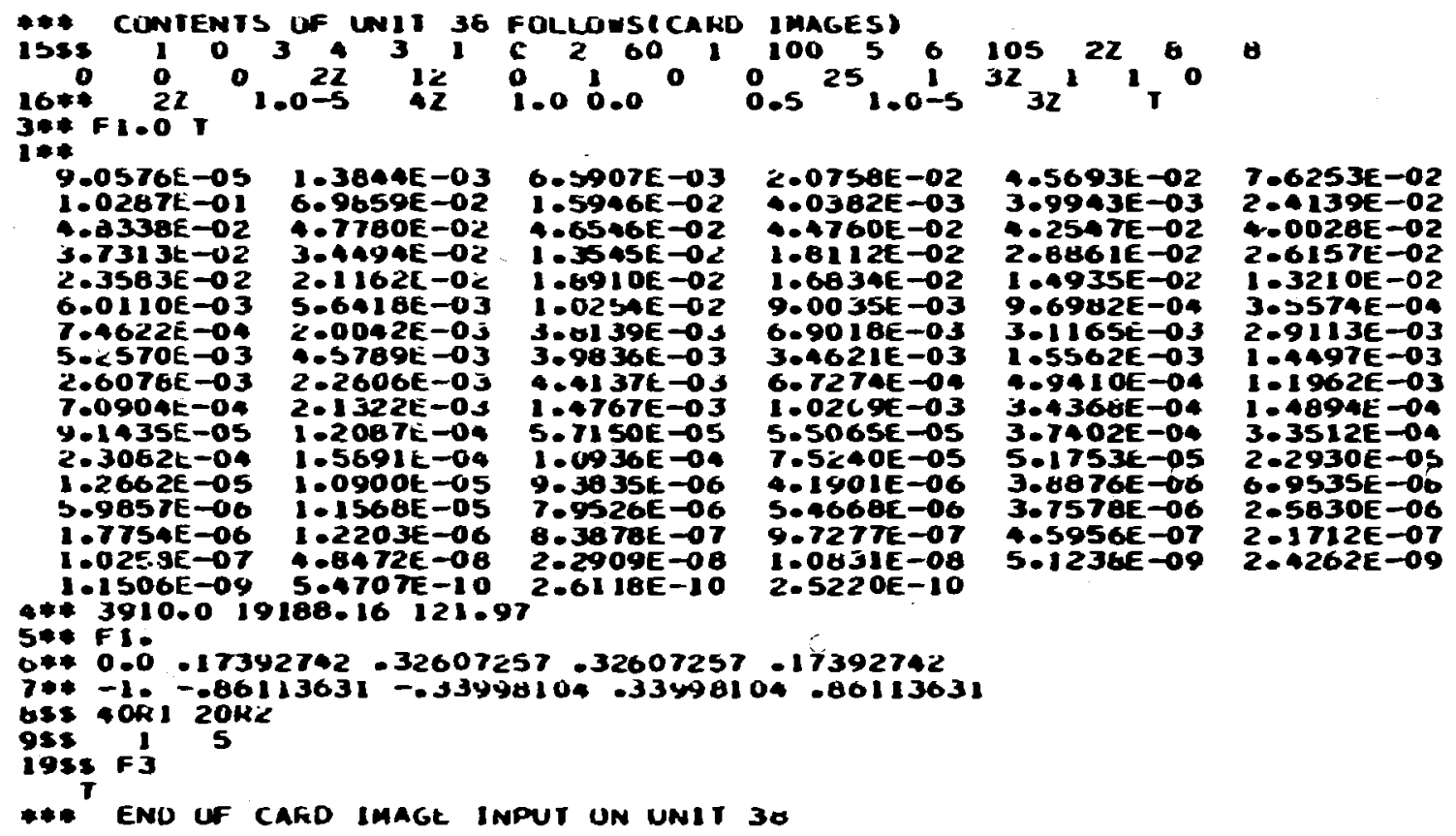

FORSS INPUT

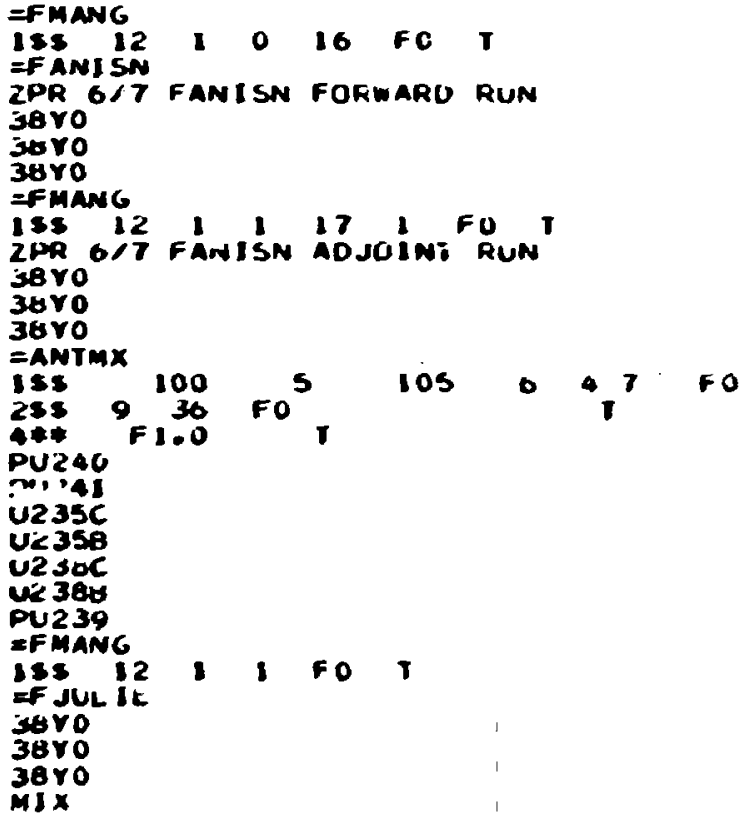




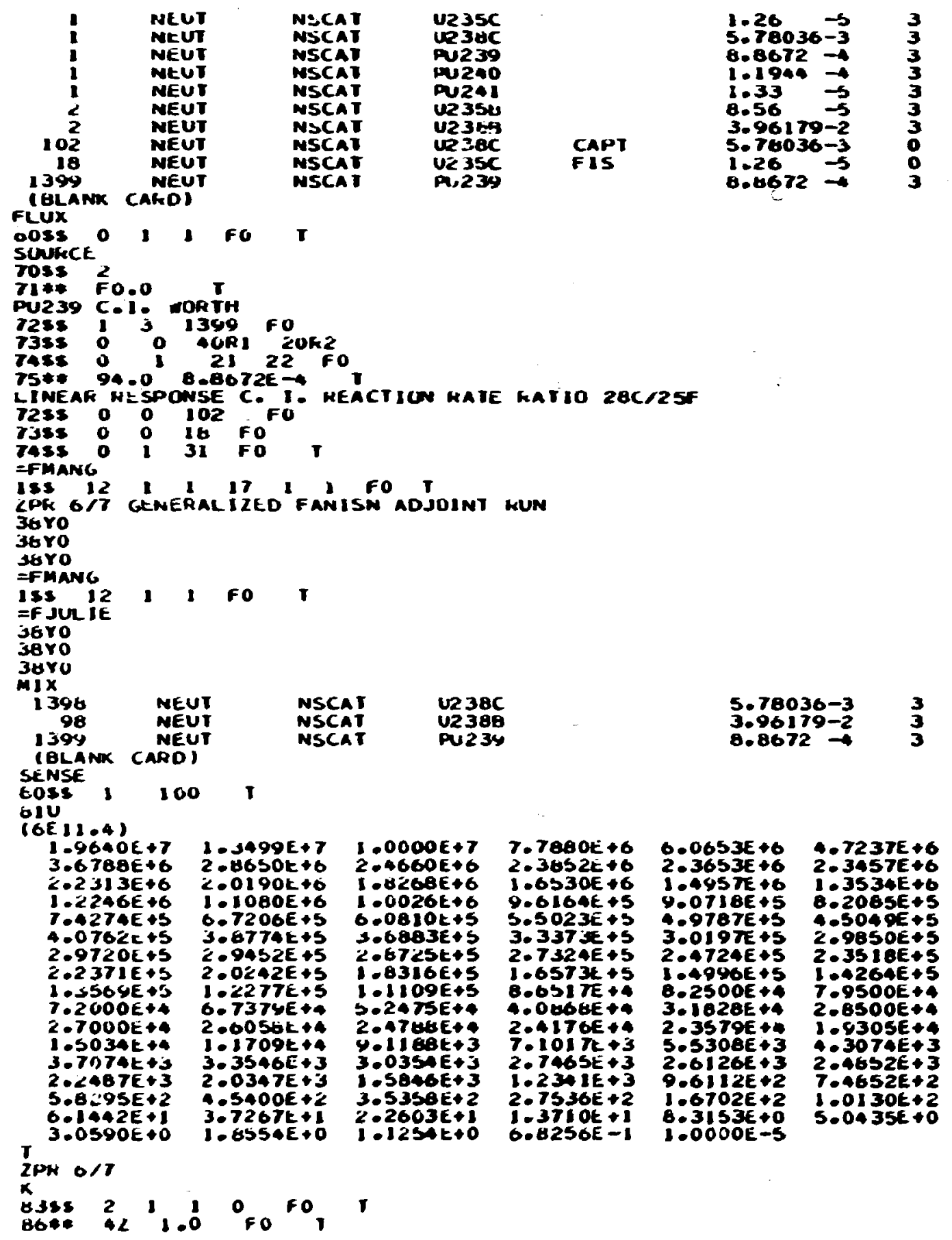




\section{VII -53}

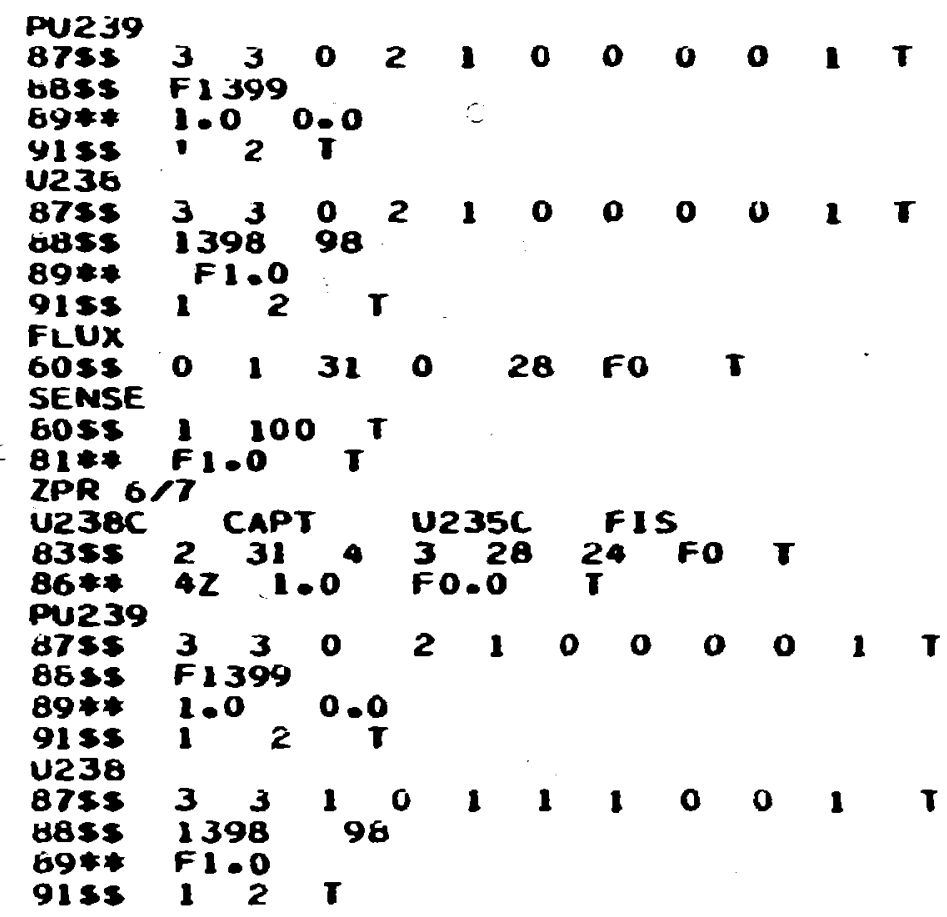




\section{ACKNOWLEDGMENTS}

The authors wish to thark the fo!louing persors for their contributions to the development of JULIET.

- C. R. Heisbin, F. R. Mynatt, and J. H. Marable for support and guidance throughout the project.

- D. E. Bartine and J. V. Pace for consultation on S'iAHLAKE.

- R. L. Childs for consultation on implementing two-dimensionái sensitivity analysis.

- B. L. Broadhead, Yas Seki, E. T. Tonlinson, and V. C. Baker for atd in validating the code.

- Breñda Neeley and Nancy Máuney for the preparation nf numerois drafts.

- Barbara Been for the timely preparation of a preliminary document distributed at the FORSS workshon.

- Cathy 01dham for the design and production of the finished manuscript. 


\section{REFEREMCES}

1. C. R. Keisbin, E. H. Oblow, 3. H. Marable, R. H. Peelle, and

J. L. Lucius, "Application of Sensitivity and Uncertainty liethoosiogy

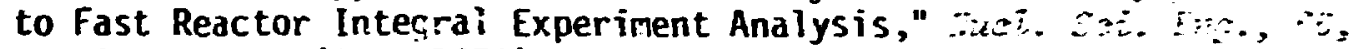
No. 3, 307-333 (June 1978).

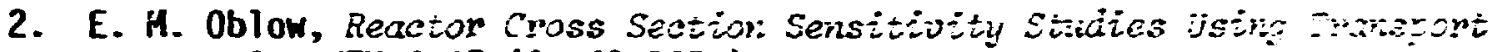
Theory, ORNL/TM-4437 (April 1974).

3. D. E. Bartine, E. H. Oblów, and F. R. Mynatt, SHRHAE - $A$ Canter

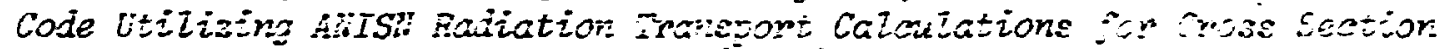
Sensi:ivijy Anal:sis, ORNL/TM-3809 (1973).

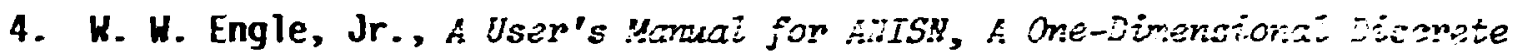
Ordinates Transpori Code witr Enisotropic Scattering, K-1693, Computing Technology Center, Dak Ridge Gaseous Diffusion Plant (1967). Revised by $E$. H. Oblow to perform generalized calculations, see Ref. 2.

5. H. A. Rhoades and F. R. Mynatt, The jot III Two Dimenciorat Discrese OrGïiases Trarspont Cosis, ORNLiTH-4280 (Septenber 1973).

6. D. R. Vondy, T. B. Fowler, and G. W. Cunningham, III,

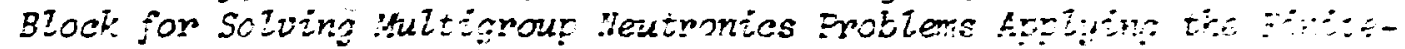

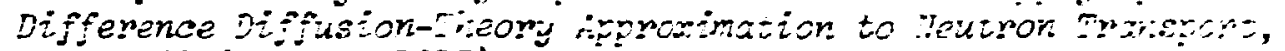
ORNL-5062 (October 1975).

7. J. L. Lucius, C. R. Weisbin, J. H. Marabie, J. D. Drischler,

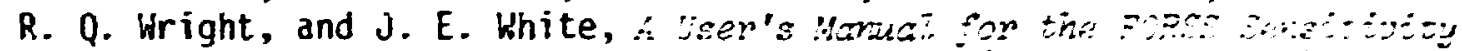

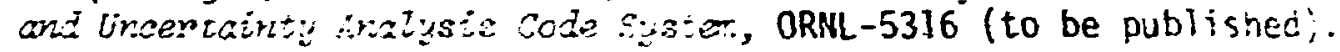

8. R. J. Barrett, R. E. MacFarlane, and R. M. Boicourt, A

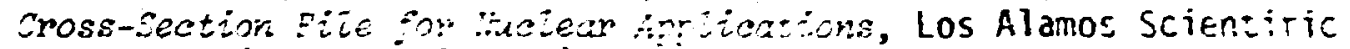
Laboratory (to be published).

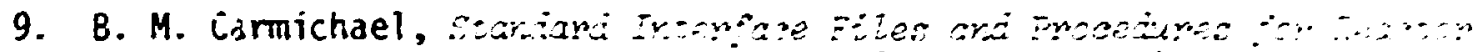
Prisias Codes, Versior. II, LA-5486-MS (February 1974).

10. J. L. Lucius and C. R. Weisbin, "Interface Specifications for Sensitivity Profiles (SENPRO) and Covariance Files (COVERX)," Presentation to the Comittee on Computer Code Coordination, Los Alamos Scientific Laboratory (Hay 1976).

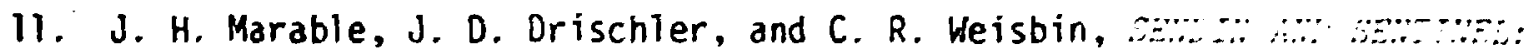

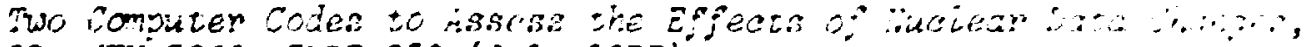
ORNL/TM-5946, ENDF-250 (July 1977).

12. J. D. Drischler, J. H. Marable, and C. R. Weisbin,

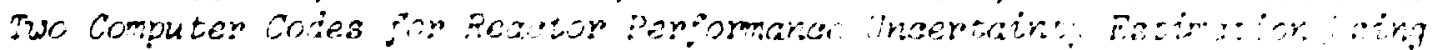

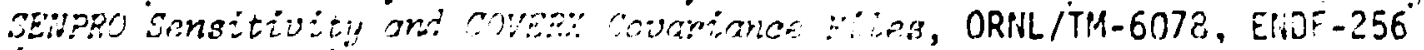
(to be published). 
13. UNCOVER is a revision of the AMARA program permitting the solution of problems with a large number of energy groups. See also. h. Gandini

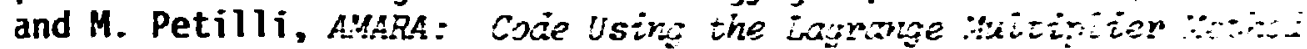
for Muclear Data ficiusment, private comunication, 11 . Salvatores $(1975)$.

14. C. R. Meisbin, J. H. Marable, J. L. Lucil:s, E. M. Oblow, F. R. Myratt, R. W. Peelle, and F. G. Perey, Appiraction of FORSE Sensitiviti: rov

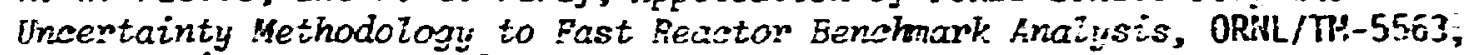
ENDF-236 (December 1976). 
CHAPTER VIII. THE SENPRO SERVICE MODULE

OF THE FORSS SYSTEM

J. L. Lucius 
EHAPTER VIII

Table of Contents

Page

Abstract ....................... v v

Introduction ...................... VIII-3

SENPRO file List ...................... . . VIII-4

Graphic Display ...................... VIII-4

Complete file Plotting. . . . . . . . . . . . VIII-5

Selective Plotting ................. VIII -5

SENPRO File Add/Delete .................... VIII-6

Add option ..................... vIII-6

Delete Option .................... VIII-8

SENPRO File Merge. . . . . . . . . . . . . . . VIII-8

SENOIN-Format Conversion ................ VIII-9

SENPRO file Size ..................... VIII-9

SENPRO file vormalization............... VIII-3

SENPRO file MAT-MT Control Record Fix............. VIII-10

SENPRO NeW Grid ..................... Y YIII-II

SENPRO File Edit/Copy. ................... VIII-II

SENPRO file Sum ................... VIII-I3

SENPRO File Group ..................... VIII-14

References..................... VIII-17

Appendix A. The Format for Standard Interface File SENPRO

for Group-Dependent Sensitivity Coefficients. . . . VIII-19 


\section{ABSTRACT}

The SENPRO Service Module includes twelve execution paths to aid in understanding and using the group-dependent sensitivity coefficients contained in the standard interface file SENPRO. The execution paths provide the following operations on SENPRO file(s).

1. Lists the contents of a SENPRO file.

2. Plcts a SERPRO file.

3. Operates on a SEMPRO file by adding or deleting sets of sensitivity coefficients.

4. Herges two SENRRO files and creates a new file.

5. Changes the mode of a file. Unformatted to formatted and conversely.

6. Lists in order of descending absolute magritude of the trtal sensitivity, the title and total sensiti.ity for eacn profile in the SENPRO file.

7. Renormalizes sensitivity coefficients.

8. Redefines any entry in the 12-word MAT-MT control record.

9. Places the sensitivity coefficient; on a user-supplied group stri.cture.

10. Selectively edits or copies a file.

11. Sums ail profiles in a file and produces a new profile.

12. Replaces or modifies the group structure on a SENPRO file. 


\section{INTRODUCTION}

Sets of sensitivity coefficients are calculated by the JULIET i module of the FORSS ${ }^{2}$ system and placed on a proposed CCCC $^{3}$ file, SENPRO. ${ }^{4}$ Each set is identified by an assembly number, a response number, a material number, a reaction type number, and, if applicable, a reaction rate response number. These sets of coefficier.ts are referred to as sensitivity profiles. If the configuration of interest is a CSWEG $^{5}$ fast reactor benchmark, the CSWEG assigned assembiy number is used throughout FORSS analysis. If the configuration is not a CSWEG fast reactor benchmark, the assignment of the assembly number, and the identification must be consistent throughout FORSS analysis. The response and reaction rate ratio identification numbers currently used are listed in the MAT-MT control record description of the SENPRO file. These will be augmented as required. The material and reaction type identification rumbers are the MAT and $M T$ numbers used in the ENOF/B itles. A complete description of the SENPPO file is in Appendix A.

When the generation of the SENPRO file(s) is complete for a stiddy, a careful review is recommended before it is used. The SENPRO service module is available to aid in this review. If identification or normalization problems are detected, they can often be fuickly corrected by the SENPRO service module. At present, the module has twelve execution paths which perform the following tasks.

1. Lists the contents of a SENPRO file.

2. Flots a SENPRC file.

3. Operates on a SENPRO file by adding or deleting sets uf sensitivity coefficients.

4. Herges two SENPRO files and creates a new iile.

5. Changes the mode of a file. Unformatted to formatted and conversely.

6. Lists in order of descending absolute mag:ilude of the total sensitivity, the title and total sensitivity for aacr. profile in the SENPRO file. 
7. Renormalizes sensitivity coefficients.

8. Redefines any entry in the 12-word MAT-MT control record.

9. Flaces the sensitivity coefficients on a user-supplied gruup structure.

10. Selectively edits or copies a file.

11. Sums all profiles in a file ard froduces a new profile.

12. Replaces or modifies the grouk structure on a SENPRO file.

The user selects the desired execution path by entering one of the following words on a card beginning ir, column 1: LIST, PLOT, ADD, MERGE, DELETE, SEND, SIZE, NORM, FIX, GRID, EDIT, SUM, GROUP. The control words $A D D$ and DELETE access the same programming. Additional input requirements are described for each execution path.

\section{SENPRO FILE LIST}

\section{LIST}

$15 \$$ Integer Parameter [1]

NBIN unit number of the SENPRO file, if NRIN < 0 , only the profile titles will be 1 is ted

$T$

\section{GRAPHIC DISPLAY}

The INTRIGUE-II-C IBM-360 ${ }^{7}$ subroutine package is implemented to provide graphs of sensitivity profiles. Graphs of sensitivity coefficients per unit lethargy vs. energy in MeV are available with the following default characteristics.

1. Logarithmic on both axes.

2. Calcomp pen and irk graphs. 


\section{I I -5}

3. Height $-8^{\prime \prime}$.

4. Hidth $-8 "$ ".

5. ITOPY - $10^{\text {ITOPY }}$ is maximum ordinate value, calculated by code.

6. NCY $=4$ - number of cycles on ordinate.

7. ITOPX=2 - $10^{\text {ITOPX }}$ is maximum absicissa value.

8. $N C X=5$ - number ${ }_{N G}^{f}$ cycles on abscissa.

9. CUT=0.005, if $\sum_{i=1}|S(i)| \leq$ CUT, a graph is not produced.

\section{Complete File Plotting}

Every set of coefficients on the file where $\sum_{i=1}^{N G}|S(i)|>$ CUT will be plucted.

\section{Input Data}

PLOT

$1 \$ \$$ Integer Parameters [2]

IPRO - unit number of file

N - 0 complete file plotting

$>0$ number of coefficients selected for plotting

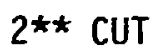

J\$ NCY NCX

$4 \$ \$$ ITOPY 'ITOPX

$5^{* \star}$ WIDTH $^{\dagger}$ HEIGHT

$T$

All of these cards change defau,t characteristics, if the default is acceptable, omit. the card.

\section{Selective Plotting}

The user may select the profiles for plotting. If $N>0$, prepare $6 \$ \$$ card. The $6 \$ \$$ entries identify the profiles with information from the MAT-MT control record.

$\overline{T_{\text {Reducing }}}$ the plot width will result in a disasior. 


\section{VIII-6}

$6 \$ \$$ Integer Parameters [5*N]

IASB - assembly identification

IRESP - response identification

MATID - material identification

MT - reaction type identification

NTRN - reaction rate ratio identification, enter 0 if not applicable

-

- $\quad$ c

Sequence is repeated for each graph requested

$\mathbf{T}$

\section{SENPRO FILE ADD/DELETE}

This execution path perforims three functions. Profiles may be added to the file from card input. Profiles may be deleted from the file. If no profiles are added or deleted, the file is copied without change.

ADD

$1 \$ \$$ Integer Paranti.ers [4]

HOLD - unit number if existing SENPRO file

NEW - urit number of new SENPRO file

NADD - the number of profiles to be added (may be 0 )

NDL - the number of profiles to be deleted (may be 0 )

T

ADD Option

If $N A D D>0$, enter the $2 \$ \$$ array

$2 \$ \$$ Integer Parameters [12]. The MAT-MT contrs,] record of the SENPRO file 


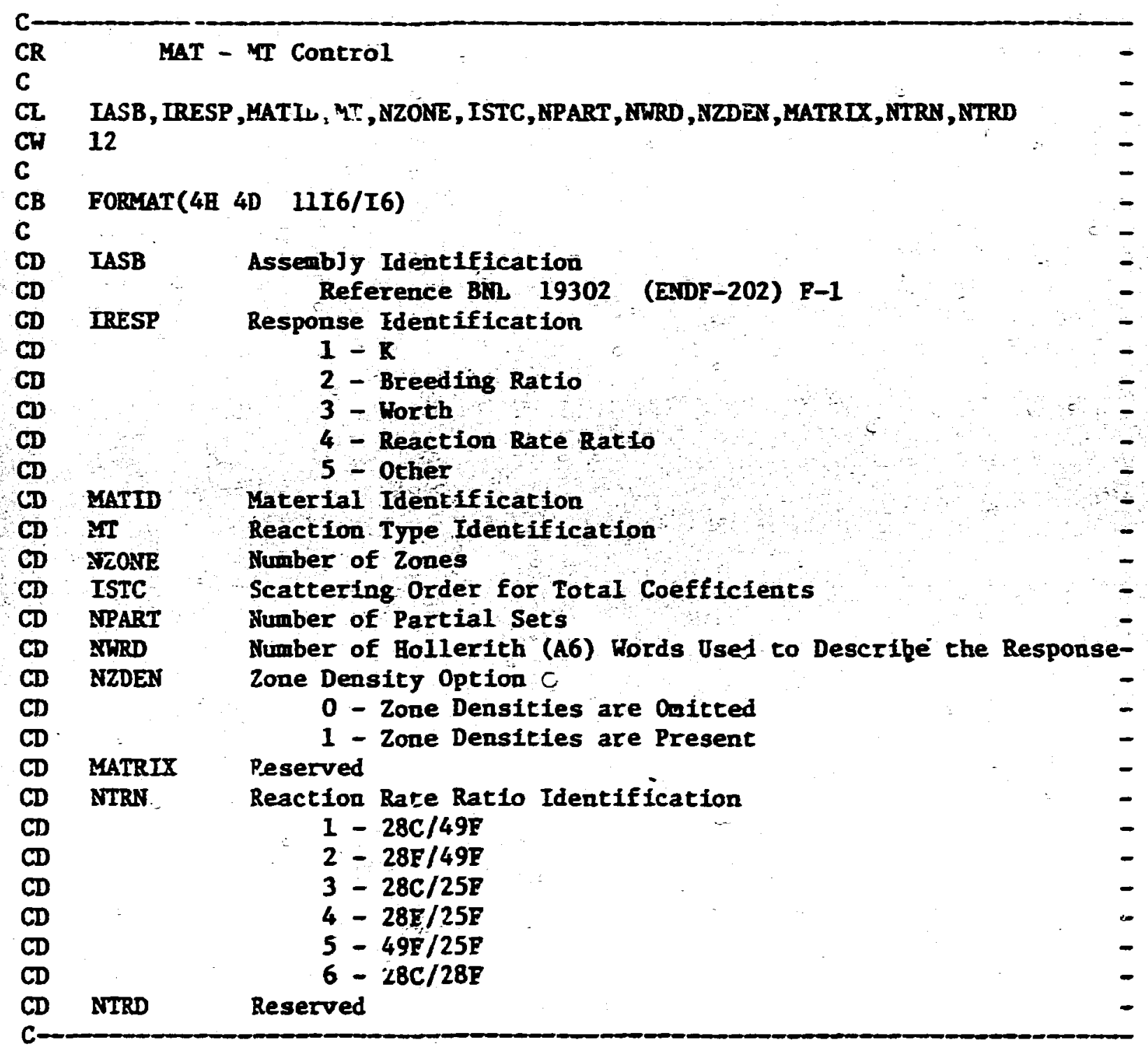


VIII-8

Hollerith response description, FORMAT (11A6)

Input the number of cards needed to define MHRD (A6) words

Hollerith title, 1 card, FOPMAT (11A6)

\section{SENSITIVITY COEFFICIENTS, FORMAT (6E12.4)}

Repeat the sequence until NADD profiles are described.

\section{DELETE Option}

If $\mathrm{NDL}>0$, enter the $3 \$ \$$ array

3\$ Integer Parameters [5*NDL]

IASB - assembly identification

IRESP - response identification

MATID - material identification

MT - reaction type identification

NTRN - reaction rate ratio identification

Sequence is repeated for each profile to be deleted.

T

If NADD $=0$ and NDL $=0$, the SENPRO file on unit NOLD is copied without change to unit NEW.

\section{SENPRO FILE MERGE}

This execution path merges two existing SENPRO files and creates a third file containing all of the profiles on the original files. The files to be merged must have identical group structures. MERGE

$1 \$ \$$ Integer parameters [3]

N1 unit number of a SENPRO fi?e

N2 unit number of a SENPRO file to be merged

N3 unit number of new SENPRO file 


\section{VIII-9}

\section{SENDIN $^{8}$ - FORNAT CONYERSION}

This execution path implements the SENDIN code which converts the SENPRO file from unformatted (binary) to formatted card images and conversely. SENDIN also lists a SENPRO file.

SEND

$1 \$ \$$ Integer Parameters [4]

NBE Select conversion miode

$=0$ unformatted to formatted

$\neq 0$ formatted to unformatted

NIN unit number of existing SENPRO file (DEFAULT = 23)

NOUT unit number of SENPRO file to be prepared by SENDIN (DEFAULT = 24)

No6 Print option

$\leq 0$ File is printed

$>0$ No print

$T$

\section{SENPRO FILE SIZE}

The total sensitivity of each profile on the file is determined. The profile titles are then listed in descending order as a function of the absolute magnitude of the total sensitivity.

SIZE

$1 \$ \$$ Integer Parameter

NBIN unit number of SEAPRO file

$T$

SENPRO FILE NORMALIZATION

This execution path permits the modification of sensitivity coefficients in a SENPRO file. The following two options are ayailable:

1. Renormalize all coefficients by a constant defir.ed as R1/R2, see $2^{* *}$ card. This is useful for salvag*ng sets of coefficients with an iricorrect $R$.

2. Apply a group-dependent multiplication factor. This is useful 


\section{VIII -10}

for applying disadvantage factors. If a profife title card has no total sensitivity (A) or an incorrect $A, 3^{\star \star}$ Fl.0 input will correct the condition.

NORM

$1 \$$ Integer Parameters [2]

NOLD unit number of old SENPRO file

NEN unit number of new SENPRO file

2** Floating Point Parameters [2]

R1 old normalization

R2 new normalization

$\mathbf{T}$

End of input for option 1

If $R 1=R 2=0$, then option 2 is executed

3** Floating Point Parameters [IGM]

Group-dependent multiplication factors

$T$

SENPRO FILE MAT-MT CONTROL RECORD FIX

This execution path allows any word in the 12-word MAT-MT control record to be redefined.

FIX

$1 \$ \$$ Integer Parameters [3]

NI - oid unit number

N2 - new unit number

NFIX - the number of redefinitions to be made

T

$2 \$$ Integer Parameters [3*NFIX]

IP - position in MATMT record to be redefined

NCV - current value of MAiMT(IP) on the file. If NCV $<-10000$, the current value is not considered and $\operatorname{MATMT}(I P)=$ NOV

NDV - desired value of MATMT(IP) 


\footnotetext{
The 3 parameter sequence is repeated until 3*NFIX entries are complete. T
}

SEMPRO NEN GRID

This execution path places each profile in a file in a user designated group structure. The group structure may be input from cards or retrieved from another SENPRO rile. The redistribution of the sensitivity coefficients on the new grid is done linearly in lethargyGRID

$1 \$ \$$ Integer Parameters [3]

MI - unit number of old SENPRO file

N2 - unit number of new SEMPRO file

NG - selection option

When NG $>0$, NG is the number of groups in the new group structure to be defined on input cards. When $N G<C,|N G|$ is the unit number of a SENPRO file containing the new group structure.

$T$

$2 \star \star$ Group Boundaries [NG+1]

(The $2^{\star \star}$ card is included when NG $>0$ ). Enter values from high energy to low energy in units of eV.

$\mathrm{T}$

\section{SENPRO FILE EDIT/COPY}

This execution path permits selective listing and copying of a SENPRO file. Each profile on a SENPRO file has five identification numbers, assembly, response, material, reaction type and reaction rate ratio. The five identification numbers are located in the following positions of the MATMT record of the SENPRO file.

$\begin{array}{lc}\text { Identifier } & \text { MATMT Position } \\ \text { Assembly } & 1 \\ \text { Response } & 2 \\ \text { Materidl } & 3 \\ \text { Reaction type } & 4 \\ \text { Reaction rate ratio } & 11\end{array}$


The five identifiers and their positions in the MATMT record are used to provide a general edit/copy capability. An edit comand consists of the identifiers ordered by assembly, response, material, reaction type, and reaction rate response. Any identifier may be entered as zero which indicates any value encountered for that identifier qualifies for the EOIT/COPY operation. To complete input requirements, a hierarchy must be defined establishing the order in which the identifiers in the EDIT command will be tested. The hierarchy is defined as a function of the identifiers position in the MATMT record. A default hierarchy of $1,2,3,4,11$ is establ ished by the coding. This default hierarchy may be replaced by data input on the $2 \$ \$$ card.

As each profile is read from the SENPRO file, its five identifiers are compared with the corresponding identifiers in each edit comand. The order in which the identifiers are compared is controlled by the established hierarchy. If equality is found between all of the nonzero identifiers in an edit command and the corresponding identifiers from the MATMT record of the profile, the profile qualifies for the edit/copy operation.

\section{Input Data}

\section{EDIT}

1\$\$ Integer Paraneters [5]

[NI] - unit number of existing SENPRO file, if NI < 0 tr.e edited profiles will be copied with listing omitted

NC - number of edit cormands

NEW - copy option $>0$, the unit number of a SENPRO file to be written containing only those profiles edited.

$=0$, file not written

NSS - unit number of a scratch device, default = 31

NHI - hierarchy definition option $=0$, use default definition $>0$, prepare $2 \$ \$$ card 


\section{VIII-13.}

2\$\$ Integer Parameters [5] (Omit if NHI = 0)

Input the MAT-MT positions 123411 in the order requireo to define an appropriate hierarchy.

T

3\$\$ Integer Parameters [5*NC]

Assembly ID

Response ID

Material ID

Reaction type ID*

Reaction rate ratio ID

Complete NC edit commands

$T$

\section{SENPRO FILE SUM}

This execution path prepares one sensitivity profile by sumning every profile contained in a SENPRO file. Practical application usually involves executing the EDIT/COPY, SUM, and MERGE paths in sequence.

\section{Input Data}

SUM

1\$\$ Integer Parameters [2]

NI - old unit number

N2 - new number

T

2\$\$ Integer Parameters [12]

define the MATMT record for the new profile

T

* ${ }^{M T}=5191$ is a special reaction type identifier which permits any inelastic level and the contiuum to be eligible for EDIT/COPY. 


\section{VIII-14}

Response title card for new profile (FORMAT 11A6)

Profile title card for new profile (FORAAT 8A6)

The program supplies the last 3A6 words of a profile title.

\section{SENPRO FILE GROUP}

This execution path replaces or modifies the group structure on a SERPRO file.

\section{Input Data}

GROUP

$1 \$ \$$ Integer Parameters [2]

KOLD - unit number of existing SENPRO file

NEU - unit number of new SENPRO file

T

$2^{\star \star}$ Group Boundaries or Modifiers [Number of groups +1 ]

T

\section{Conments}

If the first and last entries in the $2^{\star \star}$ array are equal, the contents of the $2^{\star \star}$ array are used to modify the group boundaries on the SENPRO file.

where

$$
\mathrm{GB}_{\mathbf{i}}=\mathrm{GB}_{\mathbf{i}}^{ \pm} \star \mathrm{GBM}_{\mathbf{i}}
$$

$$
\begin{aligned}
& \text { GB* - group boundaries on SENPRO } \\
& \text { GBM - modifiers read in 2** arra." }
\end{aligned}
$$

The intent of this is to provide a capability to change the units of the group boundaries on a SENPRO file. For example, $2^{\star \star} \mathrm{FI} .0 \mathrm{OE}+6 \mathrm{~T}$ would change the units from MeV to EV.

If the first and last entries in the $2^{* *}$ array are not equal, the contents of the $2^{\star \star}$ array replaces the group boundaries on the SENPRO file.

The group boundaries entered in the $2^{\star *}$ array may be in either ascending or descending order. GROUP will insure that the boundaries placed on the new SENPRO are in descending order.

GROUP assumes the number of entries in the $2 * *$ array are equal to the number of group boundartes on the SENPRO file. Since all information 


\section{VIII-15}

about the number of boundaries is taken from the SENPRO file, any indication of an error in the number of entries read in the 2** array is disasterous and the input should be carefully checked. 


\section{REFERENCES}

1. J. L. Lucius, E. M. Oblow, and G. H. Cunningham III, "A User's Guide for the JULIET Module of the FORSS Sensitivity and Uncertainty Analys is Code System," ORNL/TH-6594 (ENDF-275) (February 1979); see also Chapter VII.

2. C. R. Meisbin, J. H. Marable, J. L. Lucius, E. M. Oblow, F. R. Mynatt, R. W. Peelle, and F. G. Perey, ApFizcation of FORSS Sensitivity and Uncertainty Methodology to Fast Reactor Benchmark Analysis, ORNL/TM-5563 (1976).

3. B. M. Carmichael, Standard Interface Piles and Procedures for Reactor Phasics Codes, Version III, LA-5486-MS (February 1974).

4. J. L. Lucius and C. R. Meisbin, "Interface Specifications for Sensitivity Profiles (SERPRO) and Covariance Files (COVERX)," Presentation to the Comaittee on Computer Code Coordination, Los Al amos Scientific Laboratory (May 1976).

5. ENDP 202 Cross Section Evaluation Jorking Group Benchar Specifications, BNL-19302 (ENDF-202), Brookhaven National Laboratory (1974).

5. M. K. Drake, Data Formats and Procedures for the ENDF Neutron CroseSection Library, Brookhaven National Laboratory report BNL-50274 (April 1974 Revision).

7. M. B. Emmett, Intrigue-II-C: An IBM-360 Subroutine Fackage for Makixs Linear, Locarithmic and Semilogarithric Graphs Using Either the ialcome Pen-and-Ink or Cathode-Ray-Tube Plotter, ORNL/TM-3944 (1972).

8. J. H. Marable, J. D. Drischler, and C. R. Meisbin, SENDIN arC SEWTINEL: Two Computer Codes to Assess the Effects of inctear Data Changes, ORNL/TM-5946 (E.VDF-250) (July 1977). 


\section{APPENDIX A}

The Format for Standard Interface file SENPRO for

Group-Dependent Sensitivity Coefficients

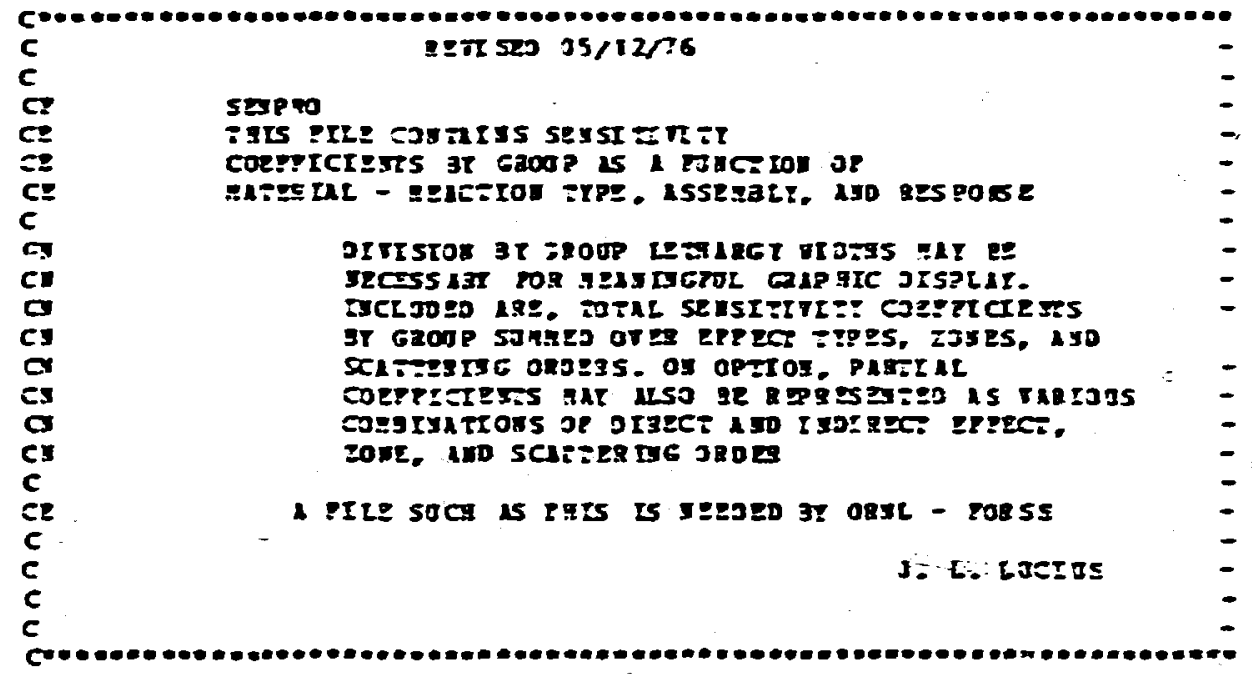

\begin{tabular}{|c|c|c|c|}
\hline$\sigma s$ & 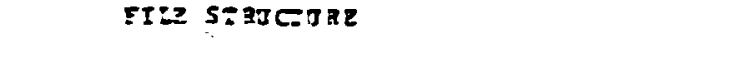 & & $\overline{-}$ \\
\hline cs & 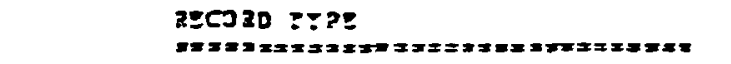 & 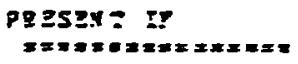 & $\overline{-}$ \\
\hline Cs & IIL IOESTEZCA: TOS & XLEL:S & - \\
\hline $\mathbf{C}$ & PIE =3A?9aL & Lirts & - \\
\hline$=5$ & 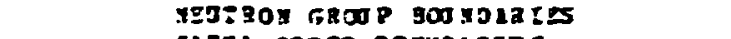 & $9 \times 6 \pi 57.6 \div-0$ & - \\
\hline $\boldsymbol{S}$ & GARTA GROOP BOOSOKzIES & 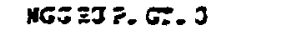 & - \\
\hline es & 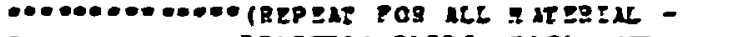 & & - \\
\hline $\boldsymbol{\sigma}$ & 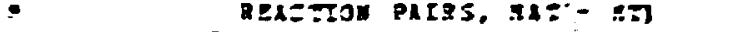 & & - \\
\hline es & 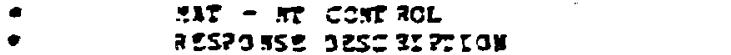 & 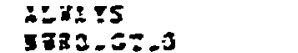 & $\overline{-}$ \\
\hline $\boldsymbol{\sigma}$ & TJYE DESSTEZS & $5202 x .53 .5$ & - \\
\hline 65 & 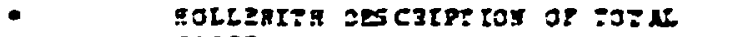 & $1:=x \div \Xi$ & - \\
\hline$=5$ & 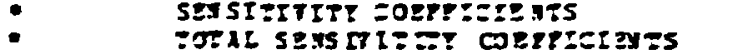 & $15=1 p=$ & $\overline{-}$ \\
\hline$\infty$ & 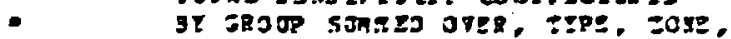 & & - \\
\hline $\mathrm{Cs}$ & 1 10 SCATEESTHG J202R & & - \\
\hline $\boldsymbol{S}$ & - PA2TLLL COYFOL & 1P4:5. 67. & - \\
\hline cs & - 000000000 (REPELT FOB ILL PATEILL & & - \\
\hline$\infty$ & COEFP (2IEST SETSI & & - \\
\hline es & gOLIERTEK OE CATPE IOH OF & IPARE.5Z.J & - \\
\hline es & PAREIAL SEZ & & - \\
\hline$\underset{c s}{c}$ & 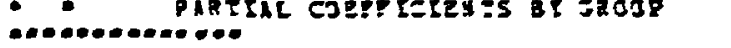 & MFHE:- $5=2$ & $=$ \\
\hline
\end{tabular}

\begin{tabular}{|c|c|c|c|}
\hline$\approx ?$ & PE2 IDEXIFTEA IOH & & - \\
\hline$\zeta$ & & & - \\
\hline$\propto$ & in $\lambda R E, r(\pi S E(I), I=1,2 ;,: 7295$ & & - \\
\hline$c$ & $8 \cdot 3 \cdot 90: 8$ & . & - \\
\hline 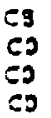 & 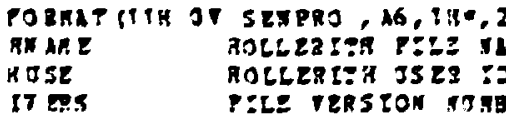 & 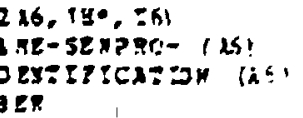 & $\begin{array}{l}\tilde{z} \\
\tilde{z}\end{array}$ \\
\hline c5 & $\begin{array}{l}1=16 \text { IS SLXFLE } \\
2-16 \text { L5 OOTBLE }\end{array}$ & 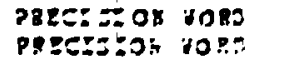 & $\overrightarrow{-}$ \\
\hline
\end{tabular}




\section{II-20}

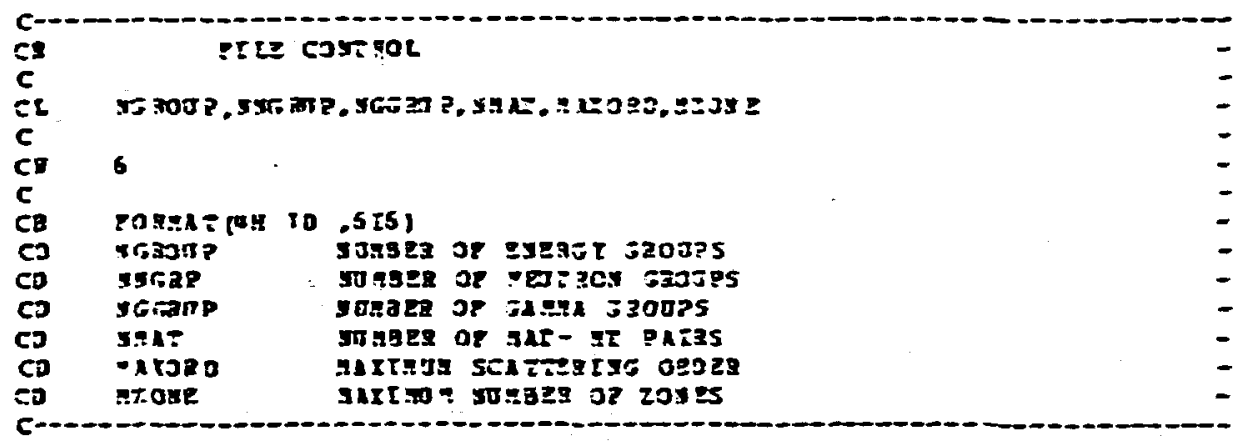

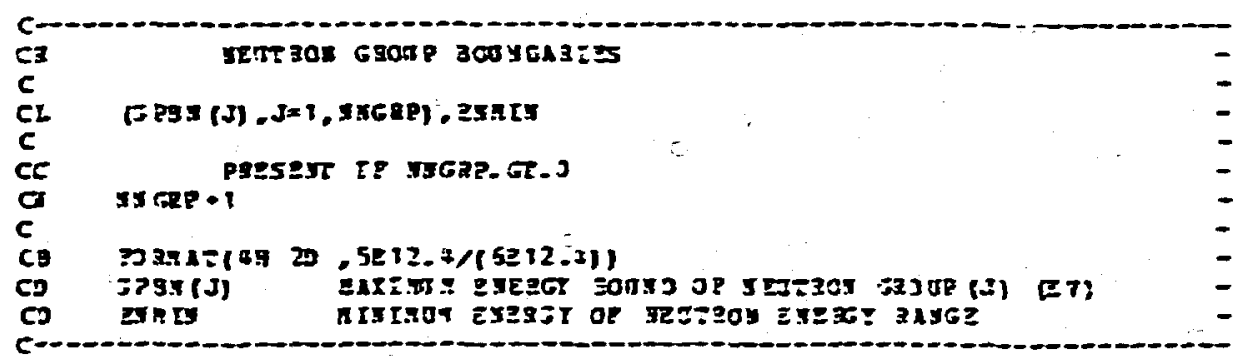

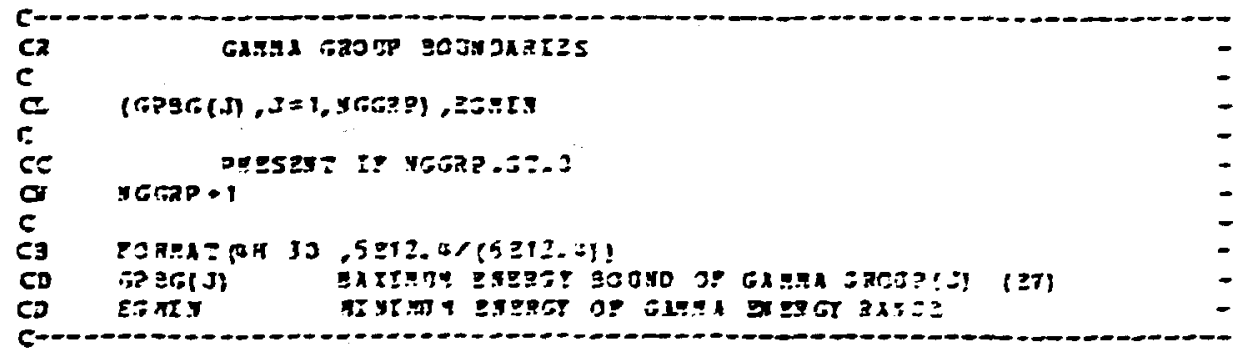

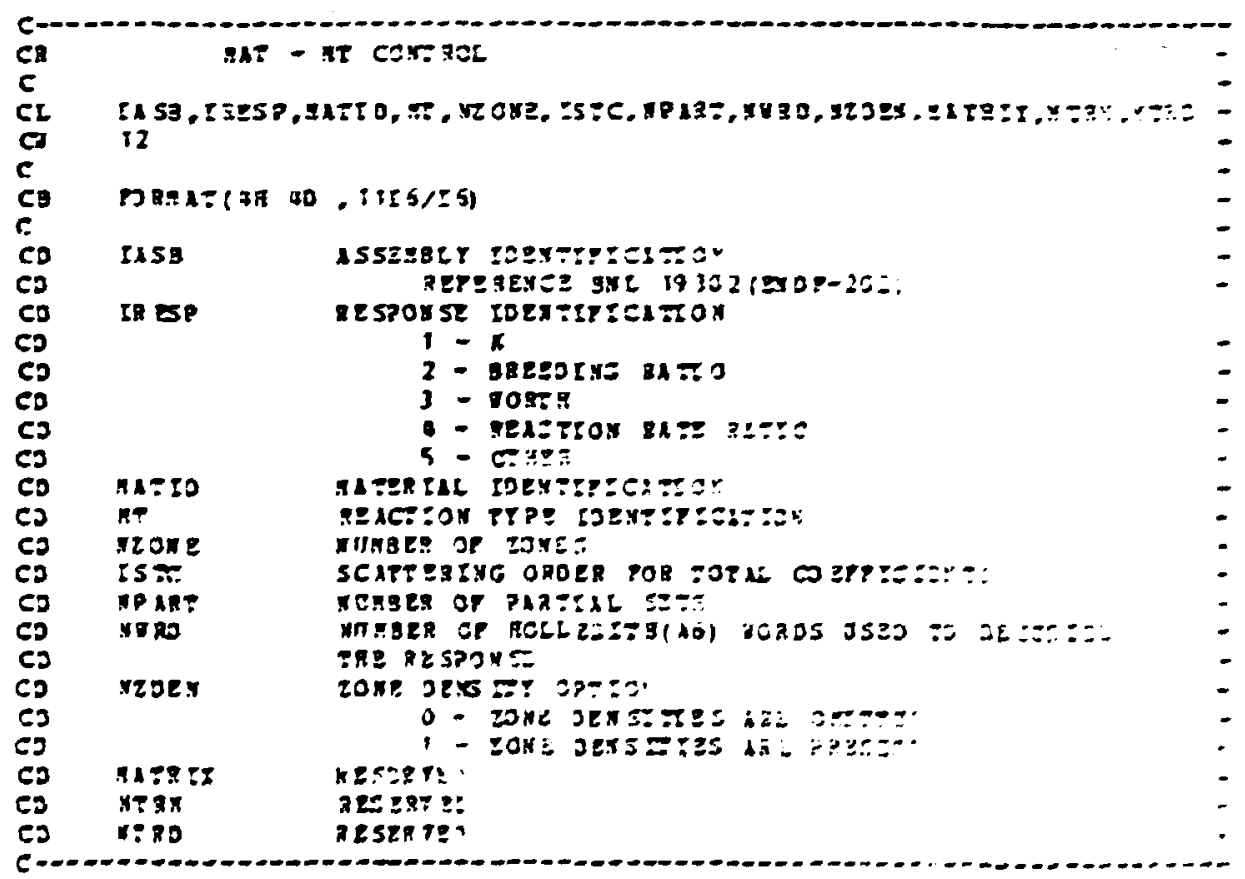




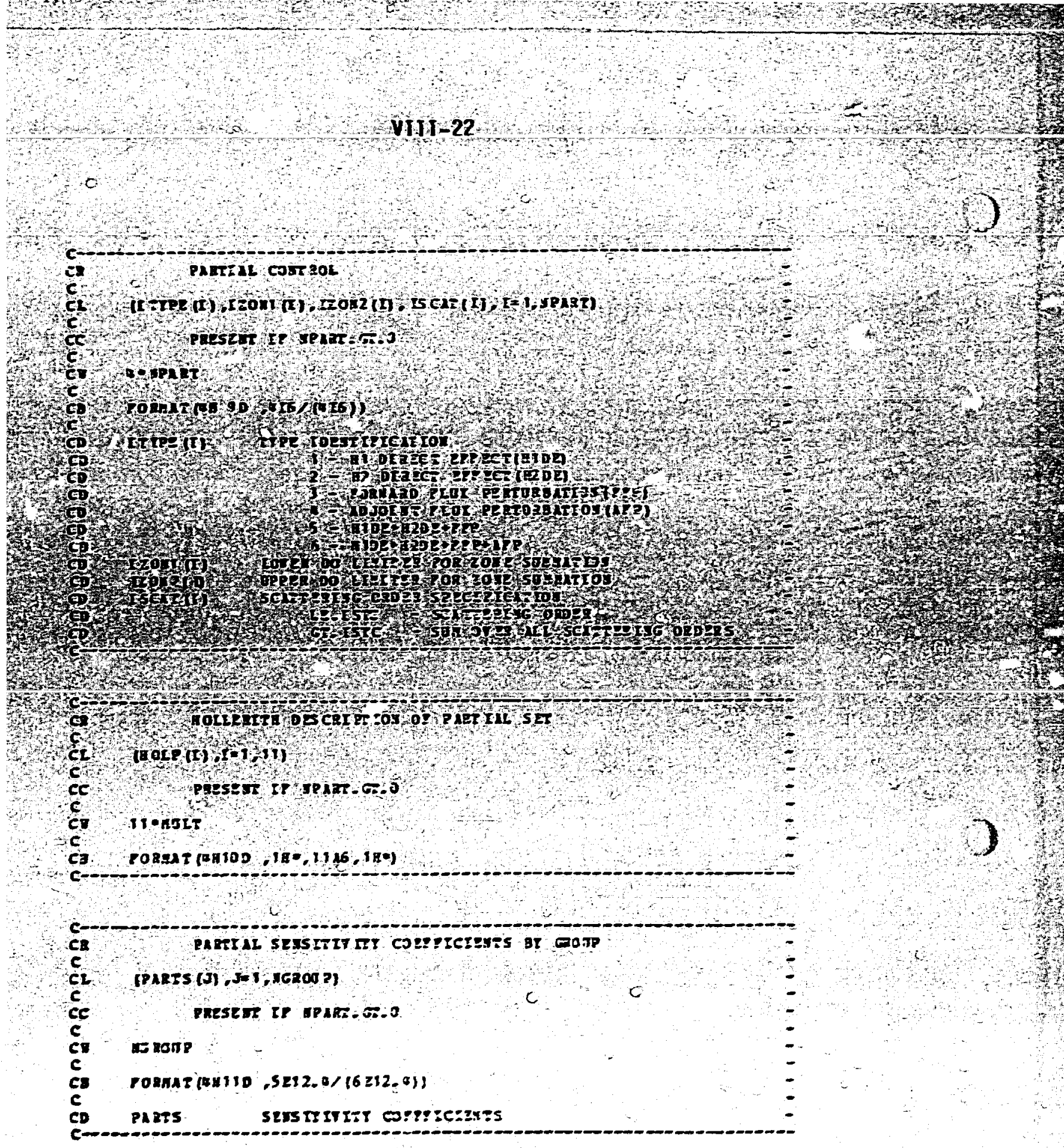


r f

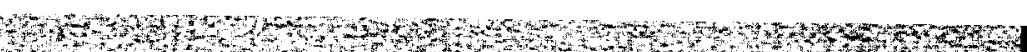



*Origfnally published as ORNL/TM-5946 



\section{ABSTRACT}

A description is given of the computer code SERTINEL, which provides a simple means for finding the effects on calculated reactor and shielding performance parameters due to proposed changes in the cross section data base. This code uses predetermined detailed sensitivity coefficients in SENPRO format, which is described in Appendix A. Knowledge of details of the particular reactor and/or shielding assemblies is not required of the user. Also described is the computer code SENDIK, which converts unformatted (inary) sensitivity files to card image form and vice versa. This is useful for transferring sensitivity files from one installation to another. 


\section{IX-3}

\section{INTRODUC̈CTION}

Sensitivity coefficients for performance parameters of reactor and shielding systems, 1 especially bencharks, have been collected in an interface file ${ }^{2}$ using the SERPRO format. This format was developed according to the standards established by the comittee on Computer codes Coordination (CCCC). 3 The description of the SEAPRO format is given in Appendix A.

In order to use the sensitivity data on a file, it is necessary to have codes which can read such files as well as to perform the required computational manipulations. Such files are ordinarily unformatted (binary) for computational efficiency, but áre not easily read on a different type of computer. To facilitate the transfer of data from one installation to another, the data are converted to card image form. This requires a code shich can translate a binary file to card image form and vice versa.

This report documents two codes. The first, SENDIN, is useful for converting a sensitivity file from binary to card image, for converting from card image to binary, and for obtaining a printed listing of a sensitivity file. The second, SENTINEL, calculates the percent change in a specified response due to given percent changes in specified reaction cross sections over specified energy regions. An edited list of the most significant individual contributions to the response change is also given. 
SERDIN

The program SERDIN copies a sensitivity file in SENPRO format, and in the copying process changes the file representation from binary to card image form or vice versa. In addition, a complete listing or a partial listing of the file is printed to give the user accurate information about the contents of a particular file.

There are two (2) input files:

(1) The SENPRO sensitivity file to be converted and wich is Specified by the FORTRAN data-set reference number NIN (default value 20 ).

(2) The control file which consists of a single card (card image) and which is specified by FORTRAN data-set reference number 5 . This card consists of four integers in (4I5) format: NBE, NIN, MOUT, NoS.

$M B E=0$ conversion is binary to card image.

$\neq 0$ conversion is card image to binary.

NIH = FORTRAN data-set reference number of the input sensitivity file to be conserted (a zero or negative value is replaced by the default value of 20 ).

NOUT = FCRTKAN data-set reference number of the converted output file of sensitivities (a zero or negative value is replaced by a default value of 21 ).

ND6 specifies the printer output FORTRAN data-set reference number and the type of edit - complete or reduced (a zero value of $N D 6$ is replaced by the default value of -61 . 


\section{IX-5}

NP6 > 0 an edit of the entire file is formed on the unit with FORTRAN data-set reference number 196.

$N 96<0$ a reduced edit of the file is formed on the unit with FORTRAN data-set reference number - 1196.

There are two output files (both roferred to above):

(1) The converted sensitivity file with iata-set reference number Nout (default value 21 ).

(2) The file of printer output with data-set reference number specified by 1 is (default value 6 ).

Sample Problem for SENDIN:

The input on unit 5 consists of a single blank card. Unit 20 contains the binary SENPRO fiTe. On unit 21 is a dummy file.

//G9.FT20F001 DO DSN= etc., UNIT= etc.

//GQ.FT2IFOOI DD DUMMY

//GO.FTO5FOO1 DD *

(l blank card)

$f^{\star}$

The blank card could be replaced by the following:

$$
\begin{array}{llll}
0 & 20 & 21 & -6
\end{array}
$$

with the same result.

The printer output on unit 6 produces a reduced edit of the SENPRO file on unit 20 . 
SERTINEL

The program SEKTINEL computes the percentage (or fractional) change in the performance parameter of a given assembly due to specified percentage (or fractional) changes in designated reaction cross sections over a number of energy regions.

The input data consists of:

(1) Parameters defining the performance parameter, the reactor or assembly, the number $N$ of such sensitivities to be included, and the identification for these so that they may be selected from the sensitivity file which is in SENPRO format.

(2) The number of energy regions KMAX and the energy bounds for each such region.

(3) The percentage (or fractional) change in nuclear data for each of the $N$ reactions for wich a sensitivity is specified by (i) above, and over each of the KMAX energy regions specified in (2).

(4) Certain information specifying the printer output data-set reference number (default value 6), and the SENPRO sensitivity file data-set reference number (default value 10).

(5) Information limiting the printout of the most important individual contributions to the resulting change in the performance parameter.

(6) The binary sensitivity file in SENPRO format.

The output consists of:

(1) The input information.

(2) Information selected from the sensitivity file. 
(3) The resulting value of the percentage (or fractional) change in the performance parameter.

(4) An ordered list of the most important individual contributions to the change.

Specifically, the input to SEMTIMEL for one case consists of one card of six numbers and three arrays entered in FIO0 system format. The fIDO system is described in Appendix B. Several stacked cases can be calculated in one run. The following input is required for each case:

one card (format 215, F10, 3I5): N, KHAX, PERCNT, MURB, L'NIT, IQUT

array of energy regions:

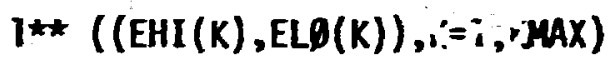

percerit changes:

$3^{\star \star}((\operatorname{PRCENT}(K, N 1), K=1, \operatorname{NMAX}), N 1=1, N)$

sensitivity identifiers:

5\$\$ ( $(I N F Q(I, N I), I=I, 9), M I=1, N)$

FIop teminator: T

$N=$ the number of sensitivity profiles to be included.

KMAX = the number of energy regions to be included.

PERCNT = the minimum magnitude (in percent of the total contribution) of a single (one energy region and one reaction) contribution to be included in a printout of the most important individual contributions.

NuMB $=$ the maximum number of most important contributions to be printed out individually.

LUNIT = the data set reference number of the sensitivity file in SENPRO format (default value $=10$ ).

IQUT $=$ printer output data-set reference number (default value $=6$ ). 
$\mathrm{EHI}(\mathrm{K})=$ an energy bound in $\mathrm{eV}$ for energy region $\mathrm{K}$ (the regions should appear in order of decreasing energy). $E L Q(K)=$ the other bound for region $K$. $\operatorname{PRCEKT}(\mathrm{K}, \mathrm{NI})=$ the percent change in nuclear data associated with sensitivity $\mathrm{NI}$ in energy region $K$.

IR:F $(I, N I) \quad I=i, 9$ consists of nine ident: fiers for the sensitivity profile $\mathrm{N1}$. The nine identifiers in order are:

IASB = the assembly identifier as it appears in the file, or, if the first IASB $(=\operatorname{INF} \theta(1,1))$ is negative, cases are made to include every assembly in the file.

IRESP $=1,2,3,4,5$ according as the response is multiplication factor $k$, breeding ratio, worth, reaction rate ratio, or some other type of response, respectively; or, if the first IRESP $(=\operatorname{INF}(2,1))$ is negative, cases are made to include all responses.

MATID = nuclide identification using ENDF assignments.

MT = reaction identification ușing ENDF assignments.

NTRN $=1,2,3, \ldots$ an acbitrary identification number chosen by the file creator to distinguish different reaction rate or worth responses ( 0 for IRESP $=1$ ).

ITYPE, IZPN1, IZON2, ISCAT refer to partial sensitivities and are set to zero since no partial sensitivities are presently used. 
Sample Problem for SENTINEL:

This problem determines the effect of proposed changes in the fission cross section (MT number 18) of ${ }^{235 \mathrm{U}},{ }^{238} \mathrm{U}$, and ${ }^{239} \mathrm{Pu}$ (MAT numbers 1261, 1262, and 1264 respectively) on the multiplication factor (IRESP=1 and NTRN=0) of each assembly in the file (five), and on the central reaction rate ratio ${ }^{28} \mathrm{c} /{ }^{49} \mathrm{f}$ (IRESP=4 and $\mathrm{NTRN=1}$ ), and on the central reaction rate ratio ${ }^{28} f /{ }^{49} f$ (IRESP $=4$ and $N T R N=2$ ), of the $Z P R-6 / 7$ assembly. (IASB $=5$ is the nianoer assigned this assembly in the SENPRO file reported in Ref. 2.) The proposed changes represent approximate changes in going from ENDF/B-IV to ENDF/B-V. These changes are given in five groups as follows:

\begin{tabular}{cccc}
$\begin{array}{c}\text { Energy Range } \\
(\mathrm{MeV})\end{array}$ & $\begin{array}{c}\text { Change in } \sigma_{f}^{25} \\
\text { (percent) }\end{array}$ & $\begin{array}{c}\text { Change in } \sigma_{f}^{28} \\
\text { (percent) }\end{array}$ & $\begin{array}{c}\text { Change in } \sigma_{f}^{49} \\
\text { (percent }\end{array}$ \\
\cline { 2 - 3 } $3.679-10.000$ & 1.44 & -2.50 & -1.07 \\
$1.353-3.679$ & -0.24 & -3.50 & 0.83 \\
$.498-1.353$ & -2.57 & 12.00 & 1.02 \\
$.183-.498$ & -3.32 & 0.00 & -0.30 \\
$.067-.183$ & -2.13 & 0.00 & -2.13
\end{tabular}

This sample problem uses the fast benchmerks for which sensitivities are reported in reference 2. He assume these sensitivities are available in binary SENFRO format on a unit with data-set reference number 10.

The input can be stacked as three cases. The first searches through all the assemblies in the file (since INFO $(1,1)$ is negative) and manufactures a case for each assembly found. For these we limit to ten (NUMB = 10) the number of individual largest contributions to be printed out, regardless of magnitude. 
The second of the three input cases finds the change in the central reaction-rate ratio ${ }^{29} \mathrm{c} /{ }^{49} \mathrm{f}$ in $2 \mathrm{PR}-6 / 7$ and prints out the largest individual contributions, each of which is not less than one percent (PERCNT $=1.0$ ) of the total calculated change. Not more than five such contributions will be printed out.

The third input case cafculates the resulting change in the ${ }^{28} \mathrm{c} /{ }^{49} \mathrm{f}$ ratio in $2 P R-6 / 7$ and prints out (not more than six) individual contributions greater than four gercent of the total.

The input data files are as follows:

$/ / G D . F T 1 O F O 01$ DD DSN=etc. ,UNIT=VQL= etc.

//GP.FT05F001 DD *
35
$0.0 \quad 10$

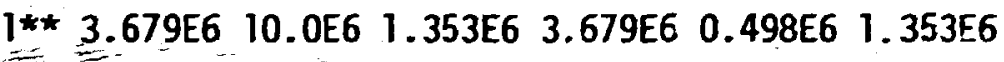

0.498E6 2R0. I83E6 0.067E6

$3 * \star 1.44-.24-2.57-3.32-2.13$

$-2.50-3.5012 .002 Z$

$\begin{array}{lllll}-1.07 & 0.83 & 1.02 & -0.0 & -2.13\end{array}$

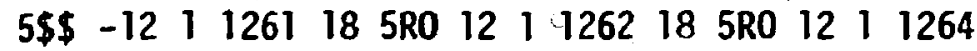

$185 R O T$

$\begin{array}{llll}3 & 5 & 1.0 & 5\end{array}$

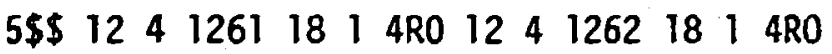

$\begin{array}{lllll}12 & 4 & 126418 \quad \mathrm{ARO} T\end{array}$

$\begin{array}{llll}3 & 5 & 4.0 & 6\end{array}$

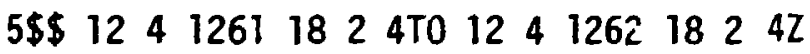

$12412641824 R 0 T$

The output prints a warning message to note whenever a sensitivity is missing in the file and, hence, is not included in the calculation. Note that since the dimensions $N$ and KMAX are constant the order of the reactions (MAT-MT pairs) remains fixed and that the energy regions (1** array) and the assigned percentages (3** array) remain fixed in storage location as well as in value. Therefore, the FIOO $7^{\star *}$ and $3^{* \star}$ arrays need not be read in again after the first case. 


$$
2 x-11
$$

REFERENCES

1. C. R. Heisbin, J. H. Marable, J. L. Lucius, E. M. Oblow, F. R. Mynatt, R. H. Peelle, and F. G. Perey, "Application of FORSS Sensitivity and Uncertainty Methodology to Fast Reactor Benchmark Analysis," : ORN $(7)-5563(7976)$.

2. J. H. Marable, J. L. Lucius, and C. R. Heisbin, "Compilation of Sensitivity Profiles for Several CSEWG Fast Reactur Benchuarks," Oak Ridge National Laboratory report ORNL-5262 (ENDF-234) (March 1977).

3. B. M. rimichael, "Standard Interface Files and Proceusures for Reactor Physic: Codes, Version III," Los Alamos Sciantific Laboratory report LA-5486-MS (February 1974). 


\section{IX-13}

APPENDIX A

The Format for Standard Interface File SEMPRO for Group-Dependent Sensitivity Coefficients 


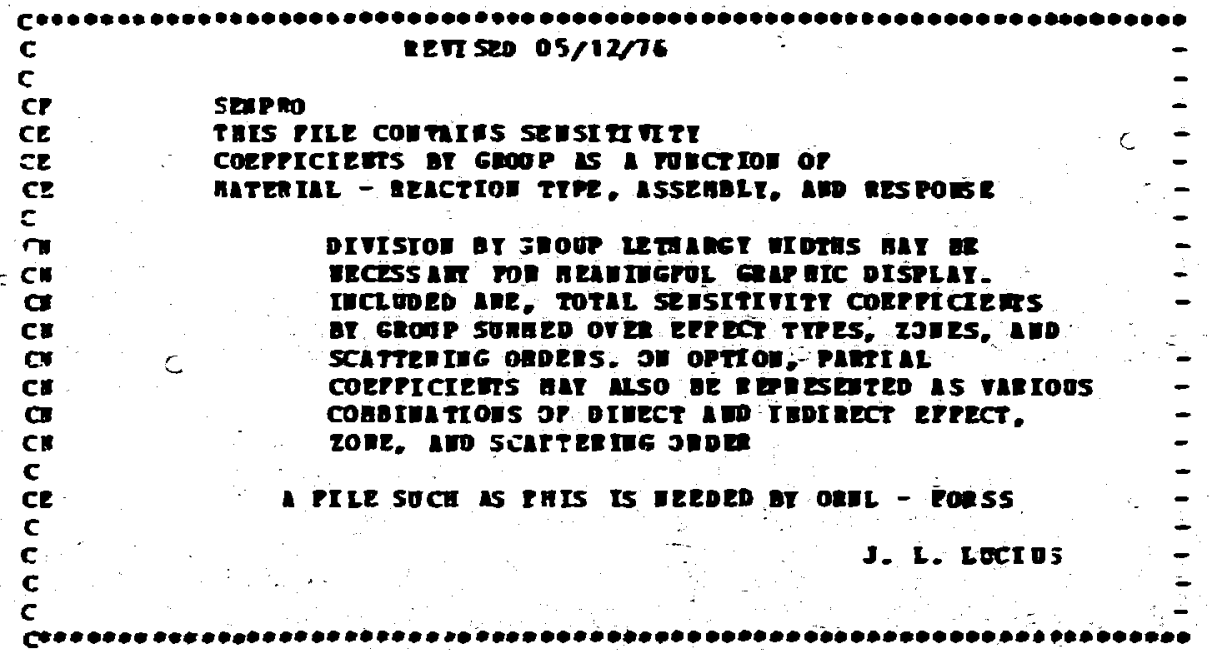

$\Rightarrow$

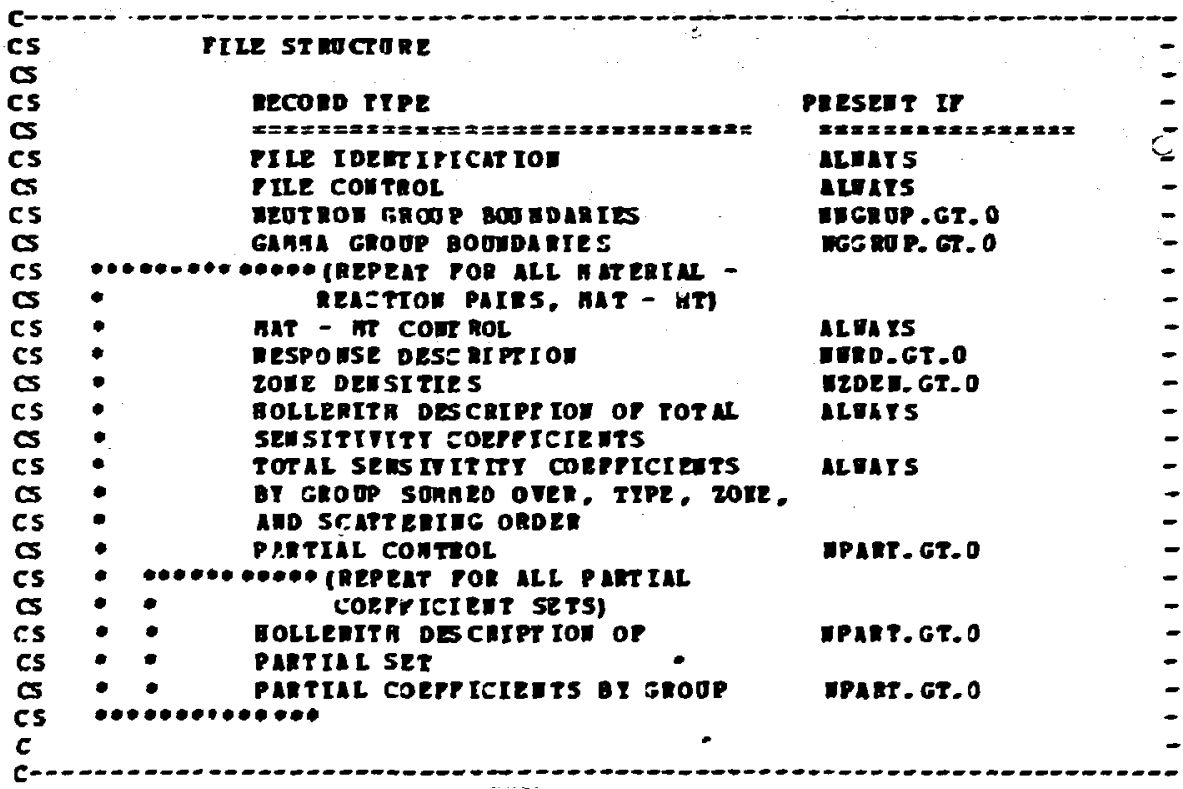

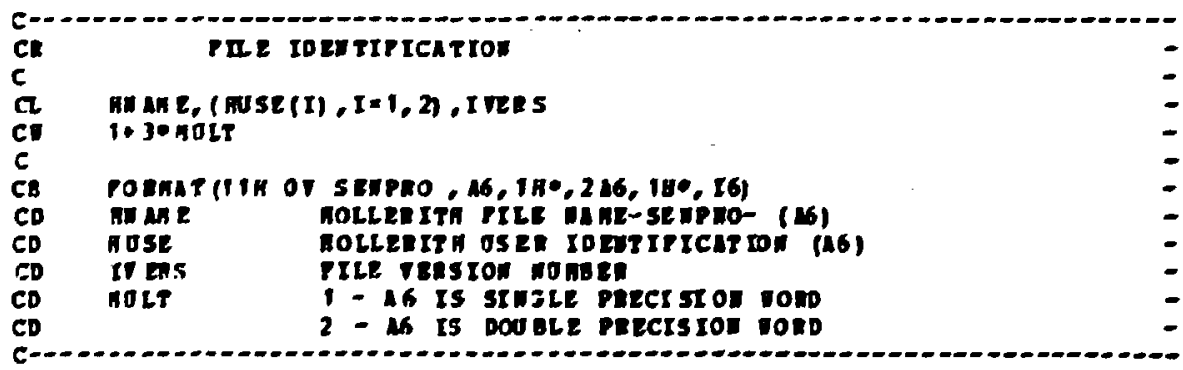




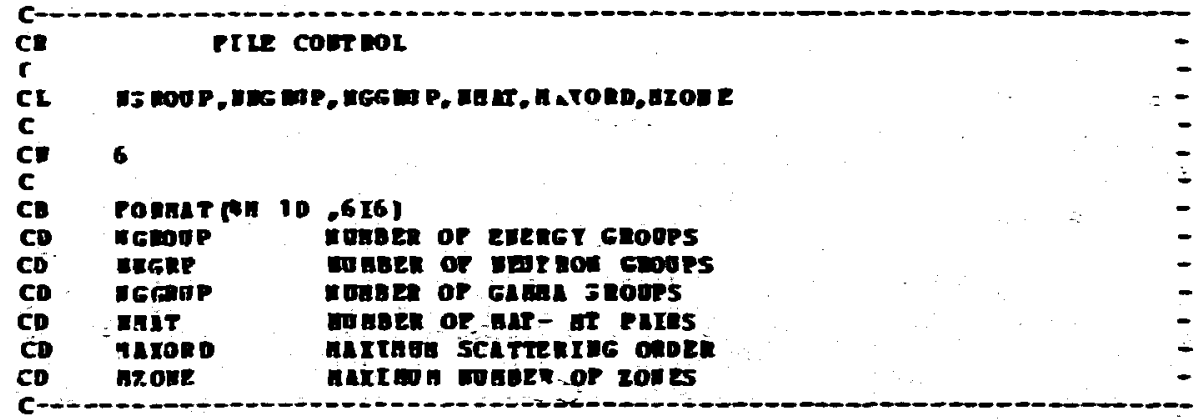

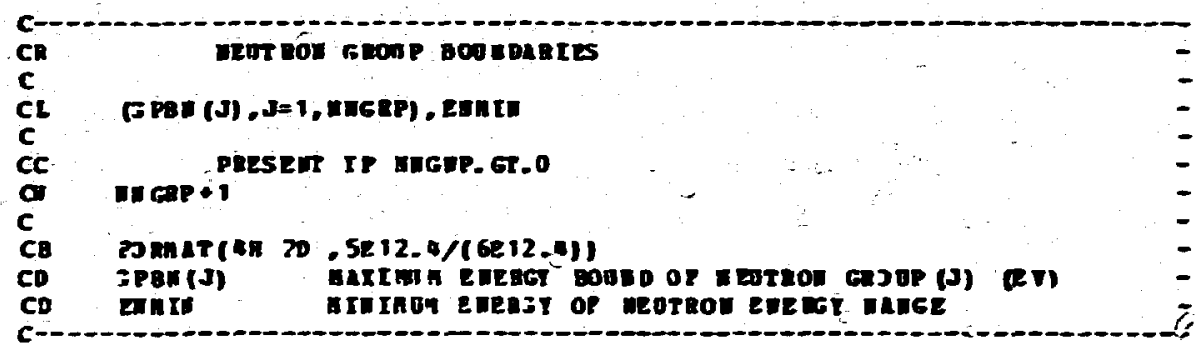

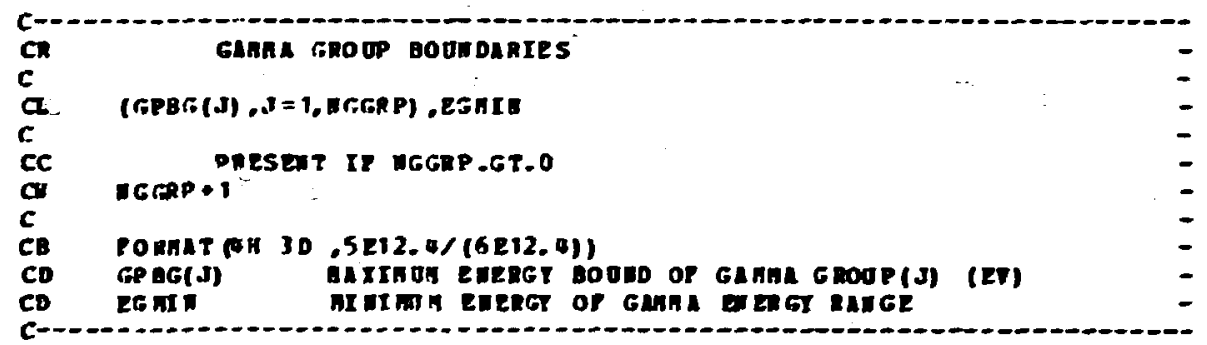

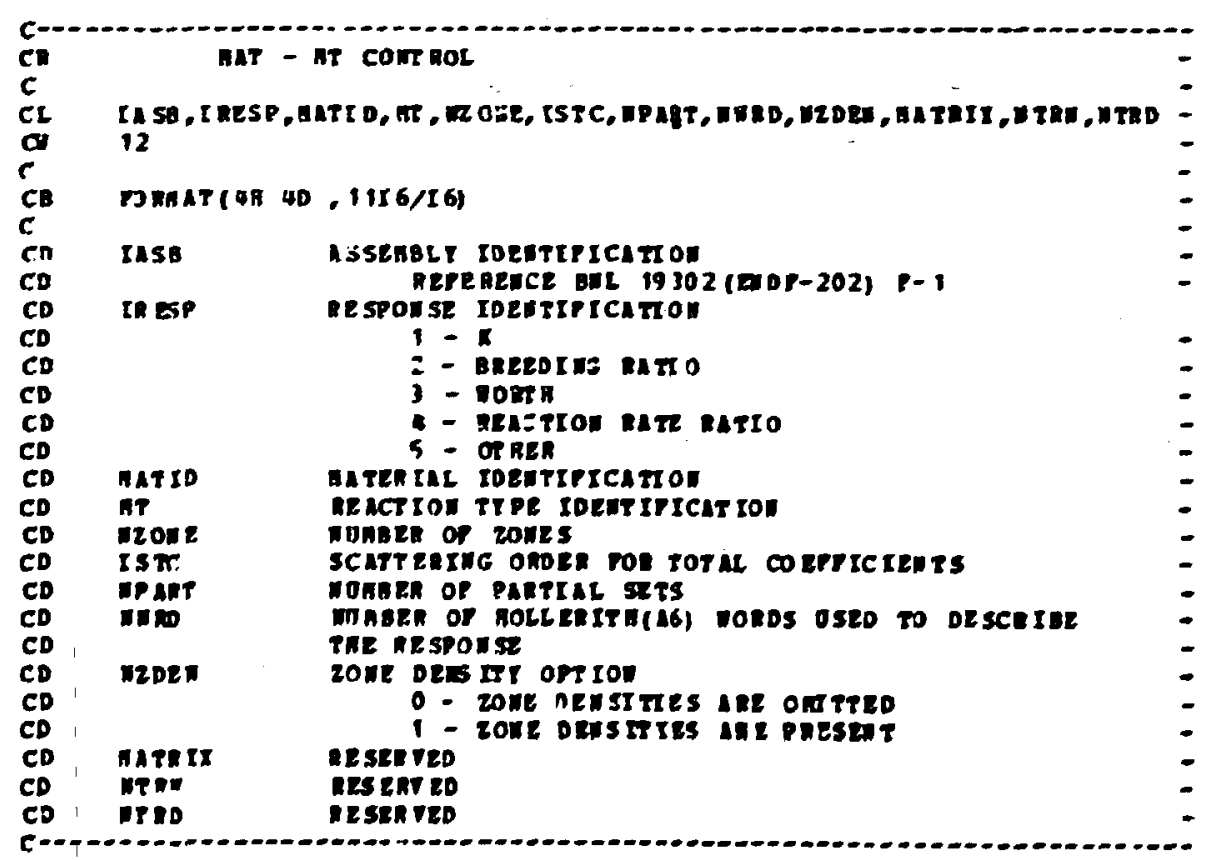




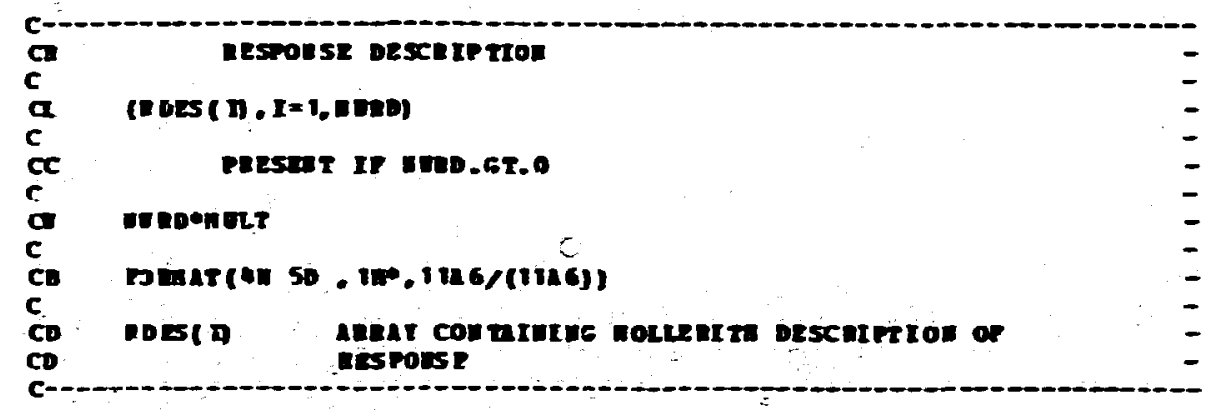

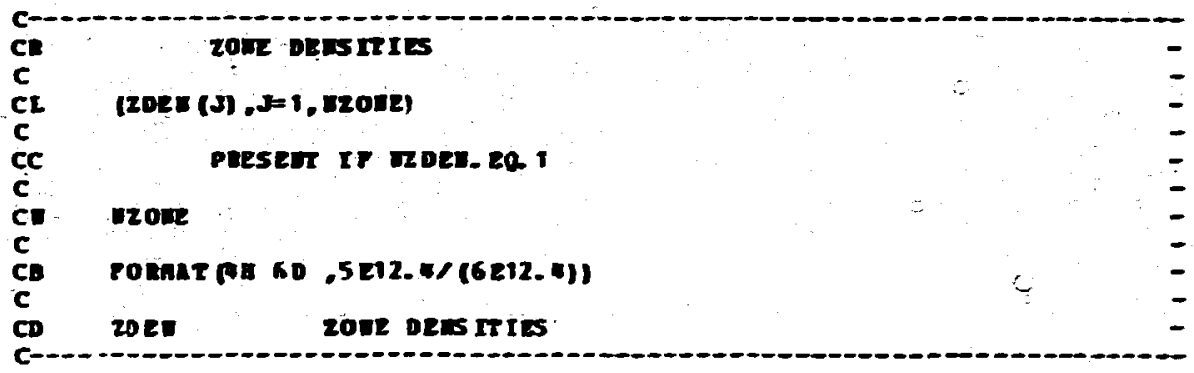

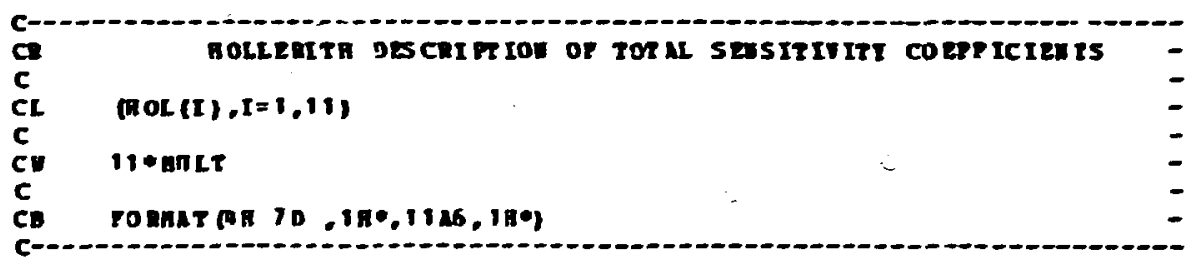

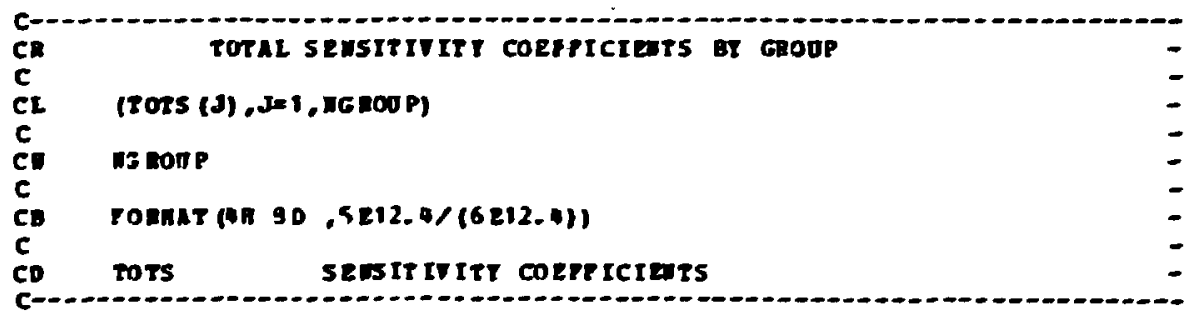


$[x-1]$
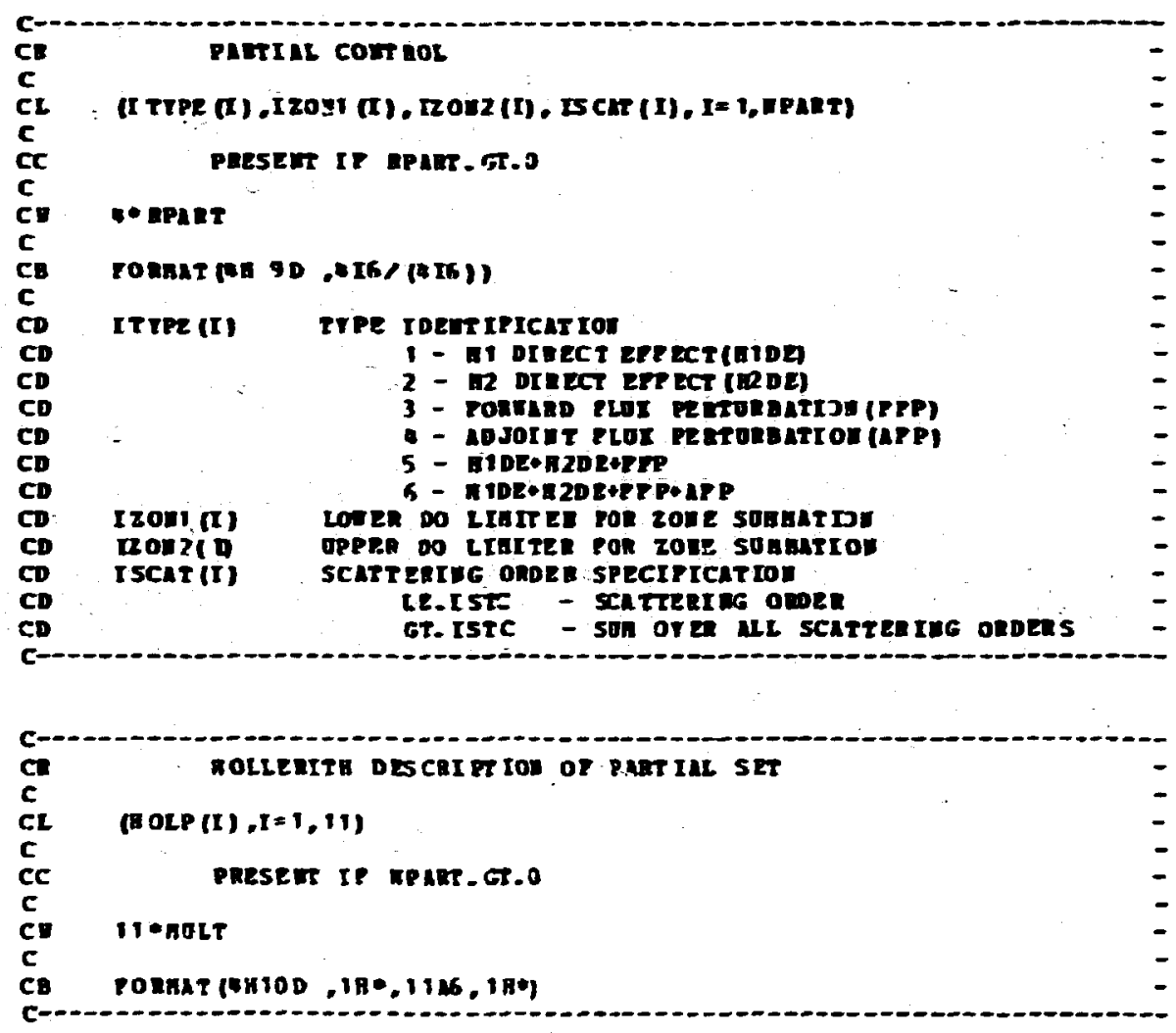

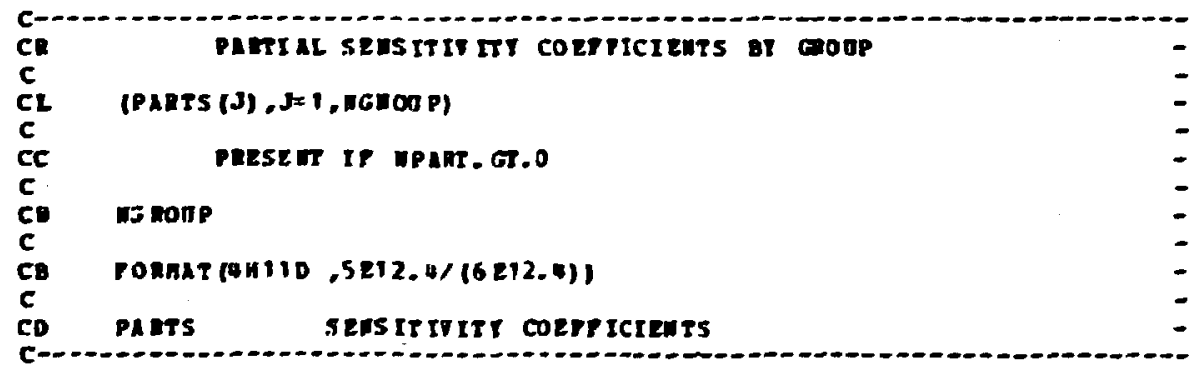


c

IX.18

Blank 
APPENDIX B

\section{The FIDO Input System}

The FIDO input method is especially devised to al low the entering or modifying of large data arrays with minimum effort. Special advantage is taken of patterns of repetition or symetry wherever possible. The FIDO system was patterned by Ward Engle and Wayne Rhoades after the input method used with the FLOCO coding system at Los Alamos, and was first applied to the DTF-II code. Since that time, numerous features requested by users have been added, a free-field option has been developed, and the application of FIDO has spread to innumerable codes.

The data are entered in units called "arrays." An array comprises a group of contiguous storage locations which are to be filled with data at one time. These arrays usually correspond on a one-to-one basis with FORTRAN arrays used in the program. p. group of one or more arrays read with a single call to the FIDO package forms a "block," and a special delimiter is required to signify the end of each block. Arrays within a block may be read in any order with respect to each other, but an array belonging to one block must not be shifted to another. The same array can be entered repeatedly within the same block. For example, an array could be filled with " 0 " using a special option, and then a few scattered locations could be changed by reading in a new set of data for that array. If no entries to the arrays in a block are required but the condition requiring the block is met, the delimiter alone satisfies the inn'-t requirement.

Three major types of input are available: fixed-field input, freefield input, and user-field input.

Fixed Field Input - Each card is divided into six 12-column data fields, each of which is divided into three subfields. The following sketch illustrates a typical data field. The three subfields always comprise 2,1 , and 9 columns, respectively.

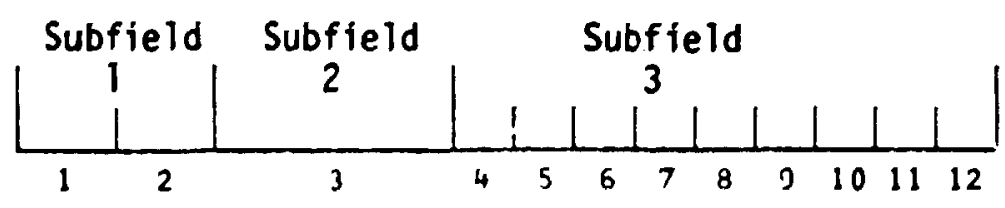


To begin the first array of a block, an array originator field is placed in any field on a card:

Subfield 1: An integer array identifier $<100$ specifying the data array to read

Subfield 2: An array-type indicator -

"\$" if the array is integer data

"*" if the array is real data

Subfield 3: Blank

Data are then place in successive fields until the required number of entries has been accounted for. A sample data sheet shown on page is illustrates this input.

In entering data, it is convenient to think of an "index" or "pointer" which is under control of the user, and which specifies the position in. the array into which the next data entry is to go. The pointer is always positioned at array location \#l by entering the array originator field. The pointer subsequently moves according to the data operator chosen. Blank fields are a special case, in that they do not cause any data modification and do not move the pointer.

A data field has the following form:

Subfield 1: The data nurrerator, an integer $<100$. We refer to this entry as $N_{1}$ in the following discussion.

Subfield 2: One of the special data operators listed below.

Subfield 3: A nine-character data entry, to be read in $\mathbf{F 9 . 0}$ format. It will be converted to an integer if the array is a " $\$$ " array or if a special array operator such as "Q" is being used. Note that an exponent is permissible but not required. Likewise, a decimal is permissible but not required. If no decimal is supplied it is assumed to be immediately to the left of the exponent, if any; and otherwise to the right of the last column. This entry is referred to as $N_{3}$ in the following discussion. 


\begin{tabular}{|c|c|c|c|}
\hline & & IOENTIFICATION & REMARKS (DO NOT PUNCH) \\
\hline 1,5 & 111 & & Begin the is array, fixed-field, integral \\
\hline 1 & $1,1,1,1,1,1$ & & Enter 1. \\
\hline$\perp, F$ & $1,1,1,1,12$ & & Fill array with 2. \\
\hline+2, & $1,1,1,1,1,1$ & & Begin the $2^{*}$ array, flxed-field, real. \\
\hline 1,1 & $1,1,2,3,4,1,1,1$ & $n$ & Enter 1.234. \\
\hline 1 & $1,2, \cdot, 3,4,2,-1$ & $1,1,0$ & $" 11$ \\
\hline,$s_{1}=$ & $1,2,3,4,1,1+0,2$ & & " " \\
\hline$\because "-3_{1}-$ & $1,1,1,12,3,4$ & & $" \quad "$ \\
\hline " & $1,1,1,1,1,7$ & & 17.0 \\
\hline$"$ & & & A blank field is always ignored. \\
\hline 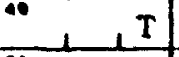 & 1 & $n$ & Terminate this block. \\
\hline$\because 1 ـ$ & $1,1,1 / 1,1 / 1$ & $1,1,1,2,0$ & No entries may follow $T$ on a card. \\
\hline$, 3, \cdots$ & & & Begin $3^{\text {th }}$ array, flxed-field roal. \\
\hline 1"9,I & $1,1,1,1,1,0$ & & Enter $0,1,2,3,4,5,6,7,8,9,10,10,10$. \\
\hline $3, R$ & $1,1,1,1,1,0$ & & as real numbers. \\
\hline $3, \cdots$ & $1,1,0, s, 1,0$, & & Repeat $3^{*}$ in free-1iold, skip \\
\hline 1,1, & $1,2,1,1,1,1$ & " & to 11th entry, correct sequence to \\
\hline 11 & $1,1,1,1,1,1$ & $1,1,1,3,0$ & $\ldots-9,10,11,12$ \\
\hline $4, \cdots$ & $\because I_{1} 1_{1}, 4_{1}, 0_{1}$ & & Begin 4k array, free-field, real. \\
\hline $2, Q, 4$ & $1 / 1 / 1 / 1 / 1 / 1 / 1$ & & Enter $1,2,3,4,1,2,3,4,1,2,3,4$ \\
\hline & $1, E_{1}$ & & End reading this array; remainder of array \\
\hline$" 1$ & $T_{1}{ }_{1}$ & & Terminate this block: \\
\hline 11 & $1,1,1,1,1$ & $\because$ & ". \\
\hline$\because 1$ & $1,1,1,1,1,1$ & $1,1,1,14,0$ & $\cdot$ \\
\hline
\end{tabular}


A list of data operators and their effect on the array being input c. follows:

"Blank" indicates a single entry of data. The data entry in the third subfield is entered in the location indicated by the pointer, and the pointer is advanced by one. However, an entirely blank field is ignored.

"t" or "-" indicates exponentiation. The data entry in the third field is entered and multiplied by $10^{ \pm N_{1}}$, where $N_{1}$ is the data numerator in the first subfield, given the sign indicated by the data operator itself. The pointer is advanced by one. In cases where an exponent is needed, this option allows the entering of more significant figures than the blank option.

"\&" has the same effect as "+".

" $R$ " indicates that the data entry is to be repeated $N_{1}$ times. The pointer is advanced by $N_{1}$.

"I" indicates linear interpolation. The data numerator, $N_{1}$, indicates the number of interpolated points to be supplied. The data entry in the third subfield is entered, followed by $N_{1}$ interpolated entries equally spaced between that value and the data entry found in the third subfield of the next non-blank field. The pointer is advanced by $N_{1}+1$. The field following an "I" field is then processed normally, according to its own data operator. The "I" entry is especially valuable for specifying a spatial mesh. In "\$" arrays, interpolated values will be rounded to the nearest integer.

" $L "$ indicates logarithmic interpolation. The effect is the same as that of "I" except that the resulting data are evenly separated in log-space. This is especially convenient for specifying an energy mesh.

"Q" is used to repeat sequences of numbers. The length of the sequence is given by the trird subfield, $N_{3}$. The sequence of $N_{3}$ 
entries is to be repeated $N_{1}$ times. The pointer is advanced by $N_{1} * N_{3}$. If either $N_{1}$ or $N_{3}$ is 0 , then a sequence of $N_{1}+N_{3}$ is repeated one time only, and the pointer is advanced by $N_{1}+N_{3}$. This feature is especially valuable for geometry sfecification.

The "N" option has the same effect as "Q", except that the order of the sequence is reversed each time it is entered. This is valuable for the type of symmetry possessed by quadrature coefficients.

"M" has the same effect as " $N$ " except that the sign of each entry in the sequence is reversed each time the sequence is entered. For example, the entries:

$1232 M 2$

would be equivalent to:

$\begin{array}{lllllll}1 & 2 & 3 & -3 & -2 & 2 & 3\end{array}$

This option is aiso useful in entering quadrature coefficients.

" $Z$ " causes $N_{1}+N_{3}$ locations to be set to 0 . The pointer is advanced by $N_{2}+N_{3}$.

" $C$ " causes the position of the last arricy item entered to be printed. This is the position of the pointer, less 1. The pointer is not moved.

" 0 " causes the print trigger to be changed. The trigger is originally off. Successive " 0 " fields turn it on and of $f$ alternately. When the trigger is on, each card image is listed as it is read.

"S" indicates that the pointer is to skip $N_{1}$ positions leaving those array positions unchanged. If the third subfield is nonblank, that data entry is entered following the skip, and the pointer is advancea by $N_{1}+1$.

"A" moves the pointer to the position $N_{3}$, specified in the third subfield. 
" $F$ " fills the remainder of the array with the datum entered in the third subfield.

" $E$ ". skips over the remainder of the array. The array length criterion is always satisfied by an "E", no matter how many entries have been specifi.d. No more entries to an array may be given following an " $E$ ", except that data entry may be restarted with an "A".

The reading of data to an array is terminated when a new array origin field is supplied, or when the block is terminated. If an incorrect number of positions has been filled, an error edit is given, and a flag is set which will later abort extcution of the problem. FID0 then continues with the next array if an array origin was read. Otherwise, it returns control to the calling program.

A block termination consists of a field having " $T$ " in the second sub-field. All entries following " $T$ " on a card are ignored, and control is returned from FIDO to the calling program.

Comment cards can be entered within a block by piacing an apostrophe (') in column 1. Then columns 2-80 will be listed, with column 2 being used for printer carriage control. Such cards have no effect on the data array or pointer.

Free-Field Input - With free-field input, data are written without fixer restrictions as to field and subfield size and positioning on the card. The options used with fixed-field input are available, although some are slightly restricted in form. In gerieral, fewer data cards are required for a problem, the interpreting print is easier to read, a card listing is more intelligible, the cards are easier to keypunch, and certain common keypunch errors are tolerated without affecting the problem. Data arrays using fixed- and free-field input can be intermingled at wiil within a given biock.

The concept of three subfields per field is still applicable to freefield input, but if no entry for a field is required, no-space for it need be left. Only columns 1-72 may be used, as with fixed-field input. 
The array originator field can begin in any position. The array identifiers and type indicators are used as in fixed-field input. The type indicator is entered twice, to designate free-field input ( $:$.e:, "\$\$" or "**"). The blank third subfield required in fixed-field input is not required. For example: $31^{\star \star}$ indicates that array 31 , a realdata array, will follow in free-field format.

Data ficlds may follow the array origin field immediately. The data field entries are identical to the fixed-field entries with the following restrictions:

(1) Any number of blanks may separate fie!ds, but at least one blank must follow a third subfield entry if one is used.

(2) If both first and second subfield entries are used, no blanks may separate them, i.e., 245 , but not $24 \mathrm{~S}$.

(3) Numbers written with exponents must not have imbedded blanks, i.e., $1.0[+4,1.0 E 4,1.0+4$, or even $1+4$, but not $1.0 \mathrm{E} 4$.

(4) In third-subfield data entries, only 9 digits, including the decimal but not including the exponent field, can be used, i.e., 123455.89E07, but not 123456.789E07.

(5) The $Z$ entry must be of the form: $738 Z$, not 2738 or $738 Z$.

(6) The + or - data operators are not needed and are not available.

(7) The $Q, N$, and $M$ entries are restricted: $3 Q 4$, IN4, or M4, but not $4 Q, 4 N$, or $4 M$.

User-Field Input - If the user follows the array identifier in the array originator field with the iharacter " $U$ " or " $V$ ", the input format is to be specified by the user. If " $U$ " is specified, the FORTRAN FORMAT to be used must be supplied in columns 1-72 of the next card. The format must be enclosed by the usuai parentheses. Then tie data for the entire array must follow on successive cards. The rules of ordinary FORTRAN input as to exponents, blanks, etc., apply. If the array data do not fill the last card, th? remainder must be left blank.

" $V$ " has the same effect as " $U$ " except that the format read in the last preceding "U" array is used. 


\section{7) 3.

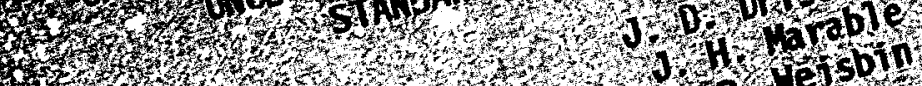

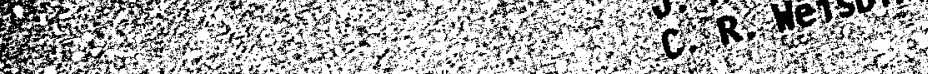 \\ C.R R t te

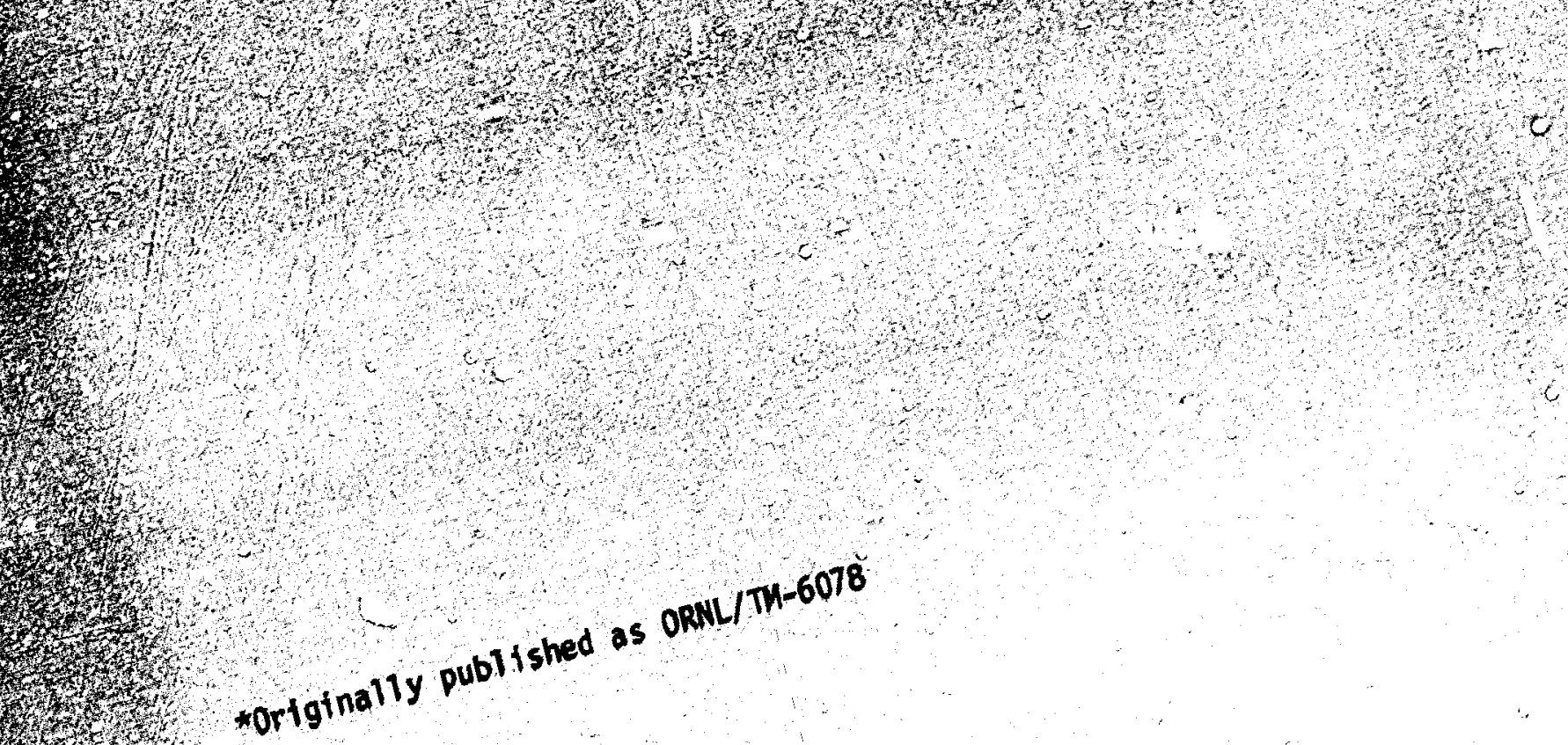

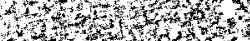
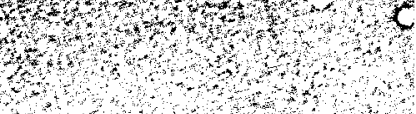


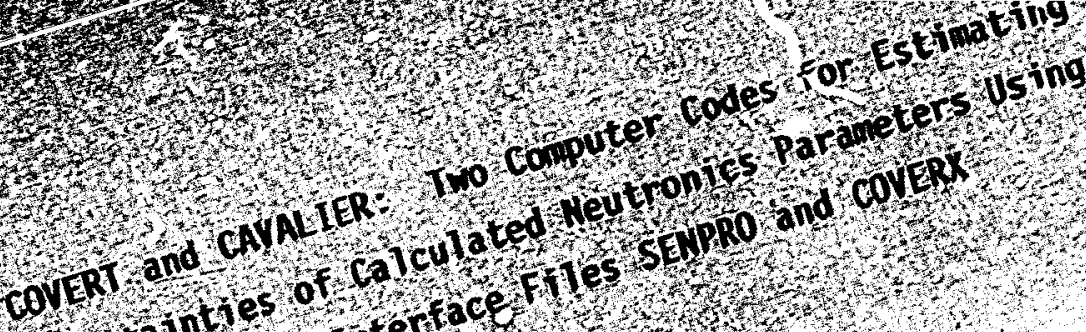

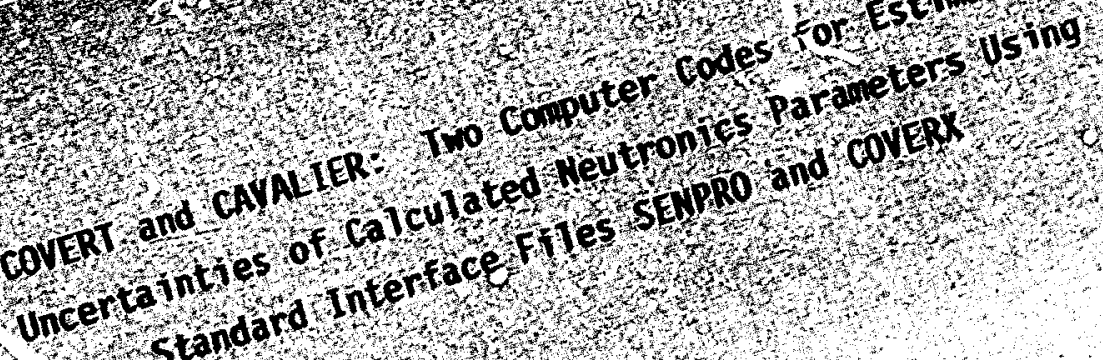

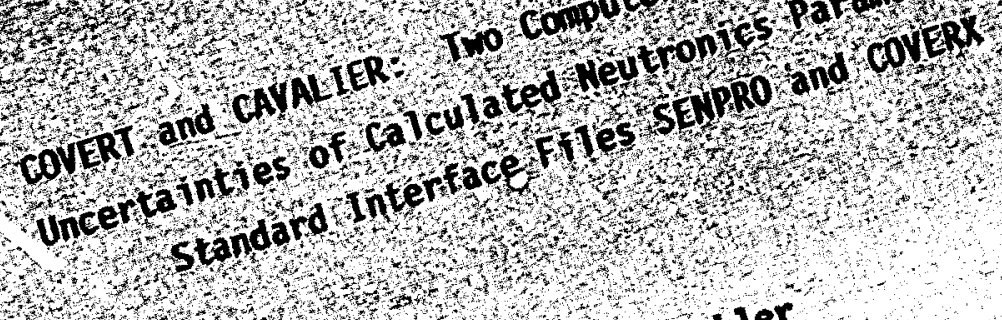

J. D. Drischler 3. H. parable Date Published - August 7978

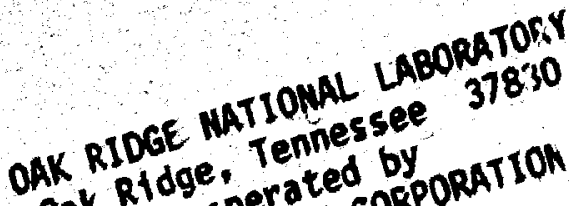
Oak RT operated by UNION CARBIDE CORPORATION DEPARTMENT OF ENERGY 


\section{Page}

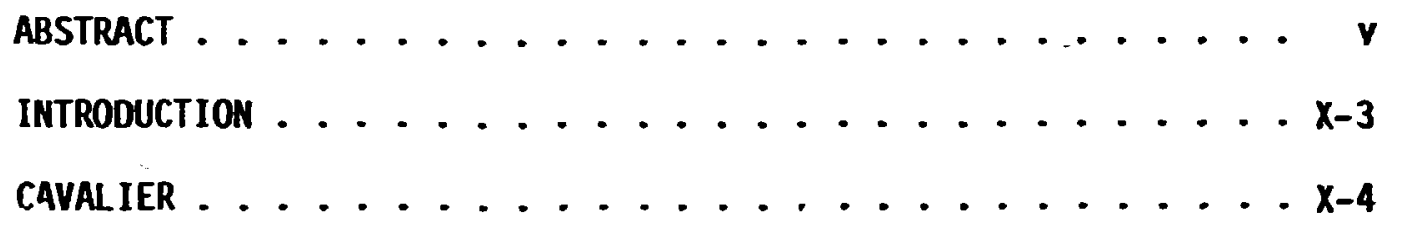

Sample Problem for CAVALIER . . . . . . . . . X-6

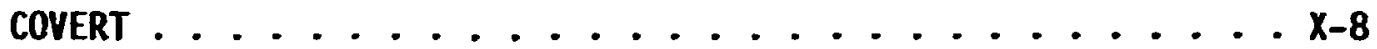

Sample Problen for COVEPT . . . . . . . . . . . X-9 REFERENCES ...................... APPENDIX A. The Format for Standard Interface File COVERX for Multigroup Cross-Section Covariance Matrices . . X-11

APPENDIX B. The FIDO Input System .............. X-17 


\section{ABSTRACT}

A description is given of the computer code CAVALIER, which provides a simpie means for estimating the uncertainties in calculated neutronics parameters that result from uncertainties in nuclear data. This code uses predetermined detailed multigroup sensitivity coefficients in SENPRO format for specific reactor and/or shielding assemblies and multigroup cros=-section covariance uncertainties in COVERX format. Also described is the computer code COVERT, which converts unformatted (binary) covariance files to card image form and vice versa. This is useful for transferring covariance files from one installation to another. These two codes, along with extended compilations of sensitivity ccefficients and covariance matrix libraries are currently available through the Radiation Shielding Information Center (RSIC) at Oak Ridge National Laboratory. 


\section{INTRODUCTION}

ThE ORNL sensitivity and uncertainty analysis system (FORSS) ${ }^{1}$ has been applied to several data-testing CSEMG benchmarks ${ }^{2}$ in order to determine, for calculated performance parameters, the uncertainties which result from uncertainties in nuclear data. Interface files for multigroup sensitivity coefficients in SENPRO format ${ }^{3}$ are used herein, as well as multigroup cross-section covariance interface files which use the COVERX format. ${ }^{4}$ These forrats svere developed according to the standards established by the Committee on Computer Codes Coordination (CCCC). ${ }^{5}$ The description of the COVERX format is given in Appendix A.

In order to use the covariance information from computerized data $\mathrm{fi}^{\prime} \quad$ it is necessary to have codes which can read such files as well a to solve the pertinent algorithms. This report documents two su. odes. The first, CAlALIER, is used to estimate the uncertainties in calcuiated neutronics parameters using sensitivity coefficients ${ }^{6}$ in SENPRO format and covariance data in COVERX format. The second, COVERT, is useful for converting a covariance file from binary to card image (and vice versa), and for obtaining a printed edit of the nuclear data covariance file. This code is essential since COVERX unformatted (binary) data, for computational efficiency, are not easily read on different types of computers. To facilitate the transfer of data from one installation to another, COVERT can translate a binary file to card image form and vice versa. 
CAVALIER

The program CAVALIER computes the relative standard feviat ions of calculated performance parameters of reactor systens. These standard deviations are assumed to result from specific nuclear data errors, the covariances of which are contained in a COYERX file. These nuclear data covariances are used with sensitivities in a SENPRO file to determine the standard deviation for response $R$ by the following equation:

$$
\left[(S . D .)^{R}\right]^{2}=\sum_{\substack{i, j \\ g g^{\prime}}} s_{g}^{R}(\operatorname{cov}){ }_{g^{\prime}, g^{\prime}}^{i, j} s_{g^{\prime} j}^{R}
$$

where: $\quad S_{g i}^{R}$ is the relative sensitivity coefficient for response $R$ to the cross section $\sigma_{g}{ }^{i}$ of type $i$ (denoting nuclide and reaction) in group $g$. This sensitivity is given by

$$
s_{g i}^{R}=\frac{\sigma_{g}^{i}}{R} \frac{\partial R}{\partial \sigma_{g}^{i}}
$$

$(\operatorname{cov})_{g}^{i, j}$, is the relative covariance of crass sections $\sigma_{g}^{i}$ and $\sigma_{g^{\prime}}{ }^{j}$.

$(\text { S.D. })^{R}$ is the standard deviation of response $R$ resulting

from the uncertainties included in the sum.

The input data corisist of:

(1) Problem type specifications for labeling purposes.

(2) The number of parameter sets $N$. A set consists of nine identifiers describing a sensitivity profile and covariance matrix to be included in the calculation. Provisions are made ior reporting resists from searches on one or more of the above parameters as described below.

(3) The printer output data-set reference number (default value 6), the SENPRO sensitivity file data-set reference number (default value 10 ), and the COVERX covariance file jata-set reference number (default value II). 
(1) The binary sensitivity file in SENPRO format.

(5) The binary covariance matrix file in COVERX format.

The output consists of:

(1) The input information.

(2) Problem type identification.

(3) Materials and reactio is of interest which are selected from the cuvariance matrix file with associated wamings if a covariance is not found in the file.

(4) Assemblies and responses of interest wich are selected from the sensitivity file with associated warnings if a sensitivity is not found in the file.

(5) The correlations included in the problem.

(6) The resulting relative standard deviation $\left(\begin{array}{l}\% \\ 0\end{array}\right)$ for eack of the calculated performance parameters of interest.

(7) The coritributions from covariances in input cross section data to the output relative variance of the indicated response.

Specifically, the input to CAVALIER for one case consists of one card of 5 numbers and one array entered in FIDO system format. The FIDO system is described in ppendix B. Several stacked cases can be calculated in one run. The following input is required for each case:

one card (format 515): $\quad$ NB, N, LUNIT, MNIT, IOUT sensitivity \& covariance matrix identifiers: $5 \$ \$((I N F O(I, J), I=1,9), J=1, M)$ FIDO terminator:

$N B=1,2,3$ according to problem type selection, fast reactor benchmark, shielding benchmark, or thermal reactor benchmark.

$N=$ the number of parameter sets identifying the sensitivity profiles and the covariance matrices to be included.

LUNIT = the data-set reference number of the sensitivity file in SENPRO format (default value $=10$ ).

MUNIT = the data set reference number of the covariance file in COVERX format (default value $=11$ ). IOUT $=$ printer output data-set refarence number (default value $=6$ ). 
INFO $(I, J) I=1,9 J=1, N$, consists of nine identifiers for the sensitivity profile $J$. The nine identifiers in order are: IASB = the assembly identifier as it appears in the file or, if the first IASB $(=\operatorname{INFO}(1,1))$ is negative, searches are pade to include every assembly in the file. (Assembly identifiers. were selected as repor'eo in Ref. 2. Arbitrary numbers were chosen by the file cieator in cases where assembly identification dic not exist.)

IRESP $=1,2,3,4,5$ according as the response is multiplication factor $k$, breeding ratio, worth, reaction rate ratio, or some other type of response. If the first $\operatorname{IRESP}(=\operatorname{INFO}(2,1)$ ) is negative, all responses are included by the search. MATID = nuclide identification using ENDF assignments. $M T=$ reaction identification using ENDF assignments STRN $=1,2,3, \ldots$ an identification number chosen by the file creator (presently arbitrary) to distinguish different reaction rate or worth responses ( 0 for IRESP $=1$ ).

ITYPE, IZON1, IZON2, ISCAT refer to partial sensitivities and are set to zero since no partial sensitivities are presently used.

Sample Problem for CAVALIER:

This problem determines the relative standard deviations in percent of the calculated response(s) due to estimated uncertainties in $238 u(n, f)$, ${ }^{238} \mathrm{u}(\mathrm{n}, \gamma), 239 \mathrm{Pu}(\mathrm{n}, \mathrm{f}),{ }^{239} \mathrm{Pu}(\mathrm{n}, r), 23=\mathrm{Pu}(\bar{v})$ including the correlation setween $239 p_{u}(n, f)$ and the ${ }^{239} \mathrm{pu}(n, r)$ cross sections. The calculated responses for this sample are multiplicatian factor (IRESP $=1$ and NTRN=0), central reaction rate rat:os ${ }^{28} \mathrm{c} / 49 \mathrm{f}$ (IRESP $=1$ and $N T R N=1$ ), and $28 \mathrm{f} / 49 \mathrm{f}$ (IRESF $=4$ and $N T R N=2$ ) of the ZPR-6/7 assembly. (IASB=5 is the number assigned this assembly in the SENPRO file reported in Ref. 2.) The relative standard deviation for the three responses using CAVALIER are:

\begin{tabular}{lc:c} 
Assembly & Response & Standard Deviation $(\%)$ \\
\hline $2 P R-5 / 7$ & $k$ & 3.57 \\
$Z P R-6 / 7$ & ${ }^{28} \mathrm{c} / 49 \mathrm{f}$ & 8.86 \\
$2 P R-6 / 7$ & $28 \mathrm{f} / 49 \mathrm{f}$ & 4.85
\end{tabular}


This sample problem uses the fast benchmarks for which sensitivities are rap: $: \pm=1$ in Ref. 3 and the covariance matrices from the LAFBR Core Physics Cofariance Fatrix Library. ${ }^{4}$ He assume these sensitivities and covariances are availabie in binary SENPRO and COVERX formats and reside on units having data set reference numbers 10 and 11.

The input, as will be show below, can be stacked as three cases. The first searcies through all the responses in the SENPRO file (since INFO $(2,1)$ is negative) and calculates a standard deviztion for each response found. In this example the ZPR- $/ 7$ assembly was specifically selected and five MAT-MT pairs were specifically identified.

The second of the three input cases fin. the standard deviations for all assemblies (INFO $(1,1)$ is negative) and all responses (INFO $(2,1)$ is negative) in the file, but five nuclide and reaction types (MAT-MT pairs) were specifically identified.

The third input case calculates for 311 assemblii.s and responses in the SENPRO file, the standard deviations which result from errors in all nuclide reaction types residing in the COVERX file ( $N$ is negative).

The input data files are as follows:

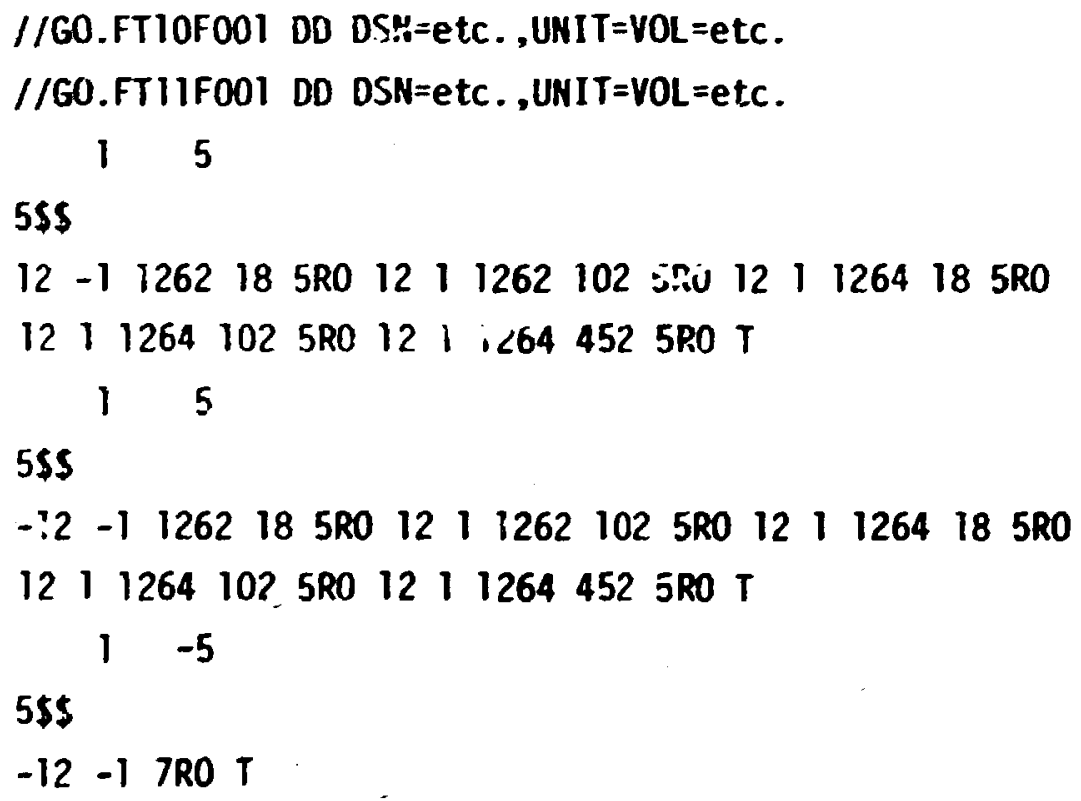

The output prints a warning message to note whenever a sensitivity or covariance is missing from the file, and these sensitivities and covariances arr set to zero. Correlations, such as those between the ${ }^{239} \mathrm{pu}(\mathrm{n}, \mathrm{f})$ and the ${ }^{2} \mathrm{Pu}(n, y)$ cross sections in the first example are automatically selected. included in the calculation, and flagged in the printer output. 


\section{COVERT}

The program COVERT copies a covariance file in COVERX format, and in the copying process changes the file representation from binary to card image form or vice versa. In addition, a complete listing or a partial listing of the file is printed to give the user information about the contents of a particular file.

There are tró (2) input files:

(1) The COVERX covariance file to be converted which is specified by the FORTRAN data-set reference number NIN (default value 20).

(2) The control file wich corsists of a single card (card image) wich is specified by FORTRAN data-set reference number 5. This card consists of four integers in (415) format: NBE, NIN, NOUT, H06.

$$
\begin{aligned}
& \text { MBE }=0 \text { conversion is binary to card image. } \\
& \neq 0 \text { conversion is card image to binary. } \\
& \text { NIN = FORTRAN data-set reference number of the input }
\end{aligned}
$$

There are two output files (both referred to above):

(1) The converted covariance file with data-set reference number NouT (default value 21 ).

(2) The file of printer output with data-set reference number specified by NOG (default value 6 ). 
Sample Problem for COVERT:

The input on unit 5 consists of a single blank card. Unit 20 contains the binary COVERX file. On unit 21 is a dumb file.

$/ / 60 . F T 20 F 001$ DD UNIT $=3330, \mathrm{VOL}=S E R=2 \times 0000,0 I S P=S H R$, // DSH=JDD. COVERX.FISS.SPEC. VI . OCT2477

!/GO.FT2IFOOI DD DUMY

//60.FT05FO01 DD *

(? blank card)

/*

The blank card could be replaced by the following:

$$
\begin{array}{llll}
0 & 20 & 21 & -6
\end{array}
$$

with the same result.

The printer output on unit 6 produces ? reduced edit of the COVERX file on unit 20 . 


\section{REFERENCES}

1. C. R. Heisbin, J. H. Marable, J. L. Lucius, E. M. Oblow, F. R. Mynatt, R. W. Peelle, and F. G. Perey, "Application of FORSS Sensitivity and Uncertainty Methodology to Fast Reactor Benchmark Analysis," ORNL/TM-5563 (1976).

2. "Cross Section Evaisation Working Grocp Benchmark Specifications," BPL-19302 (ENDF-202) (November 1974).

3. J. H. Marable, J. L. Lucius, and C. R. Meisbin, "Compiistion of Sensitivity Profiles for Several CSEMG Fast Reactor Benchmarks," Oak Ridge National Laboratory report ORNL-5262 (ENDF-234) (March 1977).

4. J. D. Orischler and C. R. Heisbin, "Compilation of Multigroup CrossSection Covariance Matrices for Several Important Reactor Materials." ORNL-5318 (ENDF-235) (October 1977).

5. B. M. Carmichael, "Standard Interface Files and Procedures for Reactor Physics Codes, Version III," Los Álamos Scientific Laboratory report LA-5486-MS (February 1974).

6. J. H. Marable, J. D. Orischler, and C. R. Keisbin, "SENDIN ard SENTINEL: Two Computer Codes to Assess the Effects of Nuclear Data Changes," ORNL/TM-5946 (ENDF-250) (July 1977). 


$$
x-1 ;
$$

APPEMDIX A

The Formai for Stzndard Interface File COVERX for Multigroup Cross-Sertion Covariance Matrices 


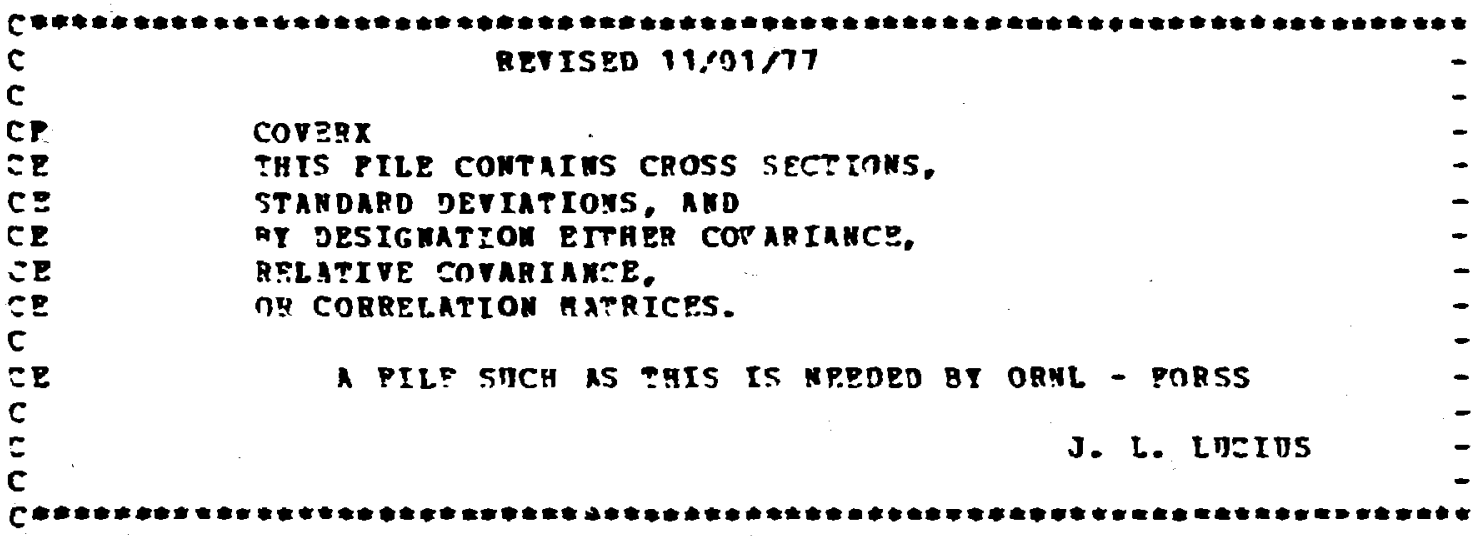

\begin{tabular}{|c|c|c|c|}
\hline $\begin{array}{cl}\mathrm{cs} \\
\mathrm{cs}\end{array}$ & PILE STRUCTHRE & & - \\
\hline $\begin{array}{l}\text { cs } \\
\text { es }\end{array}$ & EECORN TYPE & PRES PI: IP & - \\
\hline Es & 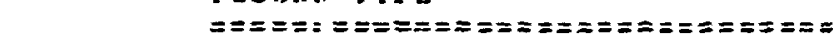 & 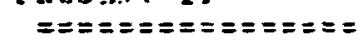 & - \\
\hline cs & PILE IDENTIPICATION & ALEATS & - \\
\hline cs & =ILE CCRTROL & ALEATS & - \\
\hline Cs & FILP DESCPIPT ION & NHOLL.GT.O & - \\
\hline CS & NEOTRNN GROUP BOTKDAPIES & WNGRIP. GT.J & - \\
\hline Cs & GAMAA GROUP BOURDARIES & 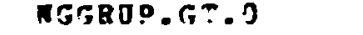 & - \\
\hline CS & HAT - HE CONTROL & ALUATS & - \\
\hline Cs & $* * * *+* * *+* * * *$ (RPQPEAT POQ ALL HATESIAL- & - & - \\
\hline es & REACTION TYPE PAIXSI & & - \\
\hline Cs & MAT- $9 T$ CSOSS SECTION AVD & ALWAIS & - \\
\hline c5 & - STANDAPD DEVIATIONS & & - \\
\hline CS & $+*+*+\infty+* *+\infty *+\infty$ & & - \\
\hline $\operatorname{cs}$ & & & - \\
\hline$\overline{c s}$ & 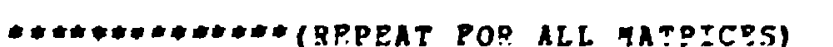 & & - \\
\hline Cs & - MATAIX CONTROL & ALEATS & - \\
\hline Cs & - AlOCR CONTBOL & ALWAYS & - \\
\hline Cs & $* * * * * * * * *(R E P E A T$ POR ALL BLOCKS) & & - \\
\hline es & MRTRIX TATA & ALWAYS & - \\
\hline
\end{tabular}

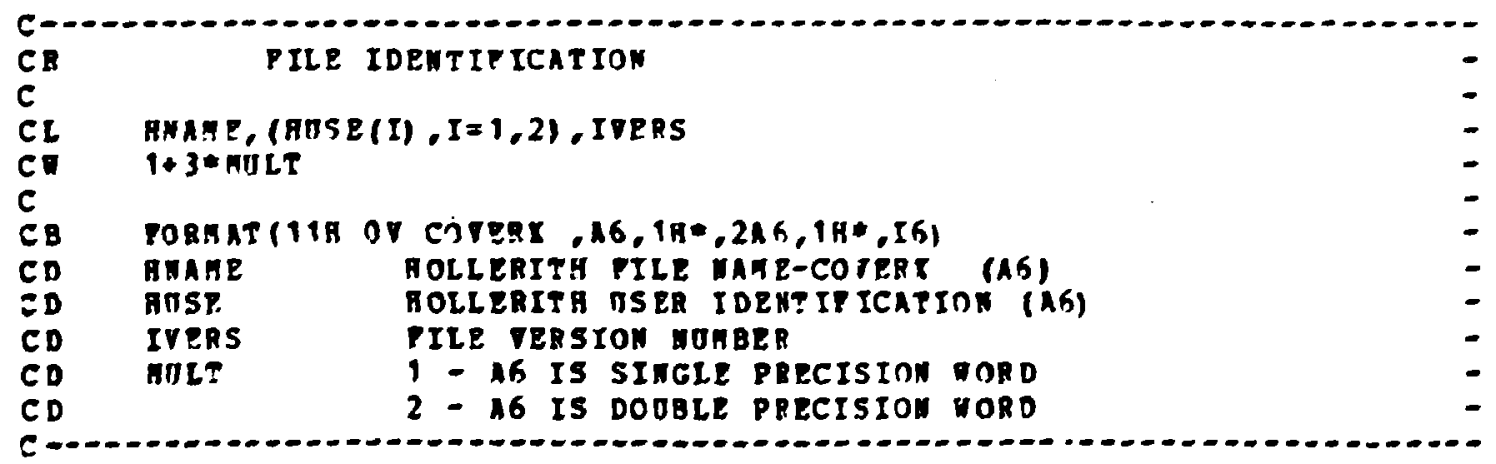




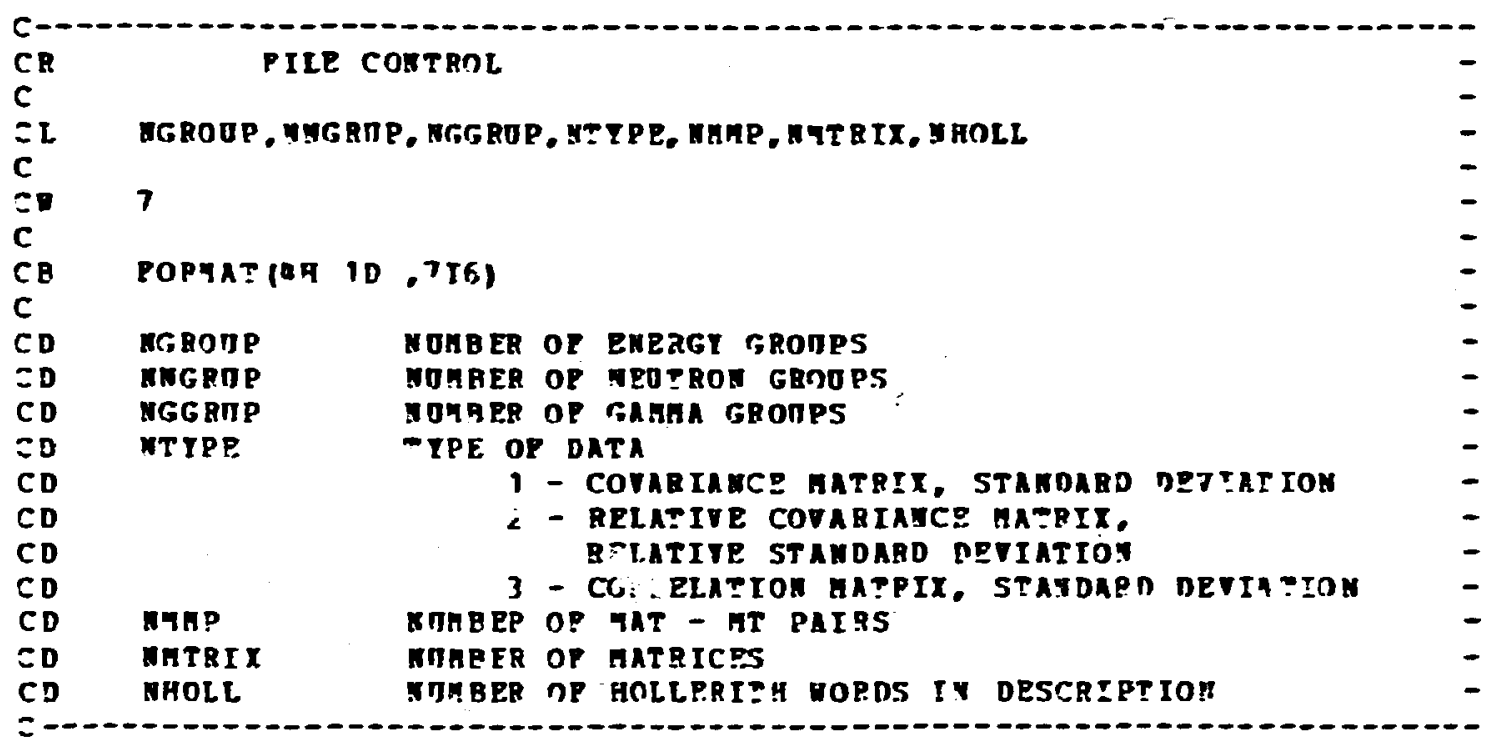

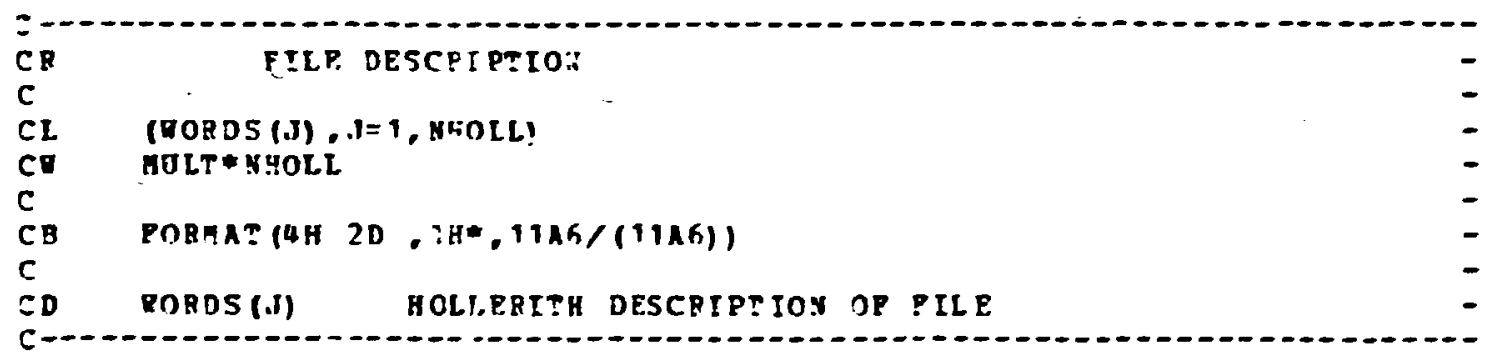

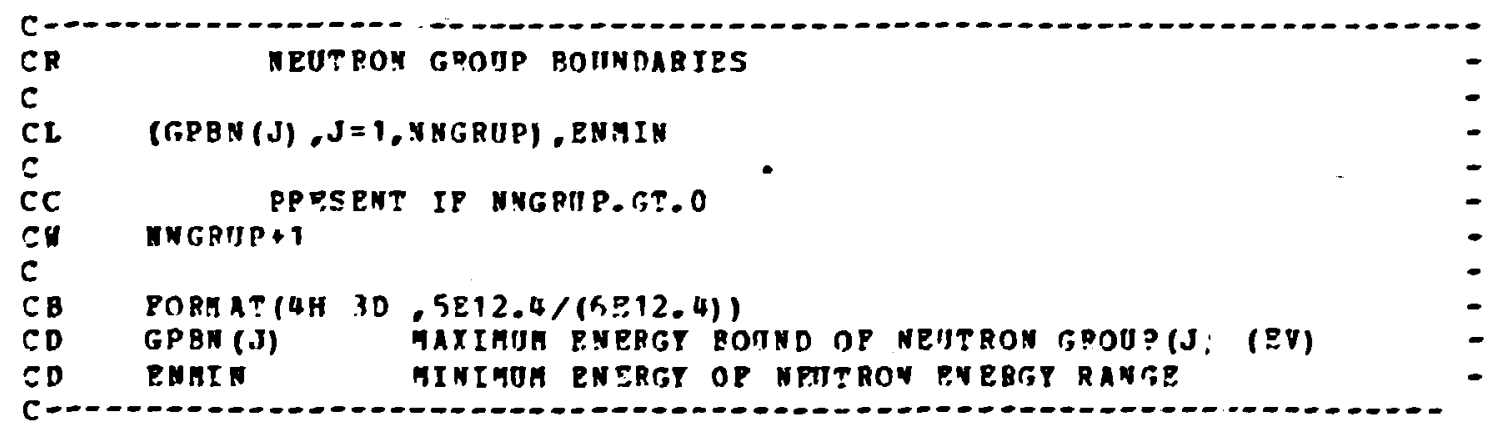

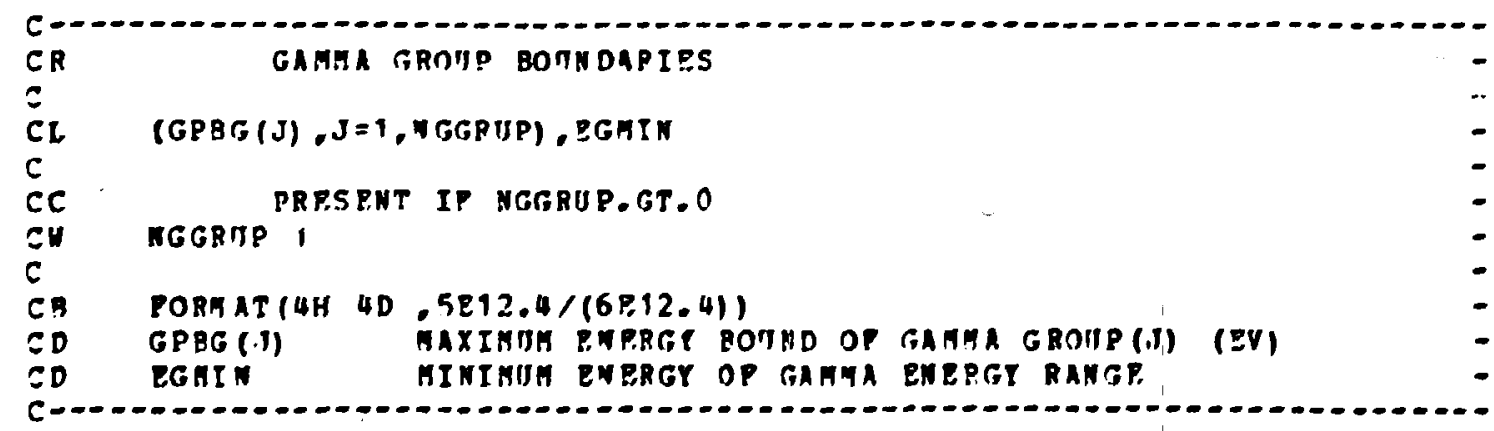




\section{$x-14$}
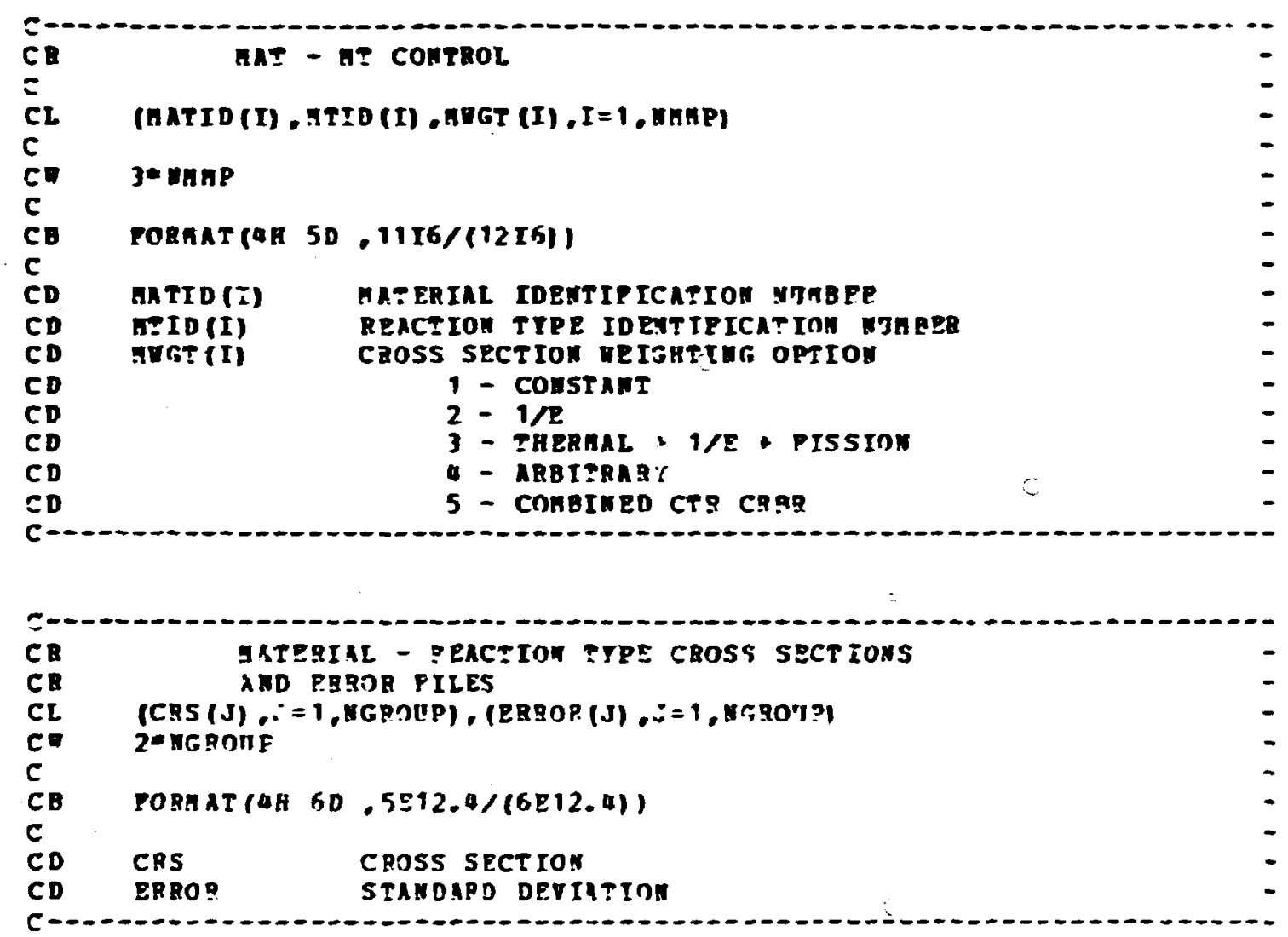

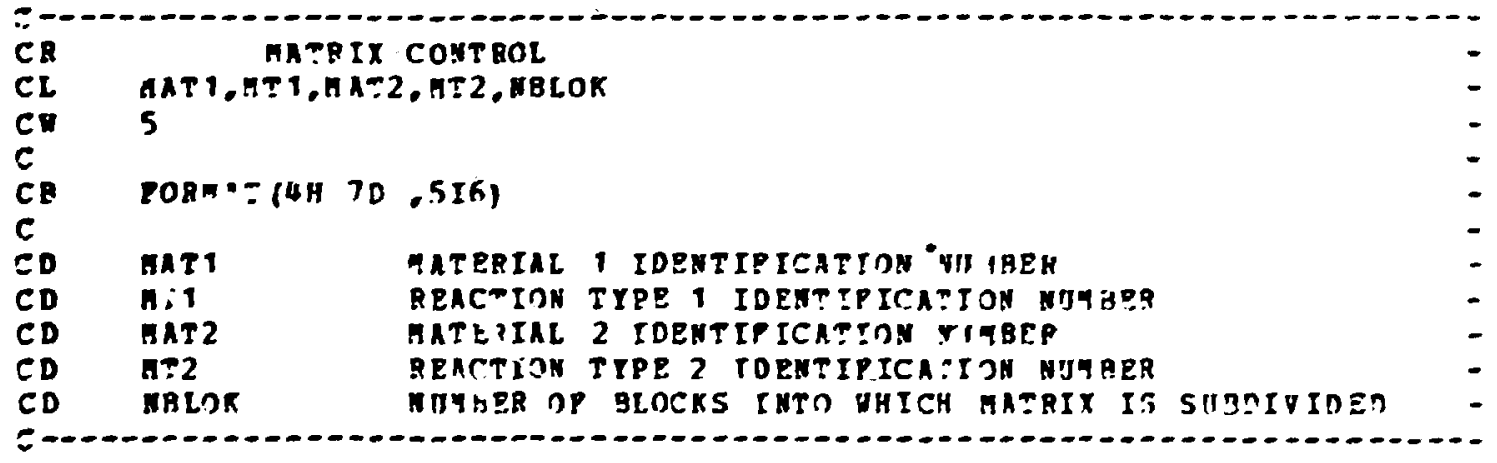

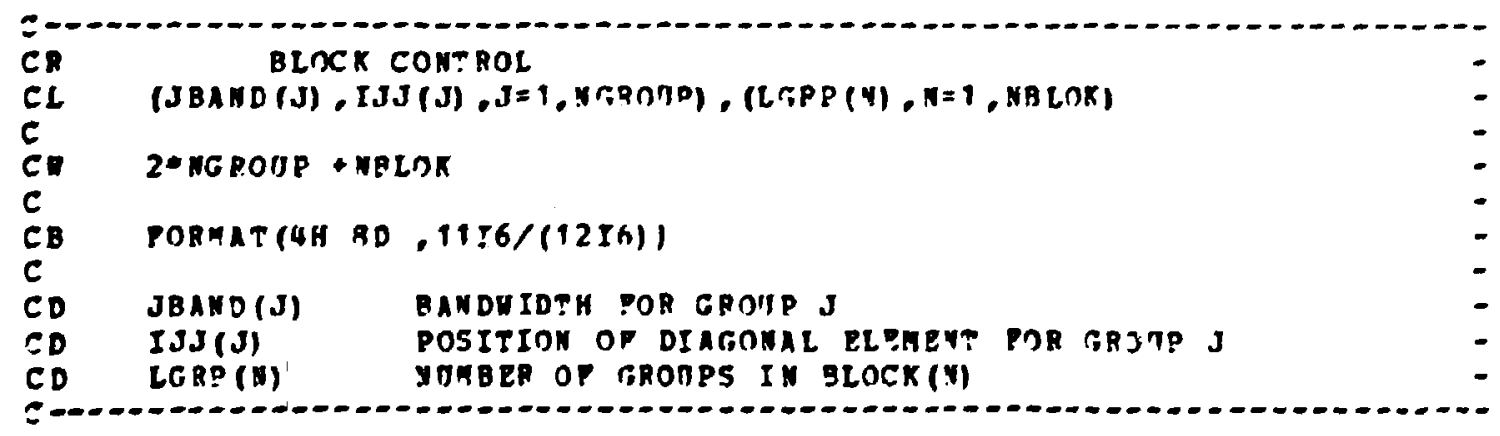




\section{$x-15$}

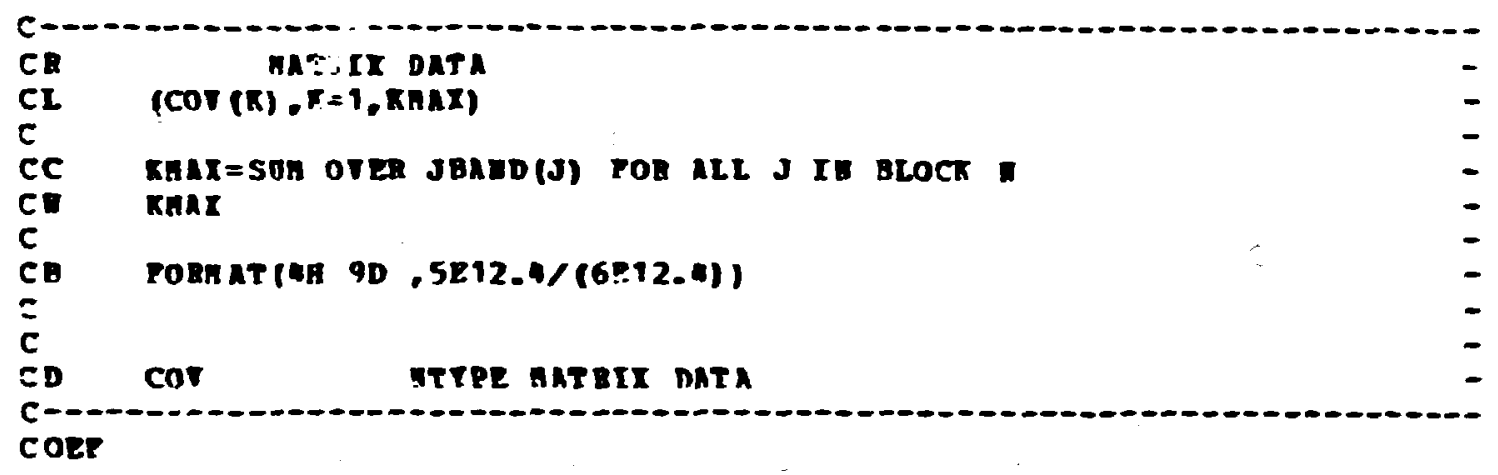


APPENDIX B

The FIDO Input System

The FIDO input methed is especially devised to allow ti-e entering or modifying of large data arrays with minimum effort. Special advantage is taken of patterns of repetition or symmetry wherever possible. The FIDO system was patterned by Ward Engle and Wayne Rhoades after the input method used with the FLOCO coding system at LOS Alamos, and was first applied to the DTF-II code. Since that time, numerous features requested by users have been added, a free-field option has been developed, and the application of FIDO has spread to innumerable codes.

The data are entered in uni.s called "arrays." An array comprises a group of contiguous storage locations which are to be filled with data at one time. These arrays usually correspond on a one-to-one basis with FORTRAN arrays used in the progran. A group of one or more arrays read with a single call to the FIDO package forms a "block," and a special delimiter is required to signify the end of each block. Arrays within a block may he read in any order with respect to each otiner, but an array belonging to one block must not be shifted to another. The same array can be entered repeatedly within the same block. For example, an array could be filled with " 0 " using a special option, and then a few scattered locations could be changed by reading in a new set of data for that array. If no entries to the arrays in a block are required but the condition requiring the block is met, the delimiter alone satisfies the input requirement.

Three major types of input are available: fixed-field input, freefield input, and user-field input.

Fixed Field Input - Each card is divided into six 12-column data fields, each of wich is divided into three subfields. The following sketch illustrates a typical data field. The three subfields always comprise 2, 1 , and 9 columns, respectively.

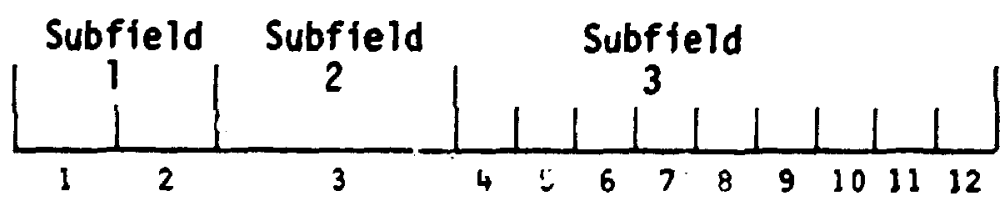


To hegin the first array of a block, an array originator field is placed in any field on a card:

Subfield 1: An integer array identifier < 100 specifying the data array to read

Subfield 2: An array-type indicator -

"f" if the array is integer data

"* if the array is real data

Subfield 3: Blank

Data are then place in successive fields unti? the required number of entries has been accounted for. A sample data sheet shom on page 19 il)ustrates this input.

In entering data, it is convenient to think of an "index" or "pointer" which is under control of the user, and wich specifies the position in the array into wich the next data entry is to go. The pointer is always positioned at array location 1 by entering the array originator field. The pointer subsequently moves according to the data operator chosen. Blank fields are a special case, in that they do not cause any data modification and do not move the pointer.

A data field has the following form:

Subfield 1: The data numerator, an integer $<100$. We refer to this entry as $N_{1}$ in the following discussion.

Subfield 2: One of the special data operators listed below.

Subfield 3: A nine-character data entry, to be read in 79.0 format. It will be converted to an integer if the array is a " $\$$ " array or if a special array operator such as " $Q$ " is being used. Hote that an exponent is permissib?e but not required. Likewise, a decimal is permissitile but not required. If no decimal is supplied, it is assumed to be immediately to the left of the exponent, if any; and otherwise to the right of the last column. This entry is referred to as $N_{3}$ in the following discussion. 


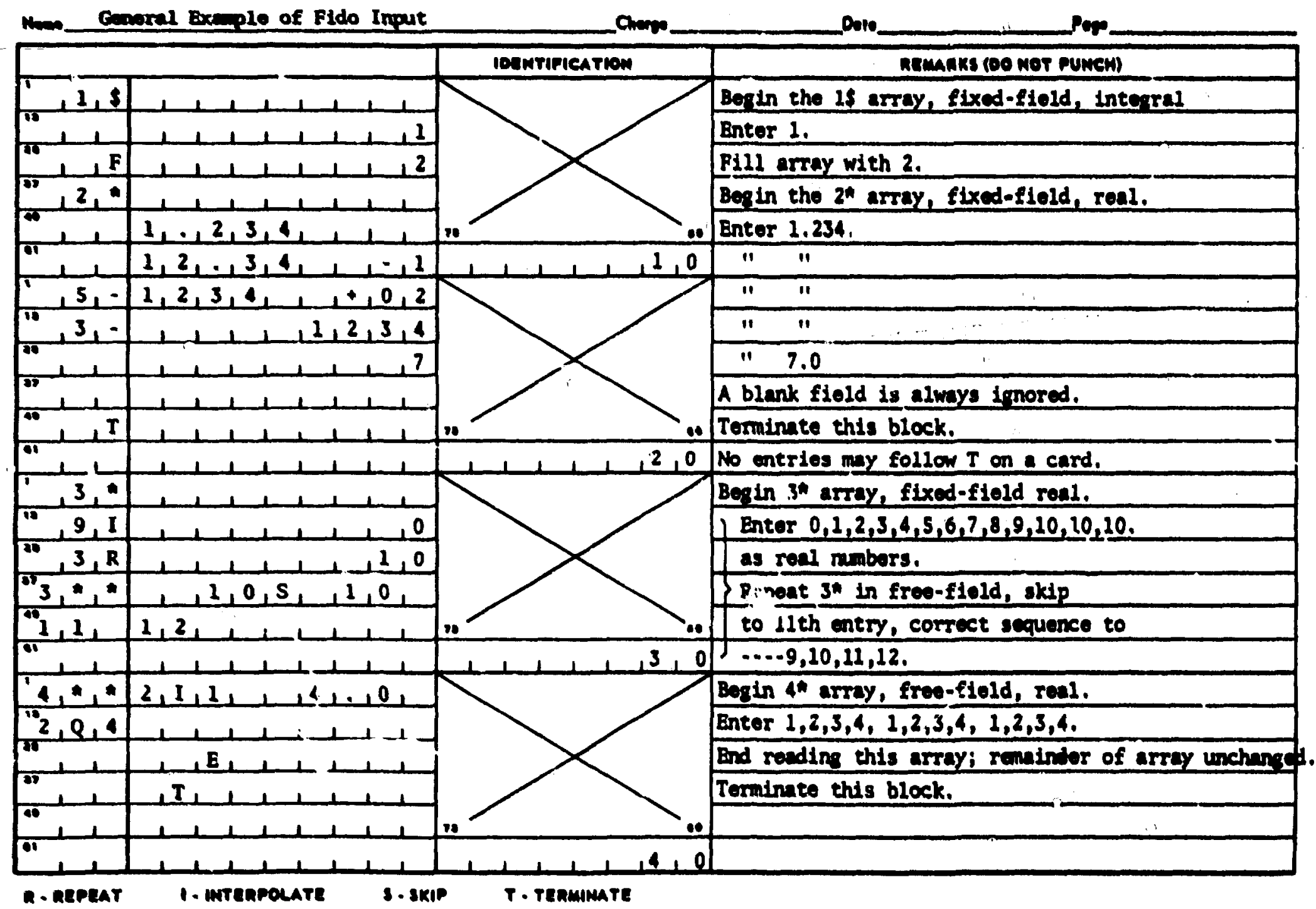


A list of data operators and their effect on the array being input follows:

"Blank" indicates a single entry of data. The data entry in the third subfield is entered in the location indicated by the pointer, and the pointer is advanced by one. However, an entirely blank field is ignored.

"f" or "-" indicates exponentiation. The data entry in the third field is entered and wultiplied by $10^{ \pm N_{1}}$, where $H_{1}$ is the data numerator in the first subfield, given the sign indicated by the data operator itself. The pointer is advanced by one. In cases where an exponent is needed, this option allows the entering of more significant figures than the blank option.

"g" has the same effect as "+".

" $R$ " indicates that the data entry is to be repeated $N_{1}$ times. The pointer is advanced by $N_{1}$.

"I" indicates linear interpolation. The data numerator, $\mathrm{N}_{\mathrm{I}}$, indicates the number of interpolated points to be supplied. The data entry in the third subfield is entered, followed by $\boldsymbol{x}_{1}$ interpolated entries equaliy spaced between that value and the data entry found in the thild subfield of the next non-blank field. The pointer is advanced by $\mathrm{N}_{1}+1$. The field following an "l" field is then processed normally, according to its own data operator. The "I" entry is especially valuable for specifying c spatial mesh. In "\$" arrays, interpolated values will be rounded to the nearest integer.

" $L "$ indicates logarithmic interpolation. The effect is the same as that of "I" except that the resulting dato are evenly separated in log-space. This is especially convenient for specifying an energy mesh.

"Q" is used to repeat sequences of numbers. The length of the sequence is given by the third subfield, $N_{3}$. The squence of $N_{3}$ 
entries is to be repeated $N_{1}$ times. The pointer is advanced by $N_{1} * N_{3}$. If either $N_{1}$ or $N_{3}$ is 0 , then a sequence of $N_{1}+N_{3}$ is repeated one time only, and the pointer is advanced by $N_{1}+N_{3}$. This feature is especially valuable for geometry specification.

The "I" option has the same effect as " $Q$ ", except that the order of the sequence is reversed each time it is entered. This is valuable for the type of symetry possessed by quadrature coefficients.

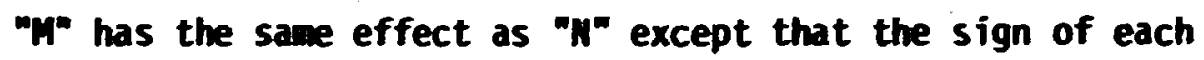
entry in the sequeice is reversed each time the sequence is entered. For example, the entries:

123242

would be equivalent to:

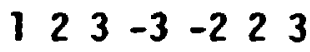

This option is also useful in entering quadrature coefficients.

" $Z$ " cáuses $N_{1}+N_{3}$ locations to be set to 0 . The pointer is advanced by $N_{1}+N_{3}$.

"C" causes the position of the last array item entered to be printed. This is the position of the pointer, less 1 . The pointer is not moved.

" 0 " causes the print trigger to be changr.d. The trigger is originally off. Successive " 0 " fields turn it on and off alternately. When the trigger is on, each card image is listed as it is read.

" $S$ " indicates that the pointer is to skip $N_{1}$ positions leaving those array positions unchanged. If the third subfield is nonblank, that data entry is entered following the skip, and the pointer is advanced by $N_{1}+1$.

"A" moves the pointer to the position $N_{3}$, specified in the third subfield. 
"F" fills the remainder of the array with the datun entered in the third subfield.

"E" skips over the remainder of the array. The array length criterion is almays satisfied by an "E", no matter how many entries have been specified. No more entries to an array may be given following an "E", except that data entry may be restarted with an " $A$.

The reading of data to an array is teninated wen a new array Jrigin field is supplied, or when the block is terminated. If an incorrect numer of positions has bren filled, an error edit is given, and a flag is set which will later aror: execution of the problen. FIDO then continues with the next array if an array origin was read. Otherwise, it returns control to the calling prograa.

A block termination consists of a field having " $T$ " in the second sub-field. All entries following " $T$ " on a card are ignored, and controi is returned from FIDO to the calling progran.

Coment cards can be entered within a block by placing an apostrophe (') in colum 1. Then columns 2-80 will be listed, with coltum 2 being used for printer carriage control. Such cards have no effect on the dara array or pointer.

Free-Field Input - With free-field input, data are written without fixed restrictions as to field and subfield size and positioning on the card. The options used with fixed-field input are available, although some are sliqhtly restricted in form. In general, fewer data cards are required for a probiem, the interpreting print is easier to read, a card listing is more intelligible, the cards are easier to keypunch, and certain common keypunch errors are tolerated withoit affecting the problem. Data arrays using fixed-and free-field input can be intermingled at will within a given block.

The concept of three subfields per field is still applicable to freefield input, but if no entry for a field is required, no space for it need be left. Only columns 1-72 may be used, as with fixed-field input. 
The array originator field can begin in any position. The array identifiers and type indicators are used as in fixed-field input. The type indicator is entered twice, to designate free-field input (i.e., "\$\$" or "t*"). The blank third stbfinld required in fixed-field ir.put is not required. For example: 31 tri indicates trat array 31 , a realdata array, will follow in free-field format.

Data fields may follow the array or:gin field imediately. The data field entries are identical to the fixed-field entries with the following restrictions:

(1) Any muber of blanks senjate fields, but at least one blank must follow a thiri subfield entry if one is used.

(2) If both first and second subfield entries are used, no blanks may separate then, i.e., 245, but not 24 S.

(3) Nuber; written with expunents must not have imbedded blanks, i.e., . $0 E+4,1.0 E 4,1.0+4$, or even $1+4$, but not 1.0 E4.

(4) In third-subfield data nntries, anly 9 digits, including the decimal but not includir., the expment field, can be used, i.e., 123456.89E07, but not 123456.789E $) 7$.

(5) The $Z$ entry must be of the forn: $338 Z$, not 2738 or $738 Z$.

(6) The + or - data operators are not needed and are not available.

(7) The $Q, N$, and $M$ entries are restricted: $3 Q 4,1 N 4$, or M4, but not $4 Q, 4 N$, or $4 M$.

User-field Input - If the user follows the array identifier in the array originator field with the character " $U$ " or " $V$ ", the input format is to be specified by the user. If " $U$ " is specified, the FORTRAN iORMAT to be used must be supplied in colums 1-72 of the next card. The format must be enclosed by the usual parentheses. Then the data for the entire array mist follow on successive cards. The rules of ordinary FORTRAN input as to exponents, blanks, etc., apply. If the array data do not fill the last card, the remainder must be left blank.

"V" has the same effect as "U" except that the format read in the last preceding " $U$ " array is used. 
CHAPTER XI. THE COVERX SERVICE MJDULE OF THE FORSS SYSTEM*

J. D. Prischler

*Originally published as ORNL/TM-7181. 
CONTENTS

Page

ABSTRACT .......................... v

I. INTRODUCTIOH .................... XI-3

II. COVERX FILE EXECUTION PATHS ................ XI-4

A. List .........................

B. Add ........................... XI-4

c. Delete ....................... XI-6

D. Merge ........................ XI-7

E. Format Conversion .................... XI-8

F. Record Modification ..................... XI-8

G. Edit/Copy ........................... $[\ldots 11$

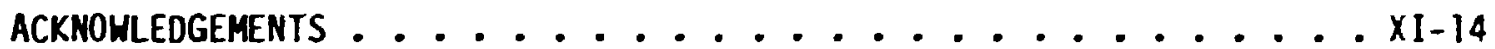

REFERENCES ..........................

APPENDIX A. THE FORMAT FOR STANDARD INTERFACE FILE COVERX FOR

MULTIGROUP CROSS-SECTION COVARIANCE MATRICES ......... XI-17

APPENDIX B. SAMPLE PROBLFM ........................ XI-23

B.1. JOB CONTROL CARDS FOR LOCAL USE AND SAMPLE INPUT FOR THE COVERX SERVICE MODULE .............. XI-25

B.2. SAMPLE OUTPUT FOR THE COVERX SERVICE MODULE ....... XI-27 
The COVERX Service Module includes seven execution paths to aid in understanding and uing multigroup cross-section covariance matrices contained in the standard interface file coVERX. The execution piths provide the follnwing operations on COVERX file(s):

1. List the contents of a COVERX file.

2. Allow adding new multigroup cross-section covariance matrices to an existirg COVERX file.

3. Allow deletion of multigroup covariance matrices from an existing COVERX file.

4. Merge two COVER: files and creates a new file.

5. Change the mode of a file from unformatted to formatted and conversely.

6. Allow modification of the records contained in a COVERX file.

7. Seleztively edits or copies a file. 


\section{INTRODUCTION}

Multigroup cross-section covariance matrices are created using the

PUFF cevariance file processing code ${ }^{1}$ and placed on a proposed $\operatorname{cccc}^{2}$ file, COVERX. ${ }^{3}$ Each covariance matrix is identified by the matrix control record of the COVERX file. The material and reaction type identification numbers are the MiT and MT numbers used in the ENDF/B $B^{4}$ files. Libraries 5 of multigroup matrices in COVERX format are currently available through the Enginearing Physics Information Center (ÉPIC) at Oak Ridge National Labo:atory and the National Nuclear Data Center (NNDC) at Brookhaven National Laboratory. The COVERX format is described in Appendix A. A sample problem utilizing the seven execution paths of the COVERX Service Module is described in Appendix B.

When the generation of a r.OVERX file(s) is complete for a study, a careful review is recommended before it is used. The COVERX service module is available to aid in this review. If identification problems or errors are detected, they can often be quickly corrected by the COVERX service module. At present, the module has seven execution paths which perform the following tasks:

1. Lists the contents of a COVERX $\mp$ ile.

2. Allows additional multigroup cross-section covariance matrices to be added to an existing COVERX file.

3. Allows deletions of multigroup cross-section covariance matrices from an existing COVERX file.

4. Merges two COVERX files and creates a new file.

5. Changes the mode of a file from unformatted to formatted and conversely. 


$$
X I-4
$$

6. Allows modification of the records contained in the COVERX file.

7. Selectively edits or copies a file.

The user selects the desired execution path by entering one of the following words on a card beginning in col um 1: LIST, ADD, DELETE, MERGE, CONVERT, FIX, EDIT. Additional FID06 input requirements are described for each execution path.

\section{COVERX FILE EXECUTION PATHS}

A. List

Input Data

LIST

$1 \$ \$$ Integer Parameter [1]

NBIN - Unit number of the COVERX file, if NBIN $<0$, only the matrix control records will be listed.

$T$

\section{B. Add}

This execution path allows multigroup cross-section covariance matrices to be added by creating a new file from the card input. The existing and newly sreated files are then mergad producing a new COVERX file.

Input Data

ADD

$1 \$ \$$ Integer Parameters [4] 
NOLD - Unit number of existing COVERX file

NADD - The number of coveriance matrices to be added

NEH - Unit nusber of new COVERX file

MSSS - Unit number of a scratch device (default = 31)

$T$

25\$ Integer Parameters [4]. The MATRIX CONTROL record of the COVERX file.

MAT1 - Material 1 ID

MT1 - Reaction type 1 ID

MAT2 - Material 2 ID

MT2 Reaction type 2 ID

$T$

3** Floating Point Parameters [NG]. The MATERIAL REACiION TYPE CROSS SECTION AND STANDARD DEVIATIONS record of the COVERX file. NG is the number of groups of the existing COVERX file. The standard deviations are automatically calculated by the code.

$(\operatorname{CRS}(\mathrm{J}), \mathrm{J}=1, N G)$

CRS - Cross sections (from $E_{\max }-E_{\min }$ )

T

[The $3^{* *}$ card is omitted if (MAT1.NE.MAT2.OR.MT1.NE.MT2)]

4\$\$ Integer Parameters [NG*2+1]. The BLJCK CONTROL record of the COVERX file.

Block control $=(\operatorname{JBAND}(J), I J J(J), J=1, N G),(\operatorname{LGRP}(N), N=1, N B L O K)$

For simplicity in creating the block control record of a covariance matrix, let NG equal the number of groups of the existing COVERX file and let NBLOK=1. The current version of TORSS $^{7}$ requires that NBLOK=1. 
Then: the $4 \$ \$$ card for a three-group problen with NBLOK=1 would be:

$4 \$ \$ 3132333$

OR: using the improved FIDO

$4 \$ \$ 01[\mathrm{NG}-1] \mathrm{Q} 2 \mathrm{Al}[\mathrm{NG}] 1 \mathrm{10}[\mathrm{NG}-1] \mathrm{Q} 2$ 09 [NG]

This is especially useful if matrices are large.

5* Floating Point Parameters [NG*NG]. The MATRIX DATA record of the COVERX FILE. If the preceding scheme for blocking is exercised, then the entire matrix can be input in one block. Cov - Matrix data (input by col.)

$\mathbf{T}$

The $2 \$ \$, 3^{\star \star}, 4 \$ \$$, and $5^{\star \star}$ sequence is repeated until NADD entries are complete.

\section{Delete}

This execution path allows multigroup cross-section covariance matrices to be deleted from a COVERX file.

Input Data

DELETE

$1 \$ \$$ Integer Parameters [3]

NBIN - Unit number of existing COVEnv file

NEW - Unit number of new COVERX file

NDEL - The number of covariances to be deleted 
$2 \$ \$$ Integer Parameters [4*NDEL]. The MATRIX CONTROL record of the COVERX FILE.

MATI - Material I ID

MT1 - Reaction type 1 ID

MAT2 - Material 2 ID

MT2 - Reaction type 2 ID

Sequence is repeated for each covariance to be deleted

$\mathbf{T}$

D. Merge

This execution path merges two existing COVERX files and creates a third file.

Input Data

MERGE

$1 \$ \$$ Integer Parameters [3]

N1 - Unit number of original COVERX file

If identical matrix control records are encountered on both the original files (N1) and the file to be merged (N2), the matrix found on unit (N2) is written on unit (N3), and the matrix found on unit (N1) is skipped. 


\section{E. Format Conversion}

This execution path implements features of the COVERT 8 code wich converts the COVERX file from informatted (binary) to formatted card images and conversely, and also lists a COVERX file.

\section{CONVERT}

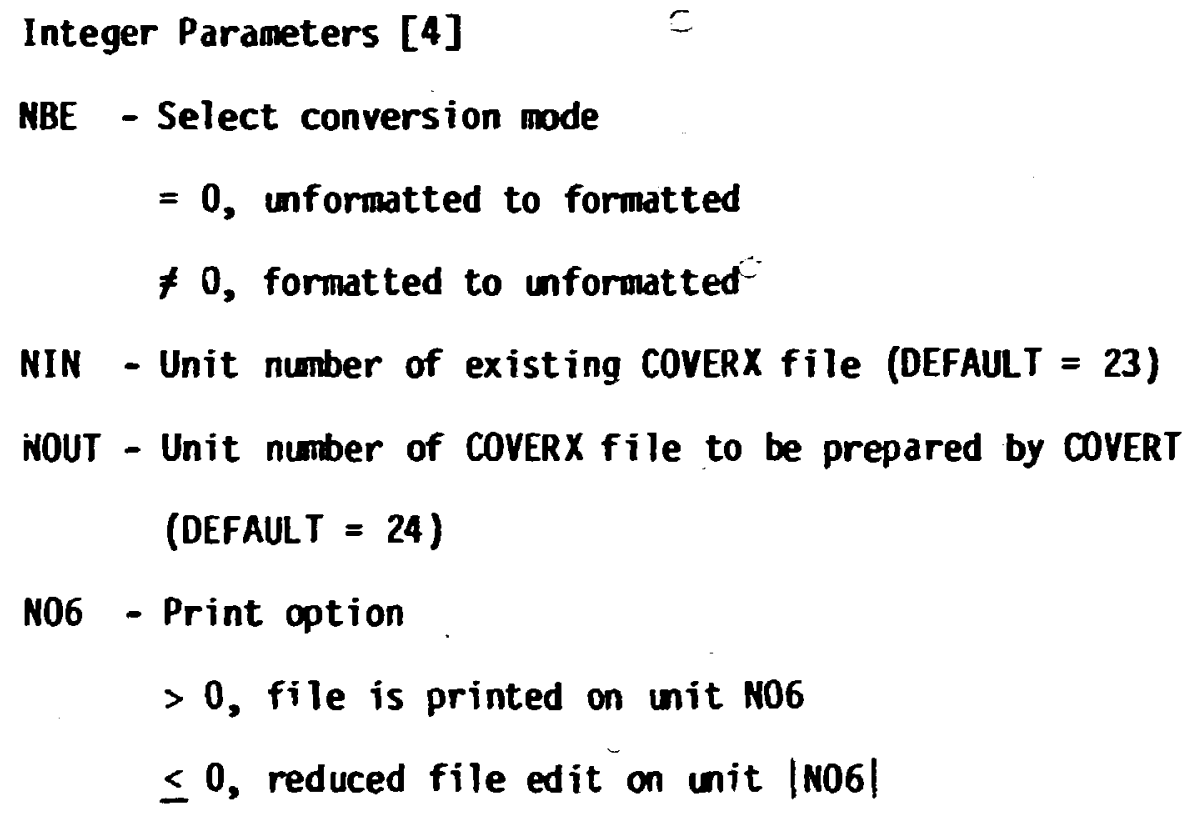

\section{F. Record Modification}

This execution path allows modification to tile records contained in a COVERX file. FIX modifies only those covariances which have been selected by previously using EDIT and wdates the existing COVFRX file by using MERGE.

Input Data

$F I X$

$1 \$ \$$ Integer "arameter [5] 
NOLD - Unit number of exisiing COVERX file

$>0$, complete listing

$<0$, reduced listing

NEW - Unit number of new COVERX file

NFIX - Number of covariance matrices to be reparied

MHOL - Modify file description record

$=0$, file description record nct modified

\#0, prepare 2f: card

USSS - Unit number of a scratch device (default = 31)

T

2ff Doubled Precision Parameters [200(A6) words max.]. The FILE DESCRIPTION record of the COVERX file (omit if NHOL $=0$ ). NUNIT - Unit number where Hollerith description of file is input NH - Number of (A6) words in Hollerith description

Then: using the improved FIDO

[NUNIT]G[NH] 6H(12A6) 6Hbbbbbb E T (b=blank) would al low NH (A6) words to be input from unit NUNIT.

$35 \$$ Integer Parameters [8]

NMATI - Material 1 ID

NMT1 - Reaction type 1 ID

MMAT2 - Material 2 ID

NMT2 - Reaction type 2 ID

NC? - Modify cross sections

$=0$, do not modify cross sections

* 0, prepare 4** card 


\section{$\mathrm{XI}-10$}

MMT - Modify matrix control record

$=0$, no modification

$\neq 0$, prepare $5 \$ \$$ card

NBLK - Modify block control record

$=$, no modification

f 0, prepare 6\$ card

NCOV - Modify matrix data record

$<0$, switch ruis and colimns

$=0$, no modification

$>0$, prepare $7^{\star \star}$ card

4 t* Floating Point Parameters [NG]. The MATERIAL-REACTION TYPE CROSS SECTIONS record of the COVERX file (omit if NCRS $=0$ ). NG is the number of groups of the existing COVERX file. The standard deviations are automatically calculated by the code.

$(\operatorname{CRS}(\mathrm{J}), \mathrm{J}=1, \mathrm{NG})$

CRS - Cross sections (from $E_{\max }-E_{\min }$ )

$T$

jss Integer Parame'ers [4]. The MATRIX CONTROL record of the COVERX

file (omit if NMT $=0$ ).

MATI - Material 1 ID

MT1 - Reaction type 1 ID

MAT2 - Material 2 ID

MT2 - Reaction type 2 ID

$5 \$ \$$ Integer Parameters [MG*2+1]. The BLOCK CONTROL record of the COVERX file (omit if NBLK $=0$ ). 


\title{
$\ddot{x i}-\mathbf{i j}$
}

\author{
Block control $=(J B A N D(J), I J J(J), J=1, N G),(L G R P(N), N=1, N B L O K)$ \\ JBAnD(J) - Band for group (J) \\ $[J J(J)$ - Position of diagonal element for grow (J) \\ LGRP(N) - Number of groups in block (N)
}

For simplicity in Ereating the block control record of a covariance matrix, let $N G$ equal the number of groups of the existing COVERX file and let MBLOK $=1$. The current version of FORSS requires that MBLOK $=1$.

Then: the 6\$\$ card for a three-group problem with NBLOK = 1 would be: 6553132333

OR: using the improved FIDO

$6 \$ \$ 01[N G-1] Q 2$ A1 $\mathrm{RG}] 1$ 19 [NG-1]Q2 $00[\mathrm{NG}]$

This is especially useful if matrices are large.

7*t Floating Point Parimeiers [NG*NG]. The MATRIX DATA record of the COVERX file (omit if $\mathrm{NCOV}=0$ ). If the preceding scheme for blocking is exercised, then the entire matrix can be input in one block.

cov - Matrix data (input by col.)

T

The $3 \$ \$$ through $7^{\star \star}$ sequence if repeated until NFIX entries are complete.

\section{G. File Edit/Copy}

This execution path permits selective listing and copying of a COVERX file. Each multigroup cross-section covariance matrix in a COVERX file is identified by the COVERX matrix control record. This record contains five 


\section{$X I-12$}

numers: Material 1 (MATl), reaction type 1 (MT1), Material 2 (MAT2), reaction type 2 (MT2), and the numer of blocks into hich matrix is subdivided (not used as input). Correlations between material-reaction types are extracted from the COYERX file using MTl, MII as the first waterialreaction type (colum designation), and MAT2, MT2 the second aterialreaction type (row designation).

As each covariance matrix is read from the COVER file, its first four identifiers are compared witk the corresponding identifiers in each edit comand. If equality is found between all of the identifiers in an edit comand and the corresponding identifiers from the watrix control record of the COVERX file, the multigroup cross-section covariance matrix qual ifies for the edit/copy operation.

Input Data

EDIT

$15 \$$

$$
\begin{aligned}
& \text { Integer Parameters [4] } \\
& \begin{array}{l}
\text { MBIN - Unit number of existing COVERX file } \\
\quad>0 \text {, complete listing } \\
\quad<0 \text {, file identification } \\
\text { NC - Number of edit commands } \\
\text { NEW - Copy option } \\
\quad>0 \text {, the unit number of a COVERX file to be written con- } \\
\quad \text { taining only those matrices edited } \\
\quad \leq 0 \text {, file not witten }
\end{array} \\
& \text { NSS - Unit number of a scratch device (default = } 31 \text { ) }
\end{aligned}
$$


255 Integer Parameters [4*in]

MIl - Material 1 ID

ITI - Reaction type I ID

MT2 - Material 2 ID

MI2 - Reaction type 2 ID

Complete MC edit comands

$\mathbf{T}$ 


$$
x 1-14
$$

ACKNOWLEDGEMENTS

The author wishes to acknowledge and thank J. L. Lucius,

J. H. Marble, and C. R. Meisbin for their useful suggestions and thorough review of this paper. Much of the important feedback, debugging, etc. was = obtained through discussions with J. D. Smith. Finally, deep gratitude is given to Sand Henry for her expert and patient typing of the several drafts of this report. 


\section{REFERENCES}

1. C. R. Heisbia, E. M. Oblow, J. Ching, J. E. White, R. Q. Hright, and J. D. Drischler, "Cross Section and Method Uncertainties: The Application of Sensitivity Analys is to Study Their Relationship in Radiation Transport Benchmark Problems," ORML/TM-4847 (ENOF-218) (August 1975); see also RSIC Code Collection PSR-93.

2. B. M. Carmichael, Standard Interface Files and Procedures for Reactor Physics Codes, Version III, LA-5486-MS (February 1974).

3. 1. L. Lucius and C. R. Meisbin, "Interface Specifications for Sensitivity Profiles (SENPRO) and Covariance Files (COVERX)," Presentation to the Comittee on Computer Code Coordination, Los Alamos Scientific Laboratory (May 1970).

4. M. K. Drake, Data Formats and Procedures for the ENDF Meutron CrossSection Library, Brookhaven National Laboratory report BHL -50274 (April 1974 Revision).

5 J. D. Drischler and C. R. Heisbin, "Compilation of Multigroup CrossSection Covariance Matrices for Several Important Reactor Materials," ORNL-5318 (ENDF-235) (October 1977).

6. J. H. Marable, "The Improved FIDO Input System," Handout G of materials used in the RSIC Horkshop on the ORNL FORSS Sensitivity and incertainty Analysis Code System (August 1978).

7. C. R. Heisbin, J. H. Marable, J. L. Lucius, E. M. Oblow, F. R. Mynat:, R. H. Pet!le, and F. G. Perey, "Application of FORSS Sensitivity and Uncertainty Methodology to Fast Reactor Benchmark Analys is," ORNL/TH-5563 (1976).

8. J. D. Drischler, J. H. Marable, and C. R. Weisbin, "COVERT and CAVALIER: Two Computer Codes for Estimating Uncertainties of Calculated Neutronics Parameters Using Standard Interface Files SENPRO and COVERX," ORNL/TM-6078 (ENDF-256) (August 1978). 


$$
x !-! ?
$$

\section{APPENDIX A}

The Fo: mat for Standard Interface File COVERX for Multigroup Cross-Section Covariance Matrices 


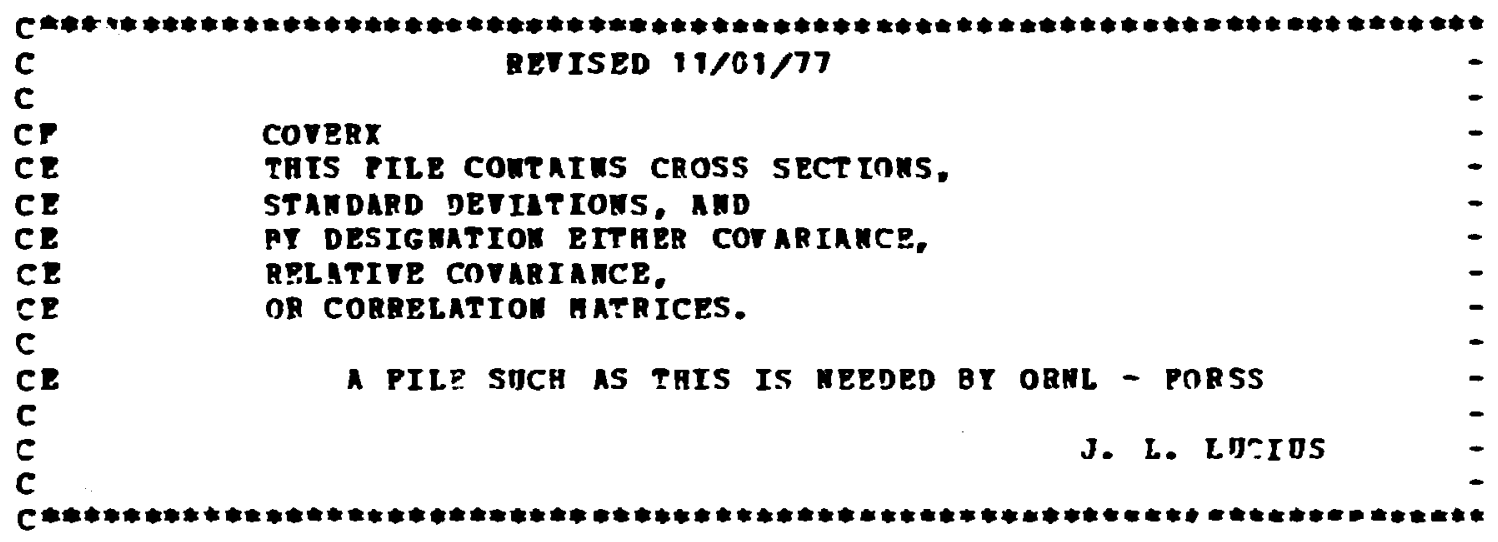

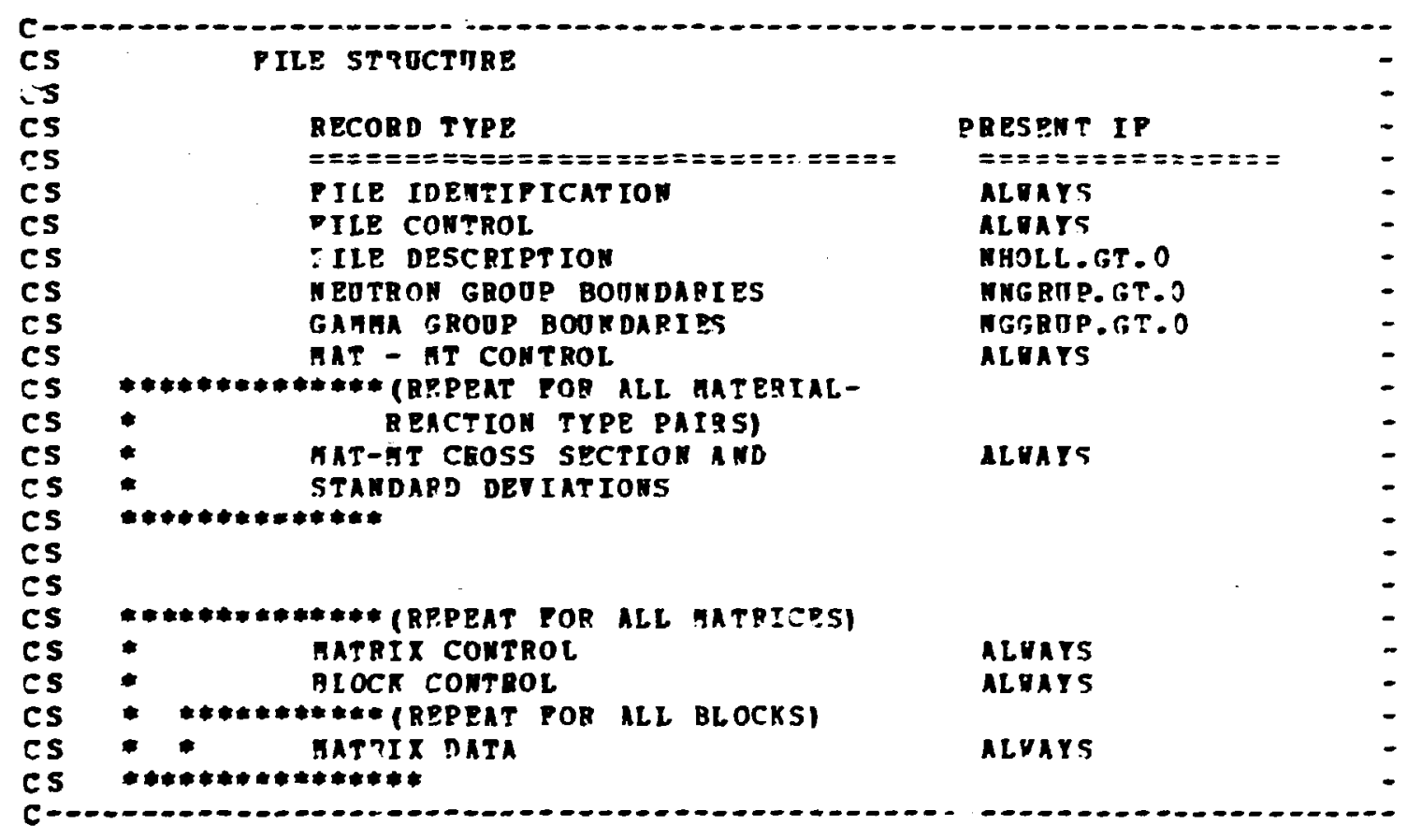

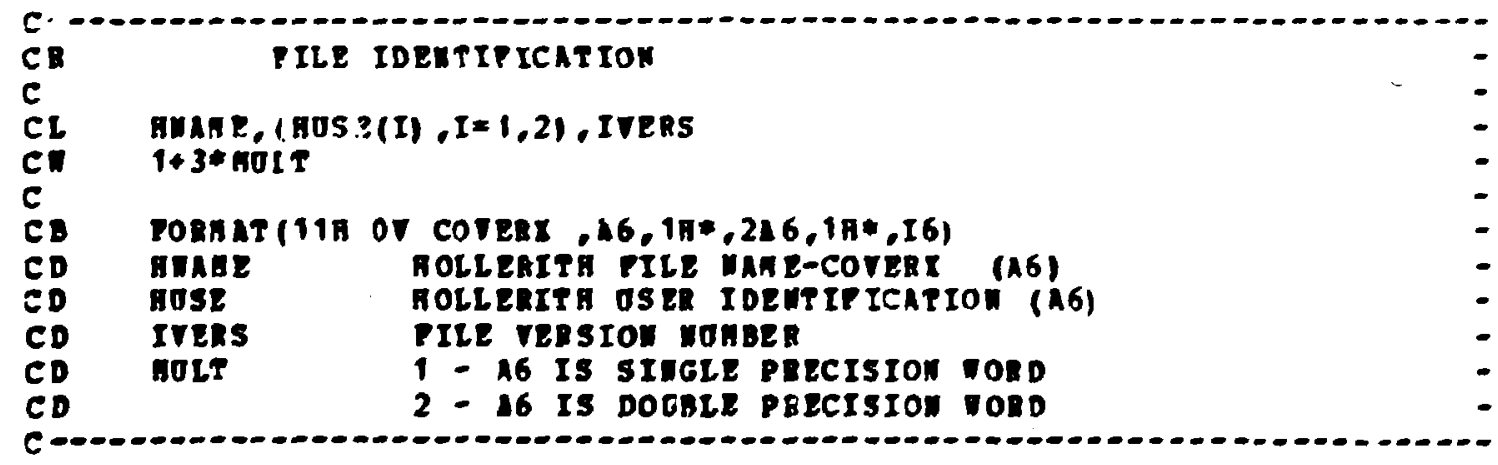




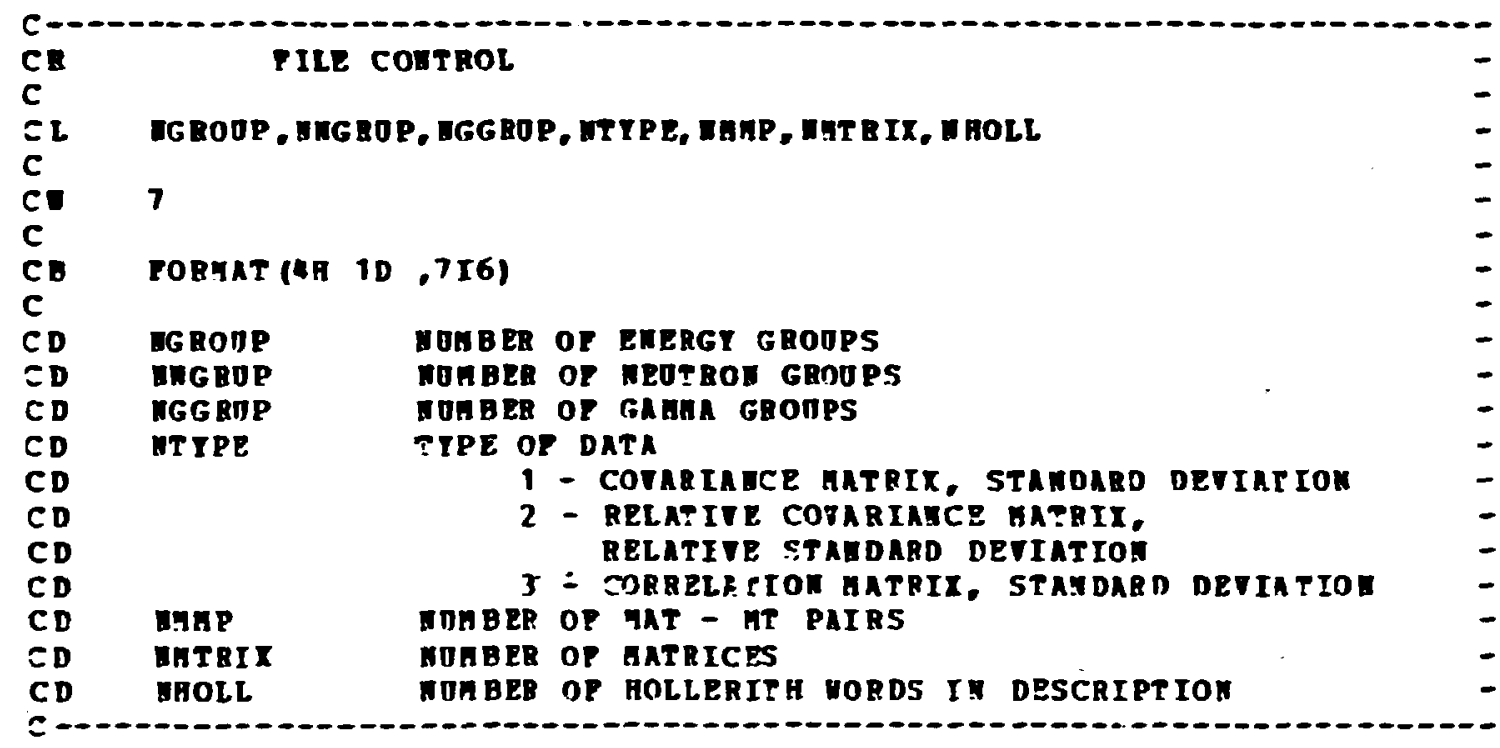

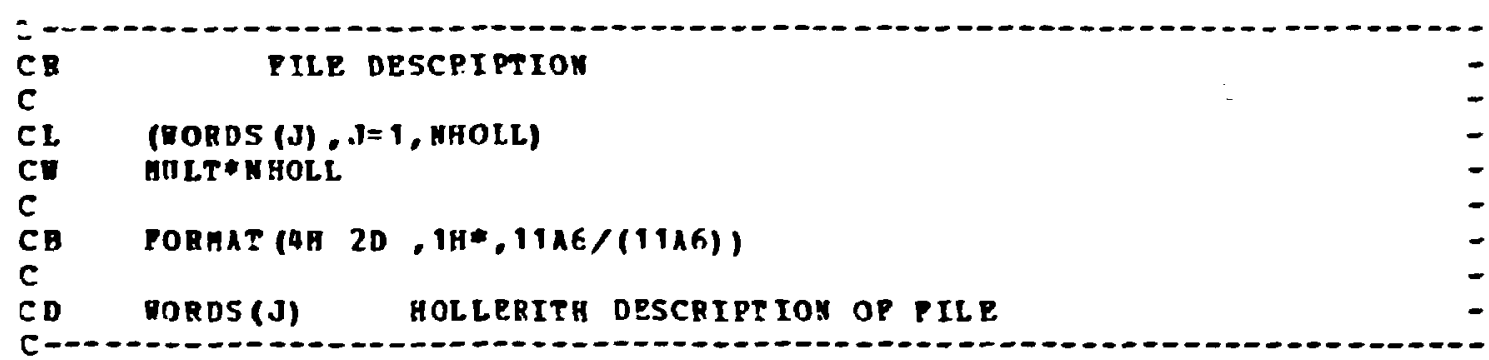

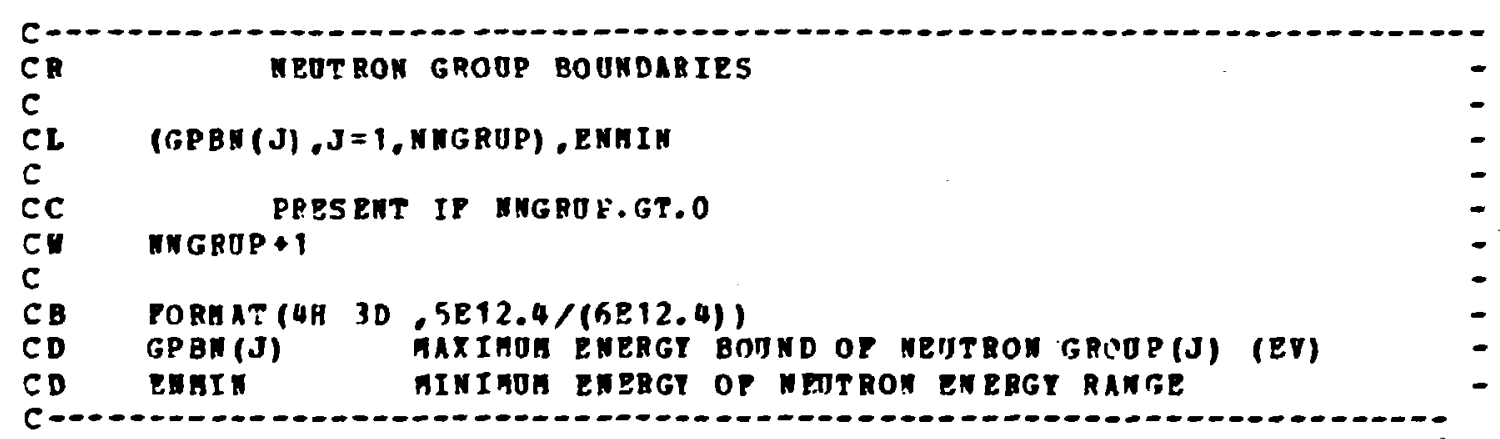

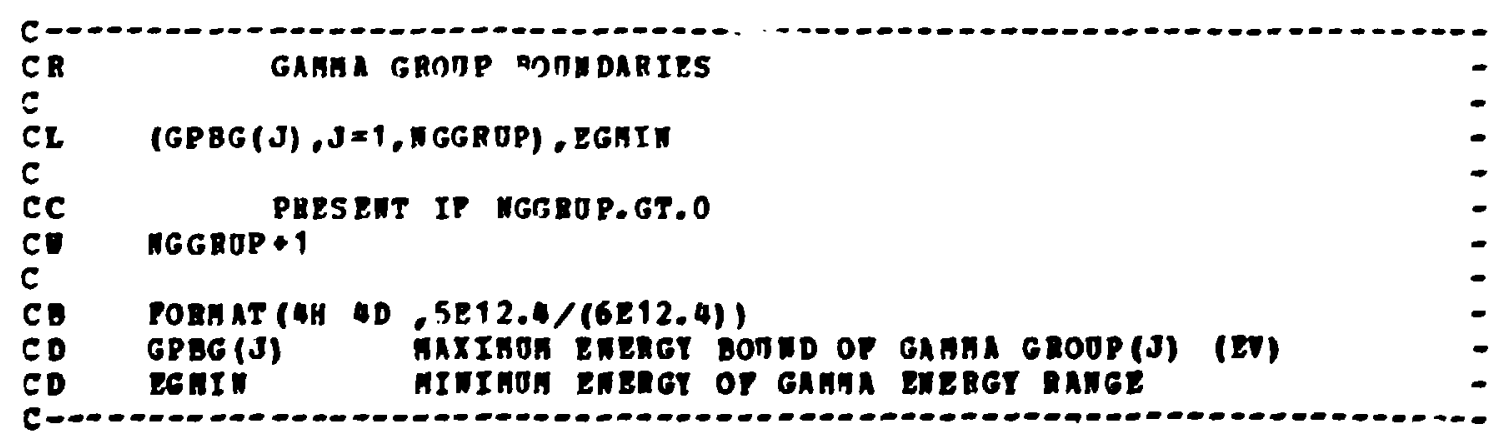




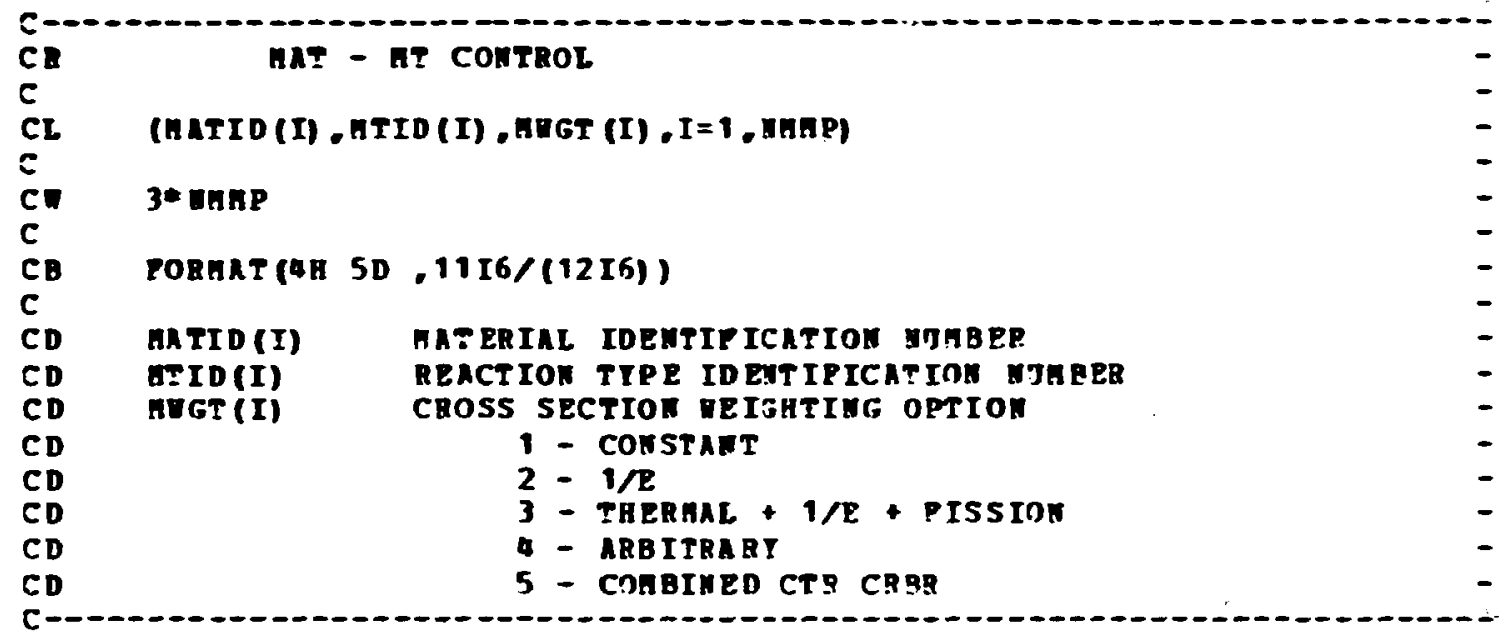

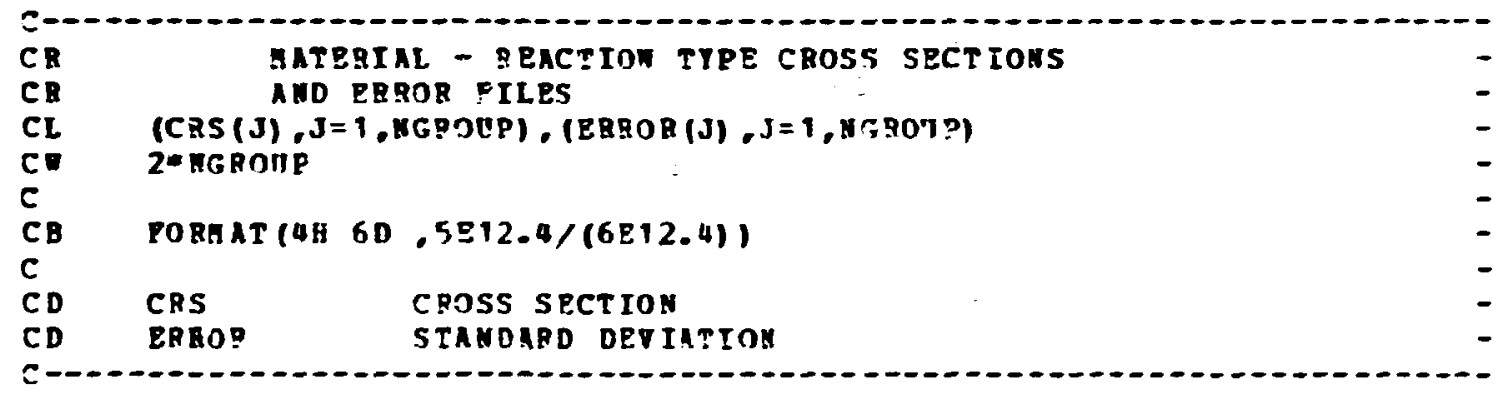

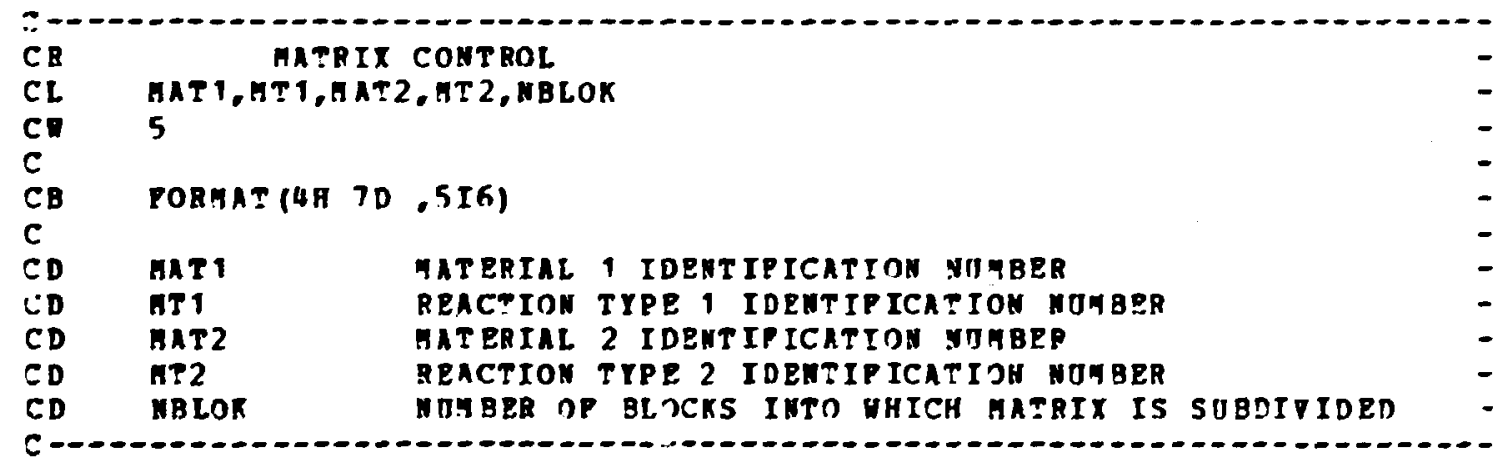

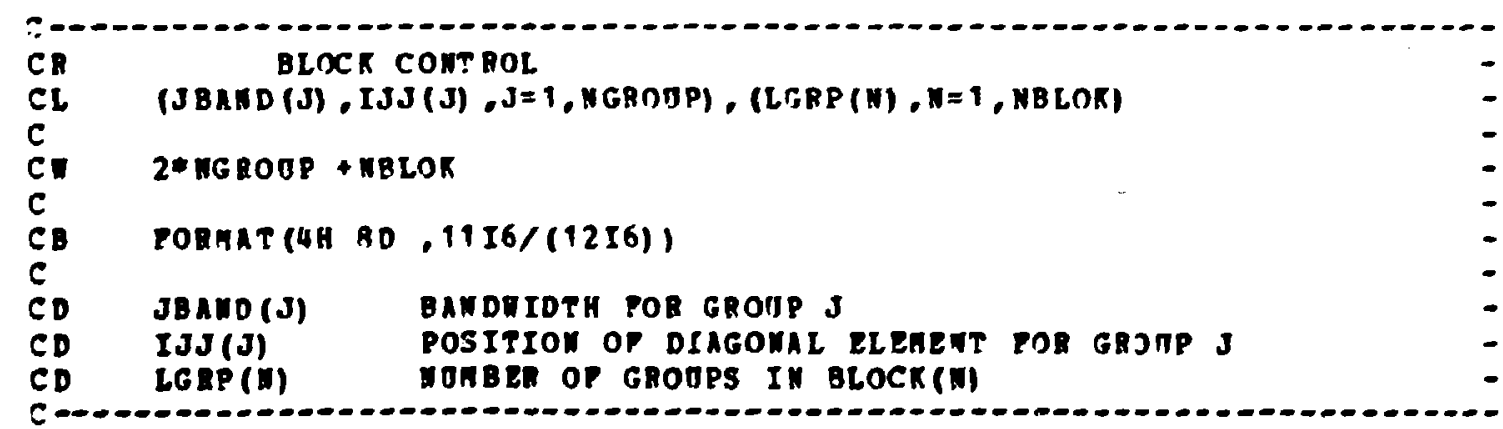


$X I-21$

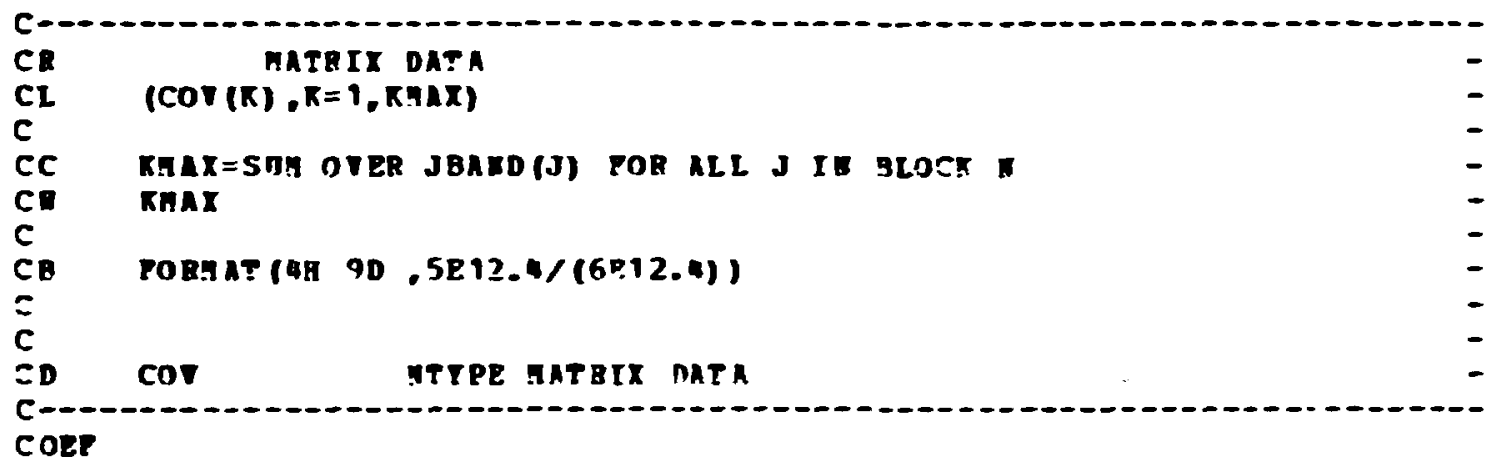


APPENDIX B.

Sample Problem

The following sample input and output problem utilizes the seven execution paths available in the COVERX Service Module. The user should be aware that although all the execution paths are utilized in the sample problem, the input parameters may vary depeilding on the user's requirements.

A description of the input to the sample problem follows:

1. CONVERT - The formatted card image COVERX file residing on unit 24 is converted to a binary file on unit 32 , and the resulting file is listed using the reduced file edit option.

2. EDIT - The $235 U(n, f)$ covariance matrix is extracted from the binary COVERX file and copied to unit 33 creating a new COVERX file containing one covariance matrix. The output for EDIT gives a complete listing of the COVERX file.

3. FIX - The $235 U(n, f)$ covariance data residing on unit 33 is changed as follows:

The Hollerith description of the file is modified (2kit),

The cross sections were modified $\left(4^{\star \star}\right)$,

The matrix control record was modified renaming the $235 u(n, f)$ covariance data (5\$\$),

The block control record was modifted (6\$\$), and The covariance data was modifted (7**). 
The output of $F I X$ resides on unit 34 , and a complete listing of the input plus all modifications is given.

4. EDIT - Three additional covariance matrices were extracted from unit 32 and copied to unit 35.

5. MERGE - The COYERX file residing on unit 35 and the one residing on unit 34 are merged to produce a single COVERX file on unit 33 containing four covariance matrices.

6. DELETE - The covariance matrix indicated in the $2 \$ \$$ array is deleted from unit 33 , and the remaining covariances are placed on unit 34 .

7. ADD - A new covariance indicated in the $25 \$$ array was added to the COVERX file residing on unit 34 with incl usion of the Block control record (4\$\$) and covariance data (5*t). This produced a new COVERX file on wit 33. 8. LIST - The entire COVERX file residing on unit 33 was listed. 
APPENDIX B.1. Job Control Cards for local Use and Sample Input for the COVERX Service Module

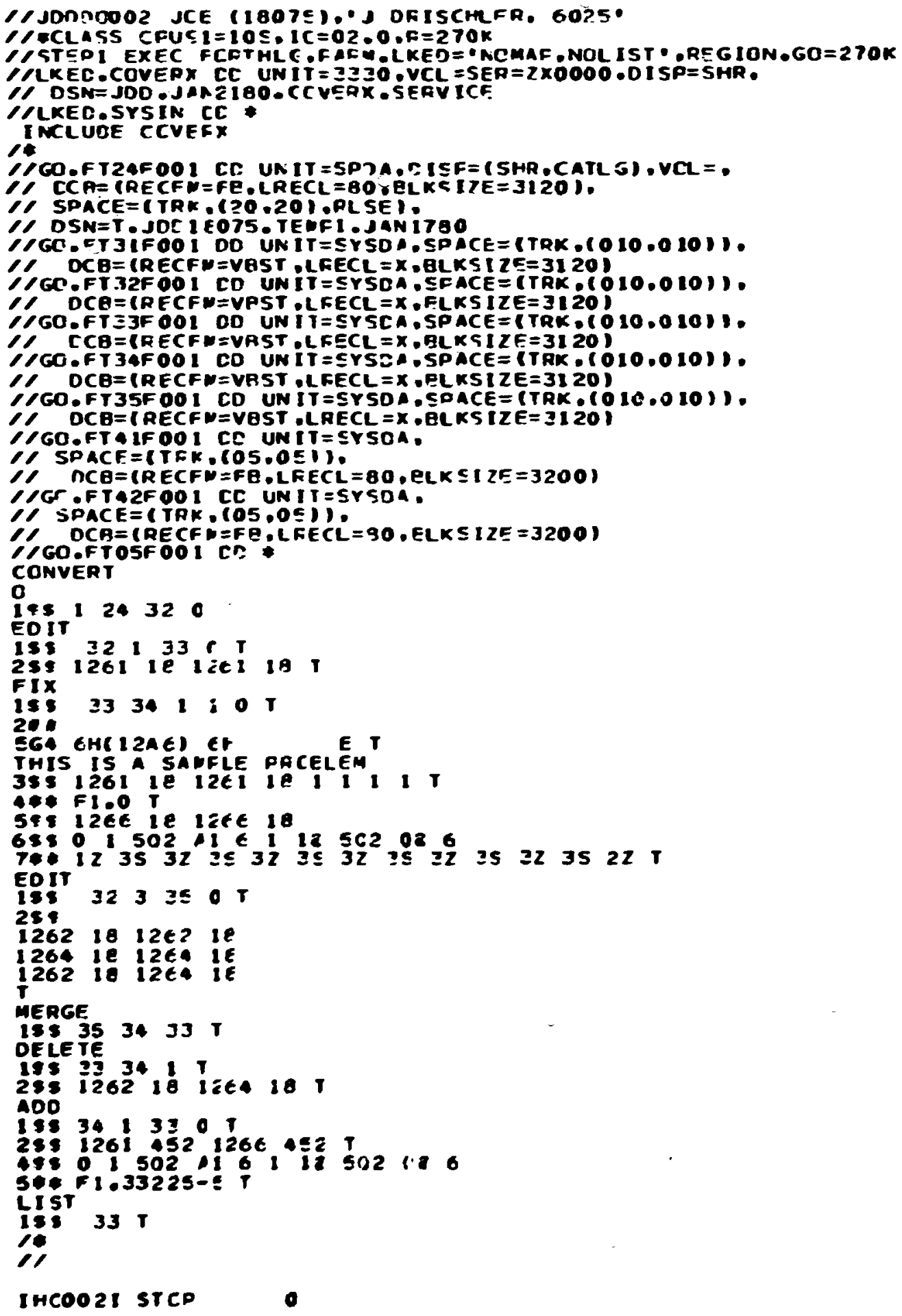




$$
\times 1-2 ?
$$

APi-NDIX B.2. Sample Output for the COVERX Service Module 


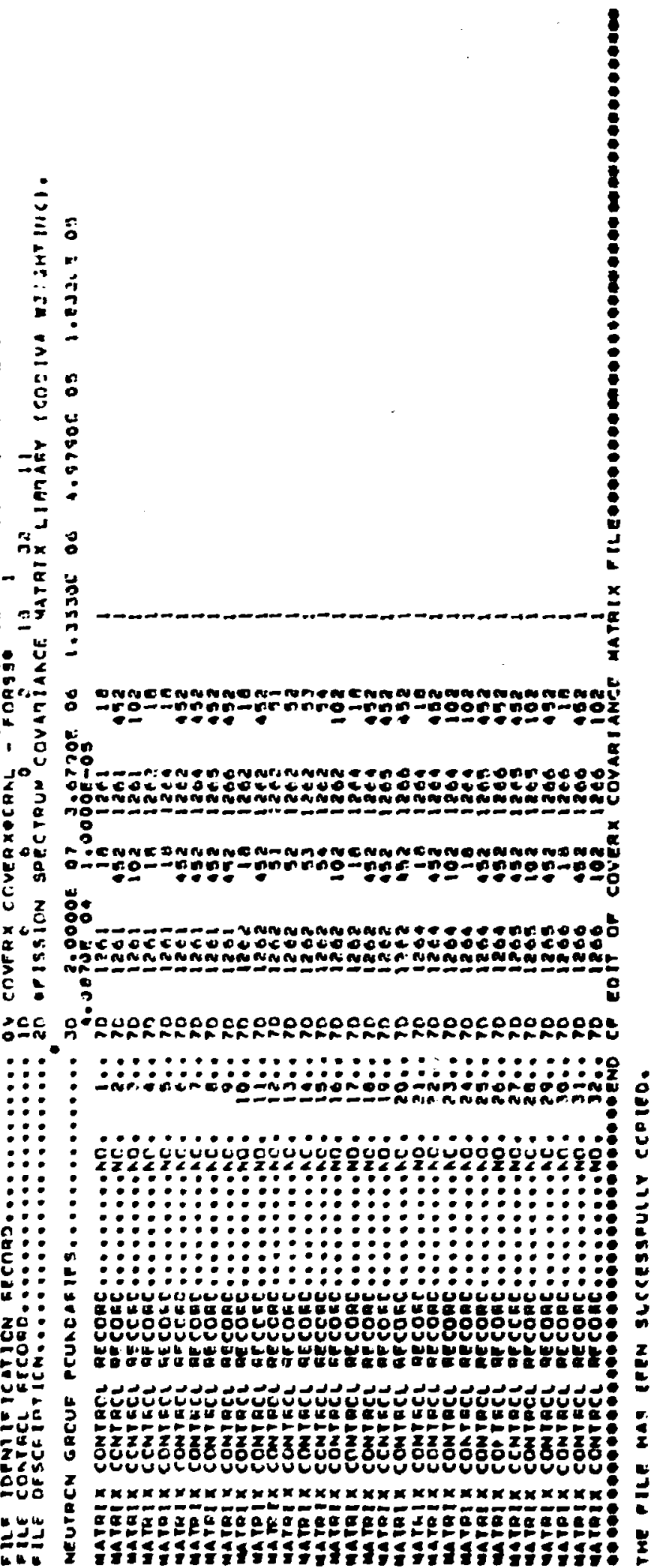




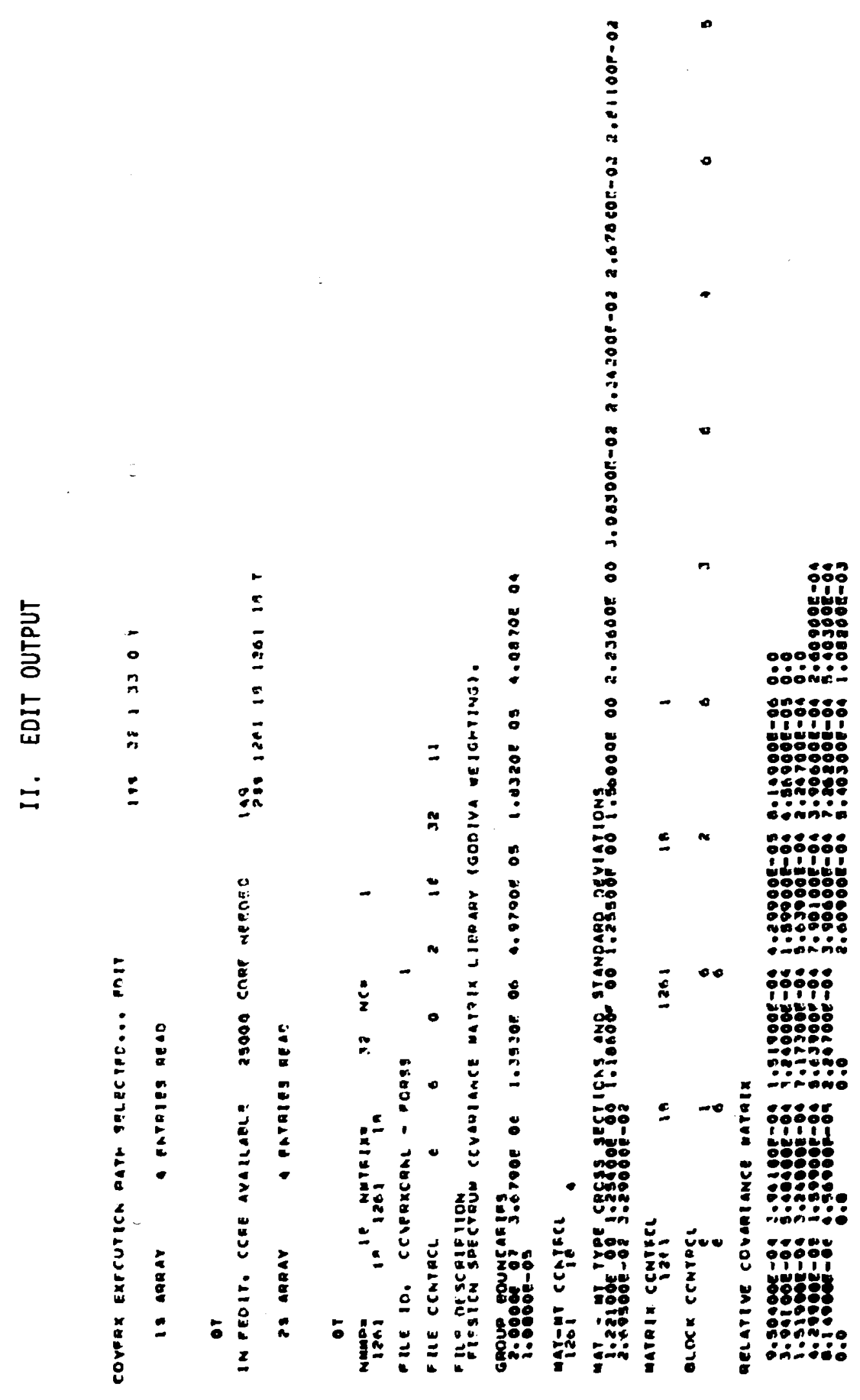


III. FIX OUTPUT

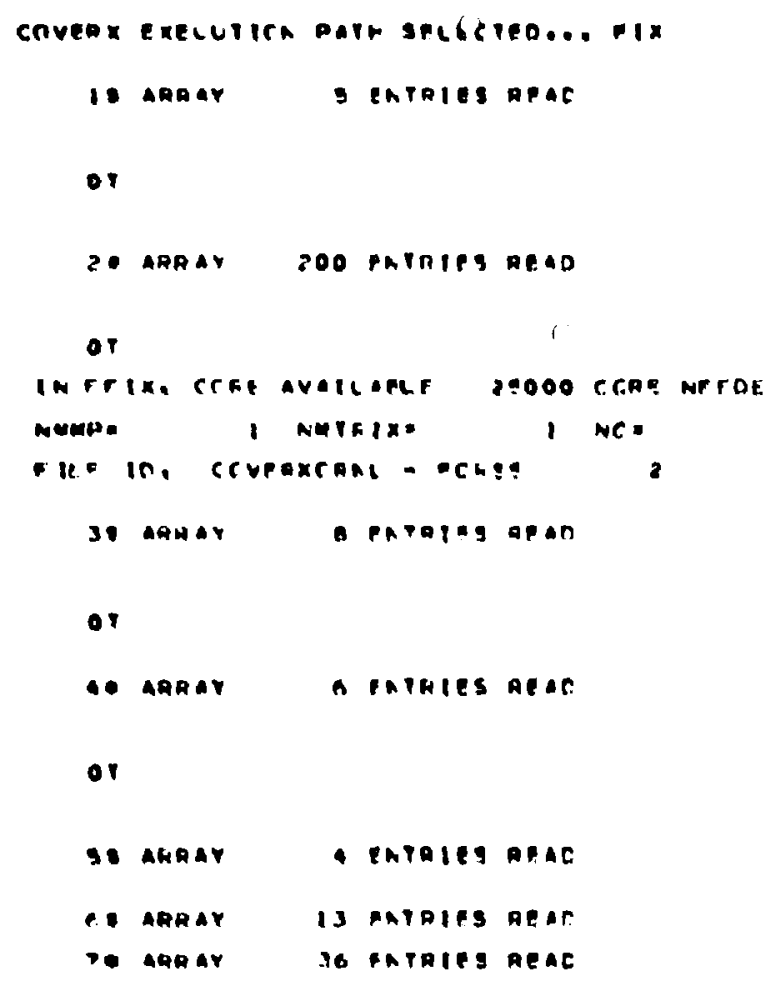

\author{
12133341109
}

EQG OR(18AG) OM T

113

3812011012011011819

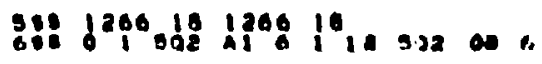

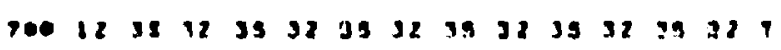


III. FIX OUTPUT (cont'd.)

$$
\text { or }
$$

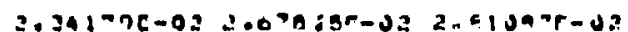


IV. EDIT OUTPUT

$118 \quad 32335001$

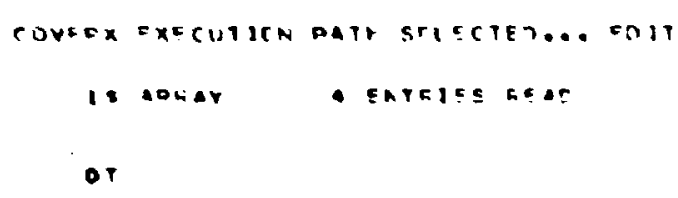

Pasact

15 Fathied akan

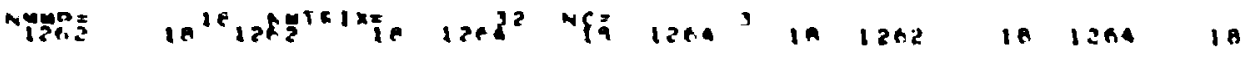

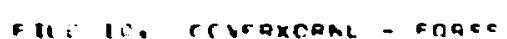

1

- lie contare

$a \quad 0$

5

le

3. 11

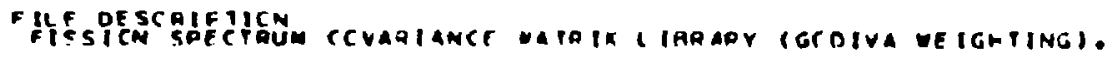

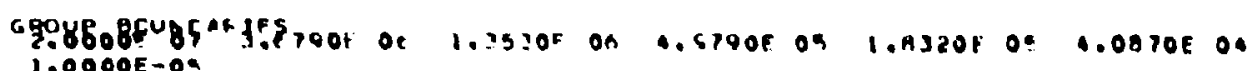

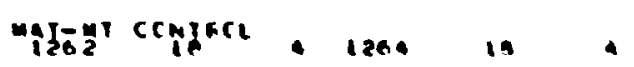

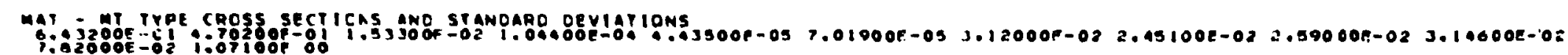
然 matain cepparel

acoer coninge

$\begin{array}{rr}19 & 1262 \\ \vdots & \vdots\end{array}$

\begin{abstract}
in
$?$
\end{abstract}

6

-

(

$x$ 
Relative covariance mateix

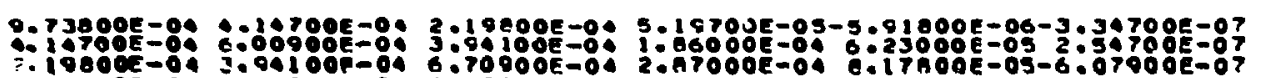

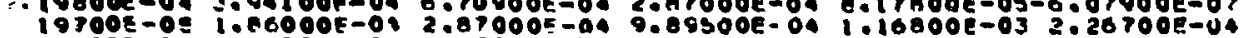

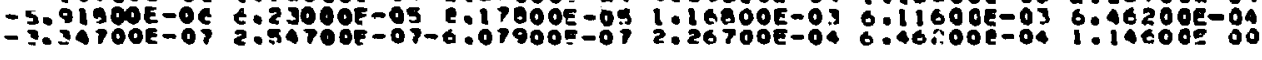

matRIX CCMTECL

1264

10

BI =K CCNTAgE

e

2

6

RELATIVE COVARIANCE matwlx

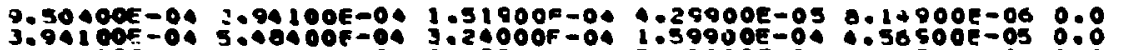

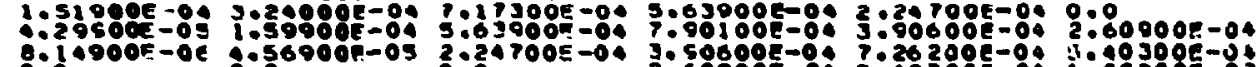
matrix centegl

BLCCK CONIRCL

is

1260

18

8

2

1

Relative cCVARIANCF matalx

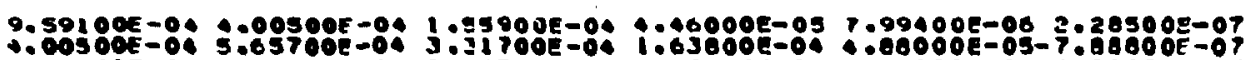

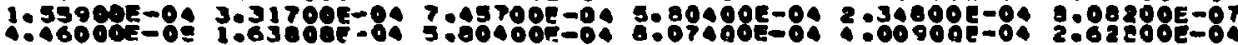

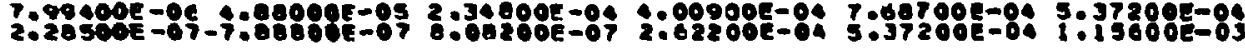




\section{MERGE OUTPUT}

COVERX EXECUTICN PATH SELECTEC... MERG

15 ARRAY 3 ERTRIES REAC

\section{or}

IN FMERG, CCFE AVAILAELE

MERGE 35 IS

IFC $6 \quad 6$

JFC $1262^{6} 61 e^{6}$

$\begin{array}{lllll}0 & 2 & 2 & 3 & 11\end{array}$

e
$21124^{1} 418$
$158 \quad 3534 \quad 33 \%$

渵

190 
VI. DELETE OUTPUT

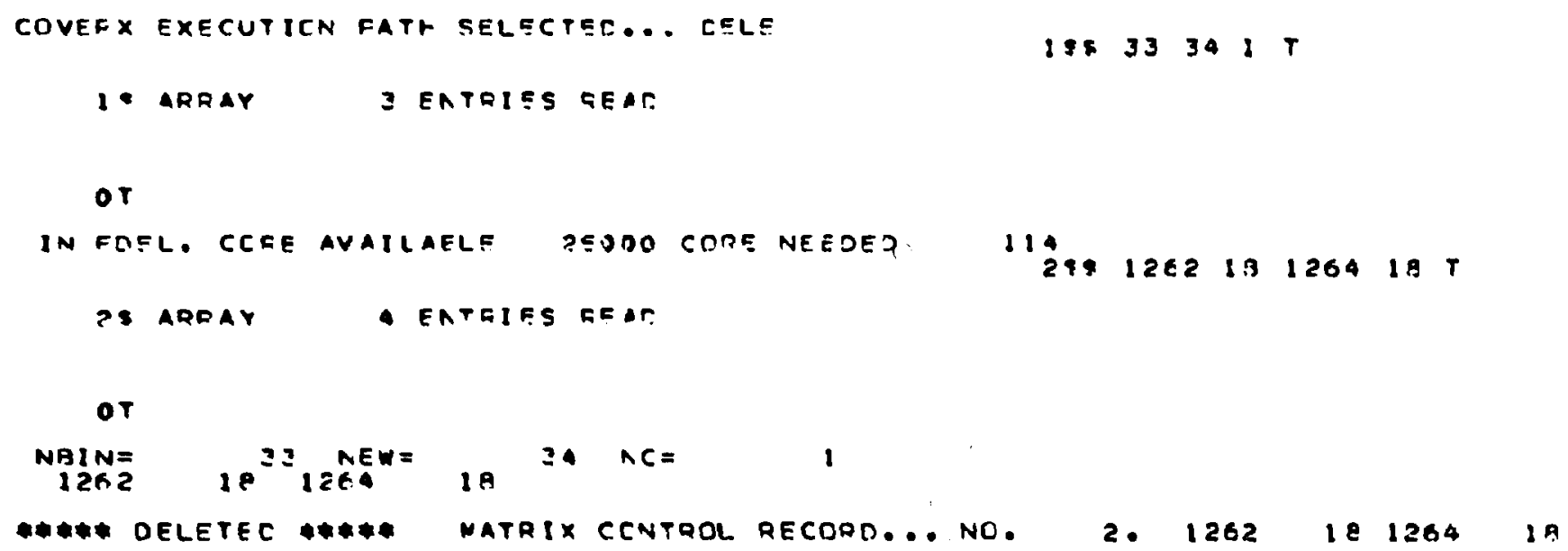

ot

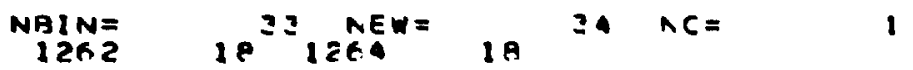

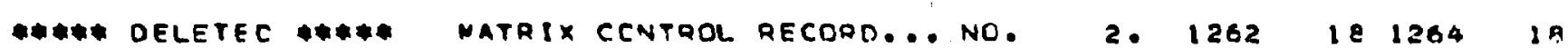




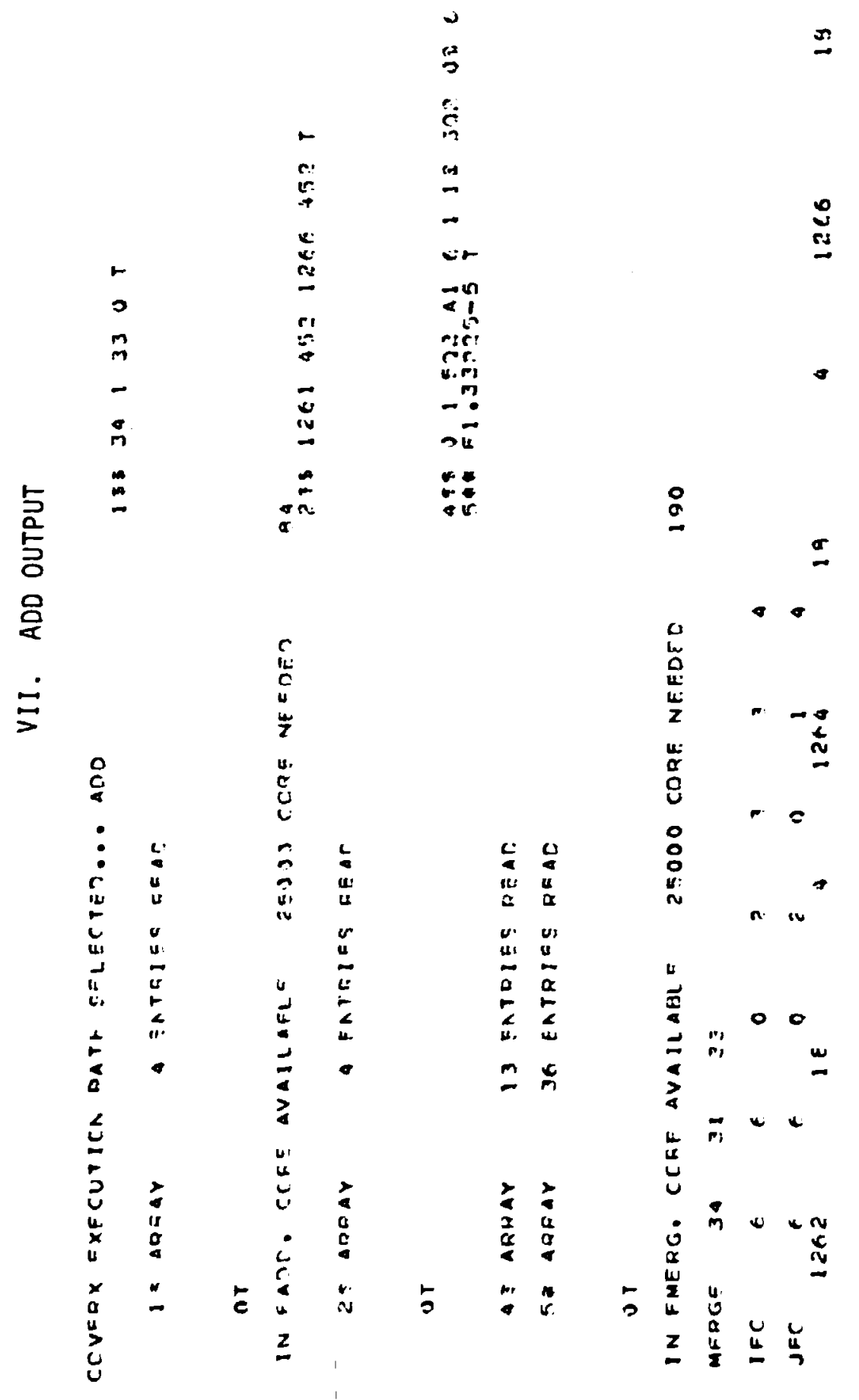


XI-3?

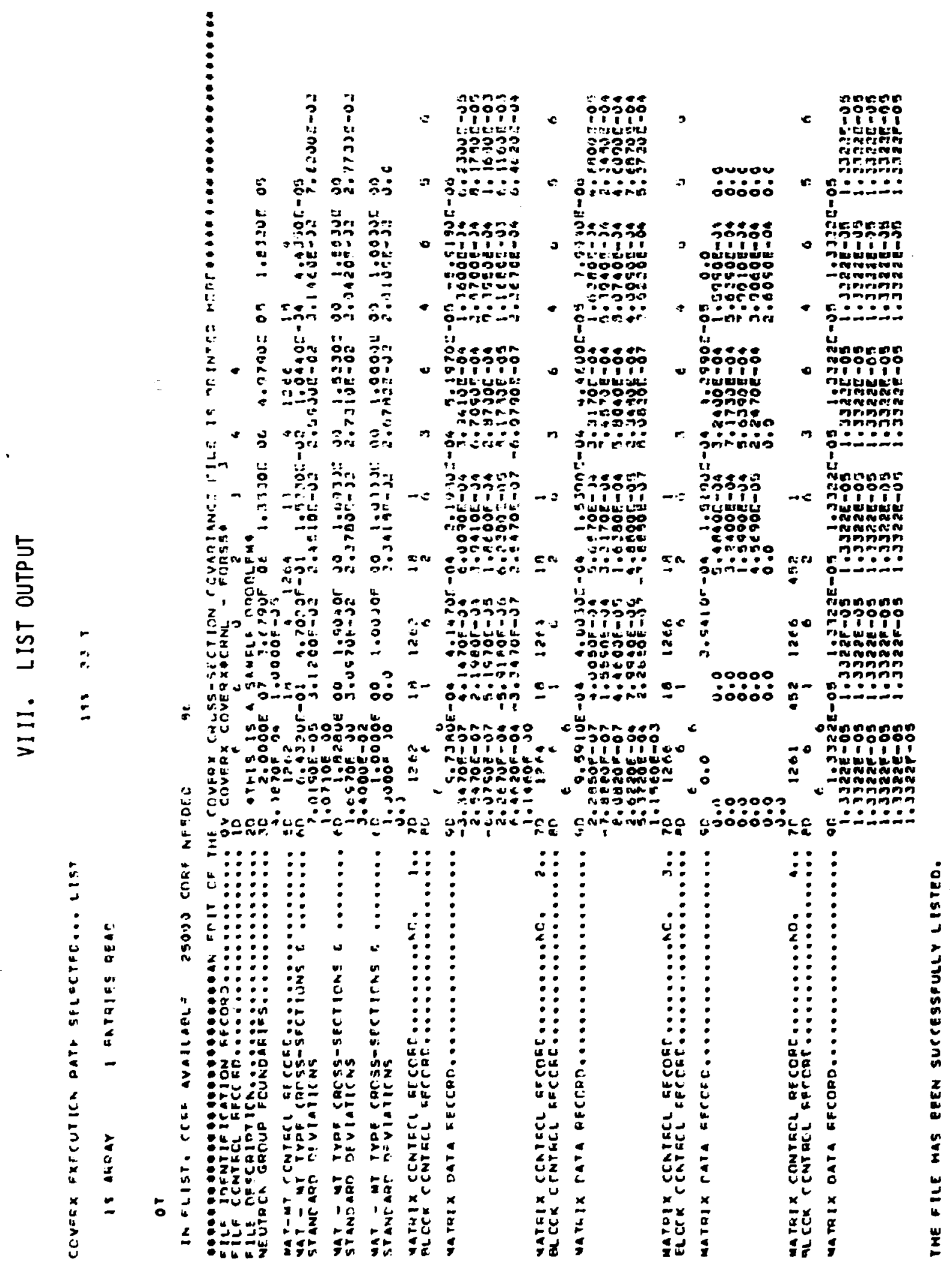


XII. THE UNCOVER MODULE OF THE FORSS SYSTEM

J. H. Marable

J. L. Lucius 
General Development................. xII-3

Uncover Input Preparation ............. XII-5

Uncover Input ................... XII-9

Comments on the $22^{\star \star}$ Card .............. $x_{11-12}$

Comments on the $0 \$ \$$ Array ................ XII-13

Comments on the $23 \$ \$$ Array ............ XII-14

Output Discussion ................. XII-16

Uncover Error Stops ................. XII-18

Resource Utilization ................ xLI-19 
THE UNCOVER MODLLE

UNCOVER is the FORSS module that incorporates the data from integral experiments with the evaluated nuclear data based on differential crosssection measurements and prepares data files which are used to adjust (see Chapter XIV) the nuclear data base leading to improved performance parameter prediction. UNCOVER requires sensitivity coefficients and covariance matrices as input. This data may be input by a card input stream or via SENPRO and COVERX files. A convenience module COVERS (see Chapter XIII) is available to aid in UNCOVER input preparation when SENPRO and COVERX files are used.

\section{GENERAL DEVELOPMENT}

The least-squares adjustment formula gives the minimum variance estimates $x^{-}$of experimentally determined quantities $x^{e}$ with covariance $B$. It is assumed that the estimates $x$ - are subject to linear constraints imposed by theory and expressed as

$$
S\left(x^{-}-x^{c}\right)=0
$$

where $x^{c}$ is any set of values consistent with the theory. The representation of the constraints by a matrix $S$ is not unique since any linear equation remains a valid linear equation when multiplied by an arbitrary nonzero constant

The least-squares values for $x^{-}$are given by

$$
x^{-}-x^{e}=p_{1}\left(x^{c}-x^{e}\right)
$$

where $P_{1}$ is a projection operator given by

$$
P_{1}=B S^{\top}\left(S B S^{\top}\right)^{-1} \mathrm{~S} \text {. }
$$

The super-f indicates the transpose. 
The covariance associated with these adjusted values $x^{-}$is given by

$$
\begin{aligned}
B^{-} & =\left(1-P_{1}\right) B\left(1-P_{1}\right)^{\top} \\
& =B-P_{1} B .
\end{aligned}
$$

In application it is convenient to partition the matrices $x^{e}, x^{C}, B$, and $S$ into submatrices; $x^{\prime}, P_{1}$, and $B^{\prime}$ are also partitioned accordingly.

$$
\begin{aligned}
& x^{e^{\top}}=\left[\begin{array}{llll}
x_{\alpha}^{e} & x_{\beta}^{e} & \ldots & x_{\gamma}^{e}
\end{array}\right] \\
& x^{c^{\top}}=\left[\begin{array}{llll}
x_{\alpha}^{c} & x_{B}^{c} & \ldots & x_{\gamma}^{c}
\end{array}\right] \\
& S=\left[S_{\alpha} S_{B} \ldots S_{\gamma}\right]
\end{aligned}
$$

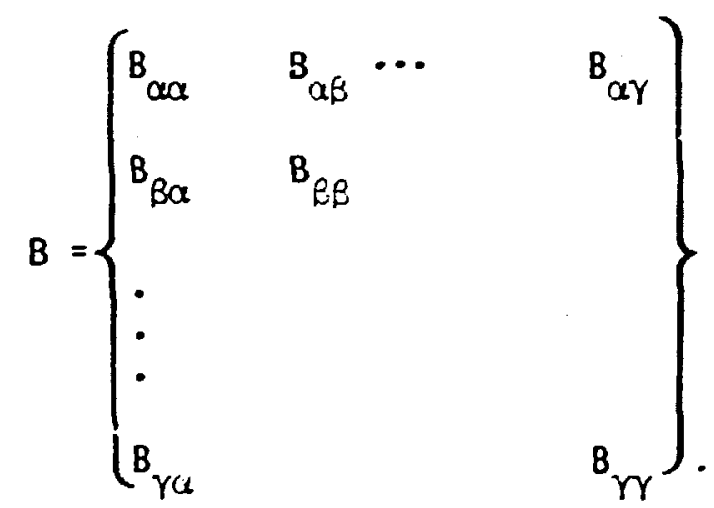

The partitions and the form of $S$ can be chosen so that $S_{\alpha}$ is the negative of a unit matrix. This form is assumed in the code UNCOVER, and accordingly $S_{\alpha}$ is nct required in the input. The remaining $S_{e} \cdots S_{\gamma}$ are then ordinary sensitivity coefficients. The code also assumes submatrices $x_{B}^{c} \cdots x_{\gamma}^{c}$ are chosen to be equal to submatrices $x_{\beta}^{e} \cdots x_{\gamma}^{e}$. Accordingly the input only requires the first submatrix of its difference $\left(x_{\alpha}^{c}-x_{\alpha}^{e}\right)$ since the other partitioned submatrices have zero differences.

The chi-square of the adjustment is given by

$$
\begin{aligned}
x^{2} & =\left(x^{-}-x^{e}\right)^{\top} B^{-1}\left(x^{-}-x^{e}\right) \\
& =\left(x^{c}-x^{e}\right)^{\top} S^{\top}\left(S B S^{\top}\right)^{-1} x\left(x^{c}-x^{e}\right)
\end{aligned}
$$


and components of $x^{2}$ are found from

$$
x_{\alpha \beta}^{2}=\left[\left(x^{c}-x^{e}\right)^{\top} S^{\top}\left(S B S^{T}\right)^{-1} S\right]_{\alpha{ }_{\alpha \beta}^{B}}\left[S^{\top}\left(S B S^{T}\right)^{-1} S\left(x^{c}-x^{e}\right)\right]_{\alpha}
$$

These components sum to $x^{2}$.

\section{UNCOVER INPUT PREPARATION}

The input data for code module UNCOVER is based on the partitioning of the extended covariance matrix into NPxNP submatrices and the part:tior.ing of the sensitivity coefficient matrix into (NP-1) XNC submatrices. This partitioning is defined by two partitions; the first partition is defined by NP integer parameters and the second partition is defined by NC integer parameters. The present restrictions on these partitions are (1) that NP be greater than or equal to 2 and not greater than $50,(2)$ that $N C$ be equal to 1 , and (3) that the first integer parameter of each partition be the number of integral experiments NIE. Thus, the first partition is a set of NP integer parameters whose sum NIE + . . . is equal to the order of the extended covariance matrix, and the second partition is one integer parameter NIE. Except for these restrictions partitions are arbitrary and are determined by the user for his own convenience according to input and storage requirements.

The sample problem referenced in this description of UNCOVER input has an extended covariance matrix partitioned as illustrated in Fig. $I$ and has the integral experiment data as described in Table 1 . For this discussion, the extended covariance matrix is defined as a covariance matrix for all integral and differential experiments combined. Note that the sample problem includes data from eight integral experiments and that the extended covariance matrix is partitioned into $23 \times 23$ submatrices. The basic objective of UNCOVER input is the definition of the extended covariance matrix partitions and guidance of the code in accordance with these partitions in the assimilation of the associated sensitivity coefficient and covariance submatrices. 
XII

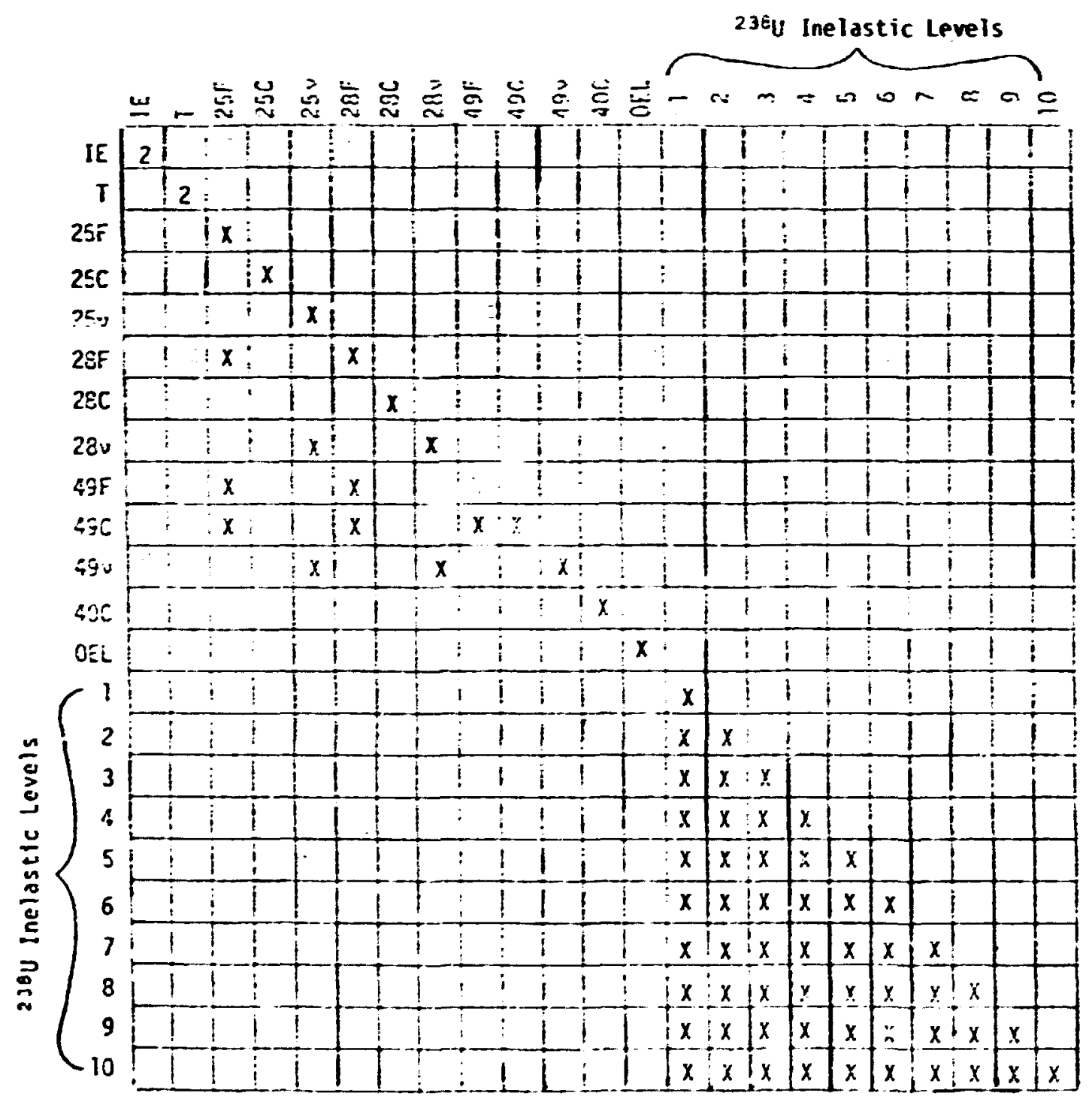

Fig. 1. Extended Covariance Matrix. 
Table 1. Integral Experiment Data Used for Adjustment ${ }^{a}$

\begin{tabular}{lccccc}
\hline Assembly & Response & Exp. & Calc. & $Y_{\text {Exp }}(\%)^{b}$ & Std. Dev. (q) \\
\hline ZPR-6/7 & $k$ & 1.000 & 0.9844 & 1.61 & 0.20 \\
& $28_{f} / 49 f$ & 0.02421 & 0.02344 & 3.32 & 1.78 \\
& ${ }^{28} \mathrm{c} / /^{49} f$ & 0.1406 & 0.1524 & -7.57 & 2.56 \\
ZPR-3/48 & $k$ & 1.000 & 0.9911 & 0.915 & 0.20 \\
& $k$ & 1.000 & 0.9885 & 1.18 & 0.15 \\
& $25 f / 49 f$ & 1.058 & 1.0140 & 4.37 & 1.32 \\
& $28 f / 49 f$ & 0.0300 & 0.0288 & 4.17 & 1.33 \\
& $28 c / 49 f$ & 0.1230 & 0.1311 & -5.96 & 1.46 \\
\hline
\end{tabular}

${ }^{a}$ This data was provided by ANL (R. McKnight and P. Collins).

${ }^{b} Y_{\text {Exp }}=(\operatorname{Exp}-($ Calc + Bias $)) / C a l c$ where bias designates known correc-

tions, e.g., homogeneous to heterogeneous, 1-D to 2-D, etc.

UNCOVER uses the container hlscik concept for data storage: all data is stored in a single block and base addresses are provided which point to the starting locations of subsets or arrays of daca within the container block. UNCOVER creates these base addresses and stores them in the 0\$\$ array.

The improved FIDO routine is used to input data into the various arrays of the container block. The features of this routine, as described in Chapter II permit considerable flexibility in the preparation of input data. The feature, which allows modification of data already in memory, and the capability to direct the acquisition of azta fiom external storage devices as well as from the card input stream are particularly useful.

The input of both the sensitivity matrix and the extended covariance matrix is by submatrix. The (NP-1)*NC submatrices of the sensitivity matrix 


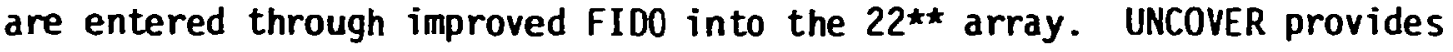
several al ternatives for entering the covariance submatrices. The first option (determined by IACSYM) is whether to enter all NPXNP submatric as of the symmetric covariance matrix or to apply symmetry and use the $N P(N P+1) / 2$ submatrices of the l:pper or lower triangle. Obviously, space is conserved by using the symetry. Another option (determined by MB) allows the (NPXNP or NP(NP+1)/2 according to the above option) submatrices of the extended covariance matrix to be stored all in memory or all on an external storage device (disk, tape, or drum): For these two alternatives the code calculates the base addresses and indicator of the $23 \$ \$$ array correctly.

If however (1) one wishes to mix storage of the covariance submatrices so that some are in core and some are on external storage devices, and/or if (2) one wishes not to store zero or unit subnatrices by the appropriate use of indicators in the 2355 array, and/or if (3) one wishes to make use of the fact that one submatrix is identical to another submatrix, then the addresses and indicators of the $23 \$ \$$ array must be entered by the user. Since in these circumstances the length of the core storage required in the $24^{\star \star}$ array for the extended covariance matrix is changed from that calculated by the code, the base address (in the 0\$\$ array) for the following $25 \$ \$$ array must be calculated and entered by the user. For these purposes the user should note that the base address for the $24^{\star \star}$ extended covariance storage array is always calculated correctly by the code and this base address is stored in two locations: (1) in the $25^{\prime}$ th word of the $0 \$ \$$ base address array and (2) the first word of the $23 \$ \$$ indicator array. The $Q$ and a input operations of improved FIDO can then be used to make the required address changes.

UNCOVER requires sensitivity coefficients and covariance matrices as input, and the SENPRO and COVERX files are the primary data bases for this information. However, rather than reading these files directly, UNCOVER reads compressed extracts from the files permitting more effective utilization of the improved FIDO input scheme. The COVERS module of the FORSS system reads SENPRO and COVERX files and prepares the extracts 
acceptable to UNCOVER. A description of the COVERS module is available in Chapter XIII.

UNCOVER INPUT

I\$S Integer parameters [10]

1. NP - Order of the first partition specifying the number of submatrices along a row (or column) of the extended covariance matrix

2. NC - Order of the second partition 1 for this versicn of the code

3. IACSYM - Options for defining extended covariance matrix $-1 \quad$ input only lower triangle submatrices

+1 input only upper triang?e submatrices

0 input all submatrices

4. ICLROS - Options for orderirig submatrices of the extended covariance matrix

+1 order submatrices by column

-1 orúer submatrices by row

5. MB - Storage options

2 all submatrices of extended covariance matrix to be in core

$>1000000$ all submatrices are in $1 / 0$ device $\mathrm{MB} / 1000000$

6. $M B B$ - Storage options

0 all submatrices of adjusted extended covariance matrix to be in core $>1000000$ submatrices are to be on the $1 / 0$ device MBB/ 1000000

7. LCOM - Edit option

$0 \quad$ Normal output

1 Hordes of intermediate result.s for trouble shooting 
8. IBCHK - Option to check $^{a}$ input covariance matrix B

0 No effect

1 Check is made

9. IBBCHK - Option to check ${ }^{a}$ output covärianrz matrix BB

0 No effect

$1 \quad$ Check is made

10. IESENS - Option to calculate from sensitivities

0 No effect

$>0$ Integral experiment adjustments are calculated

from sensitivities and cross section adjustments

$\mid \geq 2$ | Covariance of adjusted integral experiments

calculated from sensitivities and from the covariances of adjusted cross sections

$2 \$ \$$ First Partition Integer Parameters [NP]

1. NIE - The number of integral experiments.

2.

- The dimension associated with each type of differential

- experiment, typically the number of groups. In reference to

- a sensitivity partition, it is the length of the vector. In

- reference to the NP covariance matrix, it is the order of

- square matrix.

$3 \$ \$$ Second Partition Integer Parameters [NC]

NIE - The number of integral experiments.

T

20** Integral Experiment Data [NIE]

For each integral experiment enter:

Intecral experiment value - calrulated value Calculated Value

T

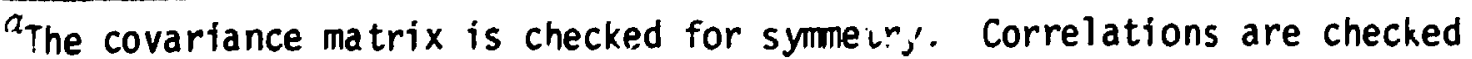
and a warning message is generated for each coirelation with an absolute magnitude greater than one.
} 
22** Relati:e Sensitivity Coefficients [SUM7*NIE], See COMMENTS ON THE $22^{\star \star}$ CARD for definition of SUMI.

T

0\$\$ Base Addresses [30]

Modify base address number 25 according te the memory required for covariance data. (Modify only if different from that calculated by the code).

24** Covariance Data

Relative Covariances of integral experiments and other relative covariance data which are not available on the external storage device(s) specified in the $23 \$ \$$ array.

$23 \$ \$$ Addresses and Indicators of the Extended Covariance

Matrix $\left[2{ }^{\star} \mathrm{NP} * \mathrm{NP}\right]$

IADDR (I), I=I, NP

$I N D(I), I=1$, NP (Enter only if the storage option provided by MB is to be overridden.)

Indicators are:

0 - Null submatrix

1 - Unit submatrix

-1 - Negative unit submatrix

2 - Slibmatrix stored in address specified

-2 - Transpcse of submatrix stored in address specified

$>10^{5}$ - Read subnatrix from unit and record designated and store in address specified

$<10^{\epsilon}$ - Read trans,ose from unit and record designated and store in address spe:ified

unit number $=\mid$ INO $\mid / 10^{6}$

record number $=\mid$ IND! - (unit number $\left.)^{\star}\right] 0^{\epsilon}$

T

27\$ NOMIT, (LIST(I), I=1, NOMIT)

!'MIT - The number of integral experiments to be omitted in adjustment. NOMIT is preset to 0.

LIST - A list of integral experiment number, which are to be omitted from the adjustment (assuming the order consistent with arrays 20,22, and 24). 
Note: If no integral experiments are to be omitted from the adjuctment, the $27 \$ \$$ card is not included in the input stream.

T

COMMENTS ON THE $22 * \star$ CARD

The first partition is made up of NP nuilbers, the first corresnonds to the number of integral experiments and the rest give the dimensicns of NP-1 types of differential experiments. A set of sensitivity coefficients or sensitivity submatrix is provided for each type of nuclear data, making NP-1 sensitivity submatrices. Each sensitivity submatrix contains sensitivity coefficients for every integral experiment response considered in the analysis. The sensitivity coefficients for a single response and for nuclear data of type I form a vector of length $L V(I)$, where $L V(I)$ is the $(I+1)$ th integer (on the $2 \$ \$$ card) which defines the first partition. The length of the I'th sensitivity submatrix for UNCOVER is LV(I) *NIE where NIE is the number of integral experiment responses. There are NP-1 such submatrices. The total number of sensitivity coefficients is NIE*SUMI is the sum of the $L V(I)$.

Note in the sample input the first card in the $22^{* *}$ array contains the fission spectrum temperature sensitivi uy coefficients for eight responses. This forms the first submatrix. (The fission spectrum temperature corresponds to a submatrix with dimension 1). The last three cards in the array direct that 21 submatrices be read from unit 31 , each submatrix firming one record. Note there are twenty-one 3162080 commands. The 31 is the $I / 0$ unit number, the $G$ is an improved FIDO read command, the 208 sensitivity coefficients reflect an LV(I) of 26 and an NIE of 8 , and the 0 indicates that unit 31 is unformatted (i.e., binary). The value of SUM1 is $547(=1+21 * 26)$. Unit 31 was prepared by the COVERS module which is discussed in Chapter XIII. 
The $0 \$ \$$ array contains the base address for the FIDO arrays used in UNCOVER. The code correctly calculates these base addresses under either of two a:sumptions: (1) all submatrices of the extended covariance matrix (hereafter referred to as the $B$ array) are assigned storage in the memory (the $24^{\star \star}$ array), or (2) all submatrices are stored on an 1/0 device and are read into the core (24** array) one submatrix at a time. If any submatrices are declared to be zero or if some are in core and some on an I/O device, then the length required for B-array core storage changes. This invalidates the precalculated $25 \$ \$$ array base address which in the $0 \$ \$$ array follows the $24^{\star \star}$ array base address. The user must insert the correct $25 \$ \$$ array base address via input into the $0 \$ \$$ array.

Note that the base address of the $24^{\star \star}$ array is stored in position 25 of the $0 \$ \$$ array, and that the base address of the $25 \$ 5$ array is stored in position 26 . First, the base address of the $24 * \star$ array is entered into position 26 of the $0 \$ \$$ array by FIDO command A26 1Q1. In the sample problem the correct $25 \$ \$$ array base address is determined by using the improved FIDO command A26 10741. This command causes the base address jusi put into position 26 to be incremented by 741 , which is the length required for B-array storage in the sample problem according to the partitioning and storage allocation used. For the sample problem, the length is determined as $N I E^{2}+1+I G M^{2}=741$, where the number of integral experiments NIE is 8 , and the number of groups IGM is 26 . NIE ${ }^{2}$ locations are reserved in memory for storage of the integral experiment covariances, one location is reservec in memory for the variance of the fission spectrum temperature. $I G M^{2}$ is the storage required to read from external storage the largest submatrix for the differential experiments. The operation of UNCOVER is such that it is unnecessary to correct any base address in the 0\$\$ array beyond position 26 . 
Accorsing to the partition chosen for our sample problem the extended covariance matrix is partitioned into $23 \times 23$ submatrices as shown in Fig. 1 . We have chosen to place two of these submatrices, denoted by 2 in Fig. 1 , into core storage. The first requires $8 \times 8 \quad(=64)$ locations and the second requires $1 \times 1(=1)$ location making a total of 65 locations required in core. The submatrices denoted by $X$ in Fig. 1 will be placed on an external device and will be read into core one at a time as needed. The largest such submatrix (in this example these externally stored submatrices are the same size) requires $26 \times 26$ (=676) locations. The remaining submatrices, denoted by blanks in Fig. 1 are all i.ero. Each will have an indicator 0 and will not be stored, and any bas address assigned to them will be ignored. The total memory requirec of the 2nk* array for storage and reading of the submatrices of the extinded covariance matrix is $741(=64+1+676)$.

Since the storage of the extended covariance matrix is mixed (some submatrices in internal memory and some on external storage devices) and also because not all submatrices are stored (some are simply indicated to be zero), UNCOVER cannot calculate the correct storage required for the $24^{\star \star}$ array. The user must input base addresses and indicators into the $23 \$ \$$ array and also must modify a base address in the $0 \$ \$$ array.

The 2355 array is made up of two sets of numbers, each set consisting of NPxNP numbers. The first NPXNP numbers of the 2355 array are the base addresses which specify where the sutmatrices of the extended covariance matrix $B$ are stored or into where they will be read. The second NFxNP numbers are indicators, one for each submatrix of the extended covariance matrix. The base address of the first submatrix of the $B$ matrix is always correctly calculated by UNCOVER and is the first number of the $23 \$ \$$ array.

Our strategy is as follows: after the first address (which already correctly gives the location of the first submatrix) we set all 528 remaining addresses to the address into which externally-stored submatrices will be read. Since the addresses of zero submatrices are ignored, we 
need to correct only one address, that for the internally stored fission spectrum variance, this address is one less than the address for reading in externally-stored submatrices.

Recall that the extended covariance matrix for the sample input is partitioned into $23 \times 23$ submatrices $(N P=23)$. He now define the base address for reading in the externally stored submatioices. The FIDO command A2 101 places the first address into the second location, to which the improved FIDO command 10 A2 65 adds 65 . (The addition of 65 provided space for the $8 \times 8$ integral experiment covariance matrix and the variance of the fission spectrum temperature.) The second address is now the correct base address for reading in the externally stored submatrices. The remaining 527 addresses are sct to this same address by the 0052701 command on the $23 \$ \$$ card. At this point, the base address for the temperature variance (submatrix number 25 ) is yet undefined. However, it is one less than the base address for reading the externally-stored submatrices. This is indicated on the $235 \$$ input cards by the improved FIDO command $10-1$. The following command of cancels the preceeding 10 comand. This completed the definitions of base addresses for the submatrices of the $B$ matrix.

The indicators for the subrnatrices of the B-matrix must now be entered into the 2355 array starting in location 530 (after the first $23 \times 23$ base addresses). The first submatrix, the covariances of the integral experiments, is stored in core - it was read by FIDO into the 24 * array. Hence the indicator for the first submatrix is 2 as shown on the second card for the 2355 array. The next 23 submatrices are zero matrices (see fig. 1) with 0 indicators. These are entered by the 232 following the 2 . The next submatrix is the temperature vis riance and was read by FIDO into the $24^{\star *}$ array and is in core storage. This is indicated by the next 2 , and following this 232 again indicates 0 covariances for the next 23 submatrices. 
At this point the next submatrix is stored externally, and all successive submatrices are stored externally or are zero. The module COVERS prepared these nonzero submatrices on unit 32 and also prepared the input stream as listed in Fig. 2.

\section{OUTPUT DISCUSSION}

Following the printing of the two correlation matrices, results are listed for each partition in the extended covariance matrix. Some of the output labels are self-explanatory. Perhaps the following will clarify others.

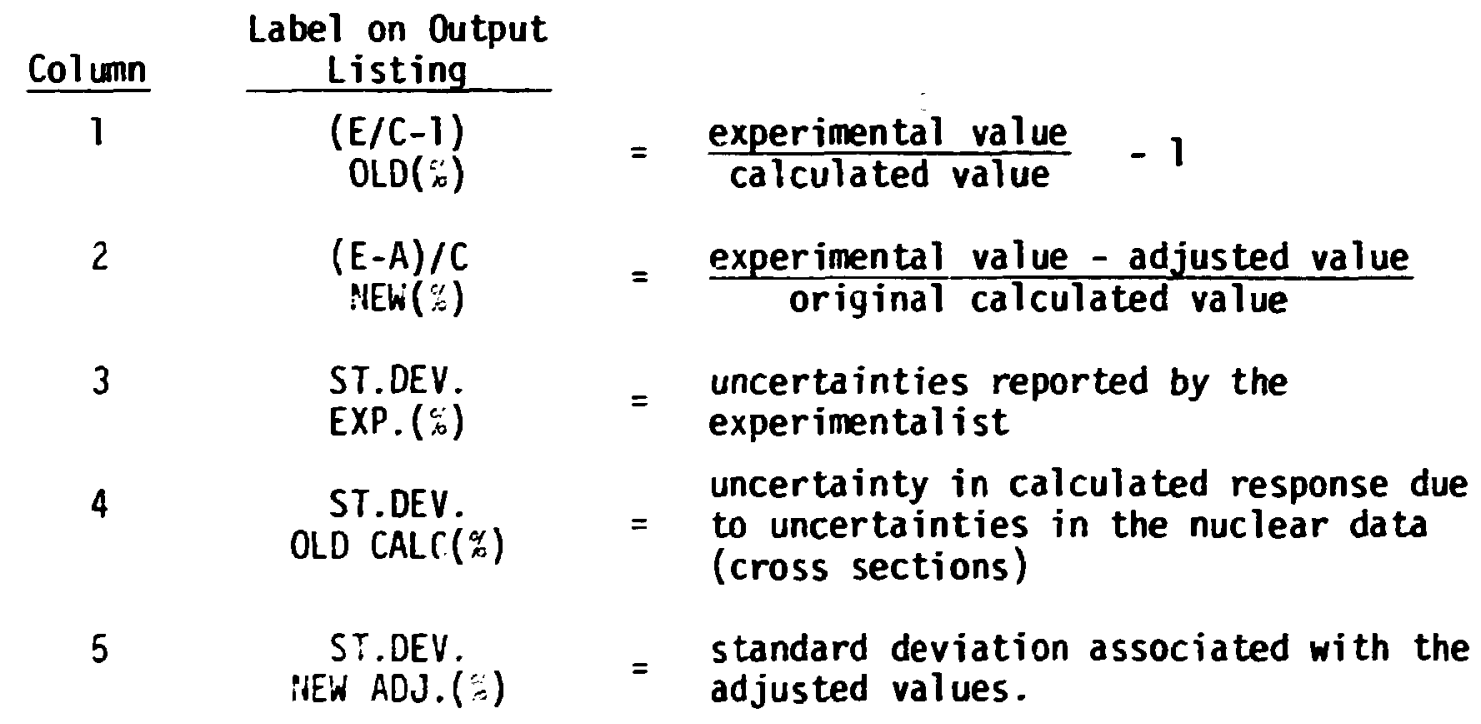

The results in output columns 6 - 9 are functions of the results in columns 1 - 5 as indicated below.

6

7

8

$$
\begin{aligned}
& \text { EXP. CHANGE } \\
& \text { (IN S.D.'S) } \\
& \text { CALC. CHANGE } \\
& \text { (IN S.D.'S) } \\
& \text { I } \\
& \text { S.D. RATIO } \\
& \text { (ADI./EXP) }
\end{aligned}
$$




\section{$X I I-17$}

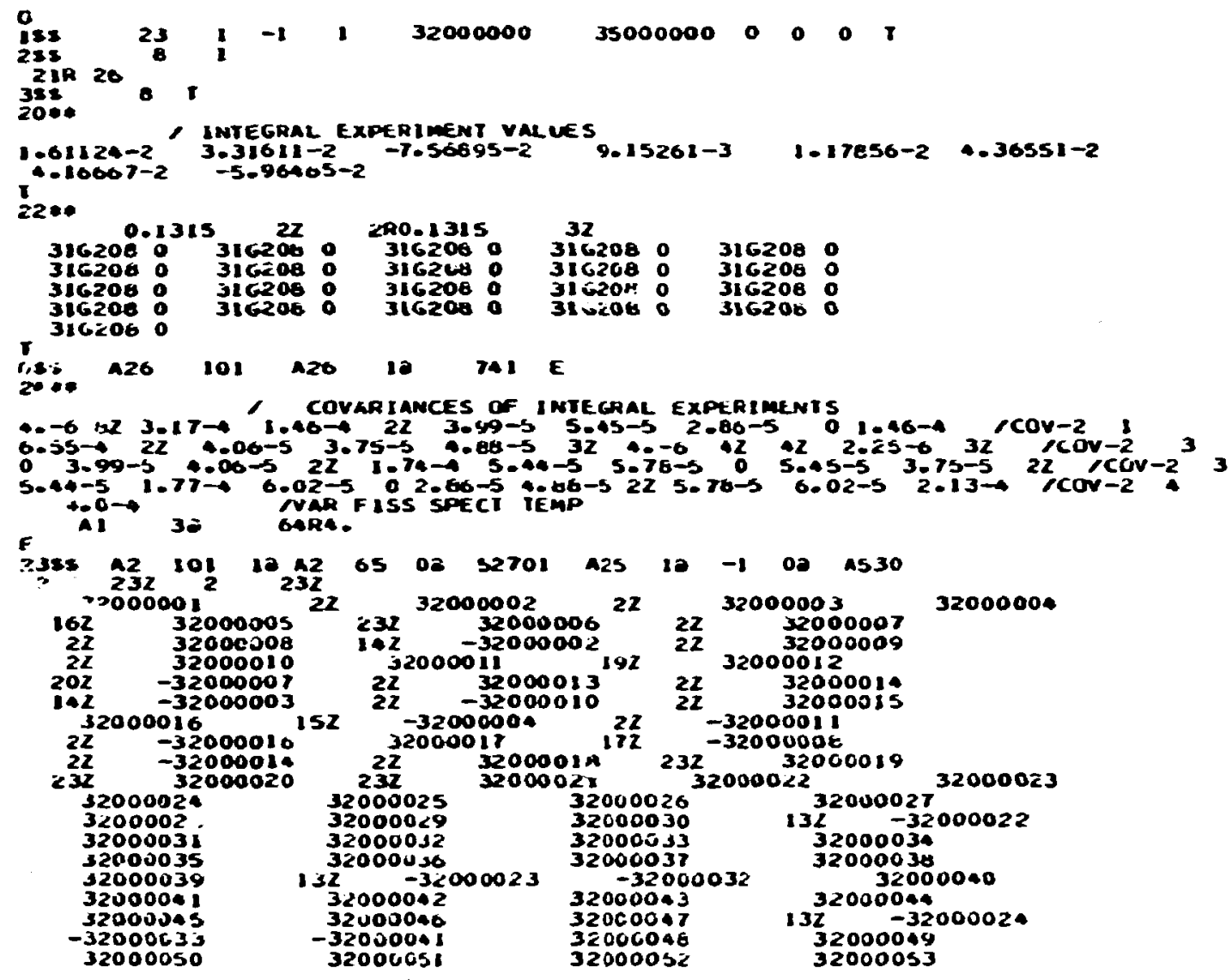

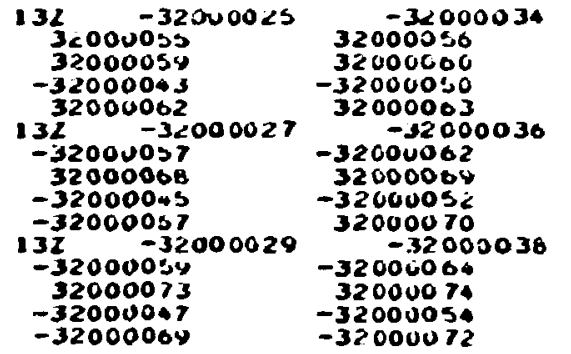

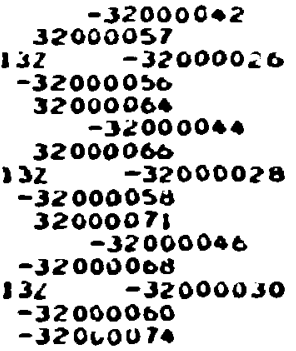

Fig. 2. List of Sample Irput 
Partition 2 ... NP - The Nuclear Data Partitions

1

2

3 $=\frac{\text { adjusted value }- \text { old value }}{\text { old value }} \times 100$

= standard deviation associated with nuclear data

$=$ standard deviation associated with adjusted nuclear data

The results in output columns 4 and 5 are functions of the results in columns 1, 2, and 3 as indicated below.

4

CHANGE

(IN S.0.'S)

$=1 / 2$

5

S.D. RATIO

(NEW/OLO)

$$
=3 / 2
$$

\section{UNCOVER ERROR STOPS}

STOP 1 - Problem size exceeds container array size. A printed message precedes the STOP 1 which specifies the memory required and the memory available. The container array size must be increased or the problem requirements reduced by partitioning.

STOP - Statement in subroutine COPYIT, see the printed message on the output.

STOF - Statement in subroutine COPYIT, see the printed message on the output.

STOP 13579 - The statement is located in subroutine UNPART. The numter of partitions in the extended covariance matrix exceeds 50 . A programing change is required unless the problem can be reduced. 
RESOURCE UTILIZATION

For the sample problem, the container block size was 20000 words, the program loaded and executed in $270 \mathrm{~K}$ bytes of core storage, execution time was 9 seconds on the IBM 360-91, $6851 / 0$ requests were made in the GO step, and four external storage units were used ( 1 for sensitivity coefficients, $l$ for covariance matrices, 1 for indicators, 1 for the adjusted extended covariance matrix). Not every problem requires external storage. 
CHAPTER XIII. THE COVERS MODULE OF THE FORSS SYSTEM
J. L. Lucius
J. H. Marable
J. D. Drischler 


\section{CHAPTER XIII}

Table of Contents

Page No.

I. INTRODUCTION ...................... XIII-3

II. INPUT PREPRATION FOR COVERS ............ XIII-3
A. The COVERS $1 \$ \$$ card ............... XIII-5
8. The COVERS $2 \$ \$$ Card ............ XIII-5
c. The COVERS $3 \$ \$$ Card ............ XIII-6

III. THE HOLLERITH DATA BLOCKS ............ XIII-10

A. Block 1, The Integral Experiment Data ...... XIII-10

B. Block 2, The Card Input Stream Sensitivity

Coefficients................ XIII-10

C. Block 3, The Card Input Stream Covariances.... XIII-11

IV. COVERS INPUT DATA DESCRIPTION ........... XIII-12

V. COVERS OUTPUT ......................... XIIIL

VI. COVERS ERROR STOPS .............. XIII-15

VII. COVERS RESOURCE UTILIZATION ........... XIII-15 


\section{INTRODUCTION}

COVERS is a convenience module which prepares input data for UNCOVER. The input requirements of UNCOVER which are described in Chapter XII should be reviewed before attempting to use COVERS. If all sensitivity and covariance data is to be made available to UNCOVER via the card input stream, then COVERS is unnecessary and the user should prepare input data directiy for UNCOVER. When all or part of the required sensitivity and covariance data is on a SENPRO and/or a COVERX file, COVERS can relieve some of the burden of input preparation. COVERS can prepare all of the input needed for an UNCOVER run. This consists of three data files: 1) an extract of the SENPRO file, 2) an extract of the COVERX fije, and 3) a file containing all of the card images required to define an UNCOVLR input case. However, there are cases when it is convenient to use the SEIPPRO and COVERX extract files, abandon the card image file, and prepare the card input stream directly in UNCCOVER. There are other cases when it is efficient to use the COVERS prepared input stream but modify it with card input directiy in UNCOVER.

\section{INPUT PREFARATION FOR COVERS}

Before attempting to prepare input for COVERS, it is suggested that an extended cuvariance matrix for the problem of interest be diagrammed similar to Fig. 1. This diagram illustrates the partitions in the extended covariance matrix, the cross correlations, and the source of data for each submatrix. Data as used in this context is to be interpreted as the sensitivity coefficients and covariances for each subratrix of the extended covariance matrix. Such data may be proyided via the 


\section{XIII -4}

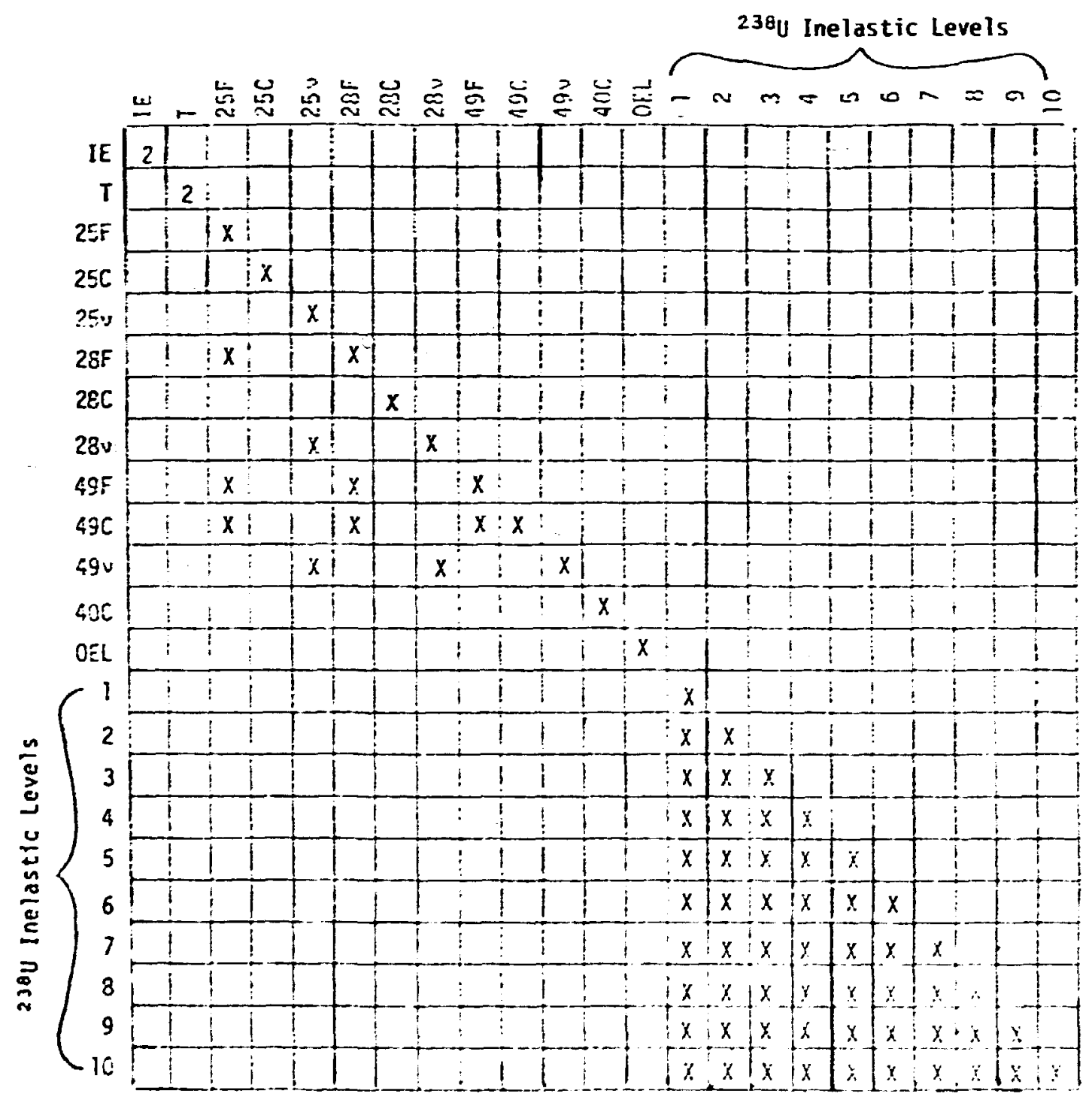

Fig. 1. Extended Covariance Matrix. 


\section{XIII-5}

the card input stream and/or obtained from SEN??O and COYERX files. Realistic problems iypically utilize data from both sources. COVERS input consists of Improved Fido Cards for the $1 \$ \div: 2 \$ \$$, and $3 \$ \$$ arrays which contain information used in COVERS computation and decision makir. COVERS also reads three other types of data in the Hollerith format. This information is passed to UNCNYER witnout modification and defines the UNCOVER $20^{\star \star}, 22^{\star \star}$, and $24^{\star *}$ input arrays.

\section{f. The COVERS $1 \$ \$$ Card}

This card contains administrative infonation, and the brief definitions of Each parameter i.. the COVERS Innst Data Description section sho'sld suffice with the exception of the ninth parameter, KRECT. KRECT is a modifier for input data contained on the $3 \$ \$$ cards and its use will? be described in the discussion of the $3 \$ \$$ cards. KRECT is a very useful input parameter, but it is aiso very dangerous. if iinproperly used, disaster is certain. The nost prudent use of KRECT is to initially set its value to zero and le ve $i$ : unchanged until the need for a change is clearly demon' wited and cinderstood.

\section{B. The COVERS $2 \$ \$$ Card}

The irifor nation on this card is a list of the experiments included in the adju,tiient. SENPRO nomenclature is used; i.e., assembly identifier and response identifiers isee the discussion of the SENPRO file MAT-MT control recorú in Appendix. A of Chapter VIII). This information, along with the matorial-reaction type identifiers provided on the $3 \$ \$$ cards, identifies all of the sensivivity profiles located on a SENPRO file to be used in the arjustnent. 


\section{The COVERS $3 \$ \$$ cards}

The $3 \$ \$$ cards provide a transcription of the diayram in Fig. 1 of the s.xtended covariance matrix into functiolis? numerical nomenclature. The $3 \$ \$$ cards serve the following functions:

1. Delineate the partitions included in the extended covariance matrix.

2. Indicate the source of data for each SL'bmatrix of the extended covariance matrix. Recail that as used in this context, data is to be interpreted as sersitivity profiles and covariances. Two sources are available, the card input stream and the files (SENPRO, COVERX).

3. Identify the cross correlations needed between material-reaction type pairs. The correlation of a material-reaction type to itself is assumed.

4. Coupled wich the $2 \$ \$$ card, identify all sensitivity profiles to be used in the adjustment.

5. Identify all of the covariances needed in the adjustment.

In discussir.g preparation of the $3 \$ \$$ cards, ieference will be made to the sample input showr in Fig. 2. COVERS permits only the lower triangle of the extended covariance matrix to be described and then guarantees symmetry. The content of a $35 \$$ card is different depending upun whether the data is to come from the card input stream or from the files (SENPRO, COVERX). CCVERS requires that all partitions requiring data from the card input stream be described before the partitions obtaining data from the files. If the sensitivity data is provided via the card input stream so must be the covariance data and converse,y. Refer 


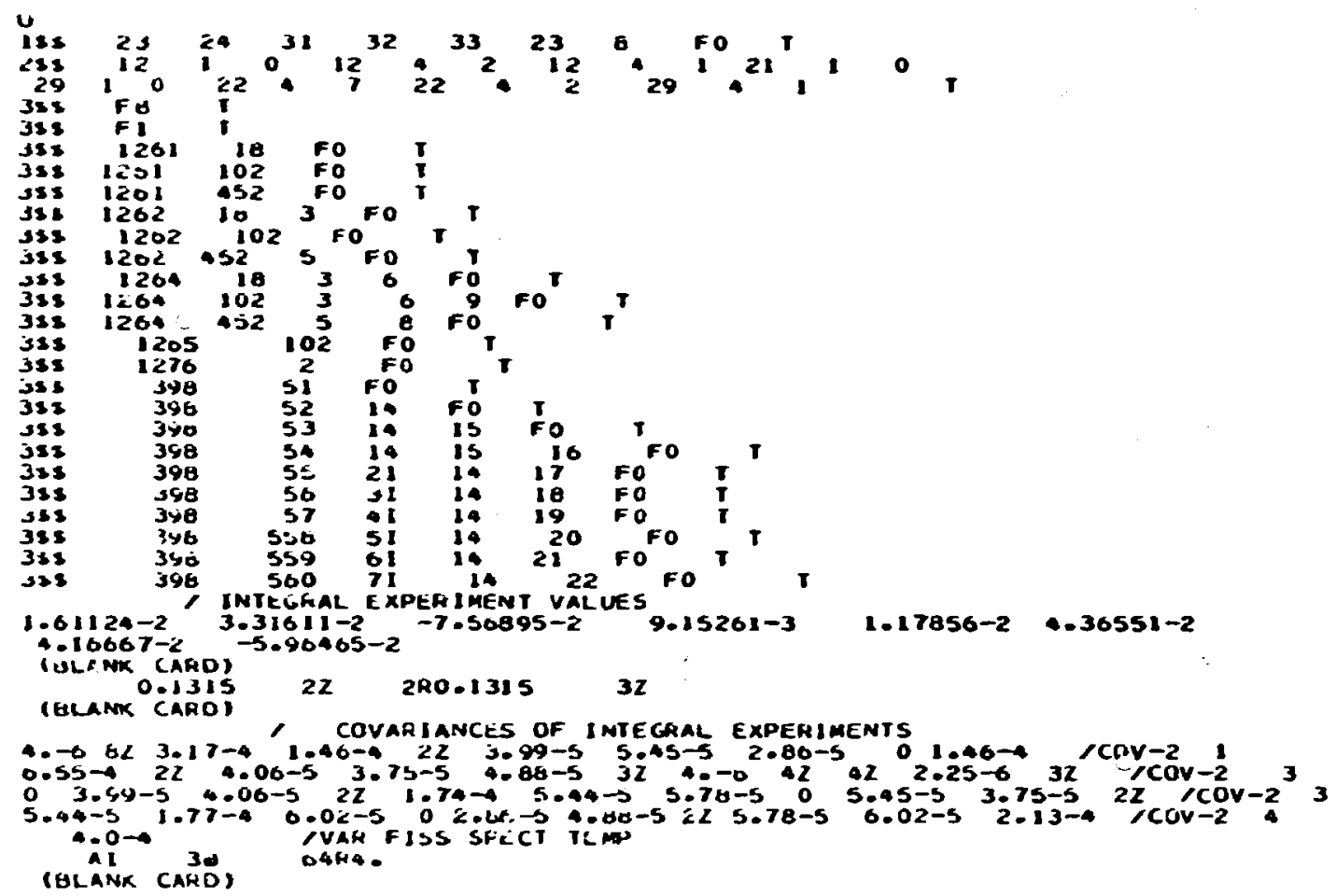

Fig. 2. Sample Iriput for a COVERS Case.

to the diagram of the extended covariance matrix in Fig. 1; in reading down the diagonal from left, to right, notice the two integers with a value of 2. These indicate that the data for these submatrices will be provided in the card input stream immediately following the $3 \$ 5$ cards. The corresponding $3 \$ 5$ cards contain information of a dimensional nature; i.e., how many numbers are to be read from cards. Note in Fig. 2, the content of the first $3 \$ \$$ card is $3 \$ \$ F 8 T$. This card deals with the integral experiment partition which is unique in that no sensitivity coefficients are entered for the partition. UNCOVER generates the sensitivity coefficients for the integral experiment partition internally. However a covariance matrix must be input for the integral experiment sartition and the 35 S 8 T card directs that 64 numbers, an $8 \times 8$ covariance matrix, be entered in the covariance block. In Fig. 2 , the 


\section{$X I I I-8$}

second 355 card content is $3 \$ \$ F 1 T$. This partition deals with the temperature associated with a fission spectrum. The first eight rumbers in the sensitivity block of the card input are the sensitivity to the temperature of each of the eight integral experiments and the 65th entry in the covariance block is the variance of the temperature. Recall the first 64 words of the covariance block have been used for the $8 \times 8$ covariance matrix associated with the integral experimert partition. Data ior the integral experiment partition must be entered first and immediately followed by all of the other partitions for which the data is entered from the card input stream. The order in which sensitivity and covariance data is entered in their respective blocks cstablishes the correspondence.

Now consider the content of the $3 \$ \$$ cards that deal with the submatrices for which data is obtained from the files (SENPRO, COVERX). The objective is to identify all of the sensitivity profiles and all of the covariance matrices needed in the adjustment and to read this data from a SENPRO file and a COVERX file. Recall that a sensitivity profile on a SENPR.O file has five identification numbers: 1) assembly, 2) response, 3) material, 4) reaction tyci, and 5) reaction rate ratio. Obviously items 2 and 5 are related and merely constitute a dual identification of a response. Identificition of covariances is simpler. Covariance matrices are not assembly and response dependent. They are identified by four integers material-reaction type correlated to the same niaterial-ruaction type or cross correlated to another material-reaction type. Note that the third partition of the extended covariance matrix in Fig. 1 indicates a correlation of $25 \mathrm{~F}$ to itself. In Fig. 2, note 


\section{XIII-9}

that the third 355 card is comp?eted as $3 \$ \$ 126118$ FO T. The 1261 is the riaterial identifier for ${ }^{235} U$ on both the SENPRO and COVERX files and the 18 is the reaction type identifier for fission on both files. The actual numerical values of these identifiers are arbitrary, but they niust be consistent on the $3 \$ \$$ cards, the SENPRO file, and the COVERX file. The ENDF/B material-reaction type (MAT-MT) identifiers serve very effectively as identifiers in the adjustment process. With the assemblies and responses idenzified on the $2 \$ 5$ cards and the material-reaction type identified on the $3 \$ \$$ cards, COVERS can search a SENPRO file for the sensitivity profiles for all assemblies and all responses to ${ }^{235} \mathrm{U}$ fission. Also, COVERS can search a COVERX file for the covariance of ${ }^{235} \mathrm{U}$ fission correlated to itseif. This process is now repeated for every partition of the extended covariance matrix. io further illustrate the preparation of $3 \$ \$$ cards, consider the sixth partition of the extended covariance matrix. It is indicated that $28 \mathrm{~F}$ is correlated to itself and cross correlated to $25 \mathrm{~F}$. Referring to the sixth $3 \$ \$$ card in Fig. 2 , the 1262 is the MAT number for ${ }^{238} \mathrm{U}$ and 18 is the MT number for fission. The 3 indicates the cross correlation to $25 \mathrm{~F}$ since $25 \mathrm{~F}$ has already been identified in the third partition of the extended covariance matrix, hence the 3 on the $3 \$ \$$ card. It may also be convenient to note in fig. I that the $x$ indicating the cross correlation is in colimn 3 of the extended covariance matrix. Now suppose all input is prepared and it is decided that one additional partition with data in the card input stream should be added. Recall all card input particiolis must precede the partitions with data from the files. The 3 in partition 6 used to indicate the cross correlation of $28 \mathrm{~F}$ to $25 \mathrm{~F}$ now should be 4 and every other cross 
correlation indicator shouid be incremented by one. Before discarding all input and stacting over, examine the $15 \$$ card, item 9 , the parameter KRECT. When KRECT is nonzero, all cross correlation indicators are redefined as $K N^{-}=K N+K R E C T$. Where $K N$ is the input value but $K^{-}$is the value actually used by the code to establish cruss correlations.

\section{THE HOLLERITH DATA BLOCKS}

COVERS reads three Hollerith data blocks and passes the data directly to the UNCOVER card input stream to form the UNCOVIR input arrays designated as $20^{\star \star}, 22^{\star \star}$, and $24^{\star \star}$. COVERS does not have $\exists$ need to know any numerical values of this data and treats all of these cards as Hollerith information. However, the user must know how much and what information to prepare. A review of the UNCOVER input requirements in Chapter XII would be helpful, but all of the "how much" question is already answered in the preparation of the COVERS 1\$5, 255, and 355 cards.

\section{A. Block 1, The Integral Experiment Data}

Enter one value (V) for each integral experiment: $V=\frac{V M-V C}{V C}$, where $V M$ is the measured value of the experiment and $V C$ is the calculated value of the experiment. Data must be entered in this block. End the block with a blank card.

B. Block 2, The Card Input Stream Sensitivity Coefficients

Excluding the 355 card for the integral experiment partition, examine all of the $3 \$ \$$ cards ielevant to partitions with sensitivity and covariance data being entered via the card input stream. In the 
sample input shown in Fig. 2, this is one card $35 \$ \mathrm{Fl}$ T. From the seventh parameter on the $15 \$$ card it is known that the problem includes eight integral experiments; therefore, $1 \times 8$ is the number of sensitivities to be entered in Block 2. This completes the Block 2 data entry for the sample input, but assume following the $3 \$ \$$ F1 $T$ card there was another $3 \$ \$$ card with the content $3 \$ \$ \mathrm{~F} 4 \mathrm{I}$. In this hypothetical case, the total number of sensitivities to be entered would be calculated as $(1+4) \times 8$. The first eight entries correspond to the 355 card (3\$\$ F1 T) and the following 32 entries would correspond to the hypothetical $3 \$ \$$ card ( $3 \$ \$$ F4 T). The 32 entries must be defined in the following order. four sensitivities for the first integral experiment, four sensitivitias for the second integral experiment, etc, until four sensitivities have been entered for each of the eight integral experiments. If all sensitivity and covariance data were to be obtained from the files (SENPRO, COVERX), Block 2 would be empty. Orie blank ca.d is always used to end Block 2, designating either the empty condition or the end of card input stream sensitivity data.

\section{Block 3, The Card Input Stream Covariances}

A square covariance matrix must be entered for each partition for which the card input stream is the source of data. In Fig. 2, note the first two $35 \$$ cards: $3 \$ \$ F 8 T, 3 \$ \$ F 1 T$. The number of values to be entered in Block 3 is calcuiated as $8^{2}+1^{2}$. If all sensitivity and covariance dato were to be obtained from the files (SENPRO, COVERX), Block 3 would be empty. One blank card is always used to end Block 3 , designating either the empty condition or the end of card input stream covariance data. 


\section{XIII-12}

\section{COVERS INPUT DATA description}

$1 \$ \$$ Inteyer parameters (12)

1. IPRO - unit numer of SENPRO file

2. Icos - unit number of COVERX tile

3. JAMS - unit number of data extracted from SENRPO

4. JAMC - unit number of data extracted from COVERX

5. JAMI - unit number of UNCOVER card image input stream

6. NP

- number of partitions in extended covariance matrix

7. NRESP - number of responses (integral experiments)

8. LCOM - edit option

0 - normal output

$>0$ - full output for troubleshooting

9. KRECT - constant to be added to KN, see 355 card,

KRECT may be negative

10. IBCHK - Option to check $^{a}$ input covariance matrix 8

0 - no check

1 - check

11. IBBCHK - option to check $^{a}$ output covariance matrix BB

0 - no check

1 - check

12. IESENS - option to calculate from sensitivities

$$
0 \text { - no effect }
$$

${ }^{2}$ The ccvariance matrix is checked for symmetry. Correlations are checked and a warning message is generated for each correlation with an absolute magnitude greater than one. These checks have the potential for generating thossands of lines of print. 


\section{XIII-13}

$$
\begin{aligned}
& >0 \text { - integrai experiment adjustments calculated } \\
& \text { from sensitivities and cross section adjustments } \\
& |\geq 2| \text { - covariance of adjusted integral experiments } \\
& \text { calculated from sensitivities and from the } \\
& \text { covariances of adjusted cross sections }
\end{aligned}
$$

T

2\$\$ Integer parameters (3*NRESP) response description

$$
\begin{aligned}
& \text { IASB - assembly identification } \\
& \text { IRESP - response identification } \\
& \text { NITR - reaction rate ratio identification }
\end{aligned}
$$

complete for all responses

\section{T}

The $3 \$ \$$ card indicating data is to come from the card input stream and has the following form:

$3 \$ \$$ Integer parameters $(100)$

F ND T

The array is filled w:th the dimension (ND) associated with the experiment being described. On the first $3 \$ \$$ card, ND is equal to the number of integral experiments. On succeeding $3 \$ \$$ cards, the associated dimension is typically the number of groups. In reference to a sensitivity partition, it is the length of the vector. In reference to a covariance matrix, it is the order of the square matrix. 


\section{XIII-14}

The $3 \$ \$$ card indicating data is to come from SENRRO and COVERX files and has the following form:

$3 \$ \$$ Integer parameters (100)

MAT - material identification jor the row

$M T$ - reaction type identification for the row

KN - the partition column numbers of all nonzero covariance submatrices in the row and to the ?eft of the diagonal element

FO - the FIDO fill with 0 option; this input is mandatory

$T$

The following three types of data are card images which COVERS uses to prepare the $20^{\star \star}, 22^{\star \star}$, and $24^{\star \star}$ arrays for UNCOVER. Note each type of data is terminated with a blank card.

Block 1, Integral experiment data (NIE)

For each integral experiment enter:

\section{Integral experiment value - calculated value} calculated value

End with a blank card.

Block 2, Sensitivit, coefficients

Enter sensitivity coefficients for each differential experiment not retrieving coefficients from a SENRPO file. End with a blank card. Block 3, Covariance data

Enter the covariances of integral experiments and other covariance data which are not available on a COVERX file. End with a blank card.

\section{COVERS OUTPUT}

If a sensitivity profile is needed for the problem and cannot be located on the SENPRO file, COVERS indicates the profile was not found 


\section{XIII-15}

and proceeds as if the profile was found with all coefficients for that profile set to zero. COVERS also indicates what covariance matrices are required and those that are found. Any not found are set to zero.

Three files are prepared by COVERS: an extract of SENRPO, an extract of COVERX, and the card stream input for UNCOVER.

\section{COVERS ERROR STOPS}

STOP 1 at least one $3 \$ \$$ card did not end with 0 , check $3 \$ \$$ cards STOP 2 all $3 \$ \$$ cards were filled with 0 , COVERS has nothing to do, check $3 \$ \$$ cards

STOP 3 ) the container array is too small to accommodate STOP 4$\}$ the problem, reduce problem or increase the size STOP $5 \int$ of the container array

VII. COVERS RESOURCE UTILIZATION!

For the sample problem the container block size was 20,000 words, the problem loaded and executed in $180 \mathrm{~K}$ bytes of core storage, execution time was five seconds on the IBM $360-91,3861 / 0$ requests were made in the GO step. Two external storage units were required for the SENRPO and COVERX files, three external storage units were used for the SENRPO extracts, the COVERX extracts, and the UNCOVER card input stream. 


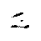 \\ CHAPTER XIV. THE ADJUST MODULE OF THE FORSS SYSTEM \\ J. L. Lucius \\ N. M. Greene \\ J. J. Wagschal
}


CHAPTER XIV

Table of Contents

Page No.

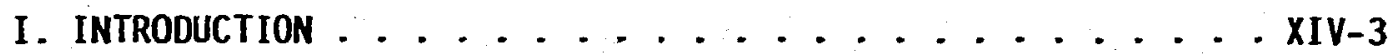

A. Correspondence Between UNCOVER and the AMPX Master File XIV-3

B. Adjustment of Fission Spectrum Parameters ....... XIV-3

C. The ADJUST $2 \$ \$$ and $3 \$ \$$ Input Arrays ......... XIV-5

D. Preparation of a Consistent Cross Section Set with AMPX-COMET .................. XIV-5

II. ADJUST INPUT $\ldots \ldots \ldots \ldots \ldots \ldots \ldots \ldots$

REFERENCES ........................... XIV

APPENDIX A. Fission Spectrum Parameter's......... XIV-13

APPENDIX B. AJAX -- AMPX Module to Merge, Collect, Assemble, Re-Order, Join and/or Copy Selected Data Sets from AMPX

Master Interfaces .................. XIV-15 


\section{THE ADJUST MODULE}

\section{INTRODUCTION}

The preparation of a functional, adjusted cross section set is a three step operation involving the UNCOVER module, the ADJUST module and the AMPX ${ }^{1}$ modules, COMET, AJAX, DIAL AHI RADE. The objective is to modify (adjust) an AMPX multigroup, infinitely dilute, master cross section file with the changes calculated by an UNCOVER adjustment run resulting in an adjusted cross section file in the AMPX master format. Extreme care must be exercised to insure that a consistent cross section set is produced. This involves application of the AHPX module COMET in a manner consistent with the type of adjustment made.

\section{A. Correspondence Between UNCOVER and the AMPX Master File}

A correspondence is established between each UNCOVER different ial parameter (e.g., cross section) partition, and one or more materialreaction types on an AMPX master file. Tne multiple currespondence is especially useful in the adjustment of inelastic levels. UNCOVER produces: a file containing one record for ea n differential partition in the UNCOVER run. Typically, each record contains the percent changes by energy groud to be used in adjusting the corresponding material-reaction type(s) on the AMPX master. The AMPX master and the UNCOVER results must be in either identical group structures or the UNCOVER structure must be a subset of the group structure on the AMPX master. With bcth group structures available, ADJUST applies the UNCOVER percent changes over the appropriate energy ranges of the AMPX inaster.

\section{B. Adjustment of Fission Spectrum Parameters}

Unlike other reactions, the fission spectrum on the AMPX master is not mechanially adjusted by a multiplicative constant facior, but is rather replaced by recalculating group values based on modified parameters. UNCOVER produces percent changes to be applied to these parameters, wich are passed to ADJUST wich calculates a new fission spect.rum and places it on the AMPX master. Both the Watt ${ }^{2}$ and Maxwellian ${ }^{2}$ definitions of a 
fission spectrum are available (although ENDF/B-V allows for energy dependent parameters $a$ and $b$, since in the AMPX master file there is only one fission spectrum, cnly one set of parameters ver material is handled):

\section{The Maxwellian fission spectrum}

$f\left(E+E^{\prime}\right)=\frac{\sqrt{E^{\prime}}}{I} e^{-E^{\prime} / a}$

$I$ is the normalizatien constant,

$I=a^{3 / 2}\left[\frac{\sqrt{\pi}}{2} \operatorname{erf}(\sqrt{(E-U) / a})-\sqrt{(E-U) i^{\prime} a} e^{-(E-U) / a}\right]$

\& ic th- „djusted parameter input from a $4^{k \star}$ card.

$U$ is a constant introduced to define the pioper upper limit for the final neutron energy such that $0 \leq E^{\prime} \leq E-U$.

The Hatt fission spectrum

$f\left(E+E^{\prime}\right)=\frac{e^{-E^{\prime} / a}}{I} \sinh \left(b E^{\prime}\right)$

I is the normalization constant,

$$
\begin{aligned}
I=\frac{1}{2} \sqrt{\frac{\pi a^{3} b}{4}} \exp \left(\frac{a b}{4}\right)\left[\operatorname{erf}\left(\sqrt{\frac{E-U}{a}}-\sqrt{\frac{a b}{4}}\right)+\operatorname{erf}\left(\sqrt{\frac{E-U}{a}}+\sqrt{\frac{a b}{4}}\right)\right] \\
-a \exp \left(-\left(\frac{E-U}{a}\right) \sinh (\sqrt{b(E-U)})\right)
\end{aligned}
$$

$a$ and $b$ are adjusted parameters input from a $4^{\star *}$ card

" is a constant introduced to define the proper upper for the final neutron energy such that $0 \leq E^{\prime} \leq E-U$

To calculate a Watc Spectrum, the user must determine the values of the constants $a$ and $b$ at i MEV wich were used to calculate the corresponding Watt spectrum on the AMPX master (see AppendfX A). The UNCOVER results (percent change of $a$ and $b$ ) must then be applied by hand 
to calculate the adjusted values of $a$ and $b$ and be input to ADJUST (4*t cards). The same procedure applies for a Maxwellian spectrum with the constant ainterpreted as the Maxwellian $\theta$ and the constant $b$ set to zero. The hand operations are required because values for $a, b$, and $\theta$ are currently not available on the AMPX master and the retrieval of these parameters from a basic ENDF/B-V file is beyond the scope of this version of P.DUST.

\section{The ADJUST $2 \$ \$$ and $3 \$ \$$ Input Arrays}

The ADJUST input to implement the correspondence tetween UNCOVER and the AMX master file is provided in the $2 \$ \$$ array and the $3 \$ \$$ arrays. Typically there is a one-to-one correspondence between an UNCOVER partition and a $3 \$ \$$ array. The correspondence is established by inspecting each UNCOVER partition and determining the applicable corresponding AMPX master material (MAT) and reaction type (MT) identifiers which are then entered in $3 \$ \$$ arrays $i$ - te same order. A single UNCOVER partition may be designed to adjust parameters for more than one material-reaction type. For example, the parameters $a$ and $b$ may be adjusted for both $239 \mathrm{pu}$ and $240 \mathrm{pu}$ in the same UNCOVER partition. In this situation, a $3 \$ \$$ array must be prepared to identify each material treated in the partition. The $2 \$ \$$ array contains an indicator for each 3\$; array in the input stream. The indicator defines the relationship of the material-reaction type(s) specified in the $3 \$ \$$ array with the corresponding UNCOVER partition. Also, a specific adjustment may be desired that does not require the results of every pirtition included in UNCOVER run. This condition can be indicated in the $2 \$ \$$ array.

\section{Preparation of a Consistent Cross Section Set With AMPX-COMET}

Before an adjusted AMPX master can be used in calculations, AMPX-COMET must be used to calculate the following:

1. The neutron disappearance cross section $(M T=101)$ is the sum of all partial disappearance cross sections (i.e. $(n, r),(n, p)$, $(n, \alpha), \ldots)(M T=100+i, 1=2, \ldots, 14)$ 
2. The absorption cross section $(M T=27)$ is the sum of the disappearance ( $\mu T=101)$ and fission $(M T=18)$ cross sections.

3. The total cross section as a sum of partials

4. For materials were the inelastic total cross section has been adjusted, the inelastic levei cross sections must be normalized to sum to the adjusted inelastic total. For materials where the inelastic level cross sections have been adjusted, the inelastic total must be calculated as a sum of the adjusted levels.

AFPX-RADE may used to check the internal consistency of the cross section set. AMPX-AJAX is very useful in isolating materials requiring the specific treatments discussed in step 4 and then recombining all of the materials onto a single AMPX master. AMPX-DIAL may be used to edit the sross sections residing on an AMPX master file.

\section{ADJUST INPUT}

$1 \$ \$$ Integer Parameters [6]

1. NR - The number of differential parameter sets in the UNCOVER case. NR is also the number of $3 \$ \$$ cards to be prepared.

2. HOMT - The maximum number of reaction types to be adjusted by the same factor. NOMT will be equal to the maximum number of reaction types identified on a single $3 \$ \$$ card (default $=$ 1), MOMT $>1$ is useful for adjusting inelastic levels when several levels are to receive the same adjustment.

3. N61 - The unit number of the file containing the UNCOVER results. (percent changes) (default $=61$ ).

4. MMG - The unit number of the AMPX master file to be adjusted (default $=45)$.

5. NAD - The unit number of the adjusted AMPX master file (defarlt = 46).

6. ISU - The unit number of a SENPRO file containing the group boundaries and sensitivity coefficients used in the UNCOVER run (default $=23$ ). 
2\$\$ Integer Parameters [NR]

These parameters describing the requested operation for each $35 \$$ card assume one of the following values for each $3 \$ \$$ card.

-1 - This indicates a fission spectrum is to be calculated from the adjusted parameters $a$ and $b$ which will be input on a $4^{\star \star}$ card. A record on unit $N 61$ is read but the contents are discarded.

-25 - This indicates the same action as -1 above with one important exception; no corresponding record exists on unit $N 61$ and no attempt is made to read such a record.

0 - This indicates that a record exists on unit $N 61$ but that the information is not useful in this particular adjustment. The record is read and the contents iscarded, i.e., the recr skipped.

+1 - This indicates a record is read from unit $N 61$ and the con is are used in the adjustment.

$T$

Prepare a $3 \$ \$$ card $^{\dagger}$ for each differential experiment (partition) in the UNCOVER run, i.e., prepare NR (see $1 \$ \$$ card) $3 \$ \$$ cards

$3 \$$ Integer Parameters [NOMT + 1]

MAT - The material identifiers on the AMPX master of int material to be adjusted by the percent changes calculated in the ccresponding UNCOVER partition.

MT - The reaction type identifier on the AMPX master of the reaction to be adjusted by the percent changes calculated in the corresponding UNCOVER partition. A maximum of NOMT (see 15\$ card) reaction types may be adjusted by the same percent changes. This is useful for inelastic levels.

F 0

$T$

fFor records to be skipped, the $3 \$ \$$ card should be filled with zeros (3\$\$ FO T). 
$X I V-8$

4** Adjusted Fission Spectrum Parameters [2]

a - This parameter is interpreted as the adjusted value of the constant $a$ used in the definition of a Hatt Spectrum. For a Maxwellian spectrum, this parameter is interpreted as the constant $\theta$.

$b$ - For a Hatt spectrum, this parameter is the adjusted value of the constant $b$. Enter a value of zero for this parameter to calculate a Maxwellian spectrum.

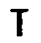

Enter one $4^{* *}$ card for each fission spectrum identified by a $3 \$ 5$ card in the same order as the $3 \$ \$$ fission spectrum identifiers are encountered.

AMPX Input

Relevant sections of reference 1 have been reproduced and inserted in APPENDIX B for convenience.

\section{REFERENCES}

1. N. M. Greene, J. L. Lucius, L. M. Petrie, H. E. Ford III, J. E. White R. G. Hright, "AMPX: A Modular Code System for Generating Coupled Multigroup Neutron-Gamma Libraries from ENDF/B," OPNL, 'MM-3706 (March 1976). See also RSIC PSR-63/AMPX-II Code Package Material (1978).

2. R. Kinsey, "Data Formats and Procedures for the Evaluated Nuclear Data File, ENDF," BNL-NCS-50496 (ENDF 10:2), 2nd Edition (ENDF/B-y) (1979). 
Figure 1: Sample Input for ADJUST and AMPX (AJAX, COMET, RADE)

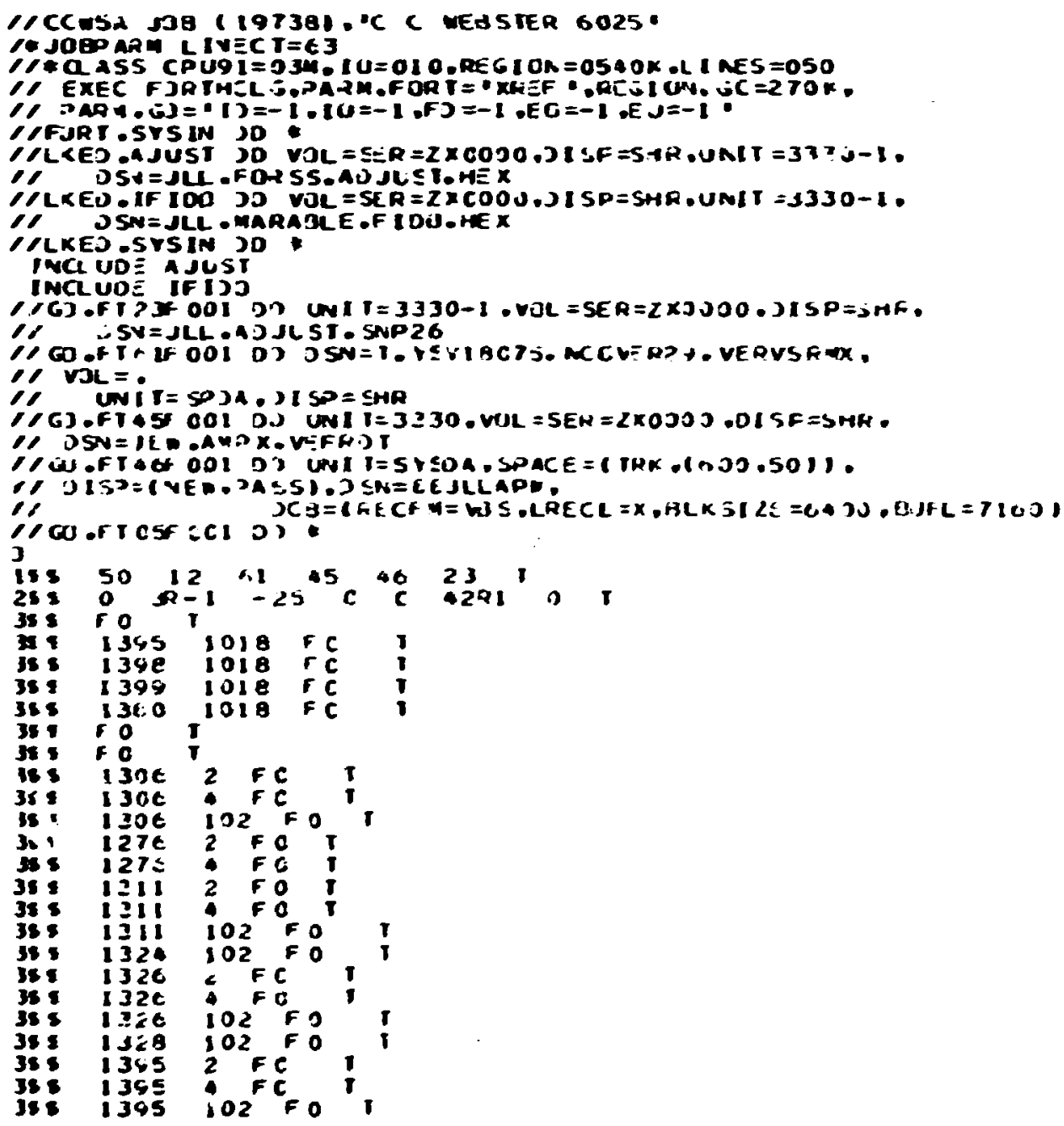




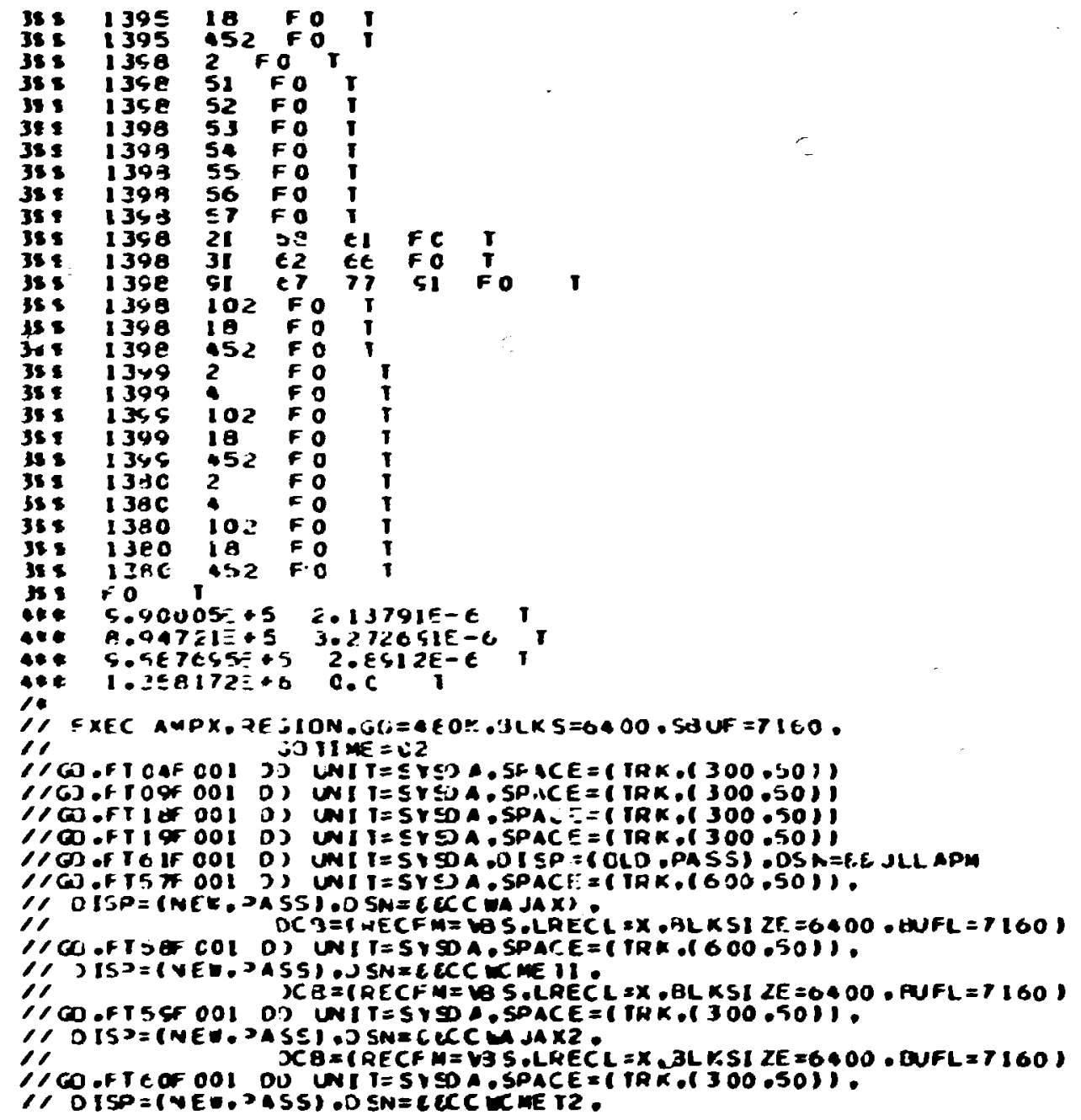




\section{XIV-11}

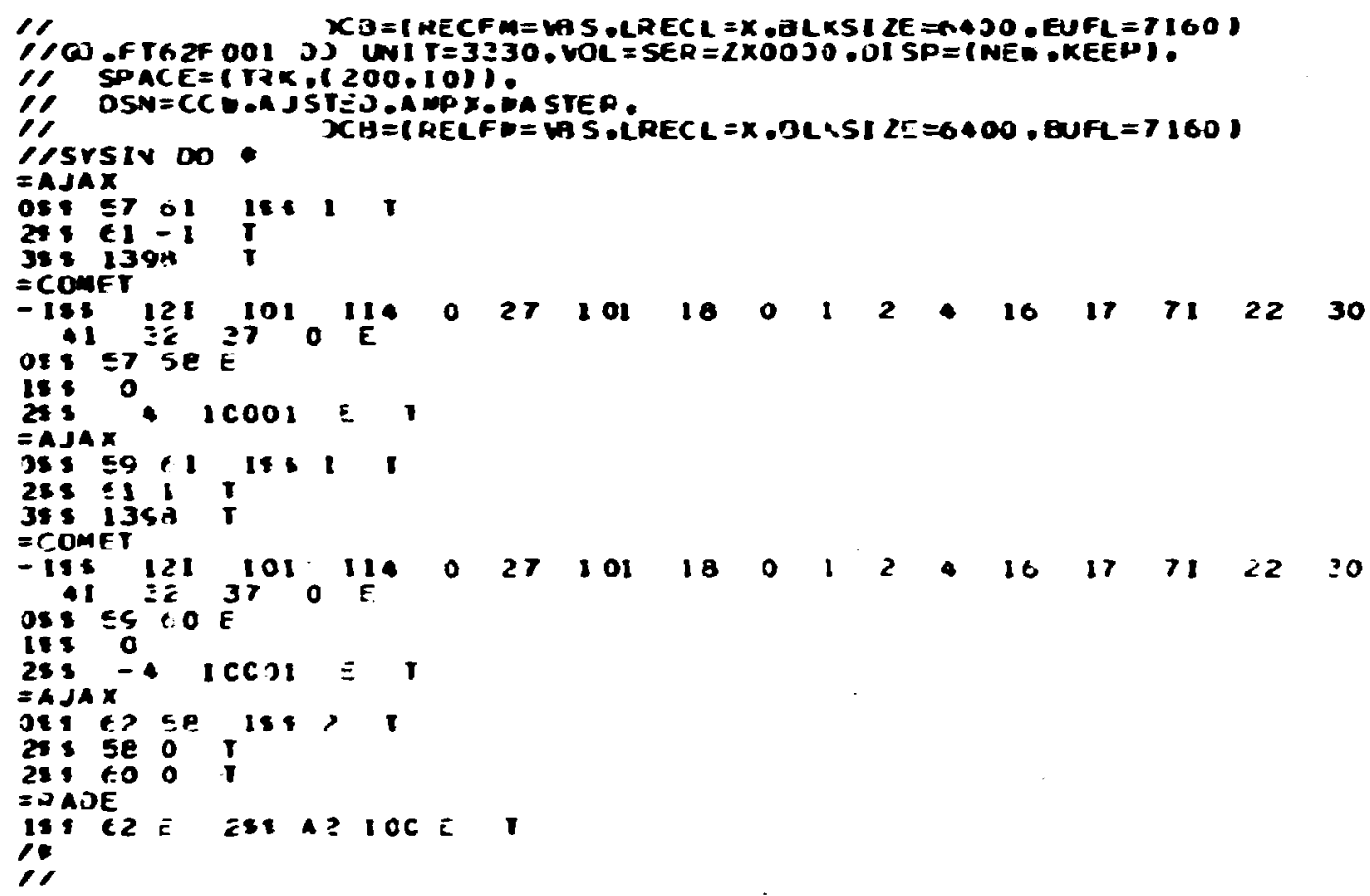




\section{XIV-13}

\section{APPENDIX A}

Fission Spectrum Parameters

The values of the fission spectrum parameters $a$ and $b$ at I MEV are listed for several materials. When $b$ is zero $a$ should be interpreted as a for a maxwellian spectrum. Otherwise the parame'ers are used in the Jefinition of a Watt spectrum.

Material

$a(e v)$

$\underline{b} i \mathrm{eV}^{-1}$

$235 \mathrm{v}$

$238^{U}$

9.88

$E+5$

$2.249 \quad E-6$

$239 \mathrm{Pu}$

8.95064 E+5

$3.2953 \mathrm{E}-6$

$240 \mathrm{pu}$

$9.66 \quad E+5$

$2.842 \quad E-6$

$241 \mathrm{Pu}$

$1.361 E+6$

0

$212 \mathrm{pu}$

$1.3752 \quad E+6$

0

$1.354 E+6$

0 
APPENDIX B

AJAX -- AMPX Module to Merge, Collect, Assent?e, Re-Order, Join and/or C.opy Selected Data Sets from APX Master Interfaces

N. M. Greene

AJAX (Automatic Joining of AMPX $\underline{x}$-Sections) is a nodule to combine data from NPX master interfaces. Options are provided to allow merging from any maber of files in a manner as to allow the user to determine the final muclide ordering. Any form of master interface (neutorn. gamma-ray, neutron-gamma) can be accessed. (In this discussion, interface, file, and library have the same meaning).

AJAX Input Data

Block 1

Os Logical Assignments [2]

1. MMT - Logical number of new library (default = 1)

2. MAX - Logical number of the input file which has the largest Duffer requirements. (AJAX uses MAX and MM in determining total buffer requirements).

1s Number of iiles [1]

1. NFILE - Number of $f i l$ ? requests to be made. (When "reordering" operation: are performed wich require the same file to be accessed several times, each access is counted to determine the value of NFILE).

T Terminate Block 1.

Blocks 2 and 3 are stacked, one after the other, NFILE times.

Block 2

2s File and Option Selection [2]

1. NF - Logical number of file considered

2. $I{ }^{*}=-N$ Delete $N$ nuclides from $N F$ to create the new file on MT

- Sets with duplicate identifiers will not be entered on MI. The first occurrence of an identifier selects that set for the new library. 
XIV-16

$=0$ Add all nuclides to the new file on MAT

$=N$ Add $N$ nuclides from $N$ to create the new file on $M M$

T Terminate Block 2.

Block 3 \{Enter only when $[0 \mathrm{P} T \neq 0$ \}

35 Nuclides Selected [|IOPT|]

Identifiers of nuclides wich are to be added or delr.ted from $\mathrm{NF}$

45 New Identifiers [IIOPTI] (Enter only if an identifier is to be changed.\} This array allows changing the identifier given in the 35 array when it is selected for the new library.

T Terminate Block 3.

9.20.2 AJAX Input/Output Specifications

The following devices are normally needed to execute AJAX:

Logical Number

5

6

15

16

18

19

iAMT(1)

NF
Purpose

Card input

Standard output

Scratch device

Scratch device

Scratch device

Scratch device

Master fiie to be created

File(s) to be merged onto MMT

COMET -- AMPX Module to Correct Selected

Portions of ANPX Master Interfaces

N. M. Greene

COMET (Correct old Master Excessive Iolerances) is a module to correct selected portions of an AMPX master interface. A complete library can be easily corrected. Dperations are provided to ensure that transfer matrices normalize to the proper one-dimensisnal values, or vice versa. Note that. COMET copies the wole library, not just selected master data sets. Although ccupled neutron-gamma libraries can be read in, COMET only "operates" on the neutron data on a master library. 
CORET Ir.put Data Block 1

- IS Special Vector Manipulations [200] (Default = all zeroes) These commands are used when it is desired that certain groupings of cross sections sum to a "total value". For example, it is desirable that $\sigma_{a}=\sigma_{c}+\sigma_{f} c r$ that all inelastic partial values sum to the tctal inelastic values. This array is made up of strings of MT numbers as follows:

Primary MT number--Secondary MT number(s)--Zero

This string directs COMET to ensure that the sin of the cross sections icientified by the secondary $M T$ numbers sums to the cross sections idantified by the primary identifier. Zeroes serve as "strinị delimiters." A positive primary identifier says to sum the secondaries and replace (or form) the primiry values. A negative primary identifier says to nomalize the secondaries such that they sum to the primary. Up to 20 strings can be specified. A negative secondary says to subtract this process so that one can form, for example, $\sigma_{C}=\sigma_{a}-\sigma_{f}$.

OS Logical Assignments [3]

1. MPTO - Old master library (28)

2. MMTN - New master libracy (1)

3. MSC - scratch device (18)

1s Option Trigger [1]

1. IOPT

-1 - vo directly to RECTRY on MMTO and skip any "correcting". This corrects the "Table of Contents". The correct; in is made on MMTO. (MMTN is not ised.).

n - "Correct" the complete master library on Mío and writ.e a new one on MMTN.

$N$ - "Correct" $N$ sets from MMTO and write on MMTN.

25 MT - Numbers of processes to be corrected [100] Place up to $100 \mathrm{MT}$ numbers into the $2 \$$ irray. A negative MT number says to force the 10 cross section to agree with the appropriate iransfer matrix sums while a positive MT 
number forces the transfer matrix to be normalized to the 10 value. Also, enter a signal defined as $10000+M T$ to have the reaction type jesignated by MT located in the ID array placed in the infinite dilution array associated with the Bondarenko factors for the process identified by MT. ihis is crucial in preparing cross section sets there the total cross section has been calculated as ine sur, of adjusted partials. The signal for the total cross section is 10001 .

T Termi rate Birck 1.

Block 2 \{Injut if IOPT >0\}

35 Identifiers for master data sets to be "corrected" [IOPT]

T Terminate Block 2.

\section{COMET Input/Output Assignments}

COMET requires the followins input/output assignments:

\begin{tabular}{|c|c|}
\hline Logical & Purpose \\
\hline 5 & Card input \\
\hline 6 & Standary output \\
\hline 9 & Scratch device (random access) \\
\hline 17 & Scratch device \\
\hline (MTO'28) & Old master library \\
\hline MMTN(1) & Hew master library \\
\hline ics (18) & Scratch Device \\
\hline
\end{tabular}

DIAL--AMPX Module to Produce Selected Edits from AMPX Interfaces

N. M. Greene

DIAL (Display AMPX Libraries) is provided to produce edits from AMPX master interfaces or working interfaces. 


\section{DIAL Input Data}

Block 1

os Logical Assignr.ents [2]

1. MMT - Master interface logical assignment (1)

2. MWT - Horking interface logical assignment (0) Caution -- A single DIAL execution should edit either a master or a working interface, not both. If editing a working interface, MT must be set to zero.

15 Editing Options [4]

1. NEDIT - Number of data sets to be edited (0)

A zero edits a complete interface.

2. NIDN - 0; no effect

1, edit one-dimensional neutron cross sections

3. NIDG - 0 , no effect

1 , edit one-dimensional gamma cross sections

4. NRN - 0 , no effect

1, edit resonance data and Bondarenko factor data

2\$ Transfer Matrices to be Edited [100]

35 Maximum Order of Scattering to be Edited [100]

The $2 \$$ and $3 \$$ arrays select two-dimensional array edits. Place up to $100 \mathrm{MT}$ numbers in the $2 \$$ array to select the desired prociesses. Corresponding entries in the $3 \$$ array specify the maximum order of cross sections to be edited for the different processes. For example, if one desires edits of $P_{s}$ elastic data, and nothing else, the input data would read:

$2 \$ \$ 2$ FO $3 \$ \$$ FO

To edit the total transfer matrix on a working interface, specify

2\$\$ 1 FO 3\$\$ (order of $P_{n}$ ) FO

T Terninate B1.sck 1 .

Block \& (This block is required if NEDIT >0)

45 Identifiers of Data Sets to be Edited [NEDIT] \}NEDIT >0\}

T Terminate Block 2. 


\section{DIAL Input/Output Assignments}

Dial requires the following input/output assignments:

Logical Number

5

6

$\operatorname{MaT}(1)$

$\operatorname{MWT}(0)$
Purpose

Card input

Standard output

Master interface

Working interface

RADE--AMPX Module to Check AMPX Master Cross Section Libraries

RADE (Rancid AMPX Data Exhorcist) is provided to check the multigroup libraries produced by the various AMPX modules. It will check neutron, gamma, or coupled neutron-gamma libraries.

In its present version, the check includes:

1. $\sigma_{T} \stackrel{?}{=} \sigma_{a}+\sigma_{s}$

2. $\sigma_{\text {in }} \stackrel{?}{=}$ s. $\sigma_{n}^{\text {partial }}$

3. $\sigma_{\mathrm{a}} \stackrel{?}{=} \sigma_{\mathrm{c}}+\sigma_{\mathrm{f}}$

4. $\sigma_{c} \stackrel{?}{=} \sigma_{n \gamma}+\sigma_{n}+\sigma_{n p}+\sigma_{n d}+\ldots$

5. $\sigma_{e \ell} \stackrel{?}{=} \sum_{g^{\prime}} \sigma_{e \ell, 0}\left(g+g^{\prime}\right)$

6. ${ }^{\circ} 0\left(g+g^{\prime}\right) ?_{0}$

7. $\sigma_{T}, \sigma_{a}, \sigma_{f}, \sigma_{m y}, \sigma_{n p}, \ldots . \xi_{0}$

8. $-1 \leq \bar{u}\left(g+g^{\prime}\right)=\frac{\sigma_{1}\left(g+g^{\prime}\right)}{3 \sigma_{0}\left(g+g^{\prime}\right)} \leq 1$

In addition to these checks, the code will compute an estimate of the capture binding energy for each neutron group in a coupled neutron-ganma set. on option, one can request a display of differential cross sections. 
RADE Input Dala

Block 1

$1 \$$ Checking Commands [4]

1. MMT - Check the AMPX master Interface on logical MT. This can be a neutron, gama, or coupled neutron-gamma library.

2. MT - Check the AMPX Working/Weightéd Interface on logical MTT. This operation is not operable in the present version.

3. MAN - Check the ANISN binary formatted library on logical MAN.

4. IFM - -1, ANISN library is binary formatted;

$0 \quad 0$, ANISN library is BCD free form;

1, ANISN library is BCD fixed form.

25 Options [20]

1. IOPT1 - number of angles at wich a display of differential cross sections is desired. These anğles will be equally spaced in the cosine range, -1 to +1 . These edits are for the group integrated cross sections and not for each group-to-group transfer.

2. IOPT2 - The $\varepsilon$, in the 1,1000's of a percent, to wich checks are made; e.g., IOPT2 $=1$ is equivalent to $0.001 \%$ checking. This is the default value when IOPT2 is not input or when a zero value is input.

3. IOPT3

- Future checking options; skip or input zeroes.

20. IOPT20

35 ANISN Options [7] MAN $\neq 0$

1. NSET - number of ANISN sets to check.

2. IHT - position of $\sigma_{T}$.

3. IHS - position of $\sigma_{g g^{*}}$

4. ITL - table length.

5. NL - maximum order of scattering.

6. IGM - number of neutron groups.

7. IPM - number of photon groups. 
T Terminate this block.

Block 2 [Input: only when MAN > 0\}

45. Identification Numbers of $P_{0}$ Sets on ANISN Binary Library on Logical MAN [NSET]

$5 \$$ Order of Scattering for Sets of ANISH Data on Logical MAN [NSET]

7* Neutron Group Structure, high-to-low in eV [IGH+1]

8* Ganma Group Structure, high-to-low in eV [IPN+1]

T Terminate this block.

RADE Input/Output Specifications

RADE requires the following input/output assignments:

Logical

5

6

18

19

MMT

MWT

MAN
Purpose

Card Input

Standard Output

Scratch Device

Scratch Device

Master Library

Horking Library

ANISN Library 


\section{$X Y-3$}

\section{THE MUTCRACKER MODULE}

The module huTCRACKER so?ves the so-called "inverse problem" of reactor sensitivity theory. Specifically, this protlem is as follows. Assume the ..ollowing are $g$ ven: (1) a specified reactor system with known sensitivity coefficients, (2) specific perfornance parameter accuracy requirements (e.g., $\frac{1}{2} k$ in $k$, etc.), (3) a cross section. correlation matrix, and (4) a cost functional which gives the cost of an experimental program to measure the required cross sections to within an accuracy specified by the variables $x_{1}, x_{2}, \ldots x_{n}$ (these represent the rms standard deviations of the individuat group cross sections). Then, what are the optimum set of standard deviations on the experimentally measured closs sections which will give a minimum cost to the experimental program and at the same time satisfy the accuracy requirements of the design performance parameters?

Let us represent the variable standard deviations of the cross scctions by the vector $\underline{x}$ and the cost function by $c(\underline{x})$. Let 0 be the correlation matrix and $\underline{s}^{r}$ the sensitivity coefficient vector of performance parametor $r$. Then we minimize $c(\underline{x})$ subject to the constraints:

$$
\begin{aligned}
S^{r} \times D \times S^{r} & \leqslant v_{r} \\
x_{i} & \geqslant 0
\end{aligned}
$$

where $\sim$ indicates the transposed matrix, $X$ is a diagonal matrix with diagonal elements $x_{1}, x_{2}, \ldots x_{n}$, and $v_{r}$ is the maximum value allowed by the constraint on the $r^{\text {th }}$ performance parameter variance. 
This is a nonlinear programing problen with nonlinear (quadratic) inequality constraints. The module MUTCRACKER uses a modification ExCOST of an existing cocie ${ }^{1}$ for noniinear optimization subject to nonl ineär constraints. This subroutine ses a gradient search procedure, with refinements for recognizing patterns in the response surfaces and boundaries on the variables.

The following is a brief survey of the subroutines used by MUTCRACKER. EXCOST finds the optimum $x$ 's subject to the constraints which depend on the input correlations and sensitivities. MIN is a quadratic optimization subjeci to one linear constraint and inequality constraints corresponding to a positive weight vector with a sum of unity. (This routine is not required for the present problems which have but a single weight and a single correlation matrix.) EVAL is a subroutine which returns to the calling routine EXCOST the cost of attaining the accuracy specified by vector $x$. Other routines, in addition to the FIDO generalized input outines, are as follows: Routine ILYICH apportinns lengths of the reauired storage arrays. It calls DATAIN for input, sets some initial convergence and tolerance criteria, calls LIADOV, then calls DATOUT which outputs the results. LIADOV initializes the data required by the two optimization routines EXCOST and VMIN and controls the loop which alternate the above two optimization routines. SPUFFT searches through the CN:'CRX file, XNITIL sets prel iminary initial values of the relative standard deviations $x$ for entry into EXCOST, SCALAR calculates inner or dot products of two vectors, and AMATQ4 is a matrix-inversion routine. MAKEM forms the quadratic required by VMIN; MAKEQ, STOREQ, and GETQ form, store, and retricve the matrices $Q^{r}$ which form the inequality constraints for EXCOST $\left(\tilde{x} Q r x<v_{r}\right)$, and GETS retrieves the sensitivity coefficients as needed. 
$X Y-5$

REFERENCE

1. Kenneth E. Cross, "A Gradient Projection Method for Constrained Optimization," Report K-1746 (May 30, 1968), Uni n Carbide CorporaLion Nuclear Division Computing Technology Center, Oak Ridge, Tennessee. 


\section{NUTCRACKER INPUT \\ $\because$ \\ Integer Parameters [i2]}

1. NP

The number of variable cross section standard deviations.

2. $\mathbf{N}$

The total number of cross section standard deviations = variab!e ones plus fixed ones (for present problems $N P=N=N G)$.

3. METH The number of correlation matrices (METH=1 for present problems).

4. INEQ The number of perfomance parameters wich heve given accuracy requirements with corresponding inequality constraints.

5. HS Lnit number of SENPRO file, if NS=0 the sensitivity coefficients must appear in the card input stream on the $6^{\star *}$ card.

6. IDI An instruction for obtaining the correlaticr. matrix (matrices). If $\mathrm{IDI}=0$ the off-diagonal triangular elements are given in the input card data stream. If $I D I=-1$ the correlation matrix (and some other information) will be found on a COVERX file residing on a unit with reference number IDDI.

7. I001 (see 6. ID1)

8. I02 Set to 0 , reserved for future options.

9. IDD2 Set to 0 , reserved for future options.

10. MINI An instruction determining the form of the cost function: 


$$
\begin{aligned}
\text { MINI }= \pm 1, \pm 2, \pm 3, \pm 4 & \text { If MINI }=1 \quad \text { Cost }=c(x)=\sum_{i} 1 / x_{i} \\
-1 \quad \text { Cost } & =C(x)=\sum_{i}\left(1 / x_{i}\right)^{2} \\
2 \quad \cos t & =c(x)=\sum_{i}\left(c_{i} / x_{i}\right) \\
-2 \quad \cos t & =c(x)=\sum_{i}\left(c_{i} / x_{i}\right)^{2}
\end{aligned}
$$

MIHI $=3,-3,4,-4$ correspond to $1,-1,2,-2$ except -erms for which $x_{i} \leqslant I_{i}$ are not included.

11. IC

Is sequential dcia sit reference number from wich cost coefficients $C_{i}$ will be read in. If $I C=0$ no coefficients are read.. If, however, the correlation matrix was read from a COVERX file, $c_{i}$ 's will already be set equal to the group cross section s'andarc deviations wich were on that file. If $\mathrm{IC}=5$, cost coefficients are in the input card stream.

:2. IXFACT An instruction and scale factor for obtaining initial preliminary values of the vector $x$. If IXFACT $>0.0$ then initial values are read in from the $14^{\star \star}$ card and then multiplied by IXFACT. If IXFACT $\leqslant 0.0$ all components of $x$ along the princtple axes are equal, and the value is determined from the constraints. 
$X Y-8$

455

Sens: tivity Coefficient Identifiers on SEMPRO

[5*METH*INEQ]. Oait if $M S=0$ and if $I D I=0$.

1. IASB Assembly identifier

2. IRESP Response identifier

3. MAT Material identifier

4. MI Reaction identifier

5. NTRU Reaction rate ratio identifier

Wote: Complets the five-integer sequence to identify all needed sensitivity coefficients

$T$

$6 * \star$

Sensitivity Coefficients [5*METH*IMEQ]

Omit if IIS $\neq 0$.

8** Correlatjon Matrix Elements $[(N-1) * N / 2]$

Omit if $101 \neq 0$.

\section{$T$}

$10^{\star \star}$

Maximum Allowed Relative Variances [INEQ]

$12^{\star \star}$

Cost coefficients $C_{i}$ [HP]

Omit if IC $=0$.

16**1 Fixed, nonvariable stindard deviations

$$
x_{i}(i=N P+1, N),[N-N P] \text { omit if } N=N P
$$

$T$

Initial values of $x_{i}(i=1, N P)$ [NP] Omit if IXFACT $<0$ 